\title{
SCALE MODEL SHAKE TABLE TESTING OF SHALLOW EMBEDDED FOUNDATIONS IN SOFT CLAY
}

\author{
A Thesis \\ Presented to the Faculty of \\ California Polytechnic State University \\ San Luis Obispo \\ In Partial Satisfaction \\ of the Requirements for the Degree of \\ Master of Science in Civil and Environmental Engineering
}

By

Steven Kuo

May 2012 
(C) 2012

Steven Kuo

ALL RIGHTS RESERVED 


\section{COMMITTEE MEMBERSHIP}

Scale Model Shake Table Testing of Shallow Embedded Foundations in Soft Clay
AUTHOR:
Steven Kuo
DATE SUBMITTED:
May 2012
COMMITTEE CHAIR:
Robb Moss, Associate Professor
COMMITTEE MEMBER: $\quad$ Gregg Fiegel, Professor
COMMITTEE MEMBER: $\quad$ Bing Qu, Assistant Professor 


\begin{abstract}
Scale Model Shake Table Testing of Shallow Embedded Foundations in Soft Clay
\end{abstract}

\title{
Steven Kuo
}

This research involves shake table testing of $1 \mathrm{~g}$ scale models that mimic the coupled seismic response of a structure on a shallow mat foundation and foundation soil (known as soil-foundation-structural-interaction or SFSI). In previous research, SFSI effects have been quantified through analytical models, numerical analyses, and limited field data. This research works towards increasing the amount of empirical data through scale model shake table testing. A suite of earthquake time histories is considered in evaluating a nominal $10^{\text {th }}$ scale soil-structure model using a flexible wall barrel on a 1-D shake table. San Francisco Young Bay Mud (YBM) is used as the prototype soil and long period narrow building as the prototype structure. Foundation embedment depth, fundamental mode of the structure, and seismic loading function are varied to generate a large database of SFSI results under controlled conditions. The foundation level response is compared to free-field responses to determine the magnitude of the SFSI.

The results confirm the effects of foundation embedment on the peak ground motion and the spectral acceleration at the predominant period of the structure. The foundation level accelerations are deamplified compared to free-field results. Results also confirm the legitimacy of the testing platform and program by comparing the data to previous experimental study. 


\section{ACKNOWLEDGEMENTS}

I am truly humbled and inspired through this arduous journey. I would like to express my deepest gratitude to everyone who has helped me throughout my undergrad and graduate studies. I want to thank:

My parents and siblings for always being there for me and giving me the support to grow and mature into who I am today.

Dr. Moss for being my mentor since 2007 and challenging me intellectually during my research. You have shaped my career at Cal Poly and my future as an engineer.

Dr. Qu and Dr. Fiegel for serving as my committee member, providing constructive feedbacks regarding my thesis, and helping me through my research hurdles.

Vic Crosariol for paving the way for the shake table testing program at Cal Poly, so I was able to continue on the foundation he built.

My fellow researcher and grad students, Ron Noche and Justin Martos, for hauling clay, moving equipment, and testing for countless hours with me.

Grad room buddies and classmates, Matt Anderson, Kira Ortiz, Rachel Goossens, Taki Chrysovergis, Gary Guo, Collin Leung, and Jacky Ng, for sharing those tough school moments, interesting conversations, and words of encouragement.

Lastly, I'd like to thank my girlfriend Stephanie Liu. She has been my personal motivator on my side day in and day out. Without her encouragement and help, it might have taken me a few more years before I can finish.

It has been a challenging and rewarding process, and I am excited to face the future knowing that I have people I can count on and a solid education. Thank You. 


\section{Table of Contents}

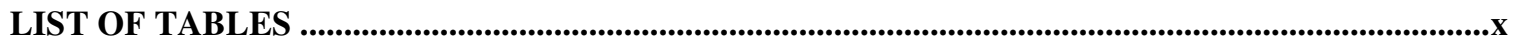

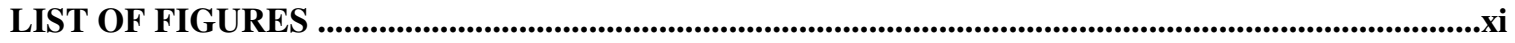

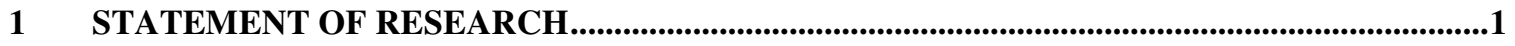

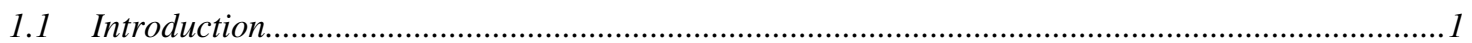

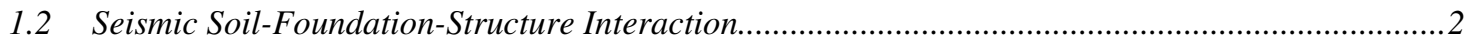

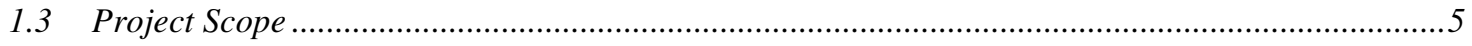

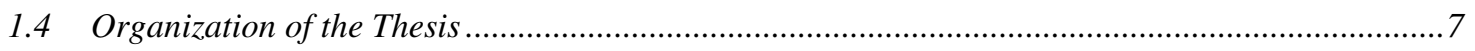

2 LITERATURE REVIEW ...................................................................................................

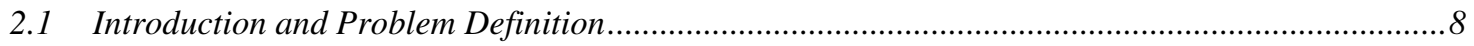

2.1.1 Components of the Soil-Foundation-Structure Interaction ....................................................10

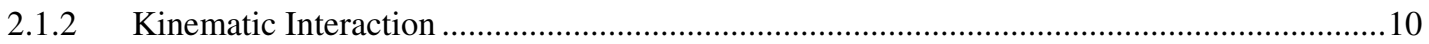

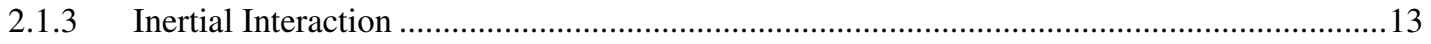

2.2 Methodologies for Soil-Structure Interaction Analysis...............................................................15

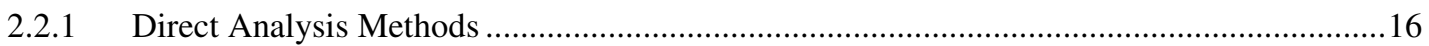

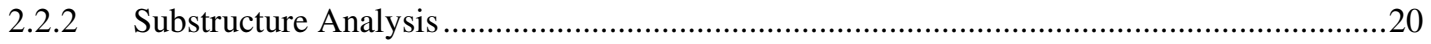

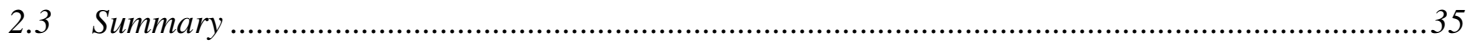

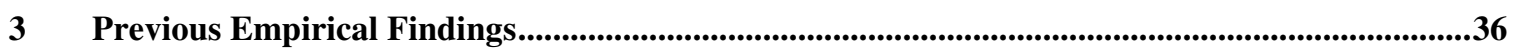

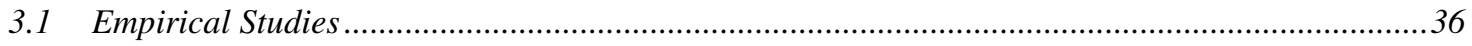

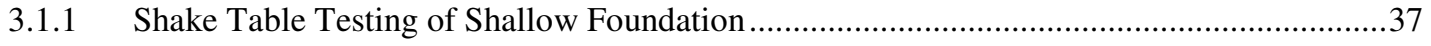

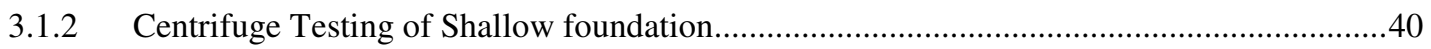

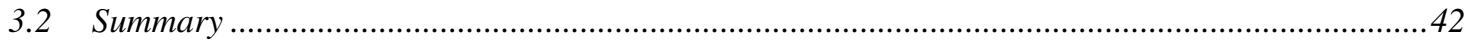

4 DEVELOPMENT OF TESTING PLATFORM ................................................................................44

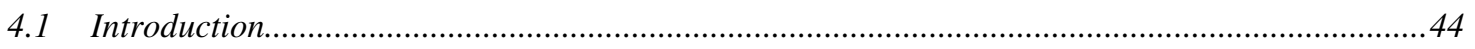

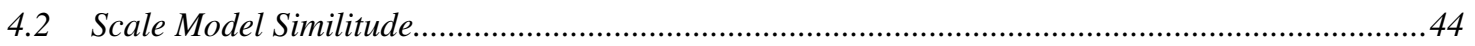

4.2.1 Scale Modeling Similitude for Shake Table Testing ............................................................4

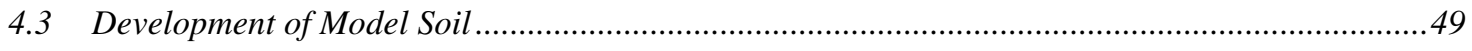

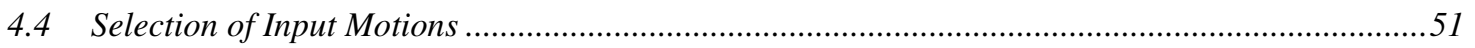




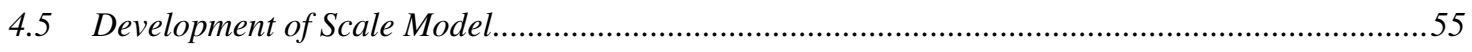

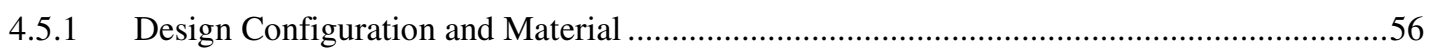

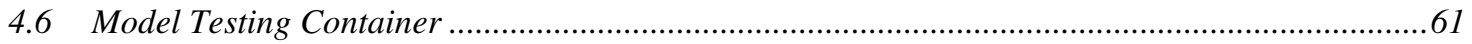

5 EXPERIMENTAL TESTING PROGRAM ...................................................................63

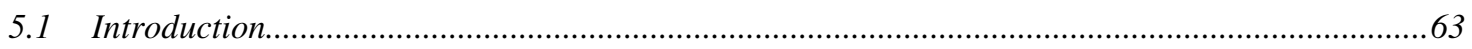

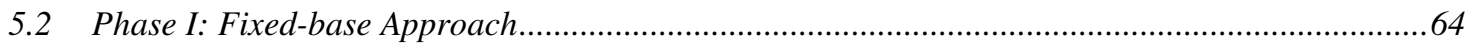

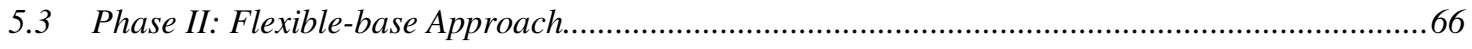

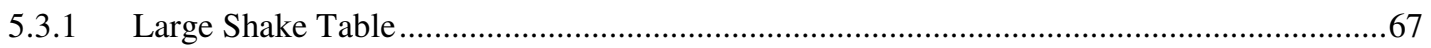

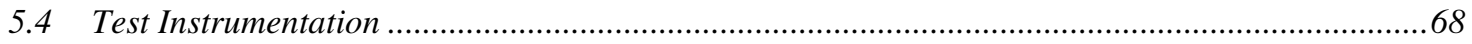

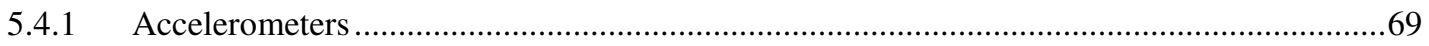

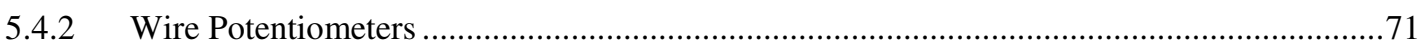

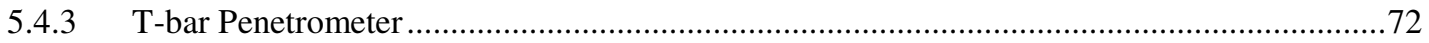

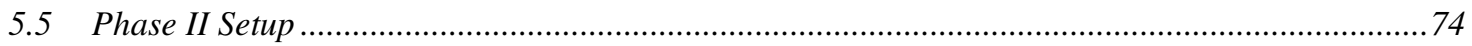

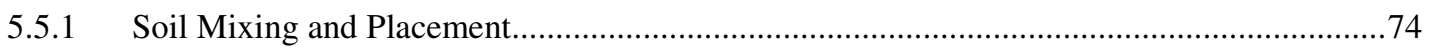

5.5.2 Placement and Embedment of Scale Model ..........................................................75

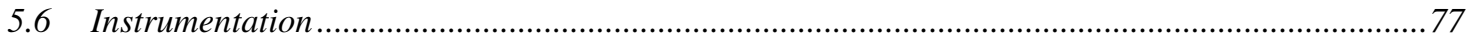

5.6.1 Model instrumentation and Configuration................................................................. 77

5.6.2 Testing Container instrumentation and Configuration .................................................79

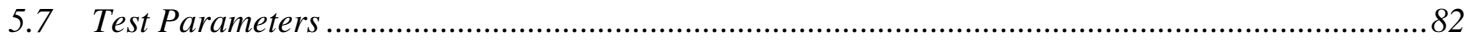

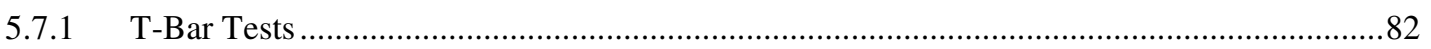

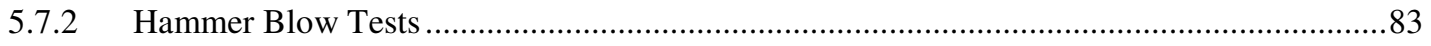

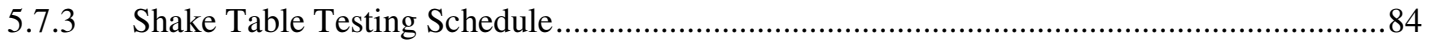

$6 \quad$ EXPERIMENTAL TESTING RESULTS ..............................................................................89

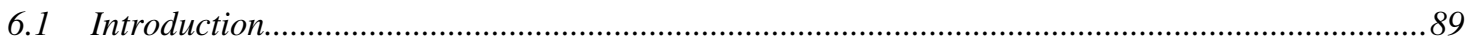

6.2 Phase I Results: Fixed Based Performance .....................................................................89

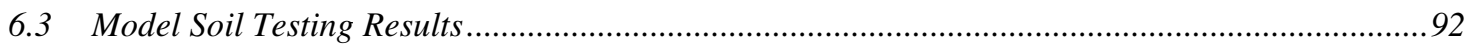




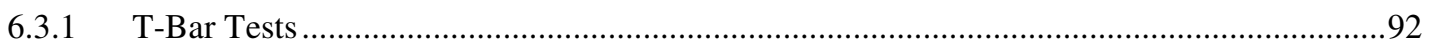

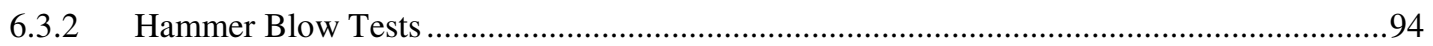

6.3.3 Development of Shear Wave Velocity Profile ............................................................97

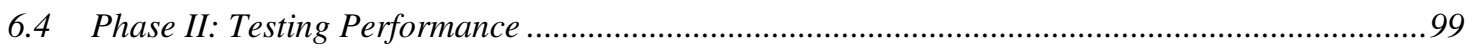

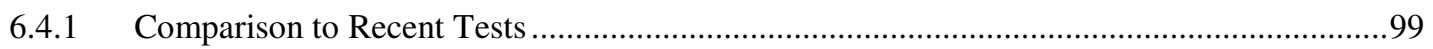

6.4.2 Displacement Comparison............................................................................... 102

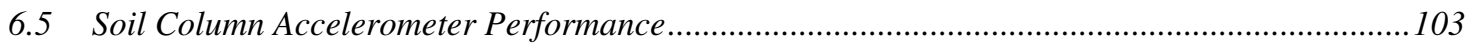

6.5.1 Free-field Array vs. Model Array ............................................................................ 104

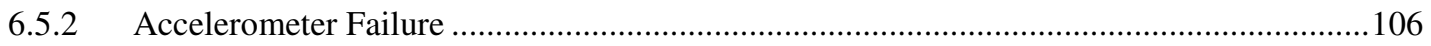

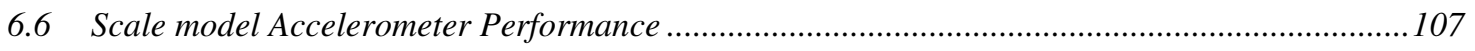

6.6.1 Foundation Horizontal Recording ................................................................. 107

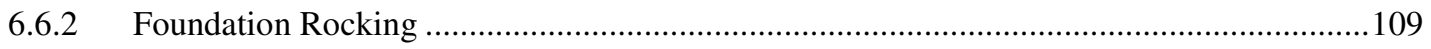

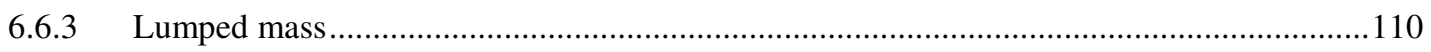

6.7 Peak Acceleration Results and Comparison ........................................................................111

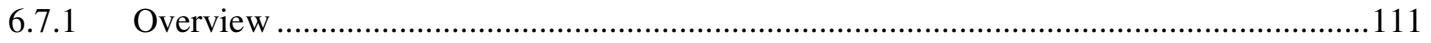

6.7.2 Data Comparison to Previous Research .................................................................... 112

6.7.3 Effects of Embedment ....................................................................................... 114

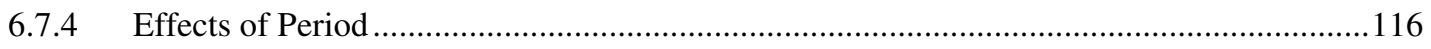

6.8 Spectral Acceleration Results and Comparison .............................................................. 118

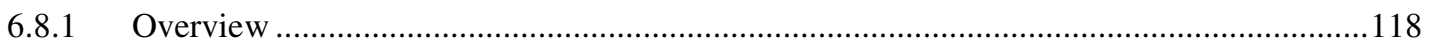

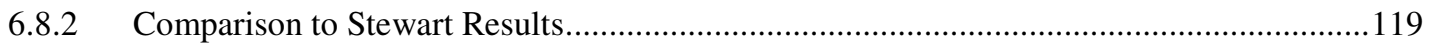

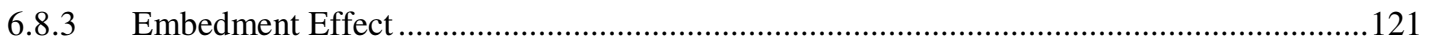

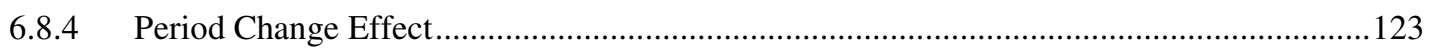

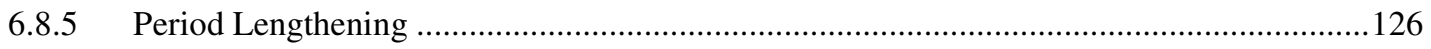

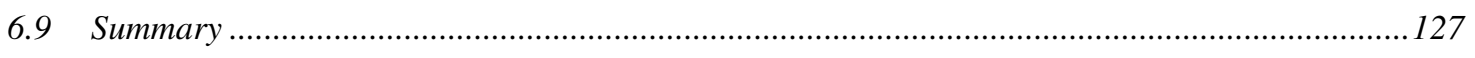

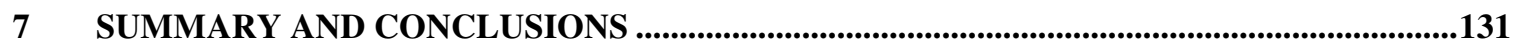

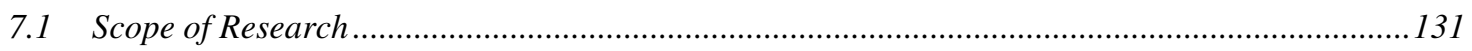




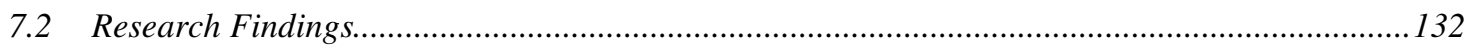

7.3 Recommendations for Improvement and Future Research ….................................................134

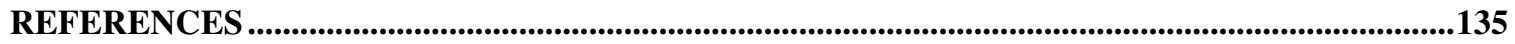

APPENDIX A - DISPLACEMENT DATA FROM THE WIRE POTS ..................................................141

APPENDIX B - SPECCTRAL ACCELERATION RESULT GRAPHS .........................................148 


\section{LIST OF TABLES}

Table 3-1 The period lengthening ratio and the foundation damping ratio of the four models tested from the shake table testing (from Hosseinzadeh and Nateghi 2003)

Table 4-1 SFSI interaction modes and relevant variables (adopted from Meymand, 1998; Crosariol, 2010)

Table 4-2 Scale factors for pertinent engineering variables in terms of geometric scaling factors $(\lambda)$ (adapted from Iai, 1989; Meymand, 1998; Crosariol, 2010)

Table 4-3 Prototype soil properties and the model soil mix for this study (after

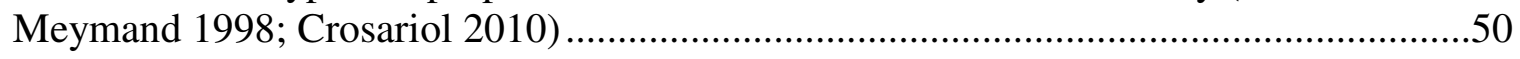

Table 4-4 List of motions used for this study and earthquake information ..................... 51

Table 5-1 Phase II instrumentation listing and nomenclature (after Crosariol 2010)....... 82

Table 5-2 T-bar pull out test for Phase II testing ....................................................... 83

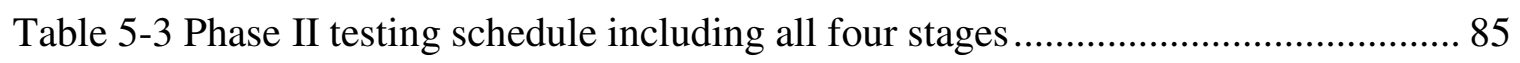

Table 6-1 Final setups for the SDOF lollipop of the two target prototypes ................... 91

Table 6-2 Summary of hammer blow testing for both arrays including the test ID,

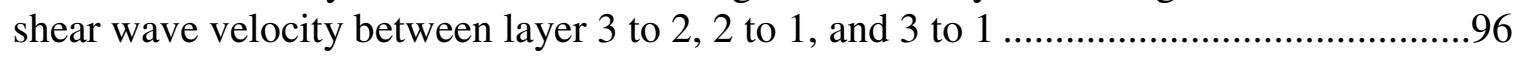

Table 6-3 Summary of percentage increase in spectral amplitude at the predominant period at the model array compared to the free-field array ....................................... 106

Table 6-4 Average percentage increase of peak spectral value from 3F to $3 \mathrm{M}$ between each testing stage

Table 6-5 Average percent reduction in spectral acceleration at $\square$ of the foundation level when compared to free-field level.............................................................119

Table 6-6 Average period lengthening ratio from this study .................................... 127

Table 6-7 Summary of peak acceleration data........................................................ 130

Table 6-8 Summery of spectral acceleration at $\square$ data.......................................... 130 


\section{LIST OF FIGURES}

Figure 1-1 - Schematic of modes of simple seismic response for a SFSI (after Meymand 1998)

Figure 1-2 - Comparison of free-field and foundation level structure motions: (a) peak acceleration data; (b) 5\% damped spectral acceleration comparison (from Stewart et al., 1999b)

Figure 2-1 Examples of collapse of two structures in San Francisco's Marina District due to "soft" ground floor during the 1989 Loma Prieta Earthquake (from Seed et al. 1990)

Figure 2-2 More examples of structural damage in the San Francisco's Marina District with "soft" ground floor due to the 1989 Loma Prieta Earthquake (from Seed et al. 1990)

Figure 2-3 Cut out FE mesh of the model in Taiwan (From Borja et al., 1999)

Figure 2-4 The full 3-d model of the OpenSees FEM analysis (From Preisig and Jeremic 2005)

Figure 2-5 The four cases of FE models used for comparative study of SSI effects on liquefiable soils: shallow foundation, monopole foundation, deep pile foundation, and free-field (from Buehler 2006).

Figure 2-6 Numerical grid and model component in FLAC3D (from Rayhani and Naggar 2008)

Figure 2-7 Substructure method to analysis of the SFSI by breaking down into three steps (from Stewart et al. 1998).

Figure 2-8 Simplified model for inertial interaction analysis (from Stewart et al. 1999a)

Figure 2-9 Foundation stiffness and damping factors for elastic and viscoelastic halfspaces, $v=0.6$ (after Veletsos and Verbic (1973) and Stewart et al. (1998))

Figure 2-10 Schematic plan of experimental site (from Lee and Jennings 1984)

Figure 2-11 Embedded soil-foundation-structure system on finite soil layer for Elsabee and Morray's analysis (after Elsabee and Morray (1977) and Stewart et al. (1998))

Figure 2-12 Comparison plots of period lengthening ratios and foundation damping factors for single degree-of-freedom structure with surface and embedded foundations $(v=0.45 ; \beta=5 \% ; \gamma=0.15 ; \zeta=5 \%)$ [after Veletsos and Nair (1975); Bielak (1975); Aviles and Perez-Rocha (1996); Stewart et al. (1998)]

Figure 2-13 BNWF model schematic (from Gajan et al. 2008) 
Figure 2-14 Concept of macro-element contact interface model, and the associated forces and displacements at foundation-soil interface during combined loading (from Gajan and Kutter 2009).

Figure 3-1 Picture of the shake table experiment set-up with a detailed picture of the model foundation located on sand deposit surface (from Paolucci et al, 2008) .38

Figure 3-2 Setup of the shake table testing (from Hosseinzadeh and Nateghi 2003).......40

Figure 3-3 Setup of the centrifuge model and the placement of accelerometers and LVDTs (from Rayhani and Naggar 2008)

Figure 3-4 Setup and instrumentation of the centrifuge in the experiment (from Pitilakis and Clouteau 2010)

Figure 4-1 Spectral acceleration summary of the 14 motions used in this study. These motions cover a range of various peak spectral accelerations and corresponding periods.

Figure 4-2 Scaled Joshua Tree motions for both horizontal azimuths of the 1992 Landers earthquake with compressed time step by $\lambda^{0.5}$

Figure 4-3 Scaled El Centro motions for both horizontal azimuths of the 1940 Imperial Valley earthquake with compressed time step by $\lambda^{0.5}$

Figure 4-4 Scaled TCU075 motions for both horizontal azimuths of the 1999 Chi Chi earthquake with compressed time step by $\lambda^{0.5}$

Figure 4-5 Scaled Supersition Mountain motions for both horizontal azimuths of the 1979 Imperial Valley earthquake with compressed time step by $\lambda^{0.5}$

Figure 4-6 Scaled Los Gatos motions for both horizontal azimuths of the 1989 Loma Prieta earthquake with compressed time step by $\lambda^{0.5}$

Figure 4-7 Scaled Lake Hughes motions for both horizontal azimuths of the 1994 Northridge earthquake with compressed time step by $\lambda^{0.5}$

Figure 4-8 Scaled Cape Mondecino motions for both horizontal azimuths of the 1992 Cape Mondecino earthquake with compressed time step by $\lambda^{0.5}$

Figure 4-9 An angle snapshot of the final scale model. The model is secured to the small shaking table, which is introduced in the next chapter. Note the accelerometer attached to the weight plate for vibration data collection

Figure 4-10 Scale model design and materials used.

Figure 4-11 Different model soil containers for SSI shake table testing (after Meymand, 1998) 
Figure 4-12 Dynamic analysis of different model soil containers showing that the flexible wall barrel provides similar response when compared to prototype field conditions (after Meymand, 1998).

Figure 4-13 The testing platform shown consists of the shake table, the flexible wall barrel, and the associated elements. Located at Cal Poly's Parsons Earthquake Lab .....

Figure 5-5-1 Small shake table used in the Phase I study. Input motions were controlled by Dactron shaker controller program from a nearby computer

Figure 5-2 An accelerometer was attached to the side of the weight plates to collect acceleration data during the sine sweep test.....

Figure 5-3 Snapshot of Parson's Earthquake and Geotechnical Engineering Laboratory at Cal Poly....

Figure 5-4 A close up view of the SCXI 1001 chassis that receives voltage signals through the instrumentation cables and converts to appropriate measuring values......

Figure 5-5 Accelerometer 353B52 used to measure the shake table output acceleration

Figure 5-6 Accelerometers 393B04 with the mini-foundation housing and silicon sealant protection .70

Figure 5-7 Accelerometer J353B51 used as a foundation rocking monitor .71

Figure 5-8 The top wire pot is connecting to a hook on the surface of the rubber at the same level as the surface of the soil column to measure displacement during testing .......72

Figure 5-9 T-bar testing device with (a) $2.2 \mathrm{kN}$ load cell threaded to T-bar rod and eye bolt for pulling using the crane and (b) T-bar penetrometer steel rod that is embedded in the soil ( From Crosariol 2010) .74

Figure 5-10 Soil water content in the testing container .75

Figure 5-11 Scale model with the foundation resting on the model soil surface in the testing container.

Figure 5-12 Scale model embedment process with (a) excavation of a cavity in the soil column and (b) lowering the model in place using overhead crane.

Figure 5-13 Plan view of the scale model with accelerometers placement and dimension.

Figure 5-14 Accelerometers mounted on the foundation to (a) measure acceleration in the shaking direction and twisting (b) measure vertical acceleration for rocking; the level was used to check the foundation overall level during embedment.

Figure 5-15 Plan view of the testing container with the scale model embedded and surface accelerometers in place 
Figure 5-16 Plan and elevation view of the accelerometer placement in the testing container.

Figure 5-17 Two T-bars being held in place using a wooden beam and two clamps. Once the container is filled, the beam and clamps were removed

Figure 6-1 Acceleration vs. driving frequency plot of the fixed base small shake table test to determine the first natural mode of vibration of the SDOF model. The model has a fundamental frequency of $2.6 \mathrm{~Hz}$, which also represents a natural period of vibration of $0.38 \mathrm{~s}$ for the model and $1.2 \mathrm{~s}$ for the prototype after similitude analysis .....90

Figure 6-2 Converted prototype period versus the model lumped mass height

Figure 6-3 T-bar undrained shear strength results comparison of the four trials in Phase II.

Figure 6-4 Comparison between the average undrained shear strength from the study within and the Crosariol Phase II soil column (after Crosariol 2010) ....

Figure 6-5 Typical wave forms from a top-down hammer blow test for the bottom soil column. Results from MAE5 is shown here

Figure 6-6 Dickenson (1994) relationship that correlates soil shear strength to shear wave velocity for clay soil

Figure 6-7 Soil shear wave velocity profile interpretation progression: From T-bar profile from undrained shear strength, and scaled shear wave velocity profile using shear wave velocity data from hammer blow test, and the best estimated soil shear wave velocity profile.

Figure 6-8 best estimated shear wave velocity profile of this study

Figure 6-9 5\% damped surface response spectra comparison for the Joshua Tree motions

Figure 6-10 5\% damped surface response spectra comparison for the El Centro motions

Figure 6-11 5\% damped surface response spectra comparison for the TCU Chi Chi motions

Figure 6-12 Comparison of displacement data from the string pots between the soil surface level and the shake table level for the El Centro 180 motion.

Figure 6-13 5\% damped spectral acceleration time history for all the acceleration data from the 3_ELC180_H test

Figure 6-14 5\% damped spectral acceleration time history for the table, level 1, level 2, and level 3 acceleration data from the 1_ELC180_H test 
Figure 6-15 5\% damped spectral acceleration time history for all the acceleration data from the 2_ELC180_H test.

Figure 6-16 Time history comparison for the horizontal accelerometers on the foundation for test 1_LGP000_L

Figure 6-17 5\% damped spectral response on the horizontal accelerometers on the foundation for test 1_LGP000_L.

Figure 6-18 5\% damped spectral response on the vertical accelerometers on the foundation for test 3_LGP000_L

Figure 6-19 Time history comparison for the vertical accelerometers on the foundation for test 3_LGP000_L

Figure 6-20 5\% damped spectral response comparison for the lumped mass accelerometer for motion LGP000.

Figure 6-21 Free-field versus foundation level peak acceleration for all the empirical tests

Figure 6-22 a) Linear trend lines for each testing Stage and the original data points b) Comparison of Stewart's results and the study within using linear trendlines (Stewart 1998)

Figure 6-23 Comparison between no-embedment and full-embedment results of peak acceleration

Figure 6-24 Comparison of peak acceleration results between Model High and Model Low for Stage 1 ......

Figure 6-25 Comparison of peak acceleration results between Model High and Model Low for Stage 2 .

Figure 6-26 Comparison of peak acceleration results between Model High and Model Low for Stage 3

Figure 6-27 Spectral acceleration at the predominant flexible-base period comparison between free-field and foundation level

Figure 6-28 a) Comparison of free-field and foundation level 5\% damped spectral acceleration@ @ ; b) Comparison of Stewart's results and the study within using linear trendlines (Stewart 1998).....

Figure 6-29 Correlation between embedment ratio (e/r) and the average deamplification of spectral acceleration at

Figure 6-30 Comparison of spectral acceleration at $\square$ between Model High and Model Low for Stage 1 
Figure 6-31 Comparison of spectral acceleration at $\square$ between Model High and

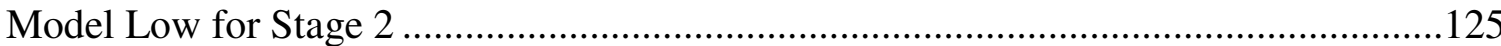

Figure 6-32 Comparison of spectral acceleration at $\square$ between Model High and Model Low for Stage 3 .............................................................................................125 


\section{CHAPTER 1 - STATEMENT OF RESEARCH}

\subsection{Introduction}

Earthquakes occur daily around the world - most of them are too small for human beings to acknowledge; however, a huge magnitude earthquake can have catastrophic effects on society. Buildings, bridges, and important lifelines are at risk for serious damage, and death is an ever-present consequence. It is important for designers to understand the effects of seismic excitation on buildings in order to mitigate potential damage. Past reliable studies have found that the effects of the soil-structure-interaction (SSI) alter the seismic response of shallow foundation buildings (Elsabee and Morray 1977; Seed and Lysmer 1980; Lin and Jennings 1984; Aviles and Perez-Rocha 1996; Gazetas and Mylonakis 1998; Stewart et al. 1999a/b; Kim and Stewart 2003). Yet there are still many issues associated with the effects of the soil on the structural elements during seismic loading that can be better explored.

The relationship between the SSI effects and the foundation embedment depth has become significant in earthquake engineering design. It was specifically explored for the analysis of nuclear reactor structures starting in the 1970's (Seed and Lysmer 1980). Historically, the SSI effects have been neglected for conventional structural design of light structures in reasonably stiff soil; however, the effects of SSI are considerable for heavy structures located in relatively soft soils (Wolf 1985; Rayhani and Naggar 2008). Furthermore, the SSI effects have been traditionally considered as beneficial to the structural system under seismic loading as the lateral fundamental period lengthens and overall system damping increases, thus reducing the overall base-shear demand 
(Mylonakis and Gazetas, 2000). This notion results in simplified provisional seismic codes, including the National Earthquake Hazards Reduction Program (NEHRP) seismic design code (BSSC, 1997) and Applied Technology Council (ATC, 1978) codes that suggest conservative design by ignoring SSI effects (Mylonakis and Gazetas, 2000). The simplified practice is likely fueled by the lack of well-documented field performance data; however, recent empirical data has demonstrated that the SSI influence can in fact be detrimental to the overall building (Stewart et al. 1998). With a limited database for SSI analytical methods calibration, empirical data from controlled lab conditions is needed to provide additional crucial information on SSI effects.

\subsection{Seismic Soil-Foundation-Structure Interaction}

In analyses of structures located on rock, the structures are assumed to be fixedbase. The seismic motion experienced by the structure is the same as the motion experienced by the rock. However, the seismic waves can propagate vertically through soft soil stratum and result in significant amplification of seismic motion at the surface. The seismic motion experienced by the surface of the site without any influence of structural elements is known as free-field motion. When a structure is built on the freefield site, it is subject to influence from the softer soil and no longer has a fixed-base; this condition is known as flexible-base. The presence of the structure interacts with the surrounding soil and results in a further variation of the motion experienced by both the soil and the structure. This coupled relation between the soil and the structure is known as soil-structure interaction (SSI). The SSI issue has been greatly explored in recent years for various civil engineering applications, such as deep foundations, bridge piers, dams, and underground structures design (Wolf 1985). For this research, the primary focus is 
the interactive relationship between soft clay soil and shallow foundations, which is referred to herein as soil-foundation-structure interaction (SFSI).

The complexity of SFSI involves various modes of interactions, which can be simplified schematically in Figure 1.1. The main components of the system include the seismic energy source, far field soil, near field soil, superstructure, and the supporting foundation. The key interactions include kinematic interaction, inertial interaction, radiation damping, coupled lateral responses, construction effects, and axial loads. Further descriptions of each mode are presented below.

- Kinematic interaction is the inability of the foundation to comply with the free-field deformation during seismic loading due to differences in stiffness between the foundation and the surrounding soil. These deviations are mainly a result of base slab averaging, embedment effects, and wave scattering (Elsabee and Morray 1977; Luco and Mita 1987; Veletsos and Prasad 1989).

- Inertial interaction consists of inertia developed by the structure due to its own vibration and dynamic response, which can cause base shear and moments that result in displacement or rocking relative to the free-field (Stewart and Seed. 1998). In general, the effects of inertial interaction are more pronounced than those of kinematic interaction for SFSI of shallowly embedded foundations (Wolf 1985). The focus of this thesis is primarily on the effects of inertial interaction of shallowly embedded foundations.

- Physical interaction between the soil and foundation can occur before and during seismic loading. Installation and construction apply loading that induces soil 
displacement and strain. Furthermore, dynamic loading from seismic activities can induce additional stress (Meymand 1998). At the interface of the soil and the foundation near the ground surface, gaps can develop as a result of dynamic cyclic loading. For cohesionless soil, gaps can be filled and compacted; however, they can remain open for cohesive soil, thus further reducing the soil-foundation contact surface and lowering the soil-foundation lateral stiffness (Meymand 1998).

- Damping is an important factor for any seismic design, and it includes hysteretic, structural (foundation), and radiation damping. Radiation damping occurs as the wave dissipates through the surrounding soil or other material from the source, resulting in the reduction of specific elastic energy and amplitude. In terms of soil and foundation, the stiffer foundation vibrates and releases more energy into the nearby soil, thus reducing the dynamic response of the foundation.

As illustrated in Fig. 1-1, the dynamic coupling relations between the components are complicated. To accurately capture and analyze the non-linear effects of SFSI, timeconsuming, expensive, and complex numerical analyses are required. More simplified and efficient analytical procedures for SFSI are discussed in Chapter 2. 


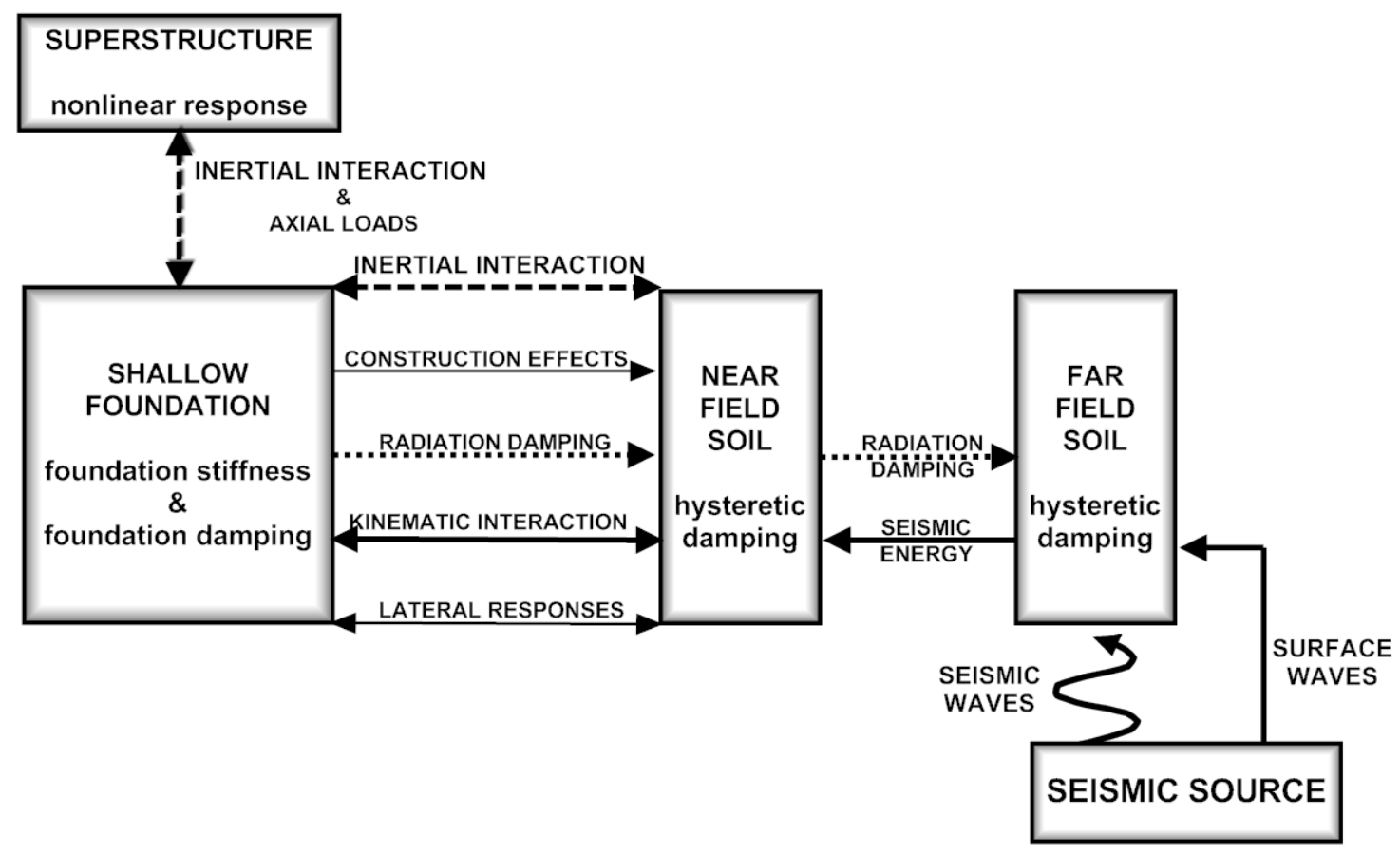

Figure 1-1 - Schematic of modes of simple seismic response for a SFSI (after Meymand 1998)

\subsection{Project Scope}

In past studies, the effects of SFSI were found to alter the seismic response of buildings with embedded foundation (Bielak 1975; Lin and Jennings 1984; Aviles and Perez-Rocha 1998; Stewart et al., 1999a/b; Takewaki et al. 2002; Paolucci et al. 2007). These SFSI effects were quantified through analytical models, numerical analyses, and limited field data. Notably, a study conducted by Stewart et al. (1997) gathered strong motion data from 58 sites with various structures and soil conditions. The results were analyzed to quantify the inertial interaction effects on modal parameters of structures. Data of free-field ground motions and foundation-level ground motions were compared and displayed, as seen in Fig. 1-2. The data shown suggest a reduction trend in peak 
ground accelerations and spectral accelerations at the foundation-level recording when compared to the free-field data.

Other issues associated with the effects of soil on foundation-structural elements during seismic loading can be further explored. The majority of past SFSI studies and empirical experiments have been conducted using liquefiable cohesionless soils. However, seismic activities often affect structures located on soft cohesive soils as well, thus creating a need to augment the limited empirical database for soft cohesive soils with respect to SFSI effects. The primary goal of this research is to provide conclusive empirical data through scale model testing in a controlled shake table lab to supplement the meager empirical data for SFSI effects on soft clay soils. Therefore, this research works to increase the amount of limited empirical data through scale model shake table testing of soft clay soils by investigating the effects of various foundation embedment depths in relation to the structural response.

For this research, a testing platform, originally developed by Meymand (1999) at U.C. Berkeley and further modified and validated by Crosariol (2010) at Cal Poly, was used. This one-directional shake table allows for investigation of SFSI effects in controlled and systematically varied conditions. A scale shallow foundation structure is subject to varying embedment depths in the model soft clay and then tested with a suite of one-directional strong-ground motions. Acceleration, displacement, and pressure data are collected from the soil and foundation to quantify the SFSI effects. The empirical data are analyzed using substructure methods presented in Chapter 2 to further confirm the effects of embedment of foundation on the structural modal responses 

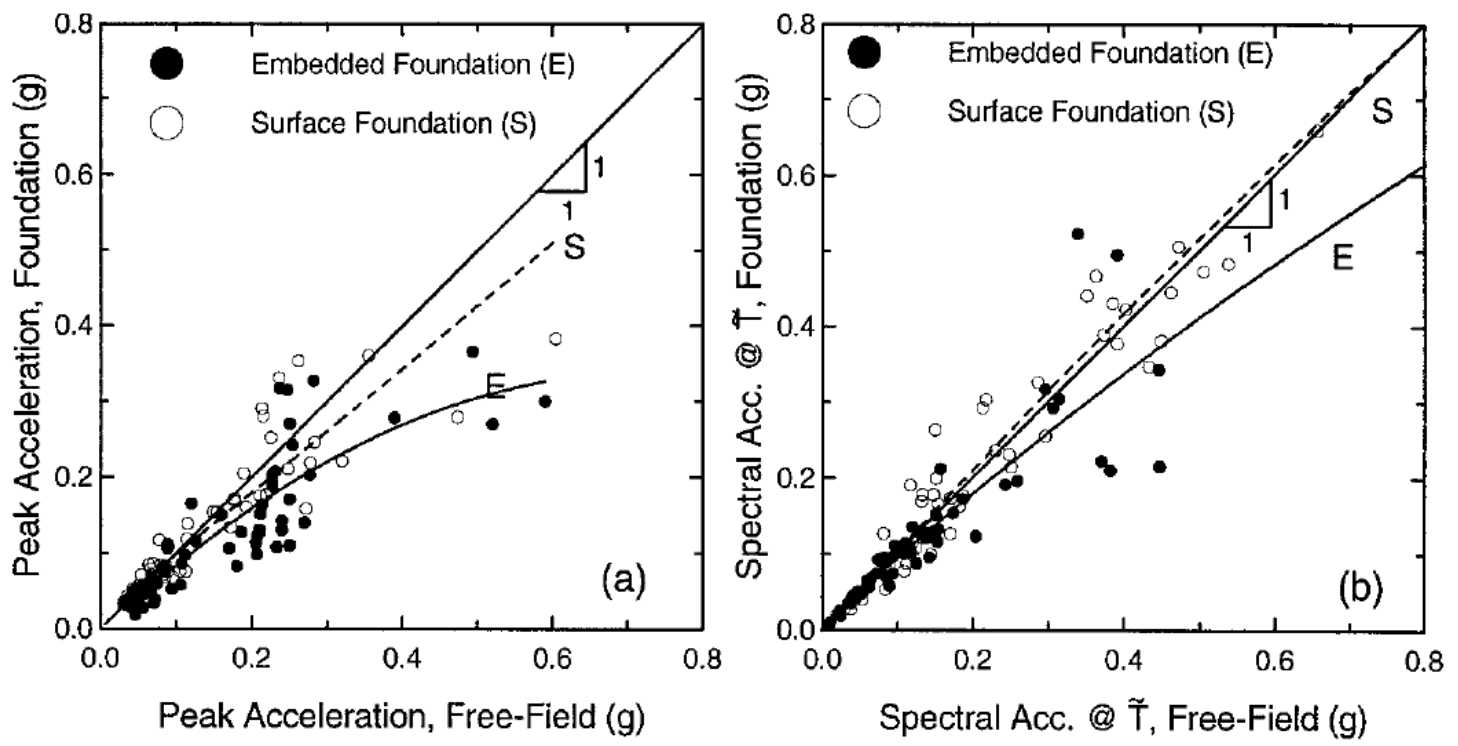

Figure 1-2 - Comparison of free-field and foundation level structure motions: (a) peak acceleration data; (b) 5\% damped spectral acceleration comparison (from Stewart et al., 1999b)

\subsection{Organization of the Thesis}

Chapter 2 includes theories behind soil-structure-interaction, primarily focusing on inertial interaction, including period lengthening and foundation damping. Chapter 3 provides a summary of past numerical and experimental analyses on SFSI. Chapter 4 gives an overview of the testing platform and the scale model development, including model soil, testing container, and scale model scaling relation. Chapter 5 details the testing setup on the instrumentation placements, various testing procedures pertinent to this study, and testing schedules. Chapter 6 provides quantitative results and discussion for the physical shake table testing in addition to comparison study against Stewart's results. Finally, research findings, discussion, and recommendation for future study are detailed in Chapter 7. 


\section{CHAPTER 2 - LITERATURE REVIEW}

\subsection{Introduction and Problem Definition}

Building codes generally use the fundamental period of the buildings to evaluate the response to seismic excitation. These empirical formulas provided by the seismic codes typically ignore the soil flexibility and its ability to influence the overall fundamental period of the structure (Khalil et al. 2007). In fact, this conservative oversimplified code base approach is driven by the common notion that the soil-structure interaction is beneficial to the behavior of structures. It lengthens the fundamental period and leads to higher damping of the system during seismic loading, thus reducing the overall seismic demand. However, some recent case studies suggest possible detrimental effects from soil-structure-interaction (SSI), and neglecting this can lead to unconservative design for structures, especially those located on soft soil (Gazetas and Mylonakis 1998; Mylonakis and Gazetas 2000).

Mylonakis and Gazetas (2000) reported that SSI caused an increase in seismicinduced response on structures during the 1985 Mexico earthquake, despite an increase in damping. Several 10- to 12- story buildings founded on soft clay suffered severe damage when their fundamental periods increased from 1.0 second to 2.0 seconds as a result of SSI (Resendiz and Roesset 1987). The observed structural behavior contradicted the outlined expectation from the conventional code base design.

Seed et al. (1990) presented a reconnaissance report on the 1989 Loma Prieta Earthquake in Northern California and identified several causes for the significant 
damage. The majority of the building damage in the Marina District of San Francisco was located on soft cohesive soil. The seismic motion propagated vertically to the ground surface through the soft cohesive soil and resulted in excessive base shear for the structures. This acceleration amplification of long period motions was the primary cause of damage as the failed buildings' fundamental periods resonated with the soil during seismic excitation. Most observed destruction occurred on "weak" ground floor structural elements, primarily garages and thin walls that did not have adequate lateral shear force resistance capacity. Such failures are illustrated in Fig. 2-1 and 2-2. This further warranted the need to include the soft soil flexibility and ground motion amplification in the overall design considerations. Designers need to evaluate the influence of SFSI, especially for soft clay sites to avoid catastrophic damage.

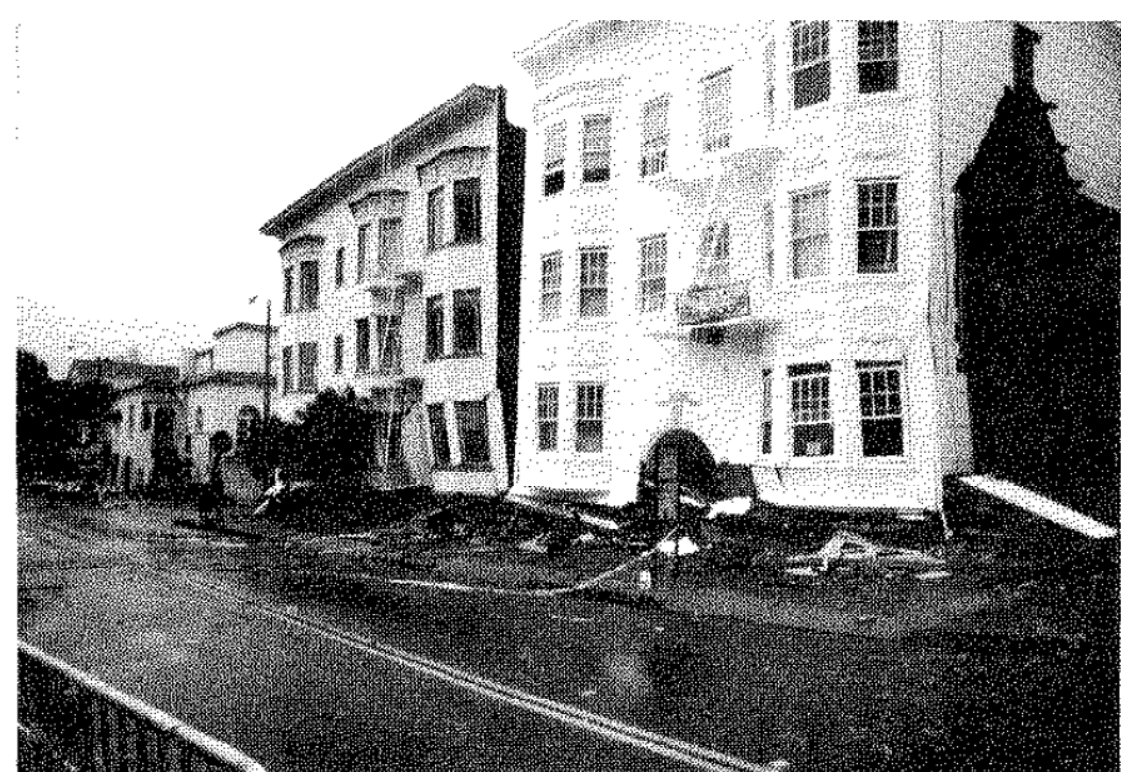

Figure 2-1 Examples of collapse of two structures in San Francisco's Marina District due to "soft" ground floor during the 1989 Loma Prieta Earthquake (from Seed et al. 1990) 


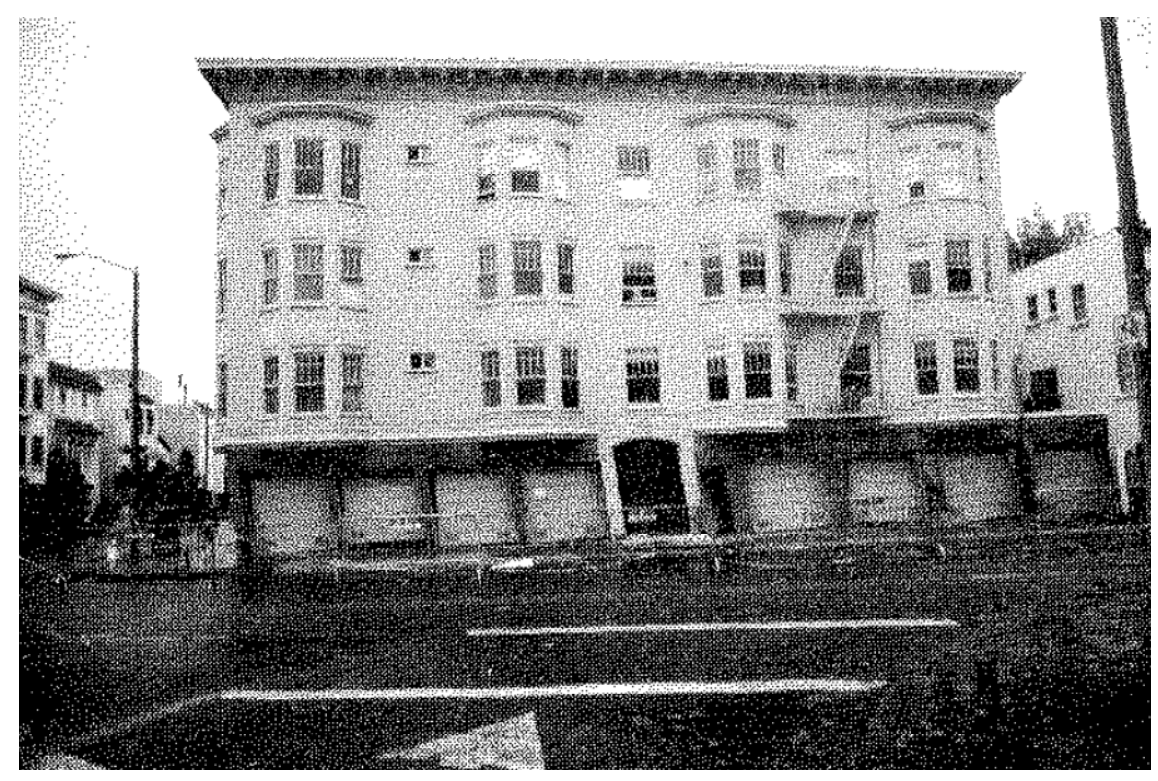

Figure 2-2 More examples of structural damage in the San Francisco's Marina District with "soft" ground floor due to the 1989 Loma Prieta Earthquake (from Seed et al. 1990)

\subsubsection{Components of the Soil-Foundation-Structure Interaction}

The concept of soil-foundation-structure interaction (SFSI) was explained briefly in Chapter 1 . There are three primary components of SFSI effects. These include (1) dissipation of energy from the soil-structure system through radiation damping and hysteretic soil damping (foundation damping effects), (2) variation of the character of the ground shaking motion transmitted to the structure (kinematic effect), and (3) lengthening of the system's fundamental response period with the flexible foundation effects due to the flexibility of the soil-foundation system (inertial effect) (Stewart et al. 1999a). All these effects contribute significantly to the overall design consideration. To fully grasp the causes and effects of SFSI, concepts of kinematic and inertial interactions are presented in the following sections.

\subsubsection{Kinematic Interaction}


Generally, the base-slab motion is considered to match the free-field motion for seismic design. The term "free-field" refers to the motion at the foundation-soil interface in the absence of any structural influence. In reality, the base-slab motion can be different from the free-field motion due to kinematic interaction when a stiff foundation is on or embedded in the soil. The actual motion at the foundation base level is known as the foundation-input-motion (FIM), which is the motion the soil-foundation interface would experience if the foundation was massless; this is more appropriate to use for seismic structural response analysis than to use the free-field motion. The kinematic interaction effects can be quantified by transfer functions, which are the ratios of the FIM to the freefield motion as found in many past SSI analytical studies. The FIM deviates from the free-field motion as a result of kinematic interaction, which is caused by base-slab averaging and embedment effects (Stewart et al. 1998).

Base-slab averaging results from the incoherent or inclined waves from the source across the contact interface of the foundation. The incoherent wave paths are developed as a result of seismic waves traveling laterally through underlying medium and varying propagation paths through different soil materials. The presence of the wave field reduces the translational base-slab motion relative to the free-field motion horizontally and further influences the rotational component of the motion by introducing torsional rotation. Foundation rocking can also occur as a result of inclined wave paths. The reduction of the base-slab horizontal component, along with the additional torsion and rocking, are results of the base-slab averaging effects. The intensity of the base-slab averaging effects tends to become more prominent at lower periods when the ground motion becomes more incoherent. In addition, the increased size of the foundation, 
relative to the seismic wavelength at a lower period, contributes to the base-slab averaging effects (Stewart et al. 1998).

It is not the intent here to present and summarize the past studies of kinematic interaction effects. However, analytical solutions for base-slab averaging effects have been developed by Luco and Mita (1987) and Veletsos and Prasad (1989) for circulation foundations. Furthermore, Luco and Wong (1986) and Veletsos et al. (1997) improved upon the formulation and provided solutions for rectangular foundations. These analytical formulations quantify the transfer functions associated with the ratio of base-slab motion to the free-field motion.

Embedment effects are associated with degree of ground motion propagation relative to the distance away from the earthquake source. Embedded foundations experience reduced base-slab translational motions relative to the free-field motion. The foundation-input- motion can amplify significantly for those foundations located in soft soils as observed in the Mexico and Loma Prieta Earthquakes. This phenomenon has been validated through the studies of Seed and Lysmer (1980) by using SHAKE, a computer program for conducting linear seismic response analyses of horizontally layered soil deposits (Schnabel et al., 1972). In addition to reduction of base-slab translational motion relative to free-field motion, embedded foundations can also experience rocking motions. However, unlike the rocking produced by the structural inertia, the rocking associated with embedment is caused by incompatible shear strains along the sides of the excavation and the free-field (Stewart et al. 1998). Generally, 
embedment effects are only considered for deep foundation systems. For shallowly embedded foundations, embedment effects are not as significant as other SFSI effects.

For embedded structures, foundation translations are reduced relative to the freefield due to the de-amplification of ground motion with depth, along with the wave scattering effects. Elsabee and Morray (1977) utilized a finite soil layer to compare the transfer functions of various foundation embedment ratios (e/r), which is the ratio of embedment to the effective radius of the foundation, while Day (1977) incorporated the elastic half space approach in his finite element analyses. Roesset (1980) concluded that the embedment effects were more considerable for e/r greater than 0.15 . Furthermore, Stewart et al. (1998) suggested that no significant effects were observed for surface and shallowly embedded structures for e $/ \mathrm{r}<0.5$. For this particular study, the kinematic embedment effect is also considered in detail since scale model mat foundation is embedded in the soft model soil.

\subsubsection{Inertial Interaction}

The focus of this thesis is the inertial interaction effect on foundations on soft soils, which can be more significant for foundations with shallow embedment or smaller base-slab (Stewart et al. 1999a). The dynamic responses of structure located on soft clay may be different from the responses of those supported on stiffer soil. According to Veletsos and Meek (1974), the difference is contributed from two main factors:

1) Due to the flexible nature of the supporting medium, the structure supported by soft soil has more degrees of freedom than those on firm ground. 
2) The vibration energy associated with the structure from dynamic loading may be dissipated through radiation of waves through the surrounding soil or by the damping of the foundation element.

Inertial interaction is the principal influence on these factors, which can be quantified by various analytical methods and are presented in the following section.

As described previously, inertia developed in the structure during dynamic excitation from its own vibration creates additional base shear and moment, which result in further displacement and rocking relative to the free-field. The compliance of the soft soil enables the relative displacement and rotation, which alters the overall structural flexibility as oppose to the structures supported by firm ground, or a fixed-base scenario. Furthermore, the inertia in the structure allows for additional system damping by energy dissipation via radiation damping and hysteretic soil damping. Since these effects developed as a result of structural inertia, they are known as the inertial interaction effects. It is well established that the fundamental period of the structure interacting with the soil increases due to inertial interaction (Aviles and Perez-Rocha 1996). In addition to the theoretical FIM from kinematic interaction, the deviation of base-slab motion from free-field motion, caused by inertial interaction, must be considered to fully quantify the SFSI effect. However, the effects of inertial interaction are normally more prominent than the effects of kinematic interaction (Kramer 1996).

In SFSI analyses, the stiffness and damping associated with the foundation-soil interaction are often quantified as the impedance function. Impedance function is a frequency-dependent complex matrix that relates the force, displacement, rotation, and 
damping of the foundation relative to the free-field. Simply put, impedance function can be described as the springs and dashpots at the base of the foundation that quantify transitional and relational deformation relative to free-field. The specific techniques for impedance function calculation are not reviewed herein. However, various methods of SFSI analyses, particularly for inertial interaction, are presented in the following section.

\subsection{Methodologies for Soil-Structure Interaction Analysis}

During a strong earthquake loading, there are two innate nonlinearities associated with SFSI effects. The first is the primary nonlinearity resulting from the deformation of the free-field soil from seismic waves. The nonlinearity of the soil material reduces the shear strain modulus of the deformed soil and increases hysteretic soil damping with additional energy dissipation. The degree of nonlinearity is more significant with greater soil deformation and a larger level of ground shaking. The secondary nonlinearity arises from the stress caused by the vibration and oscillation of the structure on the surrounding soil during shaking. Secondary nonlinearity may be an important factor for heavy or larger buildings, such as life-lines and underground structures (Pitilakis and Clouteau 2009).

Generally, two methods, the direct method and the substructure method, are available for analyzing SFSI. In the direct method, the entire soil, foundation, and structure are considered as one system and analyzed in a single step. The substructure method is the more favorable approach for SFSI analysis because it divides the total soilfoundation-structure domain into several sub-domains and is a fairly effective and straightforward approach to analyze and interpret SFSI. (Kramer 1996). Additional details of each method and its alternatives are presented in the following sections. 


\subsubsection{Direct Analysis Methods}

As stated previously, the direct method considers the entire soil and structure within the same model and analyzes the system in a single step. The finite element method (FEM) and the boundary element method (BEM) are the most popular approaches to provide intensive SFSI analysis. Numerical modeling of the soil and structural elements together takes into account the nonlinear soil behavior, structural and material irregularity, and possible contact interface interaction, such as foundation uplift or sliding (Preisig and Jeremic 2005). The direct method can most accurately capture the behavior of the system under seismic loading, while the substructure method does not yield predictions as precisely as the direct method, due to its simplicity and assumptions.

Finite element numerical models can evaluate the SFSI effects in both time and frequency domains. For time domain, both linear and nonlinear soil behaviors can be captured by incorporating specific soil constitutive properties and laws. However, numerical codes, such as ABAQUS (Pitilankis et al. 2008), which performs both linear and nonlinear analyses, are not specialized for SFSI analysis. The results of the analyses are highly sensitive to soil parameters for the constitutive model (Stewart et al 1998). Moreover, the three-dimensional nonlinear analyses are very complicated, expensive in computational terms, and have issues with adequately simulating the radiation condition of the wave field dissipating away from structure (Pitilakis and Clouteau 2009). Therefore, frequency domain direct finite element codes specifically designed for SSI analyzes, such as FLUSH (Lysmer et al., 1975) and SASSI (Lysmer et al., 1981), are preferred to approximate the linear soil behavior. 
Borja et al. (1999) developed a 3-D finite element model to study the effects of nonlinear soil-structure interaction using time domain analysis techniques. The numerical model was utilized to study the nonlinear seismic response of a large scale seismic test site in Lotung, Taiwan. The finite element model mesh is illustrated in Figure 2-3. A 1/4 scale nuclear plant structure located on the eastern edge of Taiwan was subject to several earthquakes throughout the years, and the data recorded from the site provided a good baseline calibration for the finite element model. The effects of SFSI were determined to reduce the peak ground surface acceleration.

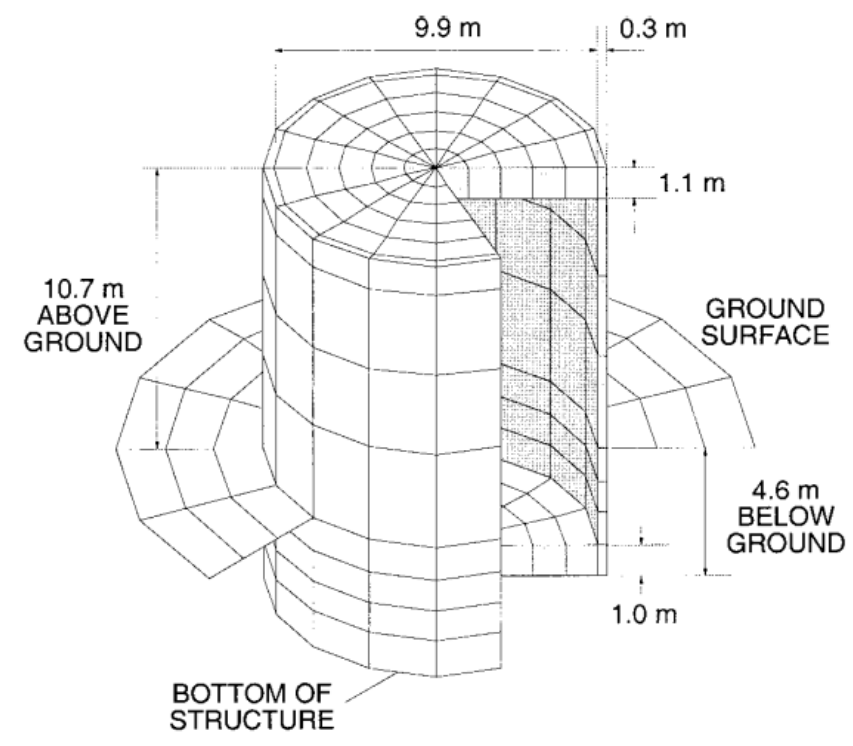

Figure 2-3 Cut out FE mesh of the model in Taiwan (From Borja et al., 1999) Preisig and Jeremic (2005) developed a direct nonlinear SFSI analysis using 3D finite element model. The simulation was created using OpenSees, the open source finite element platform developed by Mazzoni et al. (2000. The nonlinearity of the soil behavior, structure and the interface were considered, along with appropriate radiation conditions for the model. The effects of SFSI were examined by comparing the modeling 
results of both fixed-base and flexible-base scenarios. The analyses were conducted for both 2-D and 3-D models. The 3-D model is illustrated in Figure 2-5 below. The foundation displacement and moment data of for the simulations were compared, and the 2-D modeling was sufficient for evaluating the influence of SFSI.

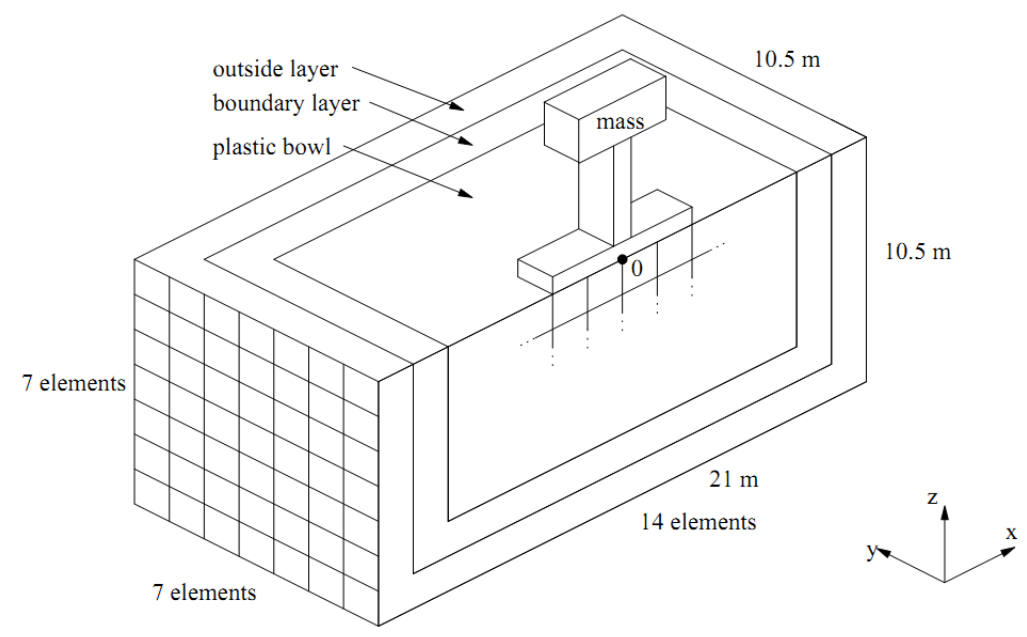

Figure 2-4 The full 3-d model of the OpenSees FEM analysis (From Preisig and Jeremic 2005)

Buehler et al. (2006) evaluated the role of potential liquefiable soil and cyclic mobility for saturated soil on SFSI considerations. The numerical model considered wave propagation and soil liquefaction for free-field condition. Subsequently, SFSI effects during seismic loading were modeled for shallowly embedded and pile foundations for soil prone to liquefaction. The direct approach for SFSI analysis allowed a comprehensive evaluation of the entire building, foundation, and soil system as one. In addition, the displacement, settlement, and rocking of the structure were predicted using the numerical model. This study investigated the nonlinear soil behavior of SFSI by constructing FE mesh using ABACUS as shown in Fig. 2-5. In comparison to shallow embedment, pile foundation buildings experienced less settlement and rocking from the 
modeling results. This proposed FE model accounted for liquefaction potential in the soil, thus allowing a true nonlinear SFSI analysis.
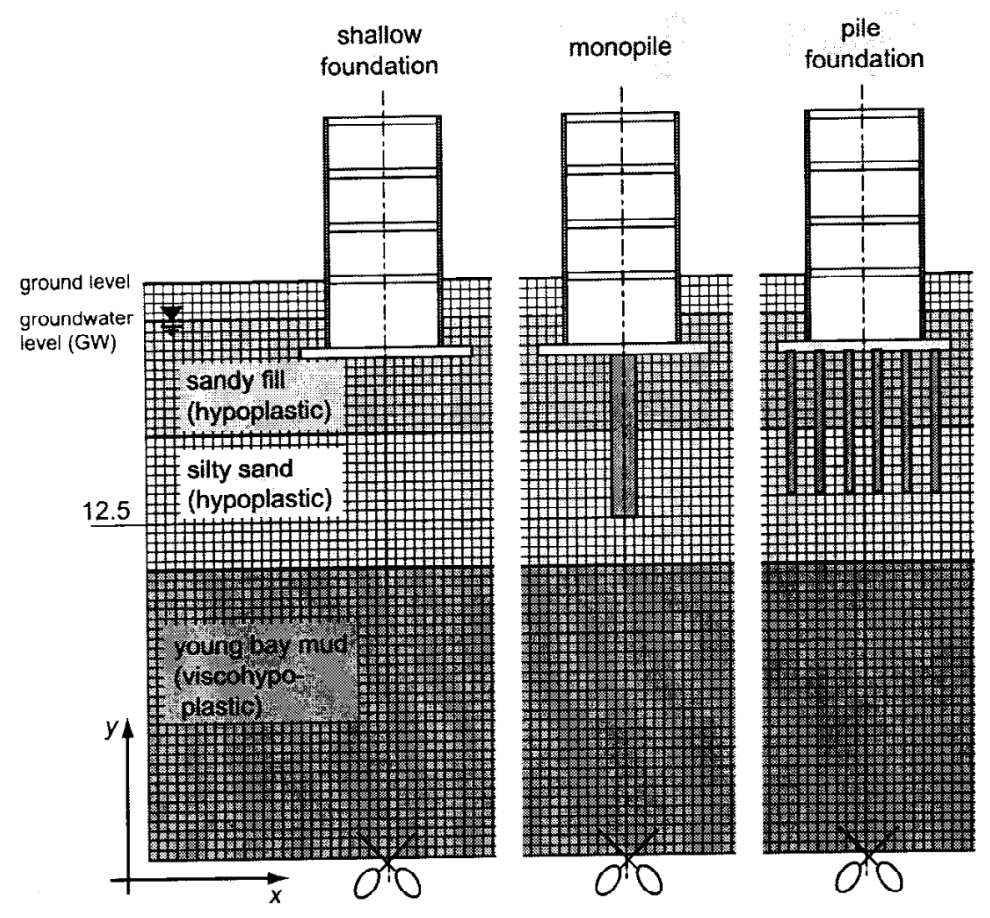

free-
field
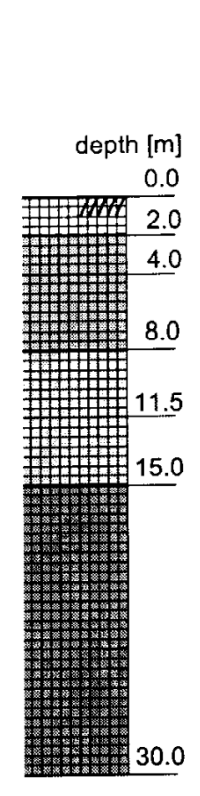

Figure 2-5 The four cases of FE models used for comparative study of SSI effects on liquefiable soils: shallow foundation, monopole foundation, deep pile foundation, and free-field (from Buehler 2006)

Rayhani and Naggar (2008) developed a 3-D finite-difference-based fast Lagrangian analysis numerical model, which was used to validate and examine the effects of SFSI and nonlinear site responses of rigid foundation in soft soil during seismic loading. The program FLAC3D (Itasca 2005) was utilized to develop the numerical model and to simulate the responses during seismic excitation. The nonlinear behavior of the soil was defined by the Mohr-Coulomb criteria, and the model grid is shown in Fig. 2-6. The predicted numerical model results were initially calibrated against the actual data from centrifuge testing. Once verified, further numerical simulations were conducted with different parameters and various intensity shaking motions to determine the effects 
of soil layering, depth, and embedment. The numerical results supported the conclusion that the embedment of structure decreases the amplitude of the response spectra significantly and reduces the structural loads as accompanied by an increase in energy dissipation.

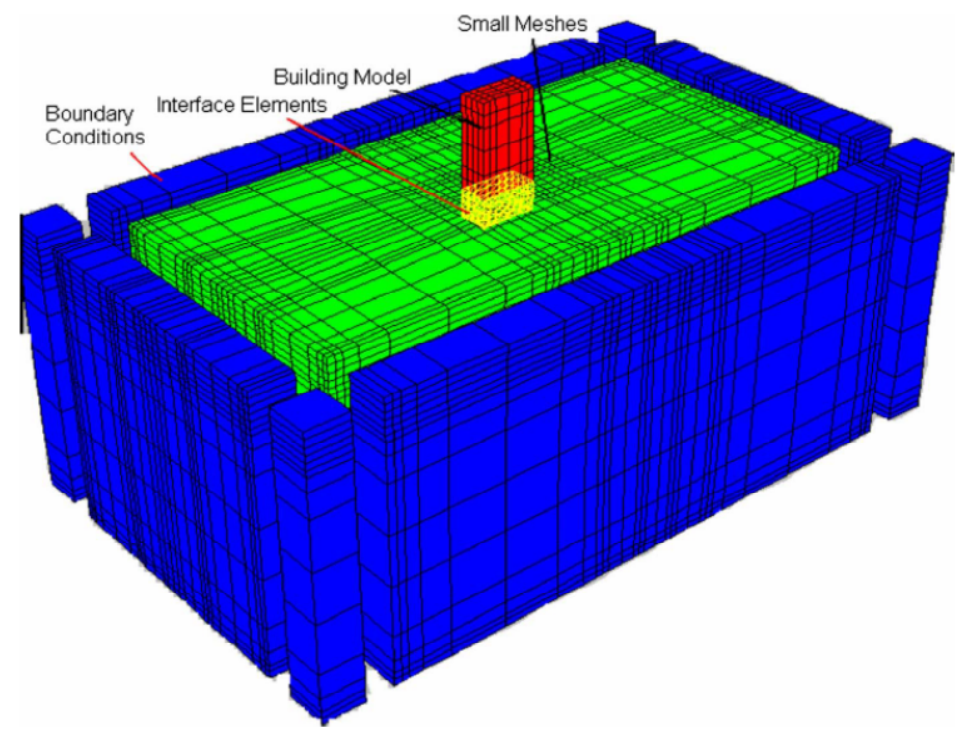

Figure 2-6 Numerical grid and model component in FLAC3D (from Rayhani and Naggar 2008)

\subsubsection{Substructure Analysis}

In the direct method, the number of dynamic degrees of freedom of the soil boundary is significant and results in a complicated finite element model and in considerable computational time. On the contrary, the substructure method, and its associated simple impedance function procedure, can adequately simulate the effects of the nonlinear behavior of the soil in a SFSI analysis. Substructure method has become widely used in practice for its simplicity and effectiveness (Wolf 1985). Originally developed by Veletsos and Meek (1974) and Bielak (1975), several analytical approaches 
exist for the analysis for each subdomain. The complexity of the models ranges from the simple equivalent mass-spring-dashpot system to complicated finite element models.

In the substructure method, the SFSI solution is divided into three separate issues to expedite the computation process. The results of the three parts are then combined to formulate the complete analysis as summarized by Stewart et al. (1998). Assumptions regarding the soil and structural linear behaviors are necessary to adhere to the law of superposition. The three steps in the substructure method are illustrated in Fig. 2-7 and explained as follows:

1. Evaluation of the Foundation Input Motion (FIM), which was previously described as the potential motion on the base-slab if the structure and the foundation were massless. The theoretical FIM is analyzed based on the stiffness and the geometry of the foundation and the soil. Inertial effect is neglected since FIM represents the effects of kinematic interaction only.

2. Determination of the impedance function. As stated earlier, impedance function represents the stiffness and damping characteristics of SFSI. The function accounts for the stiffness and geometry for both the soil stratigraphy and the structure. The analytical method of computing the impedance function is based upon equivalent-liner soil properties.

3. Full dynamic system analysis of the structure supported on a base represented by the impedance function and subjected to base loading from the FIM.

The substructure method provides the best flexibility in the overall analysis procedure. By breaking down the substructure method into three steps, attention can be focused on the most significant aspect of the analysis. 


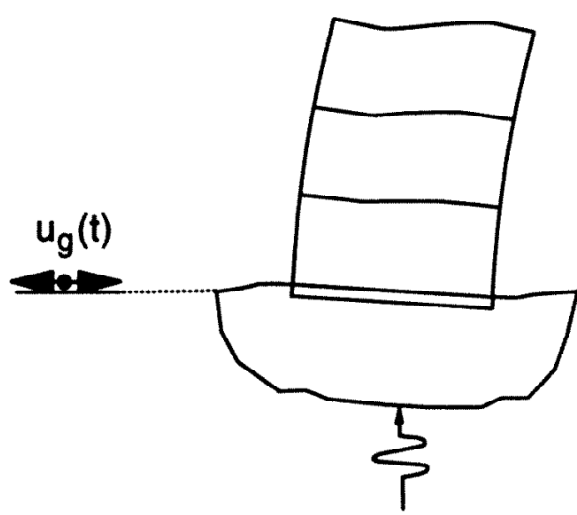

Interaction Problem

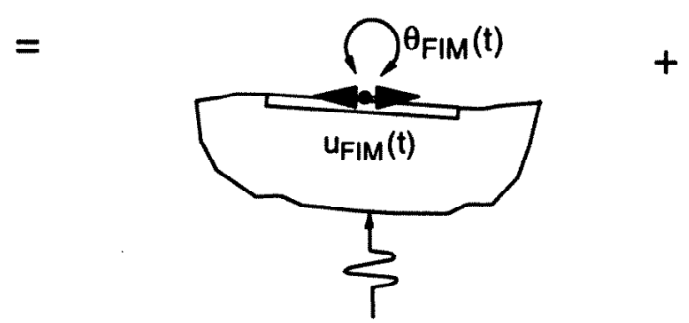

(1) Kinematic Interaction, Evaluation of Foundation Input Motions

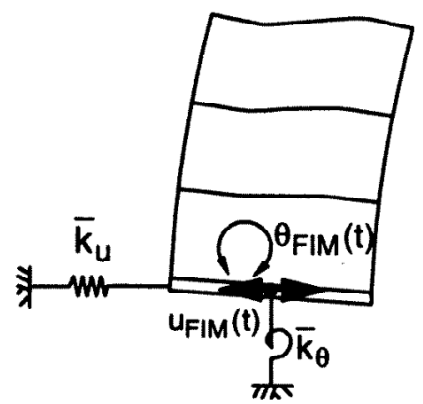

(3) Analysis of structure on compliant base subjected to FIM

Figure 2-7 Substructure method to analysis of the SFSI by breaking down into three steps (from Stewart et al. 1998)

In Step 1, the kinematic interaction is quantified as transfer function amplitudes that relate foundation and free-field motion. The methods to analyze the transfer functions are mentioned in the previous section. However, the kinematic interaction aspect of the SFSI system has been traditionally ignored. According to SFSI provisions in the Applied Technology Council (ATC 1978) and the National Earthquake Reduction Program (NEHRP) (BSSC 1997) seismic codes, kinematic interaction effects are generally ignored, and the free-field motion and FIM are assumed to be the same in the 
code provisions. The analytical methods for inertial interaction effects are the primary focus of this section.

Simplified analytical formation has been developed for the inertial interaction effects for the substructure method. In general, the inertial interact effect analyses calculate the variation of the first-mode period and the damping ratio between the fixedbase and flexible-base cases. Steps 2 and 3 of the substructure method consider the flexible-base system as a whole and analyze the modal parameters with the free-field response spectrum to evaluate the necessary design base shear forces for the structure (Stewart et al. 1998). A simplified system typically used in analyses for inertial interaction is shown in Fig. 2-8.

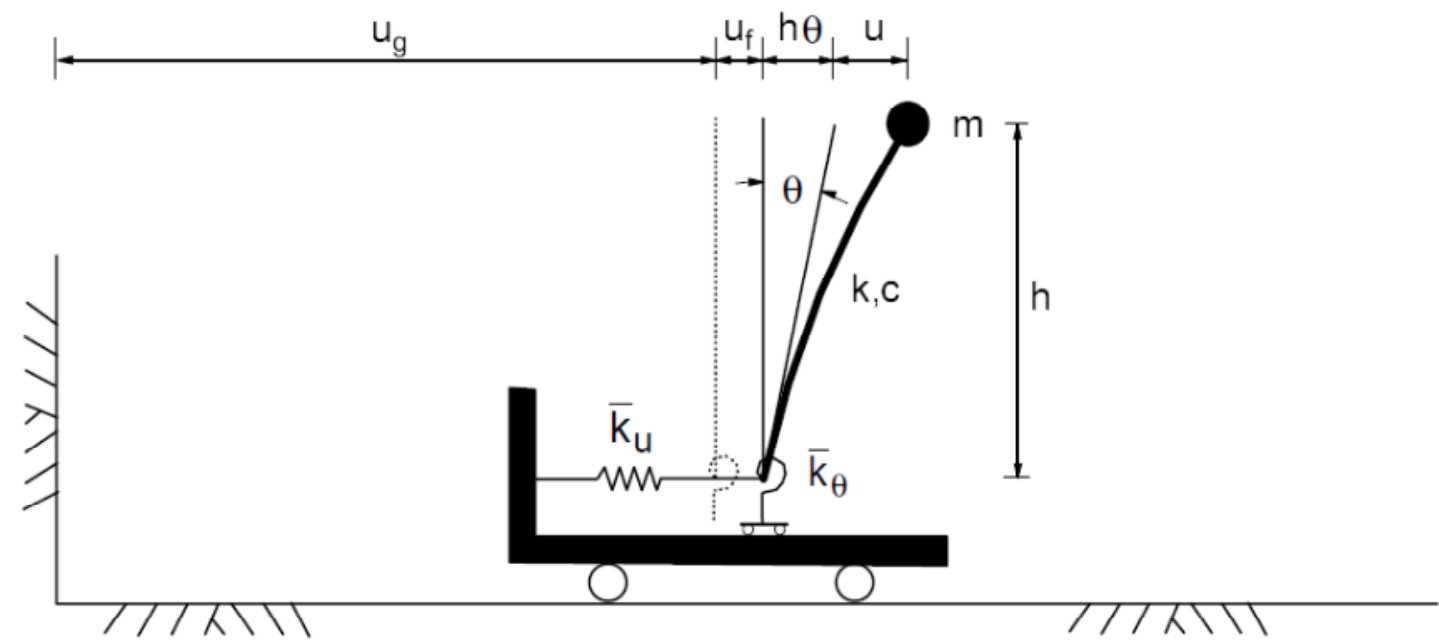

Figure 2-8 Simplified model for inertial interaction analysis (from Stewart et al. 1999a) The simple equivalent mass-spring-dashpot system originated from the works of Veletsos and Meek (1974) and Bielak (1975). The concept is later employed by Gazetas (1986) and Stewart et al. (1999a). The system is considered as a single degree-of-freedom structure with height $h$, mass $\mathrm{m}$, stiffness $\mathrm{k}$, and viscous damping coefficient $\mathrm{c}$. Translational and rotational displacement relative to the free-field behavior at the base of the structure are represented by $\mathrm{u}_{\mathrm{f}}$ and $\theta$, respectively. The impedance function is 
modeled by a flexible foundation medium, represented by the frequency dependent and complex value translational and rotational springs $\overline{\mathrm{k}_{\mathrm{u}}}$ and $\overline{\mathrm{k}_{\theta}}$. As presented previously, the impedance function relates the force and moment applied at the base of the structure to the displacements and rotations of the foundation relative to the free-field. In this model, the building is often viewed as a single-story building and can also be approximated as the model of a multistory building that is dominated by the fundamental mode response. In the case of a multistory building, $\mathrm{h}$ is identified as the distance from the base to the centroid of the inertial forces from the first mode vibration (Stewart et al. 1998).

A few analytical procedures for impedance function estimates are outlined and reviewed in Luco (1980) and Roesset (1980). The more commonly used solution was developed by Veletsos and Wei (1971) and Veletsos and Verbic (1973) for rigid circular foundation on the surface of a visco-elastic halfspace. As shown in Fig. 2-7, the lateral response of a structure on a rigid foundation becomes a six degree-of-freedom system that can be described in two impedance functions. According to Stewart et al. (1998), the simple impedance function is expressed in Eq. 2.1.

$$
\left[\begin{array}{l}
V \\
M
\end{array}\right]=\left[\begin{array}{cc}
\overline{k_{u}} & 0 \\
0 & \overline{k_{\theta}}
\end{array}\right]\left[\begin{array}{c}
U_{f} \\
\theta
\end{array}\right]
$$

Where $\mathrm{V}$ is the base shear, $\mathrm{M}$ is the base moment, $\overline{\mathrm{K}_{\mathrm{u}}}$ is the impedance function of the translational spring, $\overline{\mathrm{K}_{\ominus}}$ is the impedance function for the rotational spring, $\mathrm{U}_{\mathrm{f}}$ is the lateral displacement, and $\theta$ is the rotation of the structure. For the solution of a rigid disk on a halfspace, impedance functions are expressed in Eq. 2.2.

$$
\bar{k}_{J}=\bar{k}_{J}\left(a_{0}, v\right)+i w c_{j}\left(a_{0}, v\right)
$$


Where $\mathrm{j}$ denotes either deformation mode $\mathrm{u}$ or $\theta, \omega$ is angular frequency (radians/sec), $v$ is the soil Poisson ratio, $a_{0}$ is a dimensionless frequency defined by Eq. 2.3.

$$
a_{0}=\omega r / V_{s}
$$

The foundation radius is expressed as $r$, and Vs is the soil shear wave velocity.

Foundation radii are computed separately for translational and rotational deformation modes to match the area $\left(\mathrm{A}_{\mathrm{f}}\right)$ and the moment of inertia $\left(\mathrm{I}_{\mathrm{f}}\right)$ of the foundation in the following equations.

$$
\begin{gathered}
r_{1}=\sqrt{\frac{A_{f}}{\pi}} \\
r_{2}=\sqrt[4]{\frac{4 * I_{f}}{\pi}}
\end{gathered}
$$

In addition to the separate foundation radii for the different modes, there are different corresponding values for $\left(\mathrm{a}_{0}\right)_{1}$ and $\left(\mathrm{a}_{0}\right)_{2}$ as well.

The actual stiffness and damping of the system are expressed in terms of translational and rotational springs and dashpots, respectively as follows,

$$
\begin{gathered}
k_{u}=a_{u} k_{u} \\
C_{u}=\beta_{u} \frac{k_{u} r_{1}}{V_{s}} \\
k_{\theta}=a_{\theta} k_{\theta} \\
C_{\theta}=\beta_{\theta} \frac{k_{\theta} r_{2}}{V_{s}}
\end{gathered}
$$

Dimensionless parameters $a_{u}, \beta_{u}, a_{\theta}$, and $\beta_{\theta}$ are frequency-dependent values that express foundation stiffness and damping factors for elastic and visco-elastic half-spaces based on closed form expressions in Veletsos and Verbic (1973). The corresponding frequency 
dependent values for $v=0.4$ is shown in Fig. 2-9. The static stiffness of a disk on a halfspace can be defined by $\mathrm{K}_{\mathrm{u}}$ and $\mathrm{K}_{\theta}$, which are expressed as

$$
\begin{gathered}
K_{u}=\frac{8}{2-v} G r_{1} \\
K_{\theta}=\frac{8}{3(1-v)} G r_{2}^{3}
\end{gathered}
$$

Where $\mathrm{G}$ is the soil dynamic shear modulus.
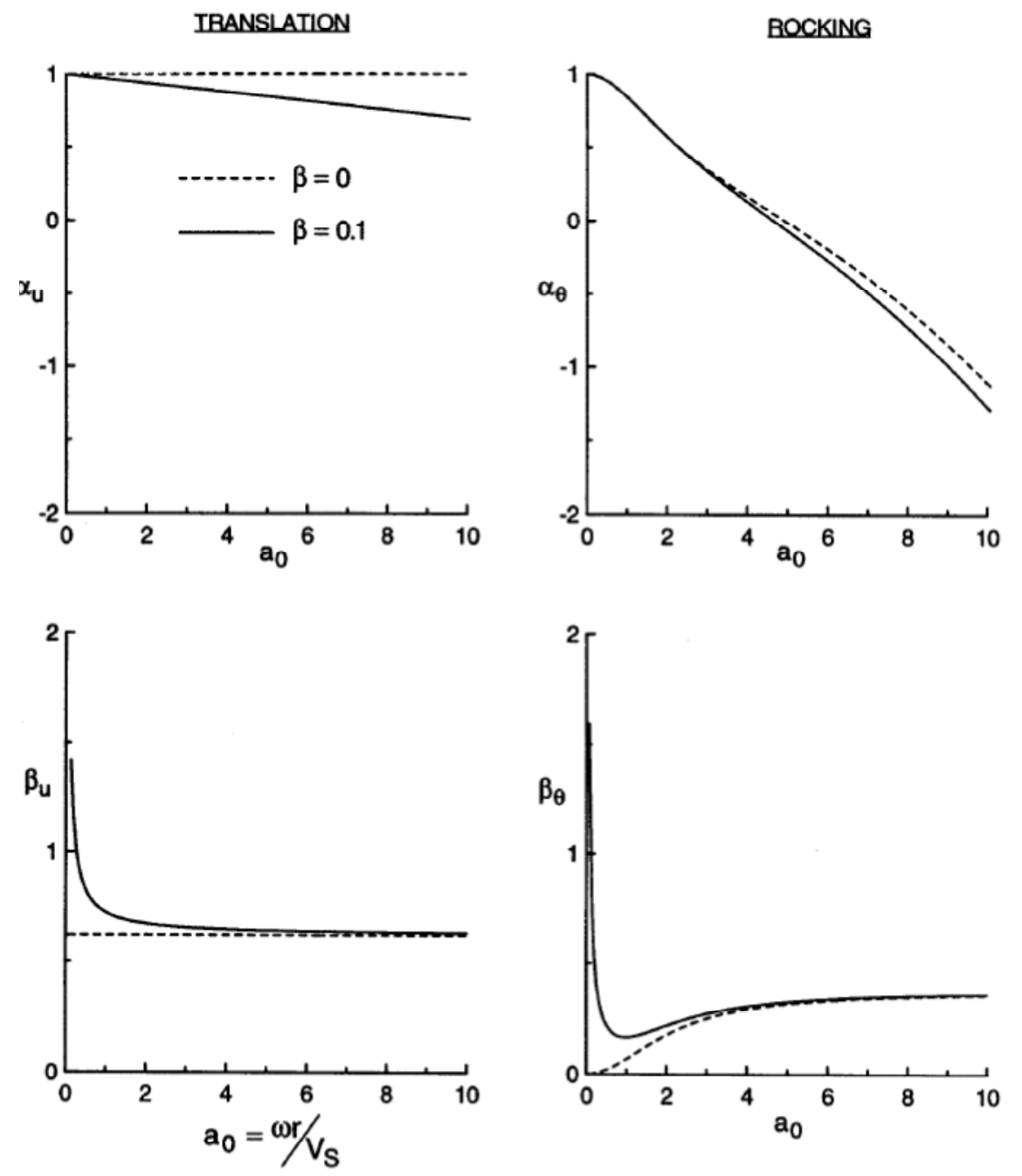

Figure 2-9 Foundation stiffness and damping factors for elastic and viscoelastic halfspaces, $v=0.6$ (after Veletsos and Verbic (1973) and Stewart et al. (1998)) 
Lin and Jennings (1984) have conducted validation studies by imposing sinusoidal ground vibrations to a $10 \times 10$ foot model structure located 50 feet from the point of excitation. A layout of the experiment is shown in Fig. 2-10. The model structure was embedded to determine the effect of foundation embedment on the impedance functions, which were then calculated from the fundamental resonant frequency. The results were compared to theoretical values, and it was determined that the unembedded case yielded agreeable results to the analytical formulations.

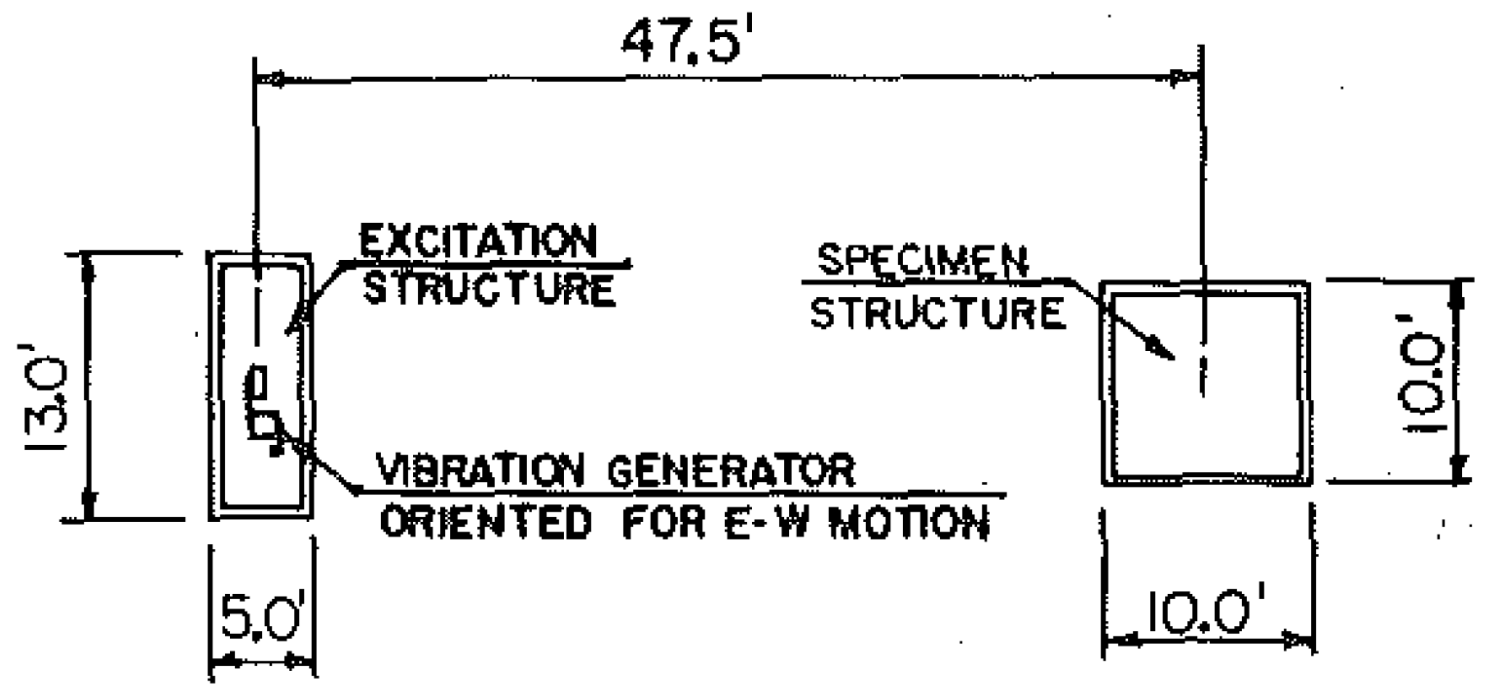

Figure 2-10 Schematic plan of experimental site (from Lee and Jennings 1984)

To analyze the transfer function of embedded foundations, Elsabee and Morray (1977) and Day (1977) studied the relation of base-slab transitional for a circular foundation embedded at certain depth e into a homogeneous soil layer of depth $d_{s}$. The system is shown in Fig. 2-11. The increase of static stiffness from the embedment 
resulted in changes in the frequency-dependent variations of stiffness and damping. The increase of stiffness can be expressed as follows for $\mathrm{r} / \mathrm{d}_{\mathrm{s}}<0.5$ and $\mathrm{e} / \mathrm{r}<1$ :

$$
\begin{aligned}
& \left(K_{u}\right)_{F L / E}=K_{u}\left(1+\frac{2 e}{3 r}\right)\left(1+\frac{5 e}{4 d_{s}}\right)\left(1+\frac{e}{2 d_{s}}\right) \\
& \left(K_{\theta}\right)_{F L / E}=K_{\theta}\left(1+\frac{2 e}{r}\right)\left(1+\frac{0.7 e}{d_{s}}\right)\left(1+\frac{e}{6 d_{s}}\right)
\end{aligned}
$$

Where $\left(K_{u}\right)_{F L / E}$ and $\left(K_{\theta}\right)_{F L / E}$ are the static horizontal and rocking stiffnesses of the embedded foundation on finite soil layer. For small embedment ratios $(\mathrm{e} / \mathrm{r}<0.5)$, coupling impedance terms were small, relative to $\left(K_{u}\right)_{F L / E}$ and $\left(K_{\theta}\right)_{F L / E}$. Elsabee and Morray (1977) suggested that foundation stiffness and damping were frequency dependent and thus may be approximated from Eq. 2.5 to 2.8. This approach was also adopted in the NEHRP (BSSC 1997) code provisions for soil-foundation-structure analysis.

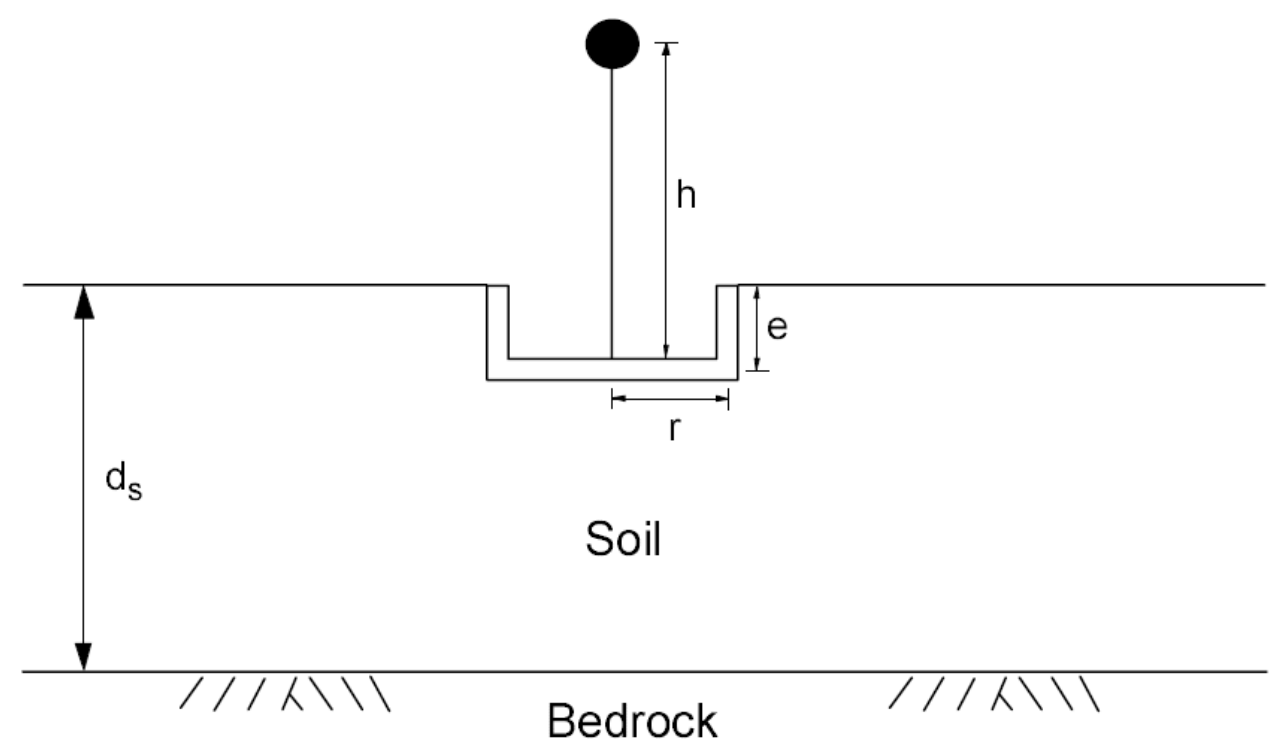

Figure 2-11 Embedded soil-foundation-structure system on finite soil layer for Elsabee and Morray's analysis (after Elsabee and Morray (1977) and Stewart et al. (1998)) 
For practical application and analysis, numerous studies have developed tabular or graphical solutions for impedance function specific for various conditions. For surface foundations, Veletsos and Wei (1971) and Luco and Mita (1987) have developed tabular solution for circular foundations, and Luco and Wong (1986) incorporate a uniform layer of half-space for the rectangular and square foundations. Moreover, for embedded foundations, Apsel and Luco (1987) have developed solutions for circular foundations, as Mita and Luco (1989) have done the square foundations.

Conventionally, embedment effects on foundation impedance are evaluated with the equivalent fixed-base single degree-of-freedom oscillator. Veletsos and Meek (1974) evaluated the effects by comparing the fundamental periods and damping ratios of the fixed-based scenario to the flexible base. The flexible base period can be approximated with Eq. 2.11.

$$
\frac{\tilde{T}}{T}=\sqrt{1+\frac{k}{k_{u}}+\frac{k h^{2}}{k_{\theta}}}
$$

Where $T$ is the fixed-base period of the oscillator, which is the period that would occur in the absence of base translation or rocking as shown in Fig. 2-7. $\tilde{T}$ is the flexible-base oscillator period that incorporates the total behavior the system. Regarding damping ratios, Jennings and Bielack (1973) and Veletsos and Nair (1975) approximated the relationship between fixed-based and flexible-base damping ratios as

$$
\tilde{\zeta}=\widetilde{\zeta_{o}}+\frac{\zeta}{(\widetilde{T} / T)^{3}}
$$

Where $\zeta$ is the fixed-base foundation damping ratio, $\tilde{\zeta}$ is the flexible-base foundation damping ratio, and $\widetilde{\zeta_{o}}$ is the foundation damping factor characterized by the hysteretic and radiation damping of foundation-soil interaction. Furthermore, the relationship between 
fixed- and flexible- base single degree-of-freedom oscillator properties can be influenced by the aspect ratio $\left(\mathrm{h} / \mathrm{r}_{2}\right)$, soil Poisson Ratio $v$, soil hysteretic damping ratio $\beta$, and the ratio of soil-to-structure stiffness $\sigma$, and structure-to-soil mass $\gamma$. The latter two dimensionless factors can be expressed as

$$
\begin{aligned}
& \sigma=V_{s} T / h \\
& \gamma=\frac{m}{\rho \pi r_{1}^{2} h}
\end{aligned}
$$

The typical values for conventional buildings are $\sigma>2$ and $\gamma \approx 0.1$ to 0.2 (Stewart et al.

1998). Values of $\frac{\tilde{T}}{T}$ and $\widetilde{\zeta_{o}}$ are sensitive to $\sigma$, but not as significant for $\gamma$ (Aviles and Perez-Rocha 1996).

The relation of these parameters are compared to analytical results for $\frac{\tilde{T}}{T}$ and $\widetilde{\zeta_{o}}$ vs. $1 / \sigma$ for rigid circular foundation embedded into a visco-elastic soil in Fig. 2-11. The analytical formations used in the comparison plots are presented Veletsos and Nair (1975), Bielak (1975), and Aviles and Perez-Rocha (1996). The Veletsos and Nair formulation and Bielak solution are applicable to foundation embedded in a half-space, while the Aviles and Perez-Rocha solution considers the embedment in a thick finite layer $\left(d_{s} / r=10\right)$. As seen in the plots in Fig. 2-12, the three solutions have similar period lengthening results for the zero embedment; however, the damping from the Aviles and Perez-Rocha model is slightly higher than the others. For the case of embedment ratio of $\mathrm{e} / \mathrm{r}=1$, the three models yield agreeable trends with a reduction of period lengthening and an increase of damping all across the board. Note that, in comparison, the Bielak model yields the highest damping because the Veletsos and Nair model does not account for the dynamic basement wall-soil interaction, and the Aviles and Perez-Rocha model considers the finite soil layer. 

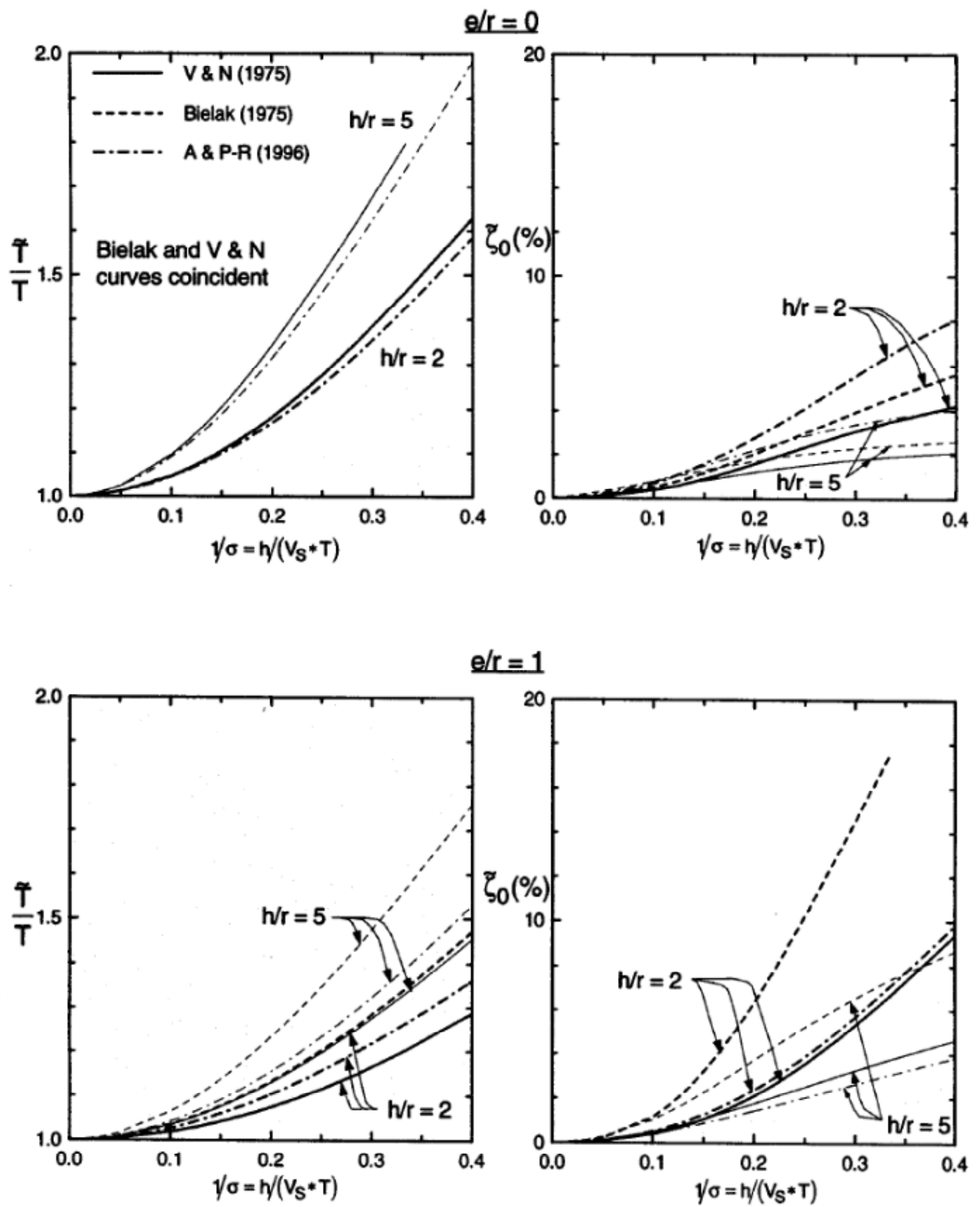

Figure 2-12 Comparison plots of period lengthening ratios and foundation damping factors for single degree-of-freedom structure with surface and embedded foundations $(v$ $=0.45 ; \beta=5 \% ; \gamma=0.15 ; \zeta=5 \%$ ) [after Veletsos and Nair (1975); Bielak (1975); Aviles and Perez-Rocha (1996); Stewart et al. (1998)]

The analytical procedures and equations analyzing the inertial effect of SFSI by predicting the period lengthening ratios and foundation damping factors are presented. In 
the study of Stewart et al. (1998), the Bielak model and the Veletsos and Nair model are utilized with appropriate modifications for effectiveness and accuracy. The empirical SFSI results from the study are compared to the analytical solutions outcome, as presented herein, and the results are presented in a later section.

As mentioned previously, numerical model analyses are appropriate to evaluate the effects of SFSI. The aforementioned numerical modeling solutions are for the direct method, which analyze the entire system and in a single step. In addition, several numerical analytical models incorporate the substructure approach. A brief overview of substructure numerical approach is presented below, although it is not the purpose of this research to construct numerical models using the following methods.

Gajan et al. (2008) addressed the lack of conventional linear-equivalent analyses unable to fully capture the true nonlinear behavior at the foundation level for SFSI phenomenon. These nonlinear behaviors include sliding, energy dissipation from hysteretic effects, foundation settlement, and formation of temporary gap between the foundation wall and the soil. To account for these structural system responses from a performance-based SFSI design approach, two modeling frameworks were developed.

The first tool is the beam-on-nonlinear-Winkler-foundation (BNWF) model that consists of a mesh of closely spaced independent nonlinear inelastic springs, which are able to model gapping and radiation damping. Associated behaviors such as rocking, uplift, and settlement are captured by the vertical springs along the base of the foundation, and the resistance to sliding is captured by the horizontal springs distributed to the side of the foundation. A schematic of the BNWF model is shown in Fig. 2-13. The BNWF model can predict the behavior of structure footing elements with the user- 
specified material stiffness and strength; however, it does not mimic the coupling foundation response in the vertical direction with horizontal response. Ultimately, the BNWF model is the preferred model when designing foundation elements for varying stiffness elements (Gajan et al. 2008).

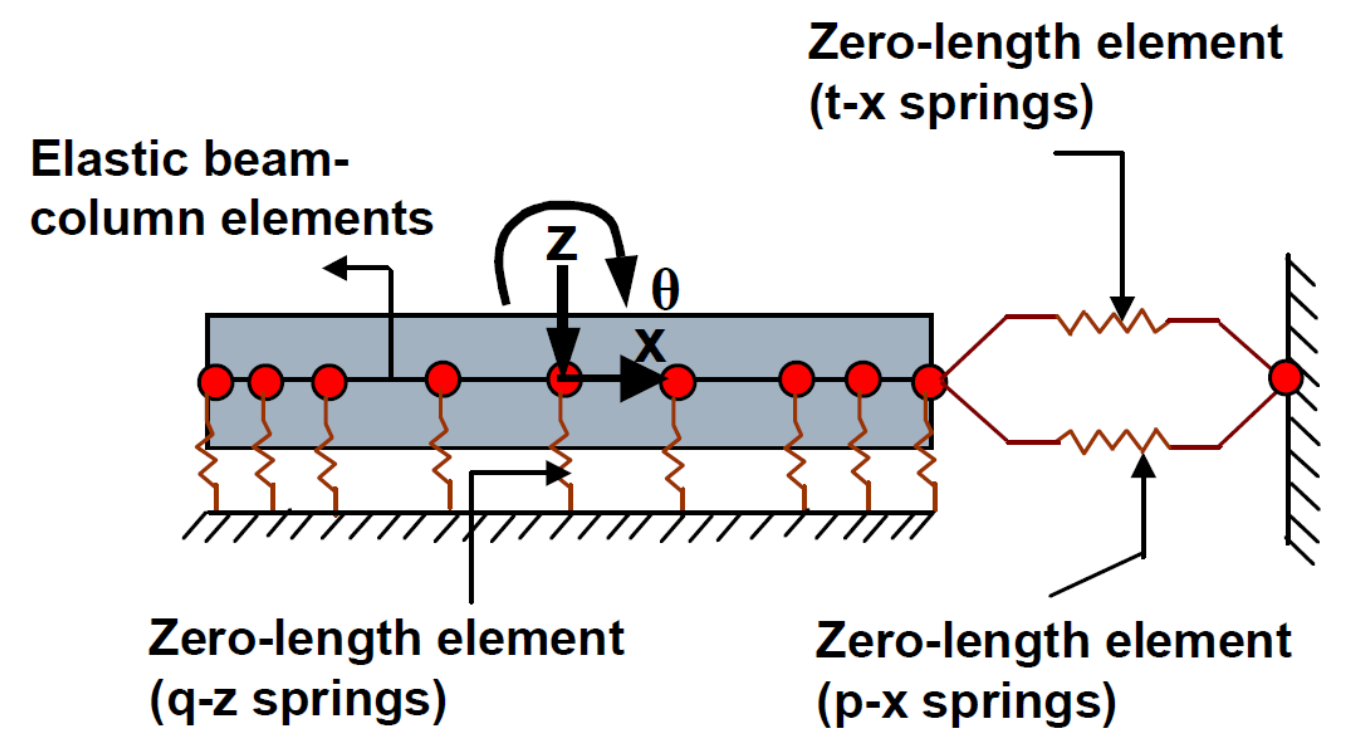

Figure 2-13 BNWF model schematic (from Gajan et al. 2008)

The second tool is the contact interface model (CIM), which accounts for the nonlinear constitutive relations between cyclic loads (axial, shear, and moment) and displacements at the foundation-soil interface for shallow rigid. Unlike the BNWF model, the CIM model couples the foundation behavior in the vertical direction in response to loads with horizontal response. The assumed rigid foundation and the soil below the foundation in the zone of influence are considered to be macro-elements, which are modeled according to the geometry of the soil surface, as well as the kinematic of the foundation-soil system. Consequently, the CIM is the preferred method when considering highly coupled shear and moment responses (Gajan and Kutter 2009). A concept and schematic of CIM is shown in Fig. 2-14. 


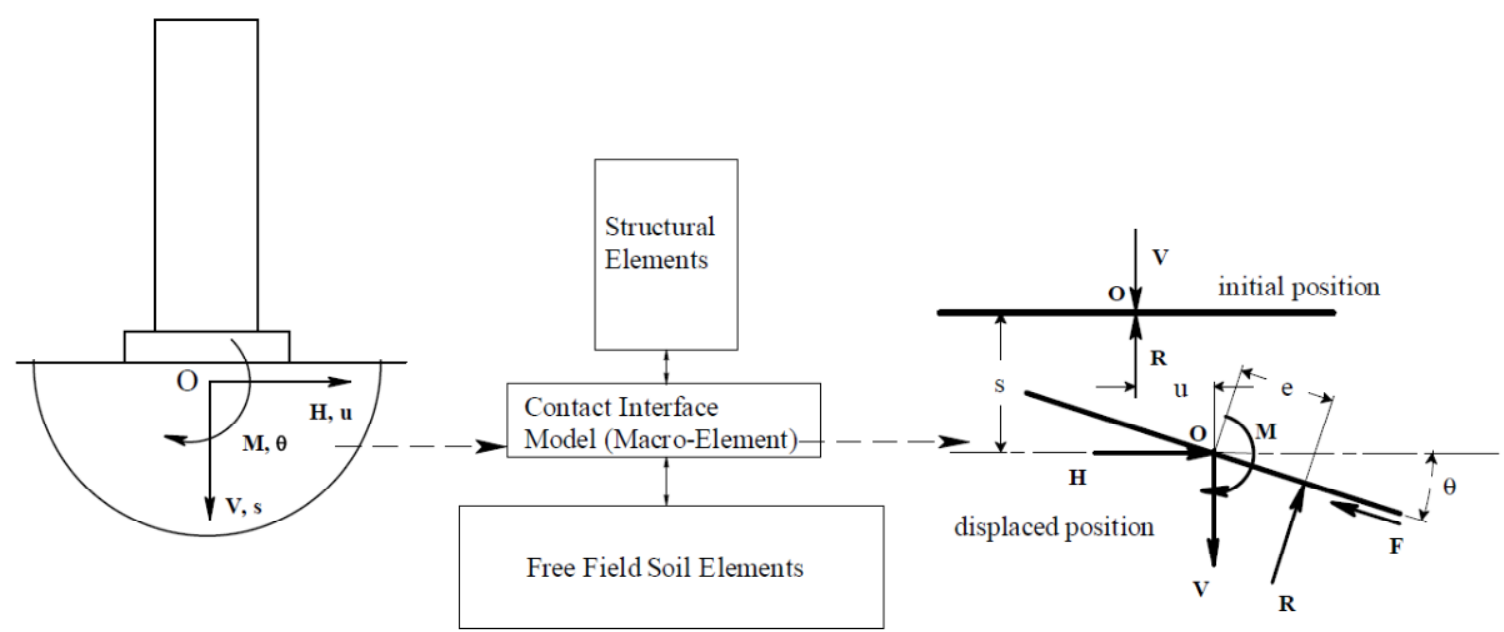

Figure 2-14 Concept of macro-element contact interface model, and the associated forces and displacements at foundation-soil interface during combined loading (from Gajan and Kutter 2009)

Both models allow for user-defined parameters, such as foundation geometry, material shear strength, and soil stiffness. The material properties, as well as the model elements, must be defined and coded using. Recently, many Ph.D. students wrote codes for OpenSees models with well-defined parameters; however, further validation of the BNWF and CIM models results against large scale empirical data are needed for better calibration.

Pitilakis and Clouteau (2009) presented an equivalent linear substructure numerical approximation method for SFSI. Conventionally, the direct numerical finite element approach is the only feasible way of completely accounting for the true nonlinear behavior of the soil in SFSI analysis, but the computational cost for such 3-D full nonlinear SFSI analysis is exponential. However, the simplified yet efficient substructure method produces reliable and realistic analytical results that adequately simulate the nonlinear behavior of the soil in SFSI analysis. Aubry and Clouteau (1992) and Clouteau and Aubry (2003) developed the analytical equivalent linear formulation by incorporating 
the substructure method. Furthermore, this technique was used to create the numerical code MISS3D (Clouteau 2005) in order to conduct SFSI analyses in the linear elastic or viscoelastic domain. The results of the numerical analyses were validated against empirical centrifuge testing of a SDOF situated on coarse grained soil. The newly developed equivalent linear numerical procedure, based on substructure method for SFSI analyses, was validated to simulate adequately the effects of both the primary and secondary nonlinear soil behavior. Therefore, it can potentially substitute the traditional expensive and time consuming FEM numerical analysis at a lower cost and faster execution.

\subsection{Summary}

Two basic methods and associated alternative SFSI analyses can be categorized into direct and substructure methods. In the direct method, the entire soil-foundationstructure system is analyzed in a single step, and often requires complicated numerical finite element models. The computation cost and time can be excessive for the direct method. One the contrary, the substructure method divides the SFSI system into subdomains and then executes the analysis by focusing on the more influential targets. The simplified substructure method yields adequate results in a shorter time; therefore, it has been adopted by the NEHRP code provisions and has become the more commonly used method in practice. In addition, recent studies have incorporated the substructure method into numerical analysis, further validating it as the more efficient SFSI analysis alternative to the full-on direct finite element analysis.

\section{CHAPTER 3 - PREVIOUS EMPRICAL FINDINGS}


With the SFSI analysis theory and concept already well-established, researchers wanted to further understand the influences of various parameters on the overall system performance. However, few empirical studies have been performed, due to the lack of availability of strong motion data from sites with calibrated and properly installed instrumentations in the structure, as well as the free-field. In order to obtain actual empirical data towards understanding the complex problem, studies on SFSI have been conducted both on the field with actual buildings and in the lab with scale models. A few of the notable empirical studies relevant to this thesis are presented below to provide a better overview of the past empirical researches on this subject.

\subsection{Empirical Studies}

Stewart et al. (1999b) collected strong motion data at 57 sites (including building and free-field recordings) in California and Taiwan to evaluate the effects of SSI on seismic structural response. The kinematic interaction effects, or the foundation input motion, at these sites were determined to be relatively insignificant. Rather, the inertial interaction effects, which were quantified by comparing the fixed- and flexible-base modal response parameters, can be significant for certain conditions. The simplified procedures for inertial interaction analysis mentioned previously, along with the system identification procedures for evaluation modal vibration parameters for different cases of base flexibility as outlined in Stewart et al. (1999a), are applied to evaluate the first-mode period lengthening ratios $\frac{\tilde{T}}{T}$ and foundation damping factors $\widetilde{\zeta_{o}}$.

The comparison of the free-field and foundation- level structure motions for the peak acceleration data and corresponding 5\% spectral acceleration at flexible-base 
fundamental period was shown previously in Fig. 1-2. Based on a more detailed comprehensive examination of the empirical and analytical results, the structure-to-soil stiffness has the highest influence on the period lengthening ratio and the foundation damping ratio. While additional factors such as structure's aspect ratio, size, shape, foundation embedment, and flexibility can also affect the inertial interaction at various levels (Stewart et al. 1999b). This study works to provide additional empirical data to support the trend of peak foundation acceleration and spectral acceleration reduction at building fundamental period at deeper embedment depths.

\subsubsection{Shake Table Testing of Shallow Foundation}

Shake table testing has been widely used to examine the influence of dynamic loading on full-scale structural systems; however, for soil-structure interaction study, similitude scaling has often been applied to enable the simulation of a larger soil prototype. Several experimental shake table tests were performed in past studies to simulate the dynamic behavior of shallow foundations during earthquakes.

Shirato et al. (2008) conducted large scale 1-D shake testing on nonlinear behavior of shallow foundation on cohesionless soil. The experimental parameters varied in loading methods, input seismic motions, sandy soil densities, and the ratio of horizontal and overturning moment loads. Acceleration and displacement data of the soil and foundation, as well as the shear force at the base of the foundation, were captured during testing. The experimental data were used to calibrate the numerical model that could simulate coupling effects of the dynamic behavior of the shallow foundation in the study of Paolucci et al. (2008). A picture of the shake table setup is shown in Fig. 3-1. A laminar shear box with dimension of $4 \mathrm{~m}$ x $4 \mathrm{~m}$ x $2.1 \mathrm{~m}$ was filled with compacted Toyoura 
sand deposit. A model shallow pier footing, located at the center of the sand soil surface, was subject to loading. The results from shake table experiments of the model foundation on sand concluded that permanent residual foundation sliding displacement is dependent upon the base excitation intensity.

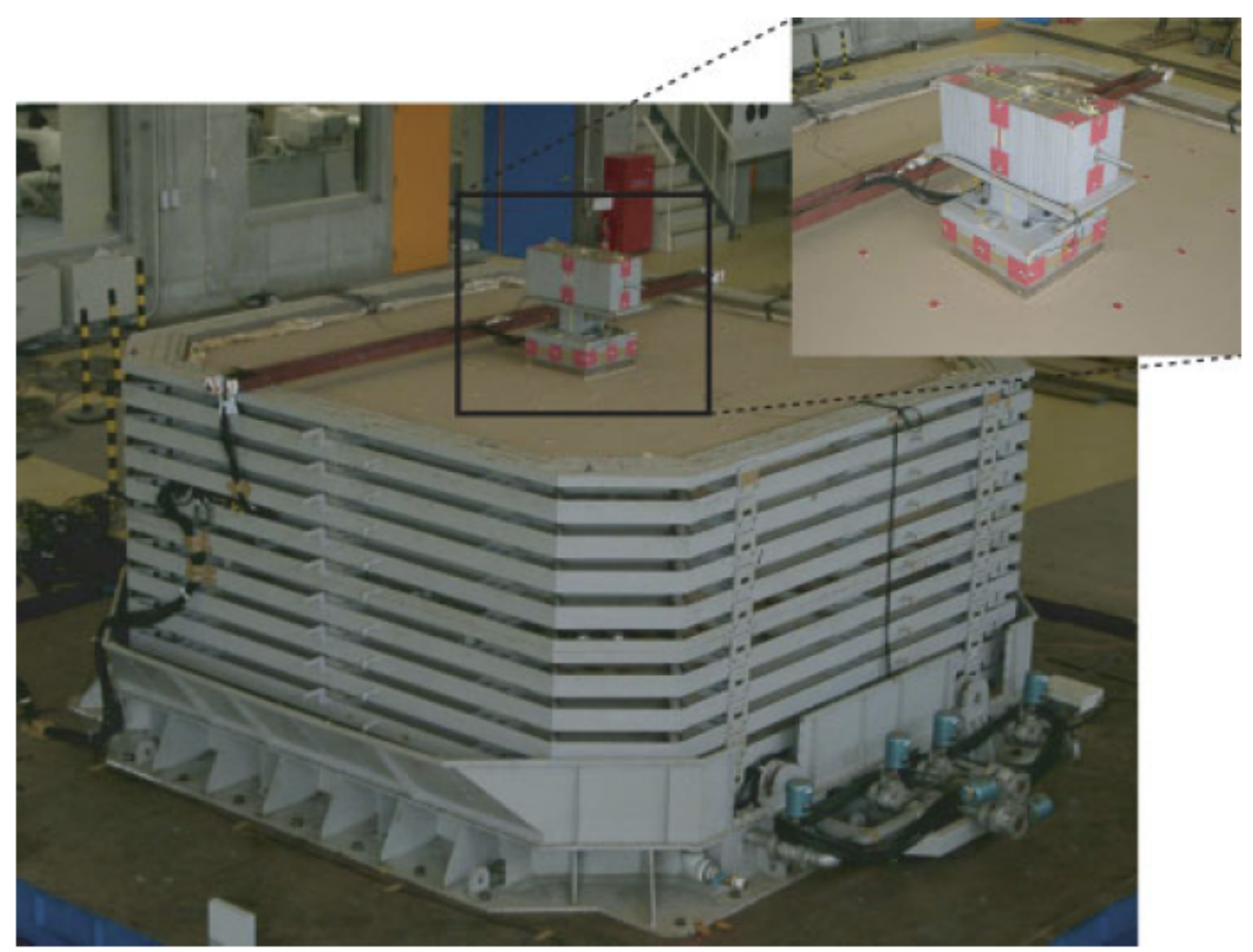

Figure 3-1 Picture of the shake table experiment set-up with a detailed picture of the model foundation located on sand deposit surface (from Paolucci et al, 2008)

In addition, the data served as a bench mark for the numerical modeling developed by Paolucci et al. (2007) for seismic behavior for shallow foundations. Similar to previous SFSI shake table testing, this experiment was conducted using cohesionless soil. Relative displacement and rocking under seismic excitation for the foundations are the main concerns of the experimental and numerical studies. Although the testing protocol in this thesis is similar, the focus is different in that (1) soft cohesive soil is used, instead of the commonly tested cohesionless soil, (2) SFSI interaction effects are studied 
instead of the displacement and rocking of the foundation, and (3), various embedment depths are tested, as opposed to only surface placement of the foundation model.

Hosseinzadeh and Nateghi (2003) conducted shake table testing on scale models of five-, 10-, 15-, and 20-story buildings located on relatively soft soils. The square mat foundation models, placed on the surface of the soft soil, were contained within a special container that is assumed to be a flexible barrel by the author. The container and model setup is shown in Fig. 3-2. The period lengthening ratios and foundation damping ratios of the models were analyzed both analytically and from the empirical shake table testing data, and the results are presented in Table 3-1. The experimental data were similar to the finite element analyses results, which were modeled by FLUSH. The first mode period and the damping ratio of the structures increased as a result of SFSI for all four models. This study used soft clay soil as its soil prototype; however, not enough information was provided in the literature. In this study, no indication was given as to the model structure, material properties, or similitude scaling factor used. In addition, the models were only placed on the surface of the clay soil, and no embedment experiments were conducted. As a result, it was difficult to quantify the true inertial interaction effects. On the contrary, hoping to provide better results, various embedment depths of the foundation model with different first mode periods were accounted for in this thesis. 

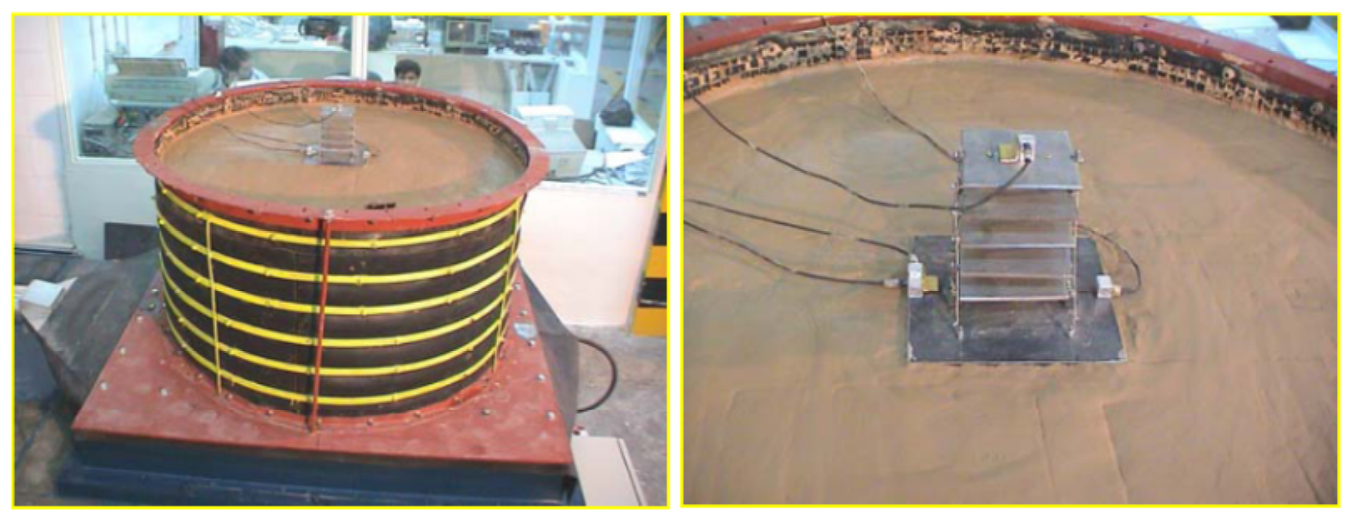

Figure 3-2 Setup of the shake table testing (from Hosseinzadeh and Nateghi 2003)

Table 3-1 The period lengthening ratio and the foundation damping ratio of the four models tested from the shake table testing (from Hosseinzadeh and Nateghi 2003)

\begin{tabular}{|c|c|c|c|c|c|}
\hline \multirow{2}{*}{$\begin{array}{c}\text { Building } \\
\text { type }\end{array}$} & \multicolumn{2}{|c|}{ Without SFSI } & \multicolumn{2}{c|}{ With SFSI } & \multirow{2}{*}{$(\bar{T} / T)$} \\
\cline { 2 - 5 } & $T(\mathrm{sec})$ & $\beta(\%)$ & $\bar{T}(\mathrm{sec})$ & $\bar{\beta}(\%)$ & \\
\hline 5 Story & 0.685 & 0.33 & 0.694 & 0.35 & 1.013 \\
\hline 10 Story & 1.287 & 3.40 & 1.299 & 3.50 & 1.010 \\
\hline 15 Story & 1.927 & 1.17 & 2.000 & 2.00 & 1.038 \\
\hline 20 Story & 2.597 & 1.30 & 2.786 & 2.10 & 1.073 \\
\hline
\end{tabular}

\subsubsection{Centrifuge Testing of Shallow foundation}

Shake table testing is a reliable, experimental protocol used to study the dynamic behavior of soil and structures. The behavior of the soil and structure holds true under $1 \mathrm{~g}$ full-scale conditions; however, the dynamic behavior can be considerably different in scale testing, especially since the gravitational force cannot be changed. Centrifuge model testing has become more common recently, due to its ability to better and more accurately mimic the gravitational force at different scale testing environments.

Rayhani and Naggar (2008) have conducted centrifuge testing of rigid foundation on soft soil. The tests were conducted at $80 \mathrm{~g}$ on a $5.5 \mathrm{~m}$ radius beam centrifuge. The 
centrifuge model configuration is shown in Fig. 3-3. The free-field and foundation level acceleration and displacements were recorded to evaluate the foundation response during dynamic excitation. T-bar tests (which are also used in this thesis and are presented in Chapter 4) were used to determine the shear strength of the model clay soil profile. The results of the centrifuge testing were used to benchmark the numerical model mentioned previously. The validated model was then used to study the effects of soil profile thickness and layering on seismic wave amplification.

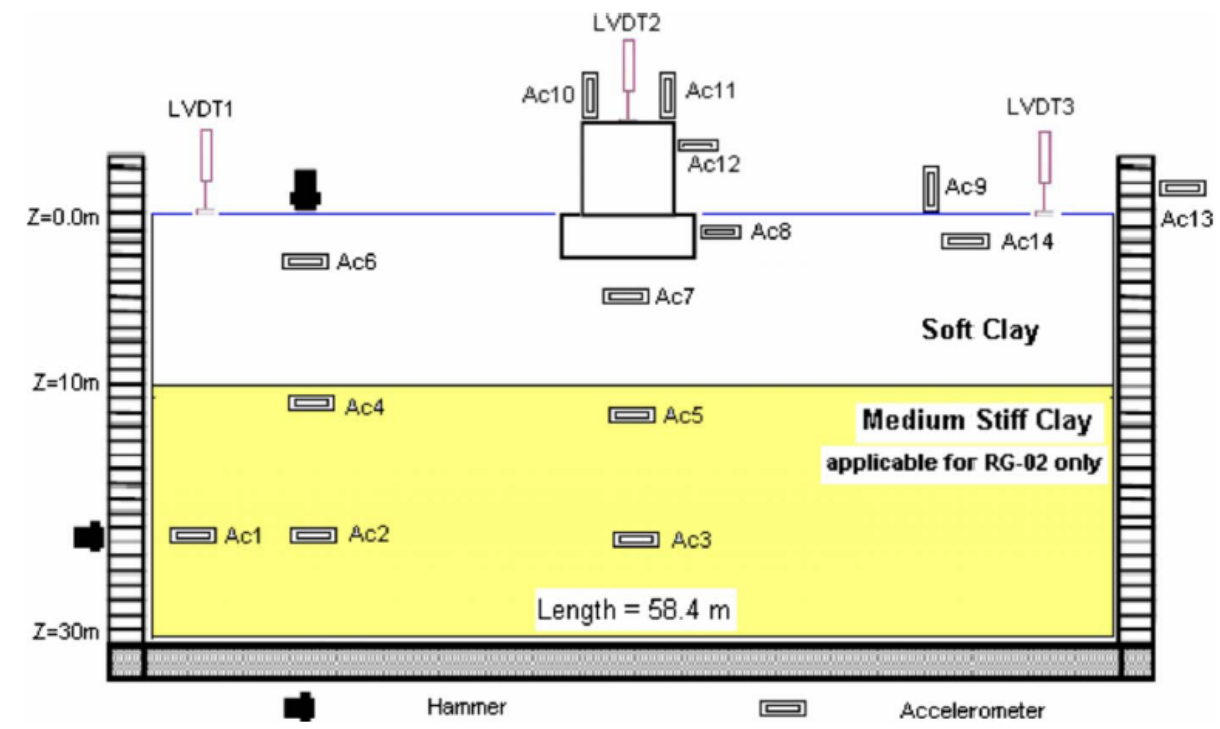

Figure 3-3 Setup of the centrifuge model and the placement of accelerometers and LVDTs (from Rayhani and Naggar 2008)

Pitilakis and Clouteau (2010) also used centrifuge SFSI tests to reference the aforementioned numerical model. A standard-degree-of-freedom (SDOF) model structure was placed on a bed of dry sand in the centrifuge container. The centrifuge setup of the model and data instrumentations is shown in Fig. 3-4. The tests were performed at $50 \mathrm{~g}$ environment, and the testing data were found to be similar to the numerical modeling results. However, no embedment effects were studied in either the empirical testing or numerical modeling. 


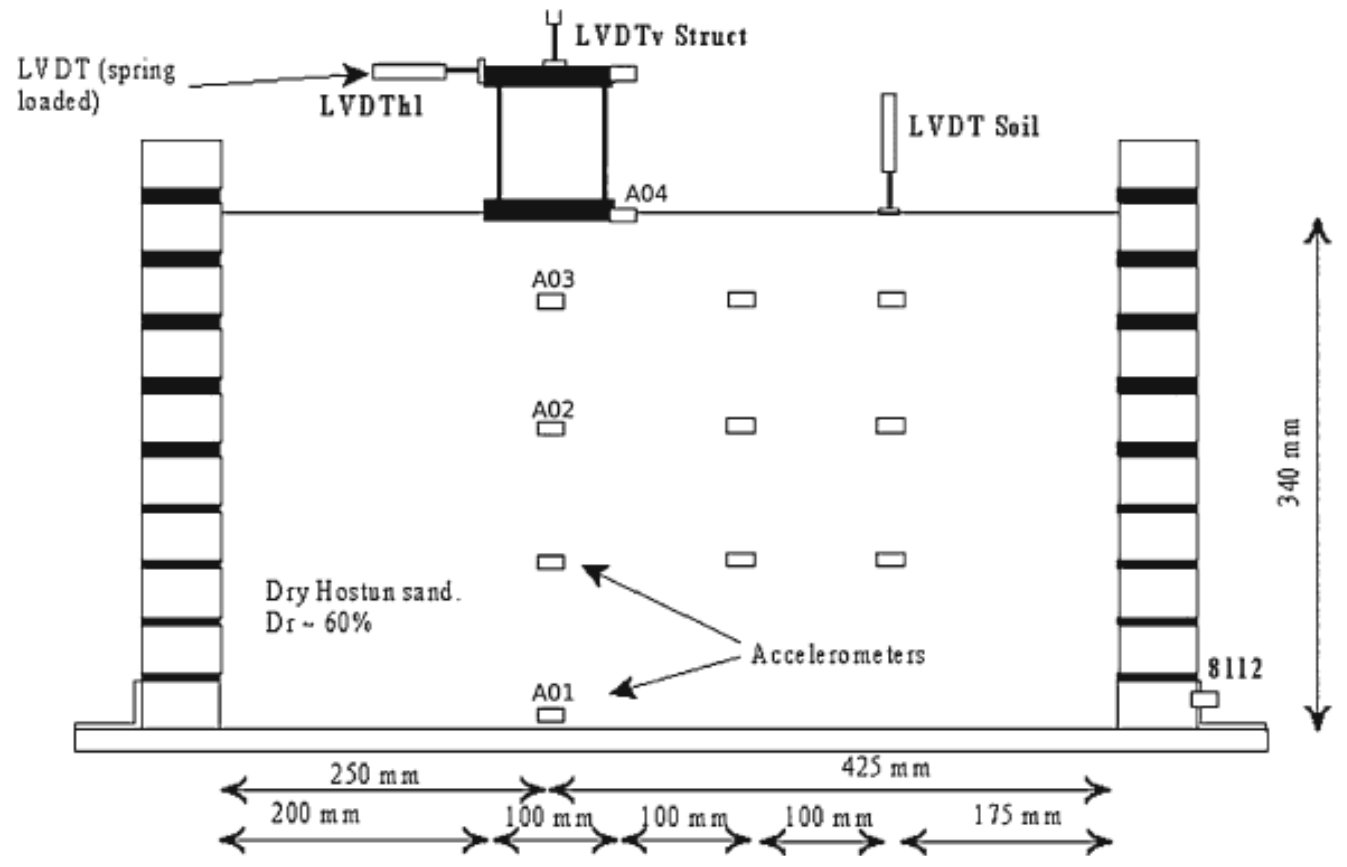

Figure 3-4 Setup and instrumentation of the centrifuge in the experiment (from Pitilakis and Clouteau 2010)

\subsection{Summary}

In this chapter, the theory and concept of soil-foundation-structure-interaction analysis were presented. The inertia and kinematic interactions were defined to provide additional background for the scope of the thesis. Kinematic interaction effects do not influence the overall dynamic structure responses as much as inertia interaction effects for shallow foundations in soft clay. The focus of this thesis is on the inertia interaction effects.

Two types of SFSI analyses are the direct and substructure methods. The direct method accounts for nonlinear soil behavior and analyzes the system in one step, often in finite element analyses; however, the computation time and costs are rather high. The substructure method divides the system into different domains, and due to its simplicity and efficiency, it has been incorporated into the design codes. The SFSI effects, primarily 
those from inertia interaction, can be quantified by the period lengthening ratio and the foundation damping ratio of the fixed- and flexible-base scenarios. There are many existing analytical formulations and numerical modeling techniques to analyze the influence of SFSI.

Few empirical 1-D shake table SFSI studies have been performed in the past (Shirato et al. 2008; Hosseinzadeh and Nateghi 2003) and are presented previously to demonstrate the effectiveness of experimental evaluation of SFSI. Prior shake table studies for geotechnical applications were mostly conducted with sandy soils (Iai 1999), thus explaining the lack of empirical studies involving soft clay soils. Recently, centrifuge testing has become more popular because of its ability to simulate scale dynamic responses when gravitational loads are vital to soil strength. However in this thesis, shake table study is the choice testing apparatus because of its focus upon the SFSI effects of shallow foundations on soft clay soils, where soil strength is stress independent. 


\section{CHAPTER 4 - DEVELOPMENT OF TESTING PLATFORM}

\subsection{Introduction}

Using a controlled lab environment shake table testing platform, different factors that contribute to SSI effects on the building seismic response, such as foundation embedment depth and building fundamental period, can be tested individually. Specific analysis of how each parameter influences the overall building response is difficult due to the complex interaction and size of all the different components. Scale model testing results can also serve as a calibration benchmark for further analytical studies for predicting prototype response (Meymand 1998). This study utilizes scale model shake table testing to analyze the influence of foundation embedment on building dynamic behavior in soft clay. Scale model testing provides an economical and easily repeatable means of collecting data, as opposed to the expensive and difficult-to-simulate full scale testing.

As mentioned in Chapter 1, the testing platform used in this study was originally developed by Meymand (1998), then modified and tested by Crosariol (2010). The comprehensive review of scale model similitude laws and testing platform development are described in those studies. This chapter serves as an executive summary on the works of Meymand and Crosariol, and then focuses on scale model development and testing methods unique to this study. The detailed testing program is outlined in Chapter 4.

\subsection{Scale Model Similitude}

In order to simulate the dynamic behavior of a full scale system, similitude theory relating the testing model to the prototype must be applied. Langhaar (1951) indicates 
that scale models have geometric, kinematic, and dynamic similarities to the prototype. According to Meymand (1998), geometric similarities link a model and prototype with physical dimensions, kinematic similarities define a model and prototype with corresponding materials at corresponding locations at corresponding times, and dynamic similarities refer to a model and prototype experiencing similar forces at the corresponding locations. Scale models have varying degrees of compliance with the similitude to the prototype. Moncarz and Krawinkler (1981) categorize scale modeling proficiency into true, adequate, and distorted models. Each of these similitude relationships must be fulfilled for a true model. An adequate model properly scales the primary factors, but allows for secondary parameters to deviate. A distorted model does not accurately predict true prototype response unless compensating distortions are introduced to the prediction equations or physical parameters.

Kline (1965) defines dimensional analysis, similitude theory, and governing equations as three methods used for scaling. First, dimensional analysis converts equivalent dimensional equations based on the fundamental Mass-Length-Time "measures of nature" while using a scale factor for these quantities. Second, similitude theory utilizes dimensional analysis and incorporates dimensionless terms by identifying equivalent forces acting on the system. Lastly, the method of governing equations uses differential equations to transform the system into non-dimensional form, and it is the most complex and powerful method.

Dimensional analysis method simplifies engineering parameters to fundamental mass $(\mu)$, length $(\lambda)$, and time $(\tau)$ "measure of nature" to correctly scale relations between model and prototype. Sample dimensional equation derivations are presented in 
Meymand (1998) and Crosariol (2010) using a geometric scaling factor $(\lambda)$, which is the ratio of prototype to model. With that concept, the complete set of parameters pertinent to the study can be derived using the correct dimensional scaling relations.

Meymand (1998) reviews the Buckingham Pi Theory, which states that "any dimensionally homogeneous equation involving certain physical quantities can be reduced to an equivalent equation involving a complete set of dimensionless products." The Pi term, an independent dimensionless product of the physical qualities, is applied to group the physical variables into a dimensionless term. Essentially, Buckingham Pi Theorem is a more complex dimensional analysis method. Kokusho and Iwatate (1979) used this theorem to develop the similitude of nonlinear dynamic responses. However, Iai (1989) claims the results are only applicable to shear deformation of soil structures, but not the overall dynamic behaviors for saturated fluid-soil-structure condition.

Rocha (1975) first presented the scale modeling issue in soil mechanics. He derived similitude relations for different soil stress states and concluded that if the stress and strain are held in a linear relationship between the model and prototype, then the soil constitutive behavior can be scaled under a 1-g condition. However, the derivation only held true for elastic deformation.

Iai (1989) developed a similitude scaling relation for shake table tests on saturated soil-structure-fluid scale model in 1-g gravitational field under dynamic loading. The similitude was derived using basic equations, such as equilibrium and mass balance of soil skeleton, pore water, and constitutive law of soil, that govern the behavior of saturated soil-structure-fluid systems under seismic loading. Readers can find further 
information in Iai (1989) and Crosariol (2010) detailing the similitude derivations. A summary of similitude criteria relevant for this study is presented in Table 3-1.

Moncarz and Krawinkler (1981) built on the Buckingham Pi Theorem and claimed two conditions must be fulfilled for "true" scale modeling: the Froude number and Cauchy condition. In 1-g scale modeling, Froude's number is a dimensionless number defined, in this case, as the ratio of prototype to model shear wave velocity. Cauchy condition indicates that the Froude number, which can also be presented as the ratio of model to prototype specific stiffness in terms of material modulus (E) and density ( $\rho$ ), should be equal to the geometric scaling factor $(\lambda)$. Moncarz and Krawinkler also stressed that the Cauchy condition is a critical requirement for concurrently scaling pertinent forces in a dynamic model system. For further theoretical justification and equation derivation, please refer to Moncarz and Krawinkler (1981).

Dynamic model testing in 1-g condition is plausible by using scaling similitude relations established by Iai and obeying the conditions of scale model testing developed by Moncarz and Krawinkler. Particular scale model replication of the prototype may be difficult due to the natural variation in building materials, but adjustments may be made to achieve an adequate scale model. A distributed or lumped mass can be added to the model for suitable seismic behavior without significantly changing the structural layout (Meymand 1998).

\subsubsection{Scale Modeling Similitude for Shake Table Testing}

The appropriate interaction modes of SFSI system response were presented in Chapter 1. These are free-field soil site response, soil-foundation kinematic interaction, 
soil-foundation inertial interaction, physical interaction, and damping. Table 4-1 lists the parameters associated for each of the SFSI modes.

Table 4-1 SFSI interaction modes and relevant variables (adopted from Meymand, 1998; Crosariol, 2010)

\begin{tabular}{|c|c|}
\hline SFSI Interaction Mode & Variables \\
\hline 1. $\quad$ Free-field Site Response & $\begin{array}{l}\text { Shear wave velocity }\left(\mathrm{V}_{\mathrm{s}}\right) \\
\text { Soil density }\left(\rho_{\mathrm{s}}\right) \\
\text { Modulus degradation (G/Gmax) } \\
\text { Damping }(\beta)\end{array}$ \\
\hline 2. Kinematic Interaction & $\begin{array}{l}\text { Free-field site response } \\
\text { Flexural rigidity (EI) } \\
\text { Structural geometry }(\mathrm{L})\end{array}$ \\
\hline 3. Inertial Interaction & $\begin{array}{l}\text { Stiffness }(\mathrm{K}) \\
\text { Structural mass }(M) \\
\text { Flexural rigidity }(\mathrm{EI}) \\
\text { Structural geometry }(\mathrm{L}) \\
\end{array}$ \\
\hline 4. Physical Interaction & $\begin{array}{l}\text { Construction } \\
\text { Dynamic loading (F) }\end{array}$ \\
\hline 5. Damping & $\begin{array}{l}\text { Free-field site response } \\
\text { Material modulus (E) } \\
\text { Structural mass (M) } \\
\text { Structural geometry (L) } \\
\end{array}$ \\
\hline
\end{tabular}

Most of these variables can be properly scaled using the geometric scaling factor with similitude relations developed by Iai. Material mass (M) can be difficult to scale properly for scale models since radiation damping is dependent on the overall mass (Meymand 1998). However, Meymand notes that at high frequency levels, which often occur during shake table testing, radiation damping should have little effect on the overall response. Physical interactions, such as those caused by construction, cannot be accurately modeled during scale model construction. Since the degree of influence cannot be measured, physical interaction of construction is disregarded.

Based on the previously mentioned similitude scaling criteria for scale model testing on a 1-g shake table, a list of pertinent scaling relations for this study is presented 
in Table 4-2. Given the limitations of the testing equipment, the geometric scaling factor ( $\lambda$ ) of 10 was selected for this research based on the discussion in Crosariol (2010). The scale factor can be substituted with different numbers and the similitude criteria holds true.

Table 4-2 Scale factors for pertinent engineering variables in terms of geometric scaling factors ( $\lambda$ ) (adapted from Iai, 1989; Meymand, 1998; Crosariol, 2010)

\begin{tabular}{|l|c|c|}
\hline Variable & Scale Factor & For $\lambda=10$ \\
\hline Soil Density & 1 & 1 \\
\hline Force & $\lambda^{3}$ & 1000 \\
\hline Stiffness & $\lambda^{2}$ & 100 \\
\hline Modulus & $\lambda$ & 10 \\
\hline Acceleration & 1 & 1 \\
\hline Shear wave Velocity & $\lambda^{1 / 2}$ & 3.16 \\
\hline Soil Damping & 1 & 1 \\
\hline Poisson's ratio & 1 & 1 \\
\hline Time & $\lambda^{1 / 2}$ & 3.16 \\
\hline Frequency & $\lambda^{-1 / 2}$ & 0.316 \\
\hline Length & $\lambda$ & 10 \\
\hline Stress & $\lambda$ & 10 \\
\hline Strain & 1 & 1 \\
\hline Flexural Rigidity & $\lambda^{5}$ & 100000 \\
\hline Dimensionless Quantities & 1 & 1 \\
\hline
\end{tabular}

\subsection{Development of Model Soil}

The model soil used in this study was created by Crosariol (2010) modeling after Meymand's (1998) research for SSPSI effects in soft clay. Scale model testing for SFSI of soft cohesive clay soil is uncommon. The model soft clay soil was designed by following the similitude scaling criteria for both the free-field and soil-foundationinteraction conditions, which is appropriate for this study. The five main non-linear soil parameters pertinent to scale model soil are density, modulus reduction and damping, stress-strain response, shear wave velocity, and undrained shear strength (Meymand 
1998). Meymand detailed the iteration process to develop the model soil mix given the implied prototype soil properties in his research.

San Francisco Young Bay Mud serves as the prototype soil for this study (Meymand 1998). Model soil used in this study is created by mixing $67.5 \%$ kaolinite, $22.5 \%$ bentonite, and $10 \%$ class $\mathrm{C}$ fly ash with a target water content of $125 \%$. Table $4-3$ compares the soil properties between the prototype and model soil mix. The ingredients purchased for Crosariol's model soil at Cal Poly were as close as those used by Meymand. Crosariol outlines the ingredients as: the bentonite is American Standard 200 mesh, distributed by Scott Sales Company in Huntington Park, CA; the Kaolinite is Kamin 35 (formerly Huber 35), distributed by the PT Hutchins 68 Company in City of Industry, CA; and the class $\mathrm{C}$ fly ash was obtained through Mineral Resources Technologies, a subsidiary of Cemex USA (Crosariol 2010). Detailed explanations of the origin and development for this specific model soil "recipe" can be found in Meymand and Crosariol's studies.

Table 4-3 Prototype soil properties and the model soil mix for this study (after Meymand 1998; Crosariol 2010)

\begin{tabular}{|l|l|l|}
\hline Property & Bay Mud & Model Soil \\
\hline Saturated Unit Weight $\left(\mathrm{kN} / \mathrm{m}^{3}\right)$ & 14.8 & 14.8 \\
\hline Water Content (\%) & 90.00 & 100.0 \\
\hline Liquid Limit (\%) & 88.00 & 115.00 \\
\hline Plastic Limit (\%) & 48.00 & 40.00 \\
\hline Plasticity Index (\%) & 40.00 & 75.00 \\
\hline Coefficient of Consolidation Cv $\left(\mathrm{m}^{2} /\right.$ year) & 0.75 to 0.92 & $6.5 \times 10^{-3}$ \\
\hline Undrained Shear Strength $(\mathrm{kPa})$ & 29 to 57 & 4.1 \\
\hline Shear wave Velocity $(\mathrm{m} / \mathrm{s})$ & 114 to 160 & 40.0 \\
\hline
\end{tabular}




\subsection{Selection of Input Motions}

There are 7 motions selected as input motions for this shake table testing program. A summary of these ground motions is listed in Table 4-4.

Table 4-4 List of motions used for this study and earthquake information

\begin{tabular}{|c|l|l|c|c|c|}
\hline No. & \multicolumn{1}{|c|}{ Earthquake } & \multicolumn{1}{|c|}{ Station Name } & Prefix & Date & Mag. \\
\hline \hline 1 & Landers & 22170 Joshua Tree & JOS & $6 / 28 / 1992$ & 7.3 \\
\hline 2 & Imperial Valley & 117 El Centro Array \#9 & ELC & $5 / 19 / 1940$ & 6.9 \\
\hline 3 & Chi Chi & TCU75 & TCU & $9 / 20 / 1999$ & 7.6 \\
\hline 4 & Imperial Valley & Supersition Mtn. Camera & IPV & $10 / 15 / 1979$ & 6.5 \\
\hline 5 & Loma Prieta & Los Gatos Presentation C. & LGP & $10 / 18 / 1989$ & 6.9 \\
\hline 6 & Cape Mendocino & Cape Mendocino & CPM & $4 / 25 / 1992$ & 7.1 \\
\hline 7 & Northridge & Lake Hughes \#9 & LO9 & $1 / 17 / 1994$ & 6.7 \\
\hline
\end{tabular}

The first 3 ground motions (Joshua Tree, El Centro, and TCU) have been previously selected and tested in the shake table testing program here at Cal Poly by Crosariol (2010). Therefore, these three motions are selected again for this study to provide comparable testing data and performance validation. Four additional ground motions are selected to provide an adequate frequency range of peak spectral acceleration for the testing program. These fours motions (Supersition Mtn, Los Gatos, Cape Mendocino, and Lake Hughes) are picked from an extensive study by Bazzurro and Cornell (2004) on ground motion amplification in nonlinear soils.

In order to fit the motions to model scale, the time steps of the ground motions are scaled by $\Delta \mathrm{t} / \lambda^{0.5}$ to obey the aforementioned similitude scaling relation. The geometric scaling factor for this study has been established as 10 to allow data comparison to Crosariol's study. With $\lambda=10$, the time step for the motions have to be compressed by a factor of 3.16. 
For each of the motions, both horizontal azimuths are included in the testing program; therefore, there are 14 total ground motions used in this study. Figure 4-1 shows different amplitudes and frequencies of the peak spectral acceleration of all the input ground motions. The individual scaled input ground motions are shown in the following figures.

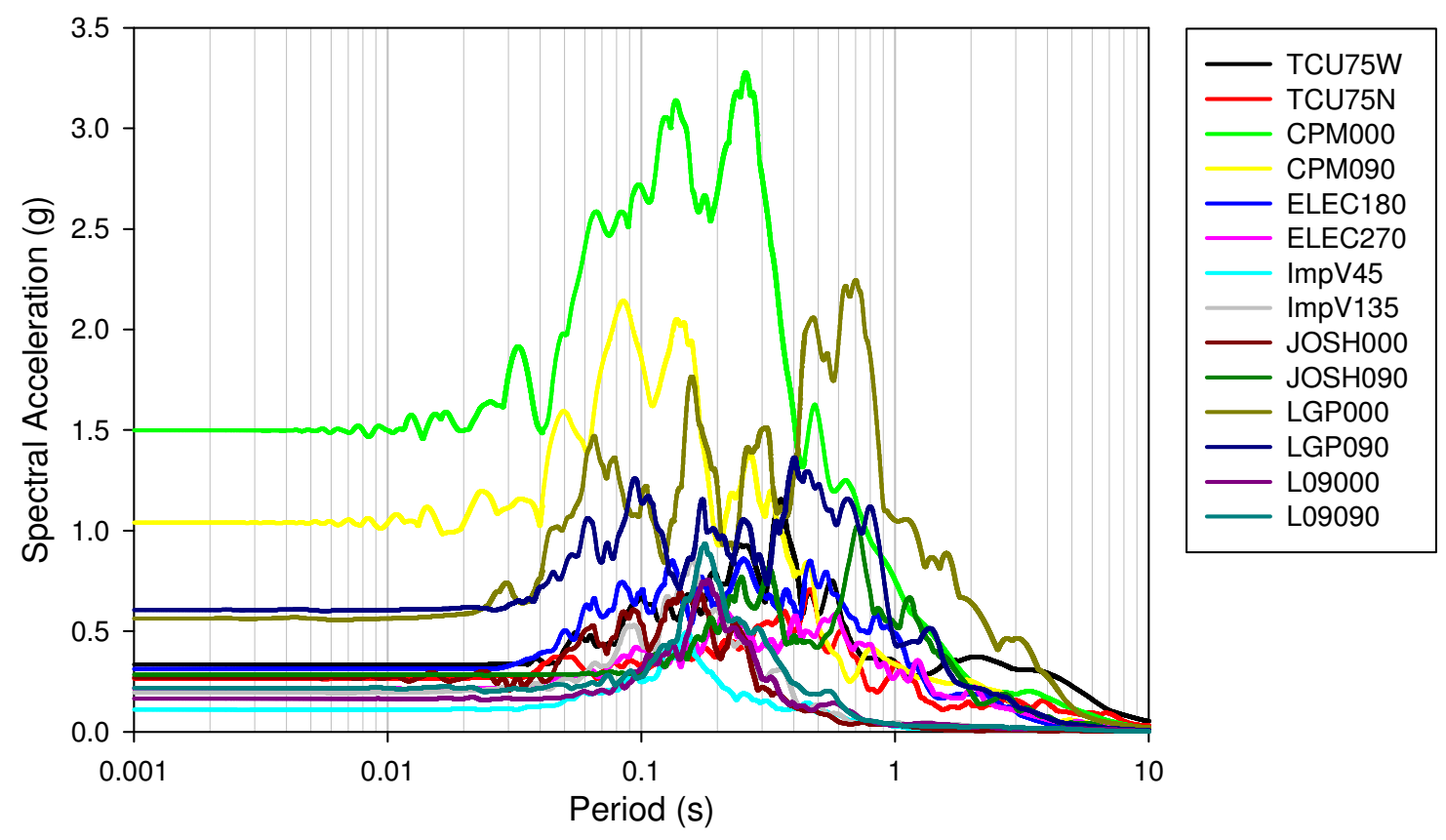

Figure 4-1 Spectral acceleration summary of the 14 motions used in this study. These motions cover a range of various peak spectral accelerations and corresponding periods.
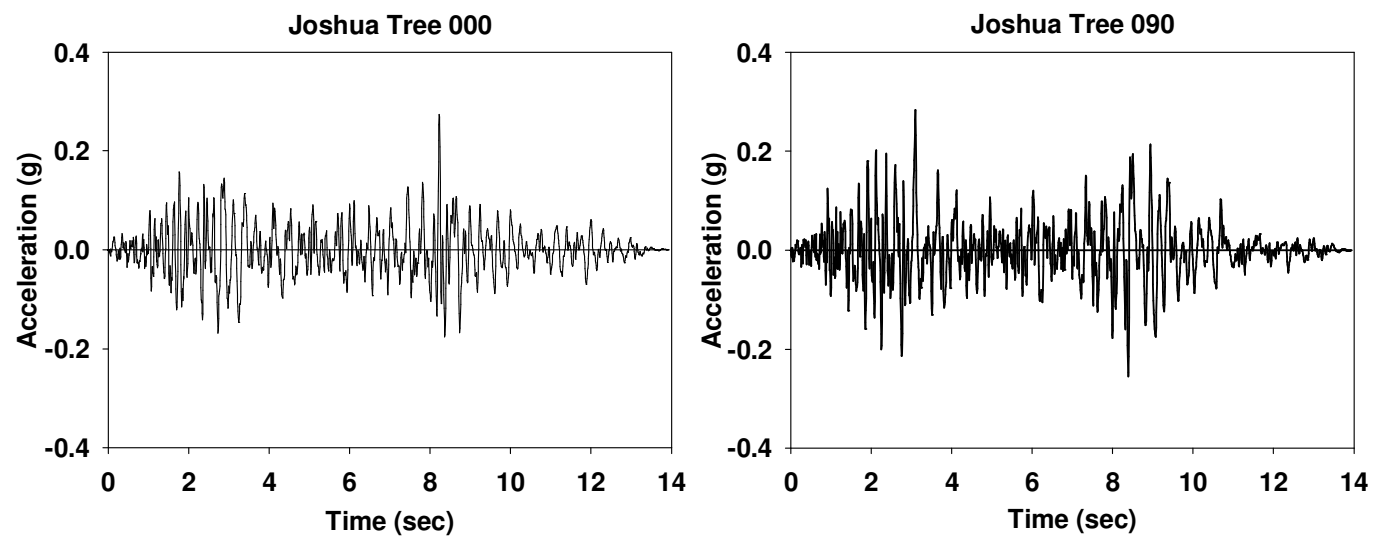

Figure 4-2 Scaled Joshua Tree motions for both horizontal azimuths of the 1992 Landers earthquake with compressed time step by $\lambda^{0.5}$ 

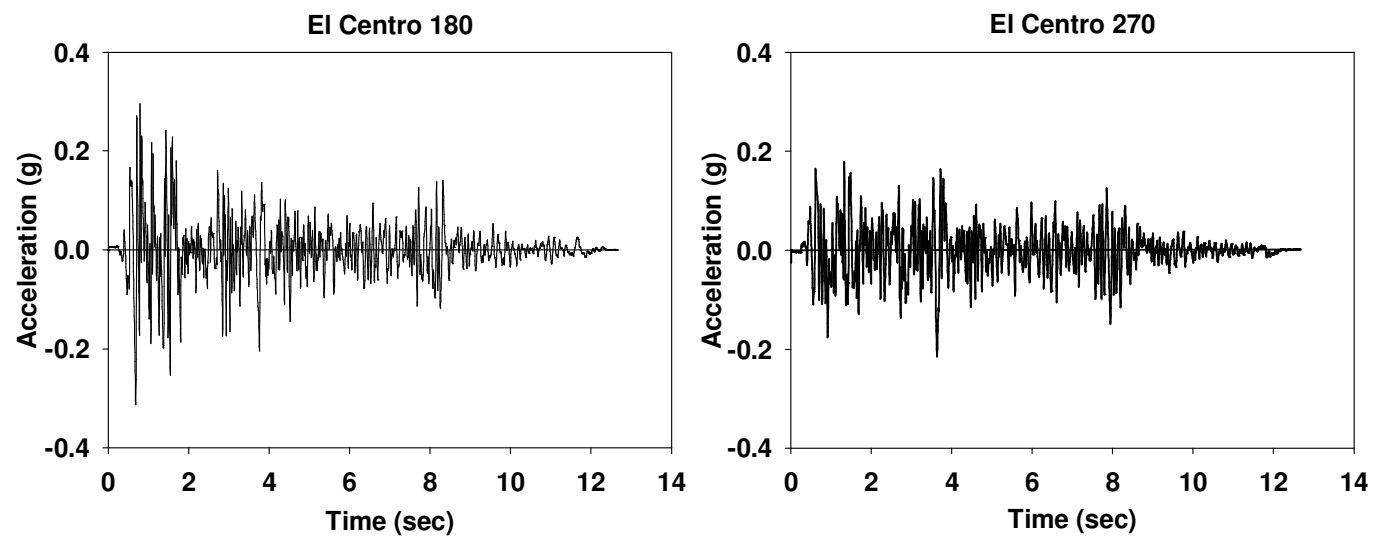

Figure 4-3 Scaled El Centro motions for both horizontal azimuths of the 1940 Imperial Valley earthquake with compressed time step by $\lambda^{0.5}$
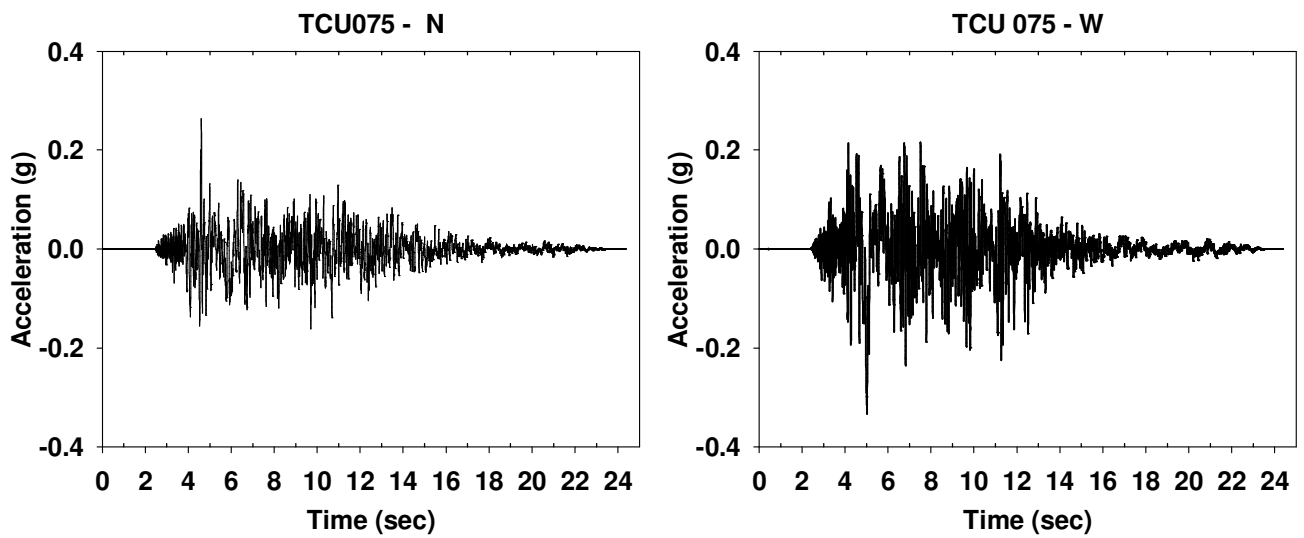

Figure 4-4 Scaled TCU075 motions for both horizontal azimuths of the 1999 Chi Chi earthquake with compressed time step by $\lambda^{0.5}$
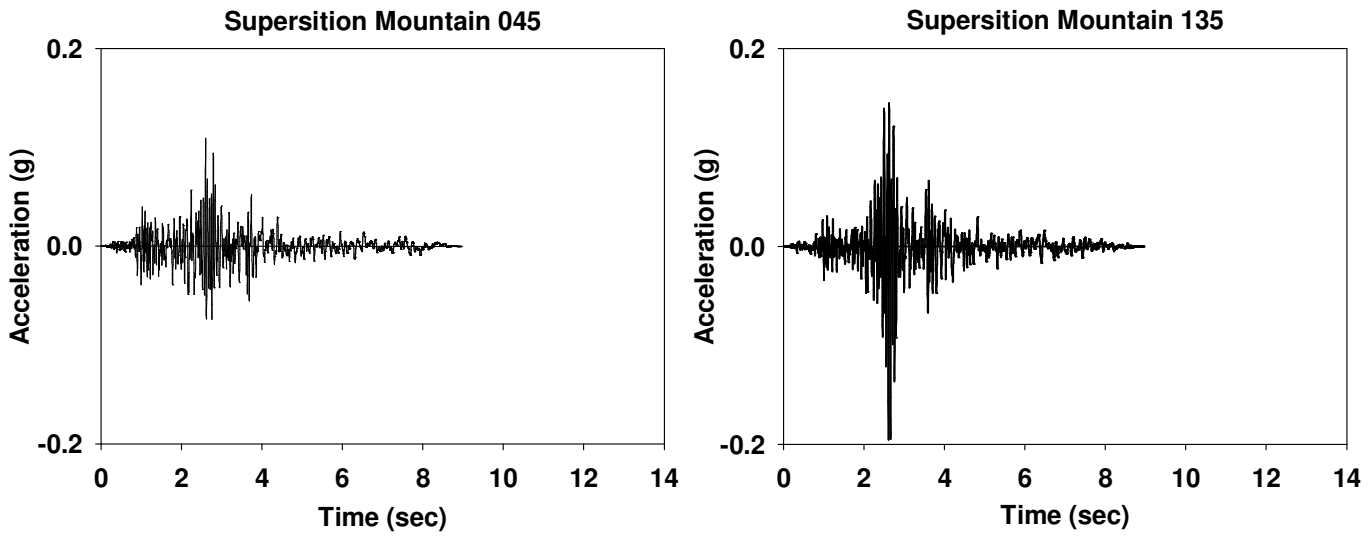

Figure 4-5 Scaled Supersition Mountain motions for both horizontal azimuths of the 1979 Imperial Valley earthquake with compressed time step by $\lambda^{0.5}$ 

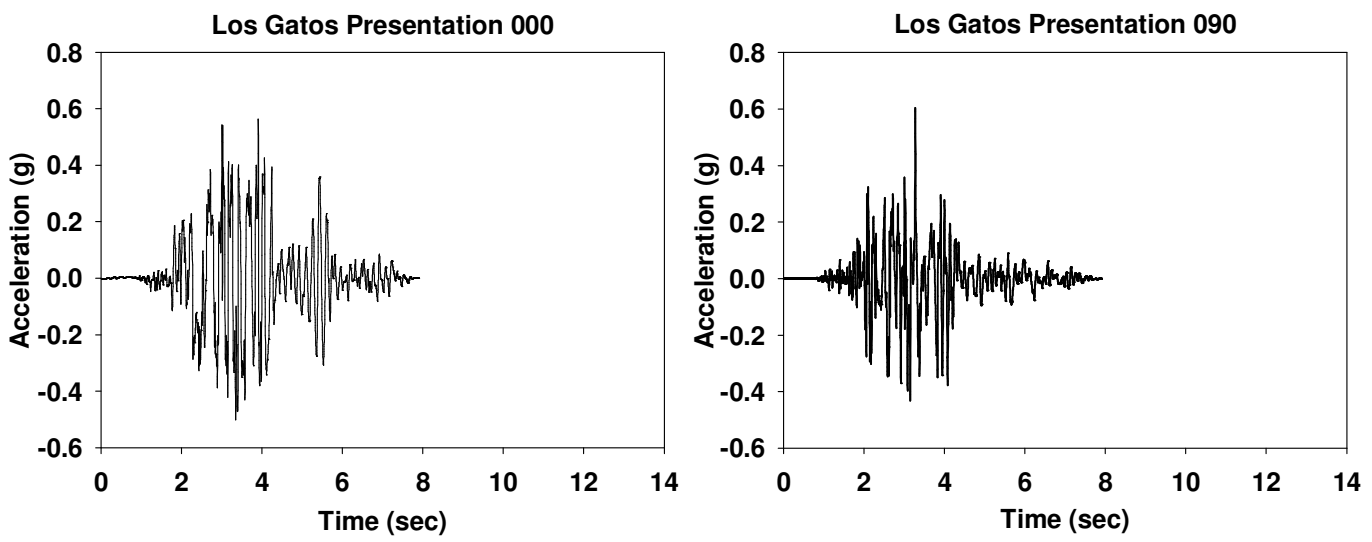

Figure 4-6 Scaled Los Gatos motions for both horizontal azimuths of the 1989 Loma Prieta earthquake with compressed time step by $\lambda^{0.5}$
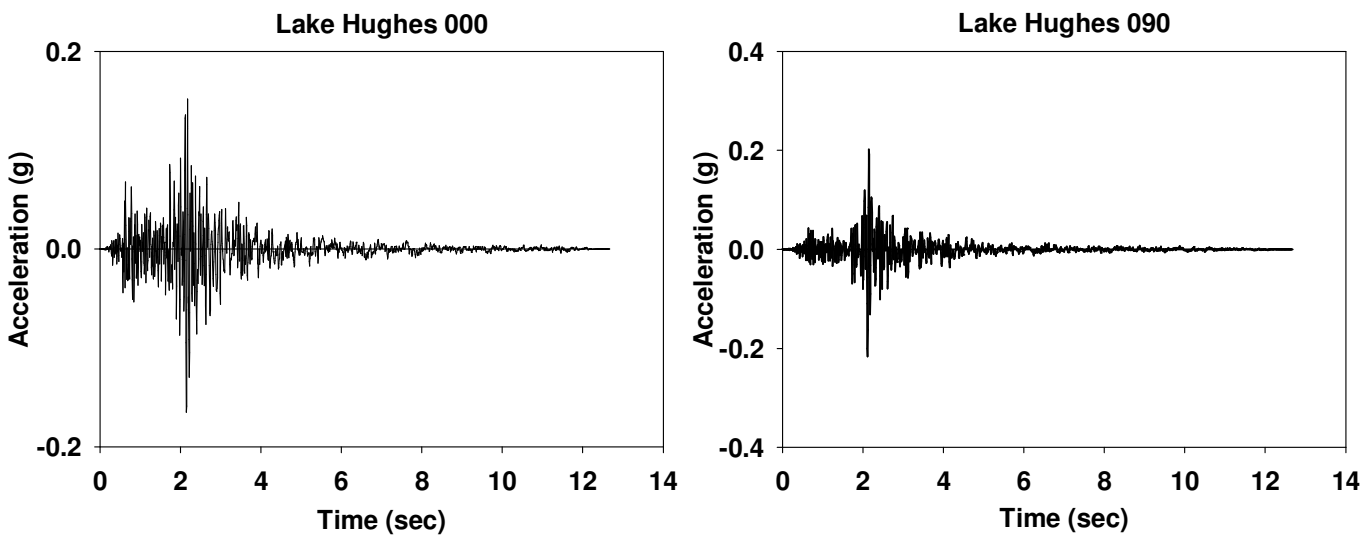

Figure 4-7 Scaled Lake Hughes motions for both horizontal azimuths of the 1994 Northridge earthquake with compressed time step by $\lambda^{0.5}$ 

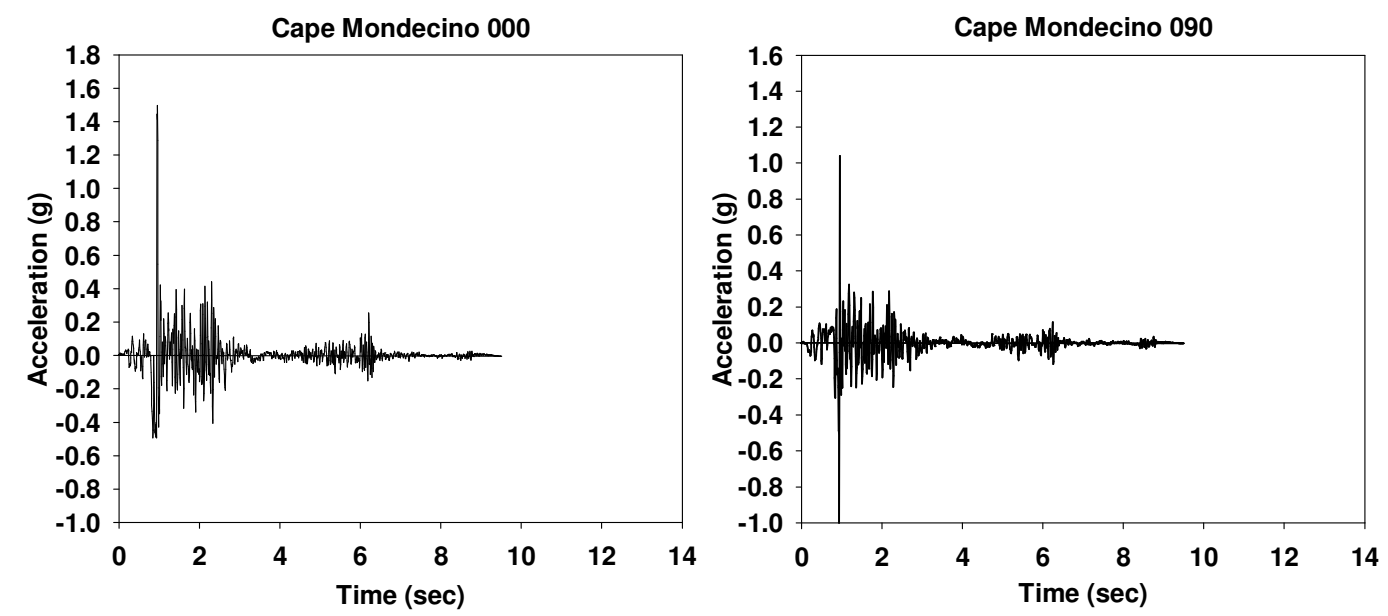

Figure 4-8 Scaled Cape Mondecino motions for both horizontal azimuths of the 1992 Cape Mondecino earthquake with compressed time step by $\lambda^{0.5}$

\subsection{Development of Scale Model}

One of the main goals of this research is to obtain laboratory controlled empirical testing results on inertial SSI effects in buildings in addition to the field observation collected by Stewart et al. (1999b). Stewart et al. (1999b) selected 57 sites with strong motion recordings to interpret the effects of inertial SSI on seismic structure response. Stewart et al. (1998) concluded that the following factors have greatest influence on inertial interaction effects: ratio of structure-to-soil stiffness, structure aspect ratio, foundation embedment, foundation type, foundation shape effects, and foundation flexibility effects.

The target prototype building of the scale model was developed after reviewing the 57 sites. With inertial interaction effects as the primary interest, the structure-to-soil stiffness ratio has to be notable (Stewart et al. 1998). Since a model soft clay soil is used, a significant structure-to-soil stiffness ratio is present with the use of rigid building material. Deeper foundation embedment increases damping as a result of dynamic soilbasement-wall interaction for an embedment ratio of over 0.5 (Stewart et al. 1998). 
Embedment ratio (e/r) is the ratio between the embedment depth (e) and the equivalent radius of the foundation area (r). The target embedment ratios for the scale model are 0.5 and 1.0 because the model basement wall must be tall enough to meet the desired embedment depth.

For the scale model development, geometric constraints due to testing container size and boundary effects induced from the rubber sidewall during dynamic loading put a limitation on the foundation size. Crosariol (2010) states that near the soil surface, maximum spectral acceleration near the testing container sidewall is $10 \%$ higher than the center of the soil column. With the intention of having both free-field and model array present simultaneously on the soil column surface, the scale model has to be placed relatively far from the sidewall to avoid boundary effects. In addition, the scale model must be far away to not influence the free-field acceleration data. As a result, limited space is available for the scale model foundation.

With the above design constraints, the proposed prototype building has a square mat foundation. The fundamental period of the prototype building is set to be around 1.0 and 2.0 after investigating the 57 sites studied in Stewart et al. (1998) as this experiment plans to expand data on tall building seismic response. Further design and material selection details of the scale model are presented in the following section.

\subsubsection{Design Configuration and Material}

The design configuration and materials selected for the scale model are based on a damped single degree-of-freedom system (SDOF) structure that has variable fundamental period and embedment depth. The shallow foundation structure also has to be designed 
against overturning, especially during dynamic loading. Settlement is not a concern in this case since the clay soil is maintained in an undrained state.

Given the target fundamental period of the prototype building and the scale factor, the configuration of the lump-mass "lollipop" setup is back calculated using the free vibration equations of a SDOF system. The stiffness (k) of the SDOF structure is calculated using equation 3.1.

$$
k=\frac{3 E I}{h^{3}}
$$

Where: $\mathrm{E}=$ Young's modulus; $\mathrm{I}=$ Second moment of area; $\mathrm{h}=$ effective height of the lumped-mass. Once the stiffness of the overall system is determined, the natural circular frequency $\left(\omega_{n}\right)$ is calculated using equation 3.2.

$$
\omega_{n}=\sqrt{\frac{k}{m}}
$$

Where: $\mathrm{m}=$ mass of the lumped-mass. The natural period of vibration $\left(T_{n}\right)$ of the system is related to the natural circular frequency equation 3.3.

$$
T_{n}=\frac{2 \pi}{\omega_{n}}
$$

Under free vibration, all real systems depict some decay in their response over time due to energy dissipation or damping. The damping behavior is caused by various energy losses in the system, such as friction, resistance of the medium, or inelasticity of the material (Chopra 2001). Damping is a complex variable that is difficult to quantify and is usually approximated through equivalent viscous damping. For this particular model, it is assumed to be underdamped, where the range for the damping ratio is 
typically between $2 \%$ and $20 \%$. The damped natural period of vibration $\left(T_{D}\right)$ of the system can be quantified with equation 3.4.

$$
T_{D}=\frac{T_{n}}{\sqrt{1-\xi^{2}}}
$$

Where: $\xi=$ damping ratio. For simplicity during the design process, the SDOF model damping was assumed to be $5 \%$. Using these fundamental vibration equations, the optimal mass, height, and materials were selected based on the initial calculation.

Once the appropriate height and mass of the lollipop model were selected, an overturning analysis was executed for seismic loading. The peak ground acceleration is assumed to be $2.0 \mathrm{~g}$ on the lumped mass. As a result, a heavy foundation is required to prevent the model from overturning when experiencing extreme horizontal acceleration during shake table testing. A square steel plate of $45.7 \mathrm{~cm}$ wide by $45.7 \mathrm{~cm}$ wide and 1.8 $\mathrm{cm}$ in thickness was used. Due to the highly corrosive condition provided by the fly ash content in the model soil, the steel plate is coated with layers of rust resistance paint. The stiffness of the steel base also provides a significant structure to soil stiffness ratio that may contribute to inertial interaction effects.

The embedment depth is approximated based on desired embedment ratios and the selected foundation area. With the selected foundation, a $30 \mathrm{~cm}$ wall height was selected to allow embedment ratios of less than 0.5 , between 0.5 and 1.0 , and over 1.0. The structural frame of the basement was made out of $2.54 \mathrm{~cm}$ wide L-shaped aluminum beams and plates that were reinforced with screws and rivets. Walls were built on all sides, creating an embedded basement by using rust resisting acrylic boards. The exposed screws were covered with silicon caulking to resist rust as well. 
The SDOF lumped mass is attached to a $1.9 \mathrm{~cm}$ diameter threaded carbon steel (A307) rod. The initial weight and height selection is approximated analytically using the equations provided in this section. A parametric study was conducted on the actual shake table to determine the exact setup. The lumped mass weighs $9.1 \mathrm{~kg}$ and is secured on the threaded rod with washers and hex nuts, so the position of the lumped mass is adjustable to achieve different desired fundamental periods. Two different height positions at $52 \mathrm{~cm}$ and $72 \mathrm{~cm}$ from the top of the foundation plate to the center of the lumped mass serve as the final setup. The process of locating these heights is further explained in Chapter 5 for the small shake table testing results and analysis.

A rail guide device was employed to ensure the lollipop moved in one direction during dynamic loading. Any deviation from the shaking direction results in additional energy dissipation and non-measurable error in the result. Two $6.4 \mathrm{~cm}$ wide blocks of acrylic with slots for two stainless steel rods to run parallel to the shaking direction are located on either side. The stainless steel rail guides are flushed against a pre-installed coupling nut on the threaded rod and are lubricated to reduce contact friction. Zip-ties are used to secure the rail guides in place. These rail guides can be removed in between the two model height setups. Figure 4-9 provides a view of the rail guide setup. An overview of the scale model design is presented in Figure 4-10. 


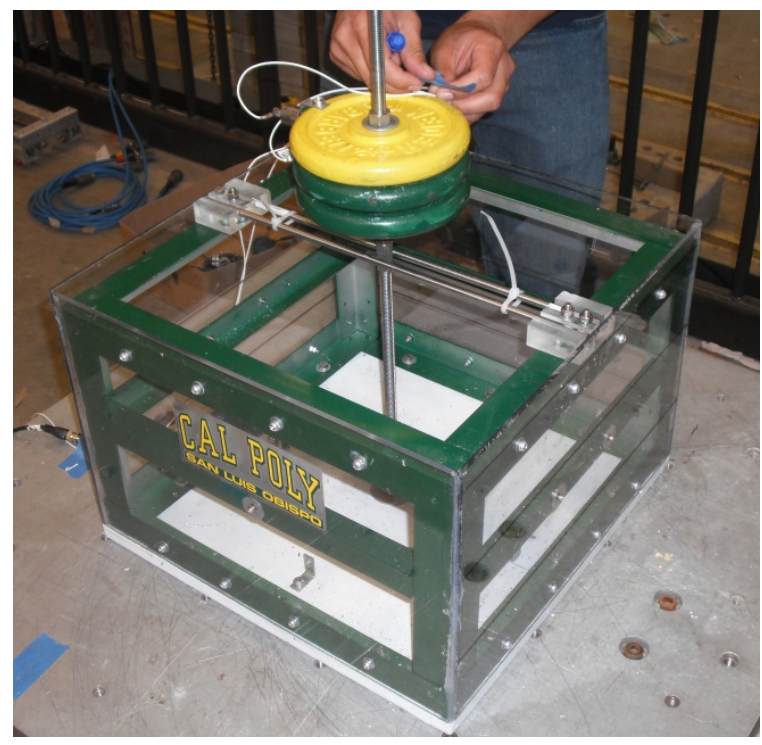

Figure 4-9 An angle snapshot of the final scale model. The model is secured to the small shaking table, which is introduced in the next chapter. Note the accelerometer attached to the weight plate for vibration data collection

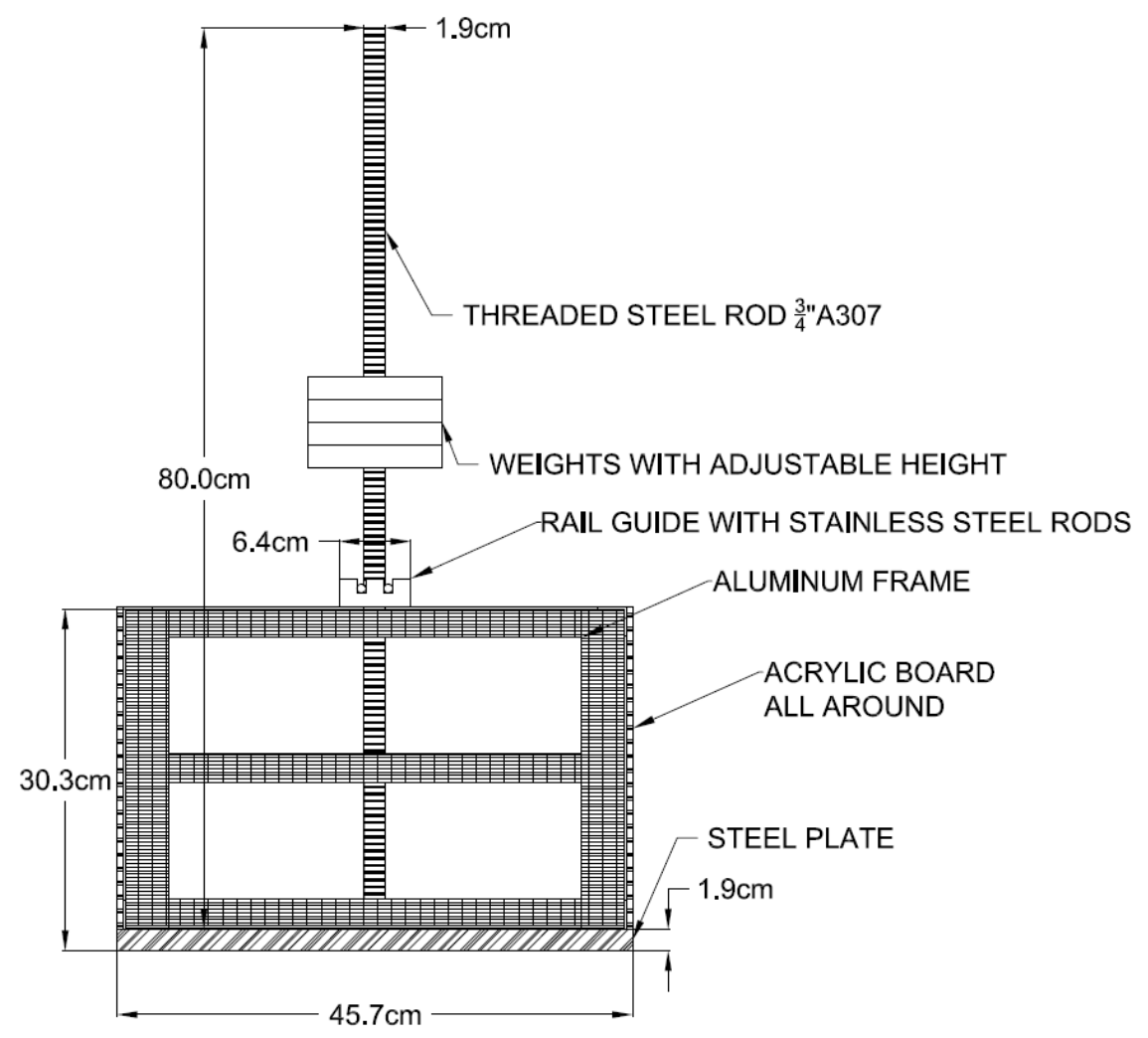

Figure 4-10 Scale model design and materials used 


\subsection{Model Testing Container}

The main testing equipment is a flexible wall barrel that mimics free field site response under seismic loading on the shake table. The flexible wall barrel has been validated through table testing and $1 \mathrm{D}$ equivalent linear numerical analysis in a previous research project (Crosariol, 2010; Moss et al., 2010). Figures 4-11 and 4-12 demonstrate the dynamic performance of the flexible wall barrel compared to the other testing platforms. The prototype soil column has a seismic response that is most similar to the result from the flexible wall barrel (Meymand 1998).

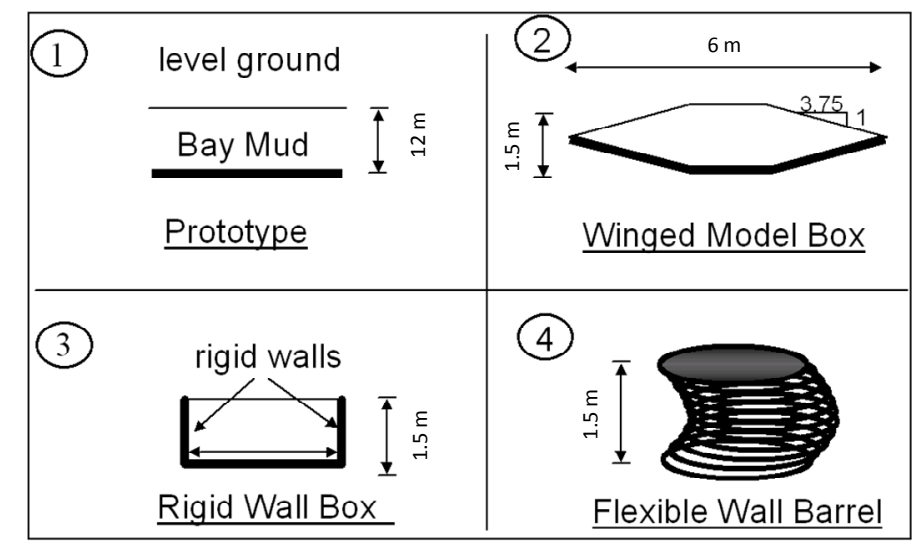

Figure 4-11 Different model soil containers for SSI shake table testing (after Meymand, 1998) 


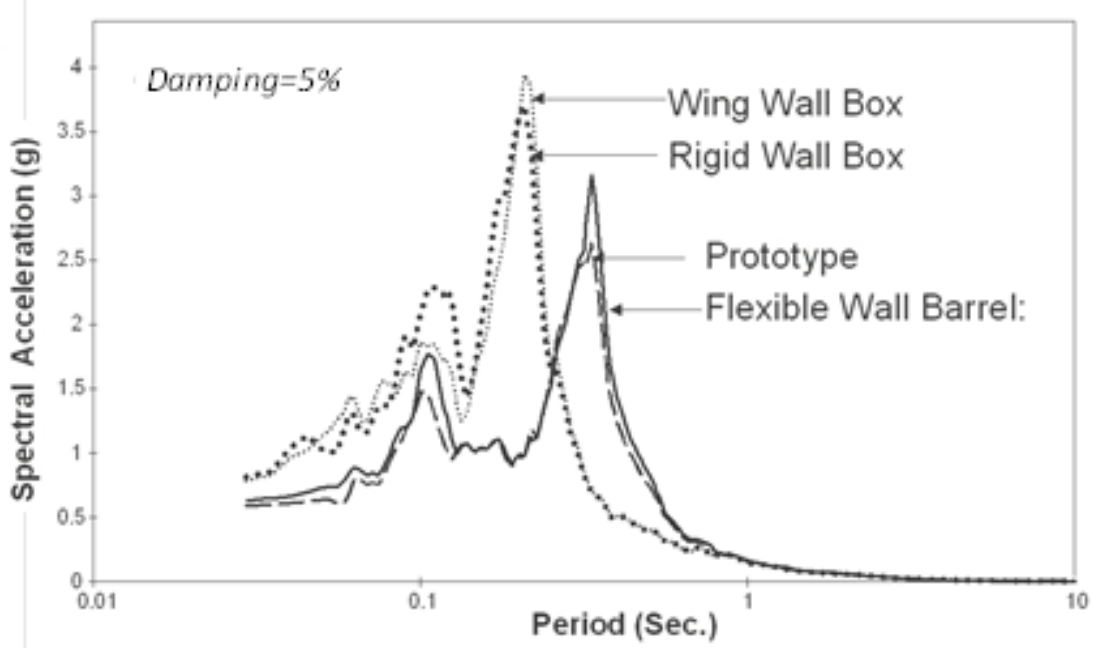

Figure 4-12 Dynamic analysis of different model soil containers showing that the flexible wall barrel provides similar response when compared to prototype field conditions (after Meymand, 1998)

The flexible wall barrel was originally designed and developed by Meymand (1998) at University of California, Berkeley for SSPFI research on Berkeley's large scale shake table. The testing equipment is on loan for further SSI shake table testing at Cal Poly, and several components of the container have been modified and refurbished by Crosariol (2010) in order to fit the smaller Cal Poly shake table. The restructured wall barrel stands 1.5 meters tall and has an inside diameter of 2.3 meters. The fully assembled testing container is presented in Figure 4-13.

The modified testing container is assembled with four shortened steel columns supporting a top ring with universal joints to allow for full horizontal, translational, and rotational freedom of the soil column (Meymand 1998). The soil column is confined by a $6.4 \mathrm{~mm}$ thick open ended neoprene rubber cylinder fastened on the top and the bottom rings with inner compression rings. Additional confinement is provided by a succession of $45 \mathrm{~mm}$ wide woven spectra fiber bands spaced on center $60 \mathrm{~mm}$ around the rubber membrane. These additional bands are designed to carry 
hoop stress induced by static and dynamic loads from the soil column and reduce irregular bulging of the rubber (Crosariol 2010). The test container has full lateral flexibility and radial stiffness provided by the combination of the neoprene membrane and the woven bands (Meymand 1998). For details on the complete refurbishing process of the testing container, please refer to Crosariol's study.

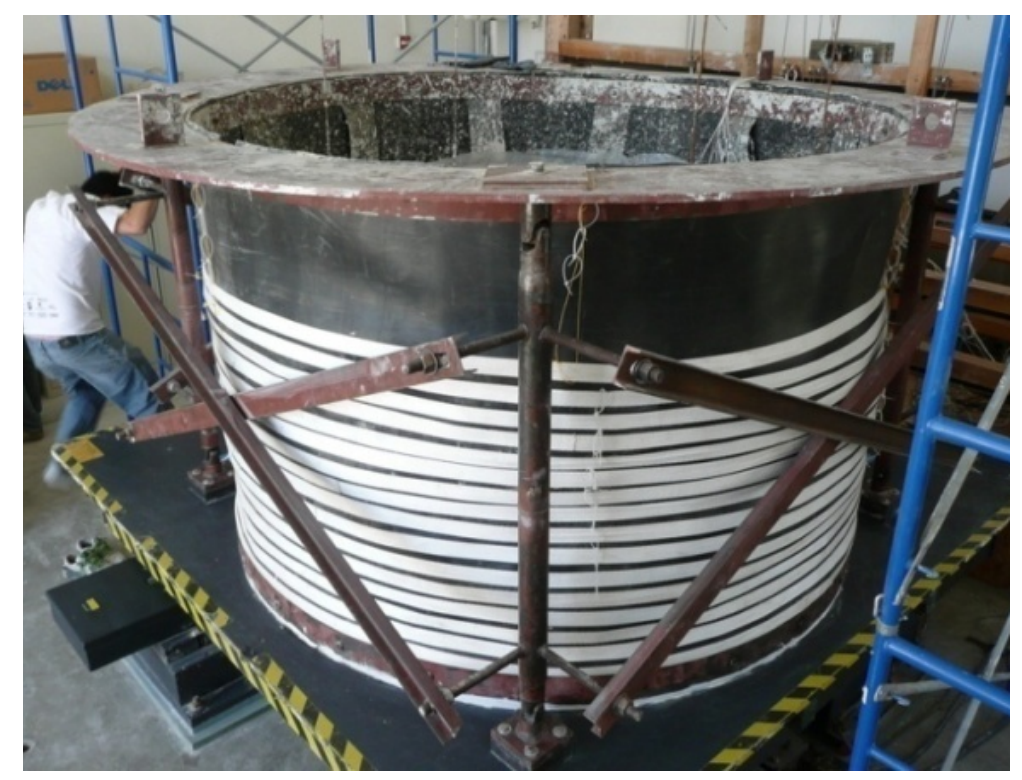

Figure 4-13 The testing platform shown consists of the shake table, the flexible wall barrel, and the associated elements. Located at Cal Poly's Parsons Earthquake Lab

\section{CHAPTER 5 - EXPERIMENTAL TESTING PROGRAM}

\subsection{Introduction}

The testing of this research project was divided into two phases. During Phase I, the model was fixed to a small shake table to capture the natural periods of vibration of the scale model in order to serve as a baseline response. In Phase II on the large shake 
table, the same model was embedded into the soil column in the testing container at various depths, and the free field data were collected simultaneously with the structure response for direct comparison. All these data sets were then used to determine the effects of embedment depth on the seismic response of the scale model.

Much of the information included here was modified after studies from Crosariol (2010), who designed the first shake table testing program at Cal Poly. Information on the testing equipment, instrumentation setup and procedures, and schedule are provided in this chapter.

\subsection{Phase I: Fixed-base Approach}

Phase I of this study was performed on the smaller one-directional shake table in the Advance Soils Lab at Cal Poly shown in Figure 5-1. The purpose was to confirm natural periods of vibration and dynamic responses of the scale model using experimental modal analysis. The scale model was secured onto the shake table to mimic a "fixedbase" condition, where the overall seismic response was only dependent upon the structure itself.

The initial lumped mass height and weight configuration of the SDOF (singledegree-of-freedom) oscillation were determined from analytical equations with an assumption of $5 \%$ resonance damping in the system. Various test runs were conducted to find configurations that best fit the target 2-3 story small building prototype with natural periods of vibration of $0.15 \mathrm{~s}$ and $0.25 \mathrm{~s}$. An accelerometer was attached to the lumped mass to capture acceleration data as seen in Figure 5-2. Accelerometer (Model J353B51 ICP made by PCB Piezotronics) was used to record the mass acceleration data with respect to the driving frequency of the shake table. 
During modal testing, a sine sweep frequency from $0.45 \mathrm{~Hz}$ to $2.0 \mathrm{~Hz}$, with an increment of $0.05 \mathrm{~Hz} / \mathrm{s}$, was input into the shake table and run until the "lollipop", or SDOF oscillator, reached the first mode of natural period. The occurrence of first modal resonance was observed when the "lollipop" went through a cycle of vigorous vibration and reached the first mode shape. In addition, the exact first mode of vibration could be deduced from the mass acceleration in log scale versus driving frequency plot. When the SDOF lumped mass experienced maximum mass acceleration, the corresponding driving frequency represented a close approximation of the first mode of natural period. The experimental modal analysis yielded desired results of optimal lumped mass and height configurations to correspond to the desired prototype at the $10^{\text {th }}$ geometric scale.

The final configuration of the scale model used a $9.07 \mathrm{~kg}$ weight at $52 \mathrm{~cm}$ and 72 $\mathrm{cm}$ height from the center of the weight to the top of the foundation plate to achieve a prototype fundamental period of $0.15 \mathrm{~s}$ and $0.25 \mathrm{~s}$, respectively. The $72 \mathrm{~cm}$ setup was noted as model $(\mathrm{H})$, and the $52 \mathrm{~cm}$ setup was distinguished as model (L) during Phase II testing. The height of the weight plate could be adjusted by loosening and tightening the nuts at the desired locations on the treaded rod. 


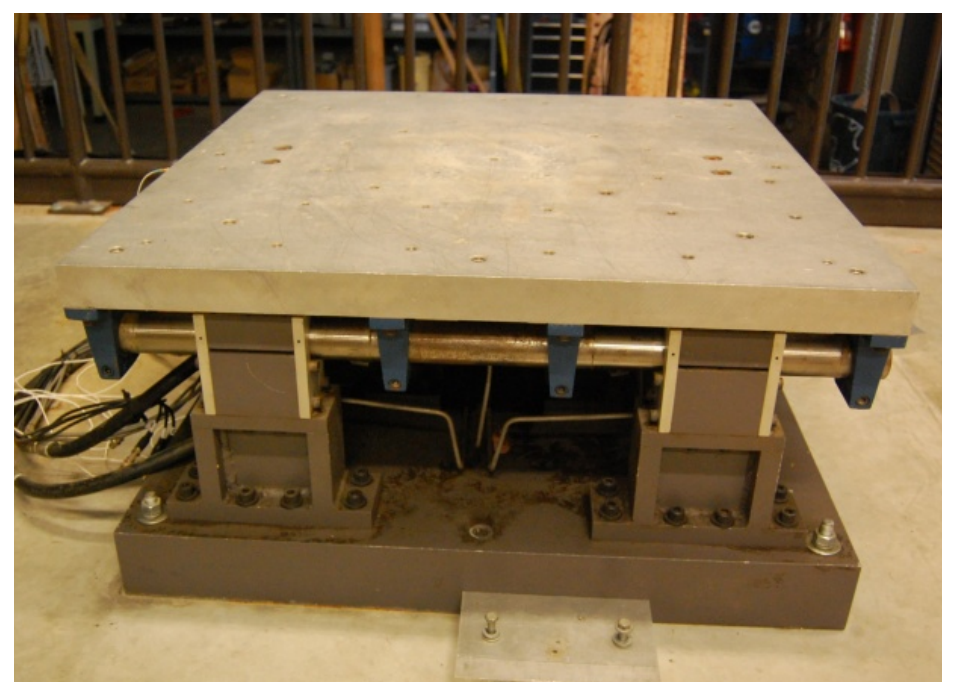

Figure 5-5-1 Small shake table used in the Phase I study. Input motions were controlled by Dactron shaker controller program from a nearby computer

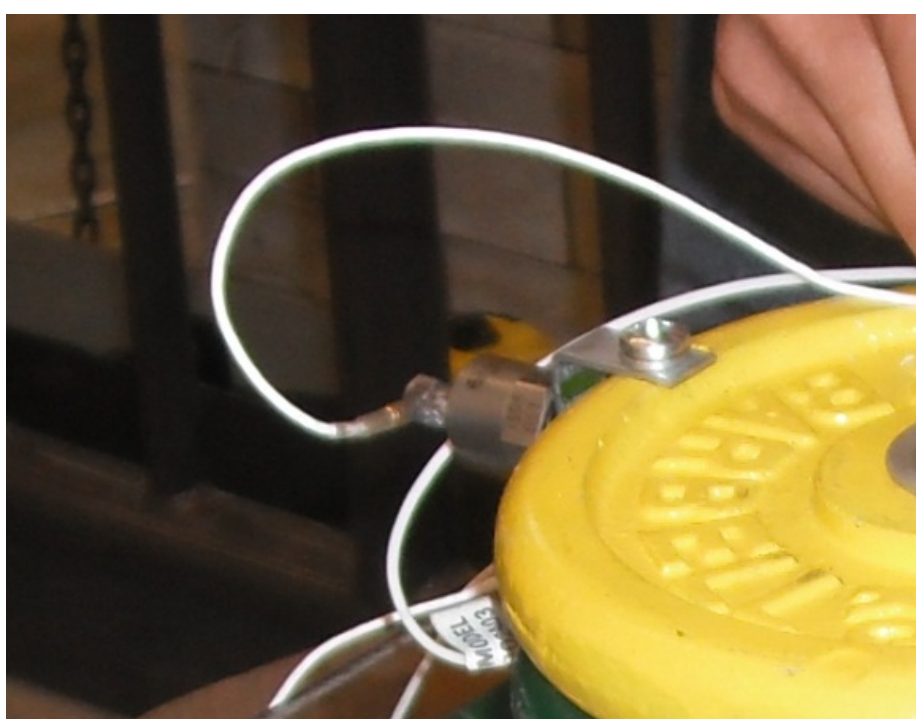

Figure 5-2 An accelerometer was attached to the side of the weight plates to collect acceleration data during the sine sweep test

\subsection{Phase II: Flexible-base Approach}

During Phase II, the anticipated flexible-base condition was simulated by placing and embedding the scale model into the model soil. Inertial interaction effects from SFSI were studied for three different experimental conditions: 1) no foundation embedment, 2) half-embedment with an embedment ratio $>0.5$, and 3) full embedment with an 
embedment ratio $>1.0$. The decision to not include testing of embedment ratio $<0.5$ was based on the Stewart et al. (1998) finding that inertial interaction is negligible for $\mathrm{e} / \mathrm{r}<$ 0.5 .

\subsubsection{Large Shake Table}

The Parsons Earthquake Lab at Cal Poly has a one-dimensional shake table with a $9000 \mathrm{~kg}$ payload capacity. With the maximum payload, the table can accelerate up to $1 \mathrm{~g}$, have a maximum velocity of $97 \mathrm{~cm} / \mathrm{sec}$ and a maximum peak to peak displacement of 25 $\mathrm{cm}$, and can operate in the frequency range of 0.1 to $50 \mathrm{~Hz}$. A full flexible wall barrel and accompanying testing equipment have been estimated to weigh around $3500 \mathrm{~kg}$. Figure 5-3 shows the setup of the shake table facility including the controller area, shake table with the testing container setup, data acquisition module, and other equipment in the lab.

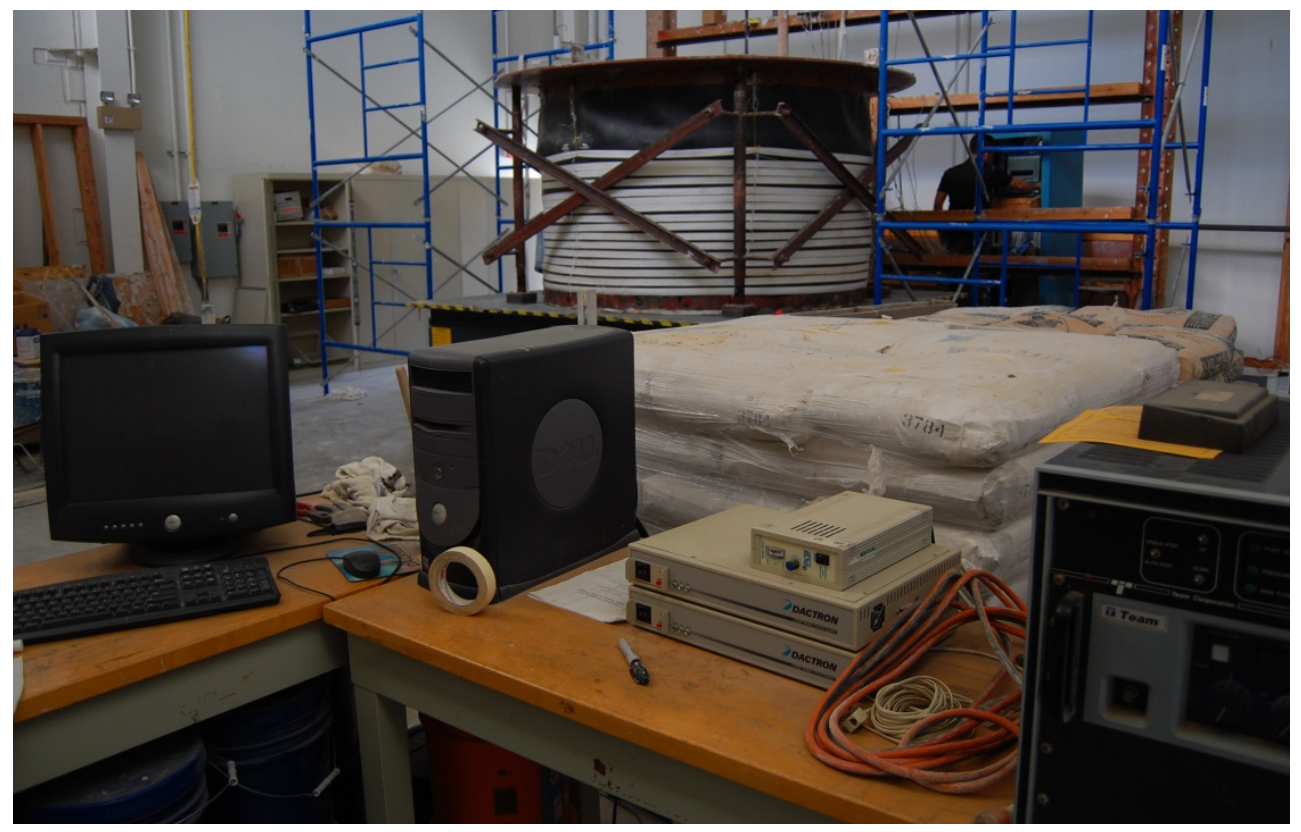

Figure 5-3 Snapshot of Parson's Earthquake and Geotechnical Engineering Laboratory at Cal Poly 
The data acquisition unit and signal calibration module operate on a PC equipped with a National Instruments SCXI 1001 chassis, which has twelve slots for SCXI signal units. Data acquisition software National Instruments NI-DAQ allows for signal calibration and adjusts to the desired data range. The test monitoring software LabView allows for real time data recording and monitoring. Figure 5-4 shows the coaxial cables coming from all the instrumentations used with ID tags connecting to the SCXI 1001 chassis.

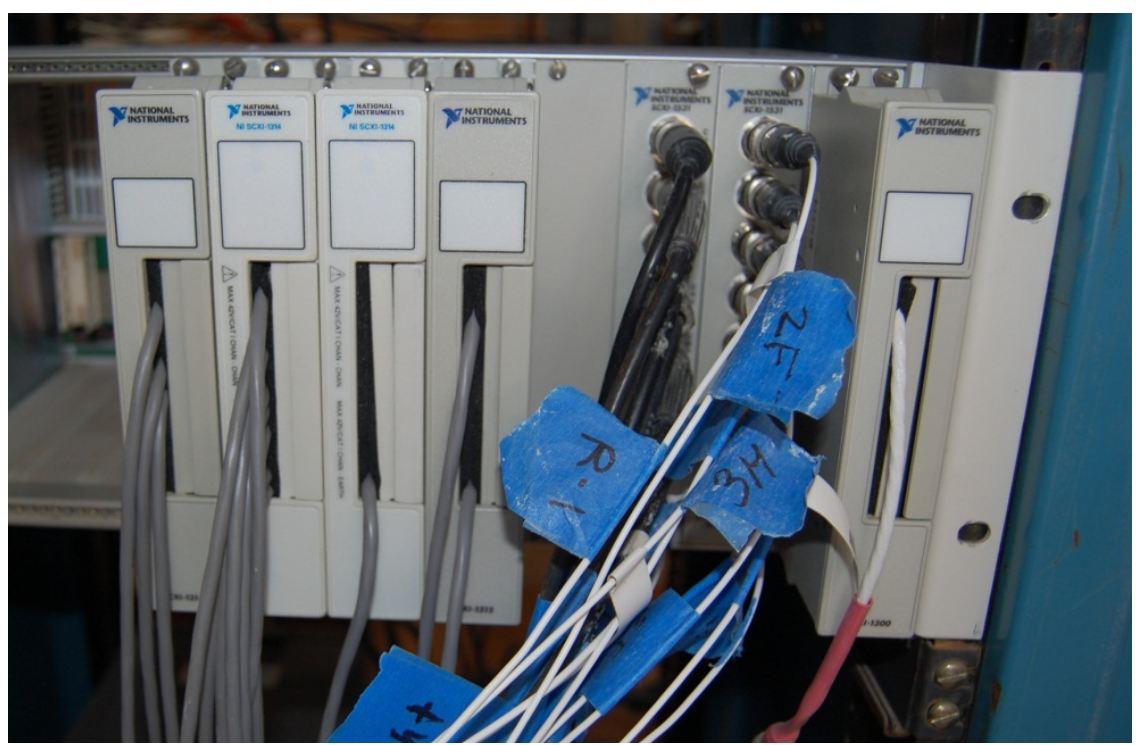

Figure 5-4 A close up view of the SCXI 1001 chassis that receives voltage signals through the instrumentation cables and converts to appropriate measuring values

\subsection{Test Instrumentation}

The majority of the setup in this study followed the works of Crosariol (2010). This section includes information pertinent to this study only. For a more detailed explanation on the shake table configuration, please refer to that study. 


\subsubsection{Accelerometers}

All the accelerometers used in this study were manufactured by PCB Piezotronics. Three different types of accelerometers were selected, and each type was used to measure acceleration for different purposes.

These accelerometers contain sensing elements that respond to applied mass acceleration and produce a proportional electrical output. The electrical voltage is then transmitted through a coaxial cable connecting to the data acquisition unit and converted to acceleration measurements, usually in gravitational unit $(\mathrm{g})$ through a pre-calibrated voltage sensitivity setting. Each accelerometer was calibrated and tested for accuracy prior to Phase II testing.

A single model 353B52 ICP (Integrated Circuit Piezolectric) accelerometer was mounted to the shake table to record table output acceleration data and to provide calibration feedback to the table control during operation. Figure 5-4 shows the accelerometer attached to the back of the shake table.

There were 10 model 393B04 accelerometers used to measure acceleration in the soil column and on the soil surface. These accelerometers were mounted into minifoundations made from acrylic plastic to minimize movement and rotation within the soil and to aid with placement during installation (Crosariol 2010). Due to the highly corrosive nature and saturated condition of the soft clay soil, accelerometers were heavily coated and re-coated with silicon sealant for protection. Figure 5-5 shows a few accelerometers in the acrylic board housing with visible silicon sealant protection around the accelerometer and the wire connection. 


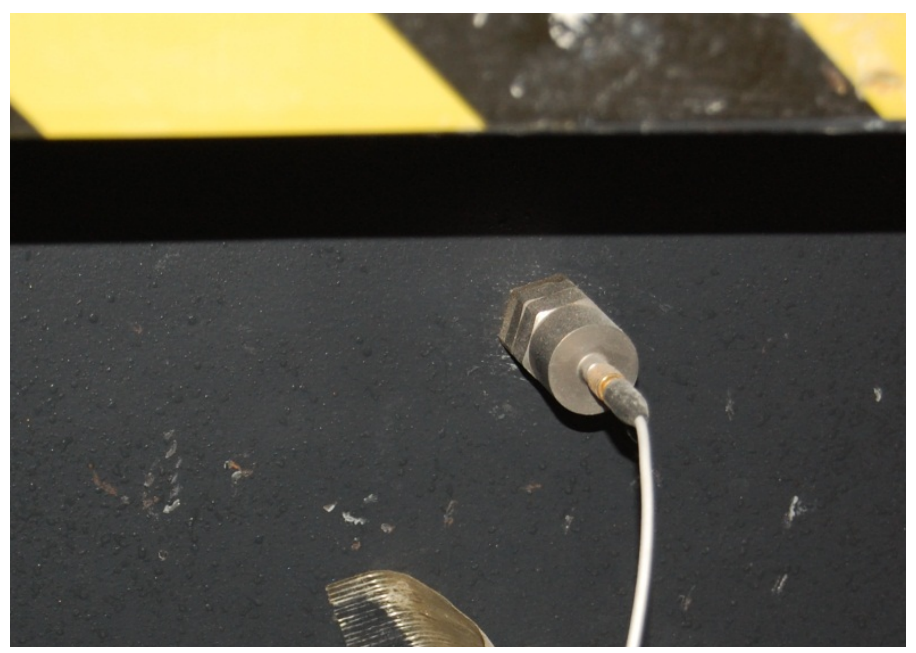

Figure 5-5 Accelerometer 353B52 used to measure the shake table output acceleration

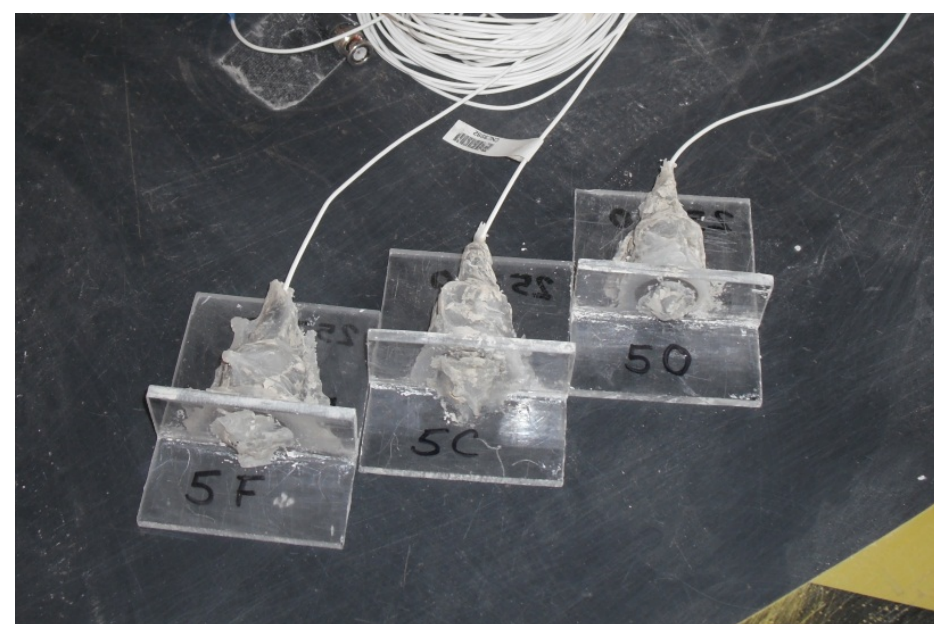

Figure 5-6 Accelerometers 393B04 with the mini-foundation housing and silicon sealant protection

Five model J353B51 accelerometers were used to measure acceleration in the scale model. As mentioned earlier, one accelerometer was attached to the weight plates, which served as the lumped mass of the SDOF oscillator. Two accelerometers were used to measure the horizontal acceleration, and the last two were used to measure foundation rocking behavior during seismic loading. Details of the placement of these accelerometers are described in a later section, and a sample picture of accelerometer J353B51 is shown in Figure 5-7. 


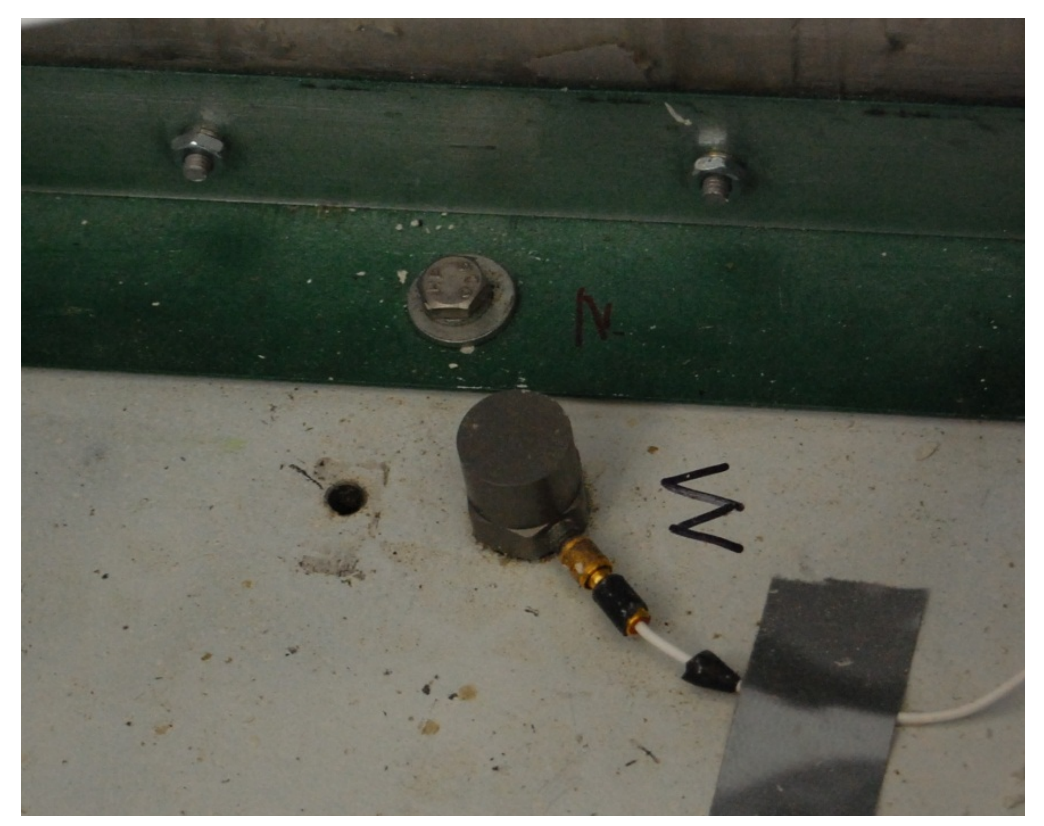

Figure 5-7 Accelerometer J353B51 used as a foundation rocking monitor

\subsubsection{Wire Potentiometers}

Two wire potentiometers (also known as “wire pots,” model PN 62-60-8141), manufactured by SpaceAge Control Inc., were used to measure the absolute horizontal displacements of the soil column and shake table during testing. The pots have internal tensioned coiled wire, which produces voltage changes as the wire is pulled or retracted. The voltage difference is then converted to displacement data through the data acquisition system.

Wire pots were connected at the surface of the shake table and at the level of the soil surface to measure the relative lateral displacement. They were labeled as WPT for the top position measurement of the soil surface, and WPL for the lower position measurement of the shake table surface. These two wire pots were fixed to a 2' by 4' wooden stud with the auto retractable wire end attached at the desired locations with the 
use of finishing line, swivels, zip ties, and duct tape. Figure 5-8 shows the top wire pot setup with the wire connecting to the bucket.

The displacement data collected is not exactly precise due to the elasticity of the testing container, which can deform between tests from the lateral soil movement during shake table testing. The displacement data had to be zeroed to baseline to capture the actual displacement. These wire pots were found to be insufficient for measuring accurate differential soil displacement with depth, but served well to approximate the soil displacement during seismic loading (Crosariol 2010). These displacement measurements are used to compare the maximum relative displacement of the soil surface to the shake table. Results are discussed in Chapter 6.

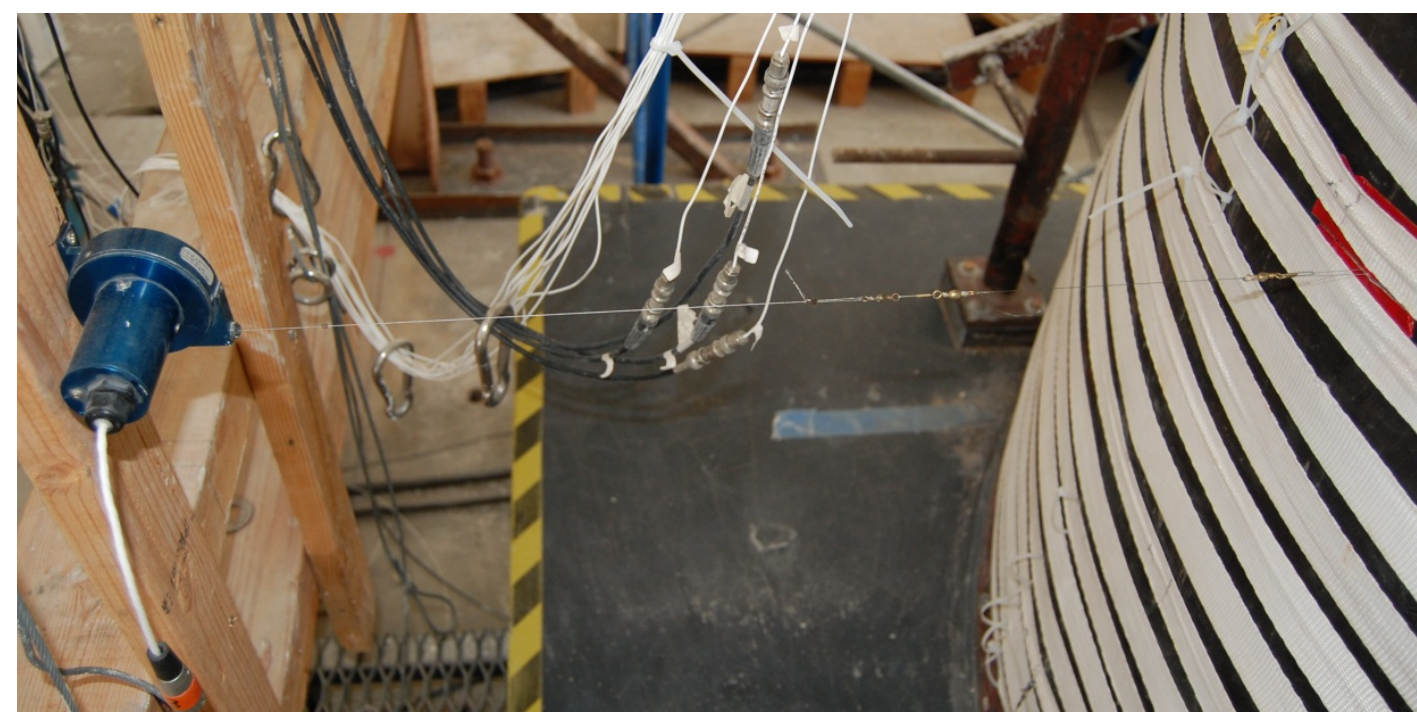

Figure 5-8 The top wire pot is connecting to a hook on the surface of the rubber at the same level as the surface of the soil column to measure displacement during testing

\subsubsection{T-bar Penetrometer}

Stewart and Randolph (1991) developed the T-bar penetrometer device for measuring continuous soil strength with depth. Various validation studies of this empirical estimation method have been conducted, and results were favorable for strength 
measurements in this study (Meymand 1998; Crosariol 2010). A T-bar is an inverted cylindrical T-shaped bar that is pulled or pushed through soil, and the resistance is measured using a load cell. Undrained shear strength can be measured from the load cell, as demonstrated in an earlier study of Randolph and Houlsby (1984). Meymand (1998) further developed the particular T-bar used for the study within by accounting for the bar factor $\left(N_{b}\right)$ and the diameter of the T-bar cylinder (D) using equation 5.1.

$$
S_{u}=\frac{P}{N_{b} D}
$$

Where $S_{u}=$ undrained shear strength of the soil, $P=$ force per unit length acting on the cylinder.

The bar factor was assumed to be 10.5, as suggested by Randolph and Houlsby (1984). The T-bar is made out of a steel rod, with dimension of $95 \mathrm{~mm}$ in length and 19 mm diameter, welded perpendicularly to a 2.1 meter long and $6.33 \mathrm{~mm}$ diameter pulling rod. A load cell model SSC-500-0000, by Tovey Engineering Inc., was used for the T-bar pull out resistance measurement. Figure 5-9 shows the detail of the T-bar configuration.

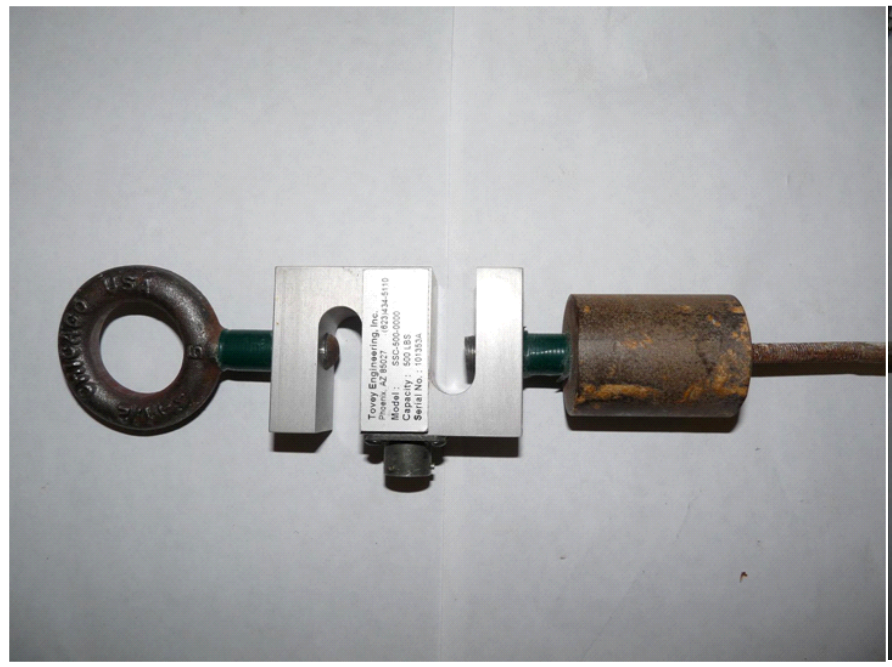

(a)

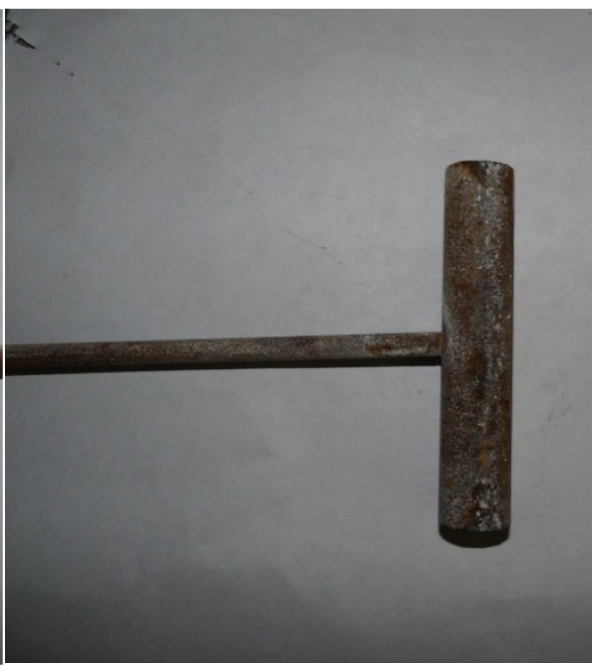

(b) 
Figure 5-9 T-bar testing device with (a) $2.2 \mathrm{kN}$ load cell threaded to T-bar rod and eye bolt for pulling using the crane and (b) T-bar penetrometer steel rod that is embedded in the soil ( From Crosariol 2010)

\subsection{Phase II Setup}

Phase II setup took considerable time due to the physically demanding soil filling process, scale model installation, and instrumentation calibration and placement. Although the procedures were mostly adopted from Crosariol (2010), the process of completing the setup was still labor intensive. Specific details pertinent to Phase II setup for this study are presented below.

\subsubsection{Soil Mixing and Placement}

The target soil column was $100 \mathrm{~cm}$ tall. As mentioned in Chapter 4, the model soil used here was originally mixed by Crosariol (2010). The soil was reconstituted and carefully hand packed into the testing container in a careful manner to minimize voids and achieve desired consistency. A series of samples were collected at each lift of installment, usually at $10 \mathrm{~cm}$ increments, to measure the water content of the model soil. Based on the samples collected, the average water content for the soil column was $115 \%$. Figure 5-10 shows the soil water content throughout the soil column for this study.

There was insufficient leftover reconstituted model soil to fill up the entire testing container; thus, a new batch of model soil had to be made. The decrease in soil volume was primarily caused by clay shrinkage due to drying and loss of soil while transferring during a previous shake table research. The water content of the new batch had a higher water content than the reconstituted soil because a minimum of $120 \%$ water content was necessary for efficient pump operation. 


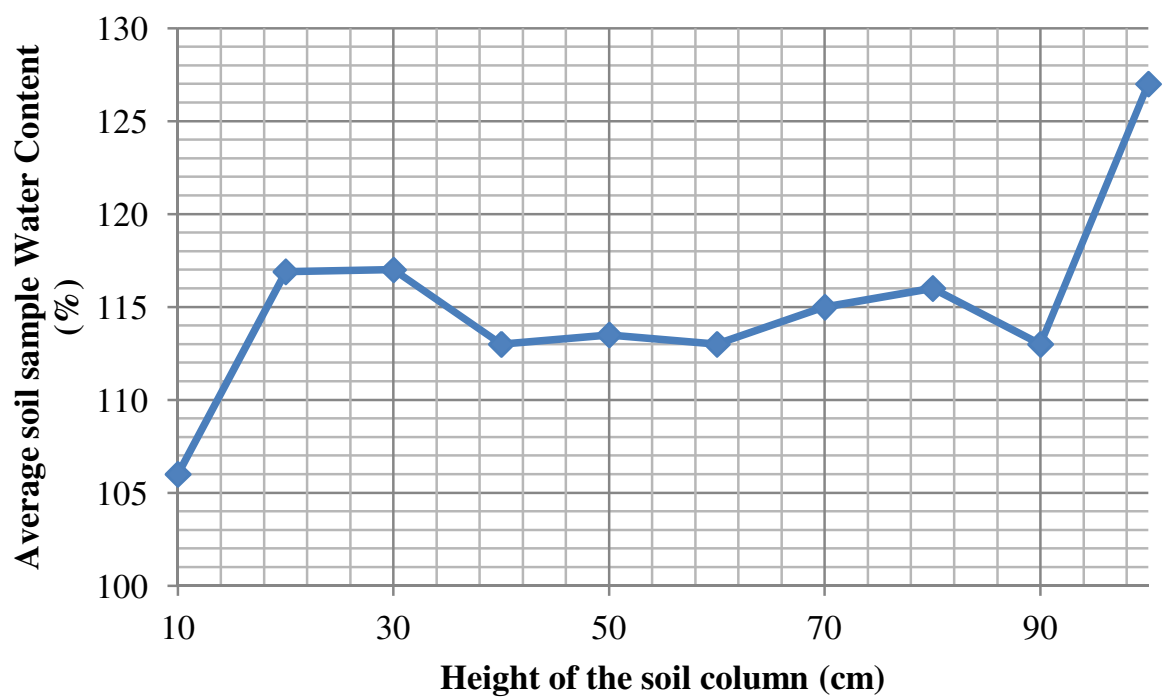

Figure 5-10 Soil water content in the testing container

\subsubsection{Placement and Embedment of Scale Model}

The scale model was put in place at the predetermined location using an overhead crane. A threaded eye-bolt was secured into the foundation steel plate and connected to the crane using a Kevlar strap. Once the scale model was at the desired orientation, with guide rails running parallel to the shaking direction, the eye-bolt was unscrewed and replaced with the threaded steel rod for the "lollipop". Slight settlement occurred due to the weight of the foundation but no further consolidation issues were considered for the undrained soil condition. The heavy weight of the foundation, in addition to the high clay soil cohesion, eliminated the potential for sliding. Figure 5-11 shows the scale model in place on the soil surface without embedment.

The scale model was embedded into the soil by excavating a cavity the exact size of the foundation at the desired embedment depth to minimize soil disturbance. This mimicked the partially cut-and-cover construction method and maintained soil uniformity. The first embedment of $15 \mathrm{~cm}$ was made by digging into the clay with a 
metal trowel. The excavation process was constantly monitored for accurate dimension, and the surface was smoothed using a straight edge. Several vertical grooves were made at corresponding locations of screws on the outer wall of the foundation to provide a good fit. Once the cavity was excavated, the scale model was lowered in place using the crane as seen in Figure 5-12.

The model was guided with care during the lowering process to avoid disrupting surrounding soil. Two levels located on the surface of the steel foundation in both directions provide visual confirmation of the foundation level. For the full embedment of $30 \mathrm{~cm}$, the model was pulled out of the cavity using the overhead crane, and then lowered again into a deeper excavated cavity using the same process. The newly embedded foundation was left for two weeks to prior to shake table testing. A tarp, which has an opening for the dimension of the scale model, was placed on top to minimize evaporation at the soil surface during the two weeks. The tarp was removed during testing.

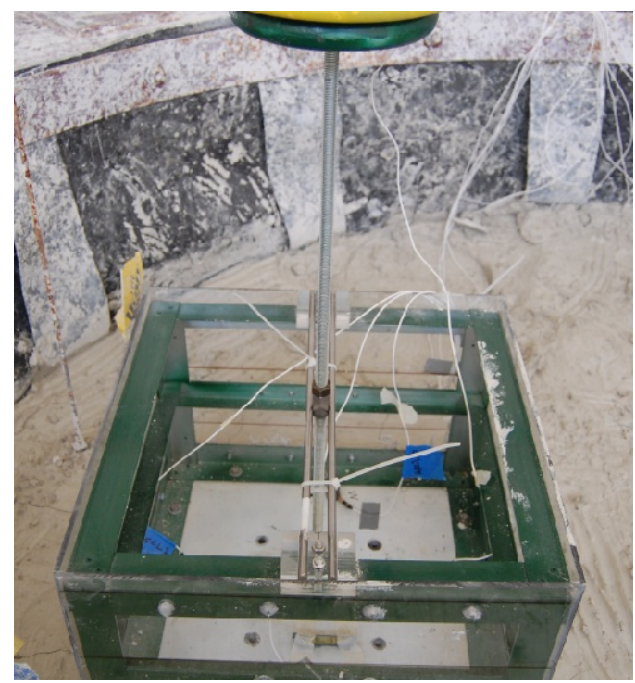

Figure 5-11 Scale model with the foundation resting on the model soil surface in the testing container 


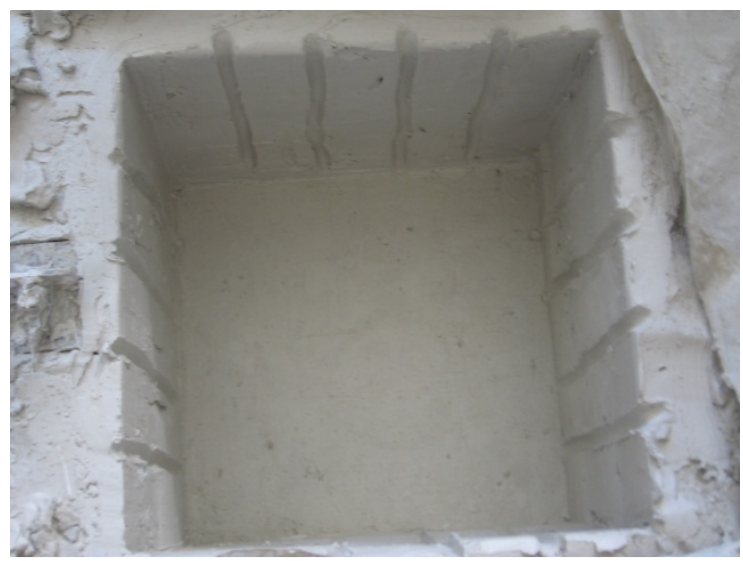

(a)

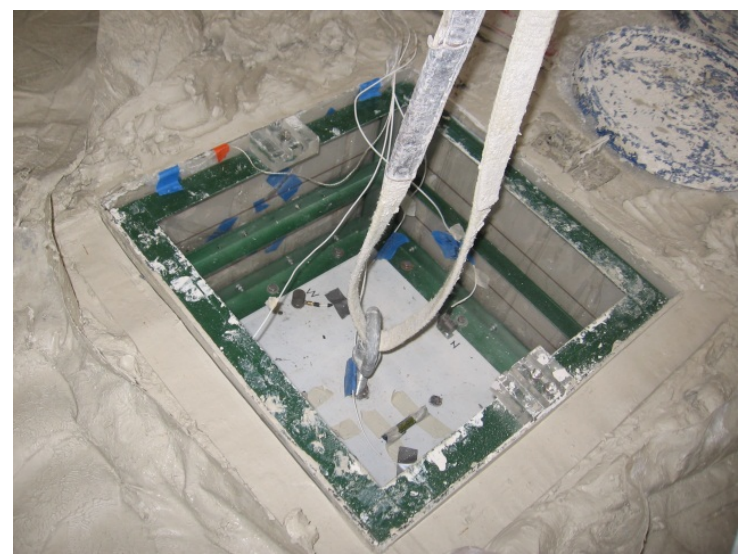

(b)

Figure 5-12 Scale model embedment process with (a) excavation of a cavity in the soil column and (b) lowering the model in place using overhead crane

\subsection{Instrumentation}

\subsubsection{Model instrumentation and Configuration}

Accelerometers were used to record acceleration data of the scale model during testing. A total of five accelerometers were used within the scale model structure as seen in Figure 5-13.

One accelerometer (WT) was mounted on the weight plate in the same manner as Phase I testing. In Phase II testing, the dynamic behavior of the SDOF lumped mass during seismic excitation was recorded.

Two accelerometers (HN, HS) were mounted horizontally on L-brackets to measure foundation acceleration. They were located on the same alignment as the freefield accelerometers and on the north and south sides of the steel plate to provide dual recordings and to also measure twisting in the foundation. Figure 5-14(a) shows the north 
side accelerometer mounted horizontally. Results of the foundation level accelerometers are compared to the free-field surface level accelerometers in Chapter 6.

Two more accelerometers (VW, VE) were used to characterize rocking behavior in the foundation during shaking. They were placed in vertical orientation on the east and west side of the foundation as seen in Figure 5-14(b). Foundation rocking can be a factor in overall dynamic response of the foundation. A brief observation from these recordings and acceleration data analysis is presented in Chapter 6 .

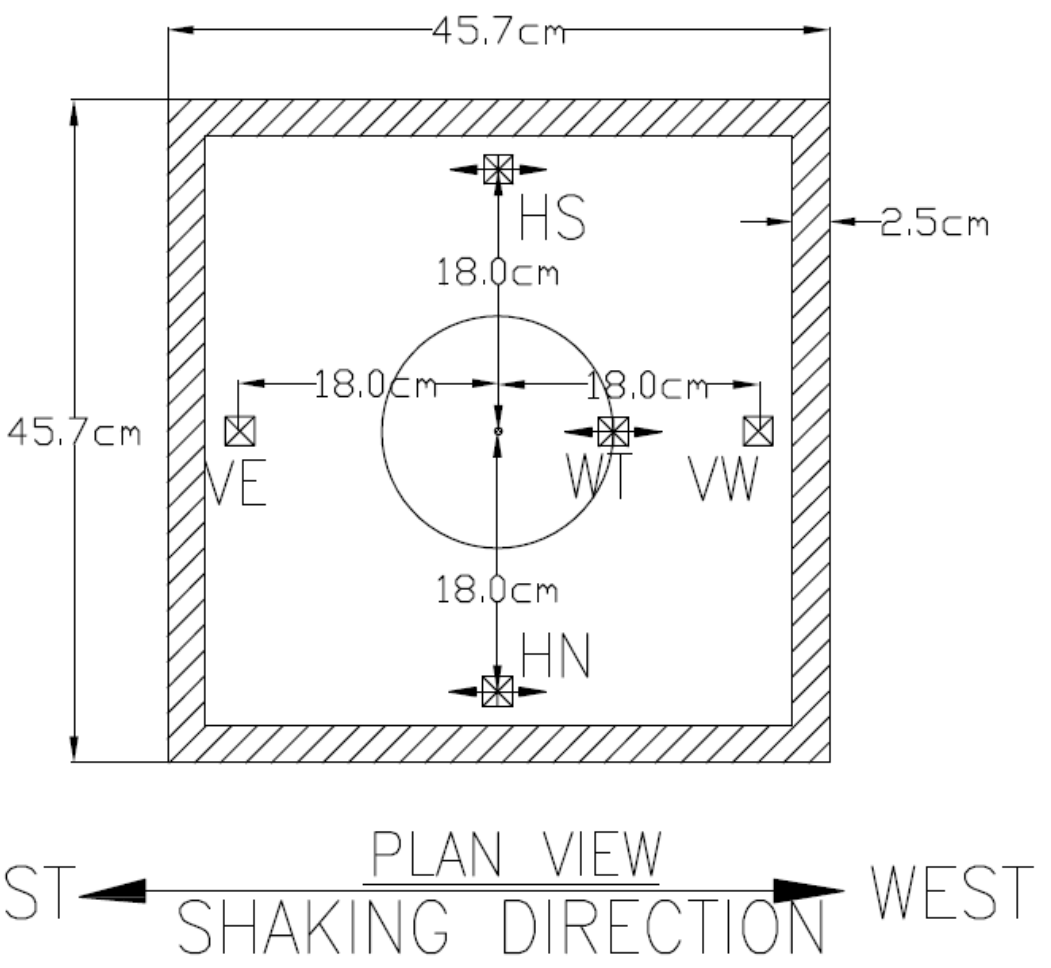

Figure 5-13 Plan view of the scale model with accelerometers placement and dimension

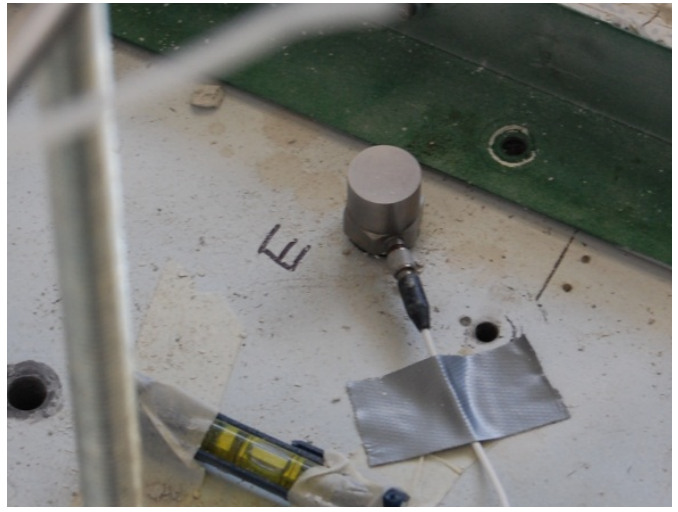




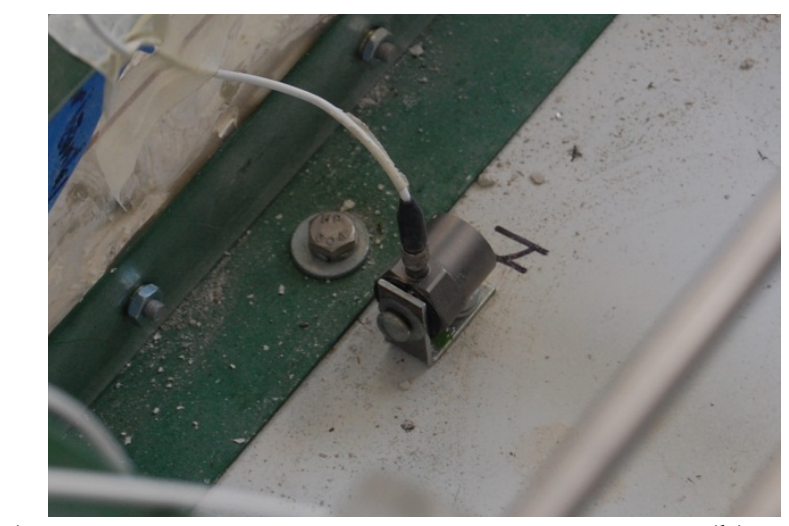

(a)

(b)

Figure 5-14 Accelerometers mounted on the foundation to (a) measure acceleration in the shaking direction and twisting (b) measure vertical acceleration for rocking; the level was used to check the foundation overall level during embedment

\subsubsection{Testing Container instrumentation and Configuration}

The soil column was divided down the middle into a free-field array side and a model array side. The purpose of having two sides was to measure both the free-field array and model array simultaneously during shake table test to provide realistic comparison. A total of 10 accelerometers were placed in the soil column or on the surface. Accelerometers were installed using the method developed by Crosariol (2010) as the testing container was being filled with model soil. Figure 5-15 shows a top view of the testing container with scale model and surface accelerometers located on the soil surface. Details of instrumentation placement locations can be found in Figure 5-16. Table 5-1 provides the specific instrumentation denotation and description. 


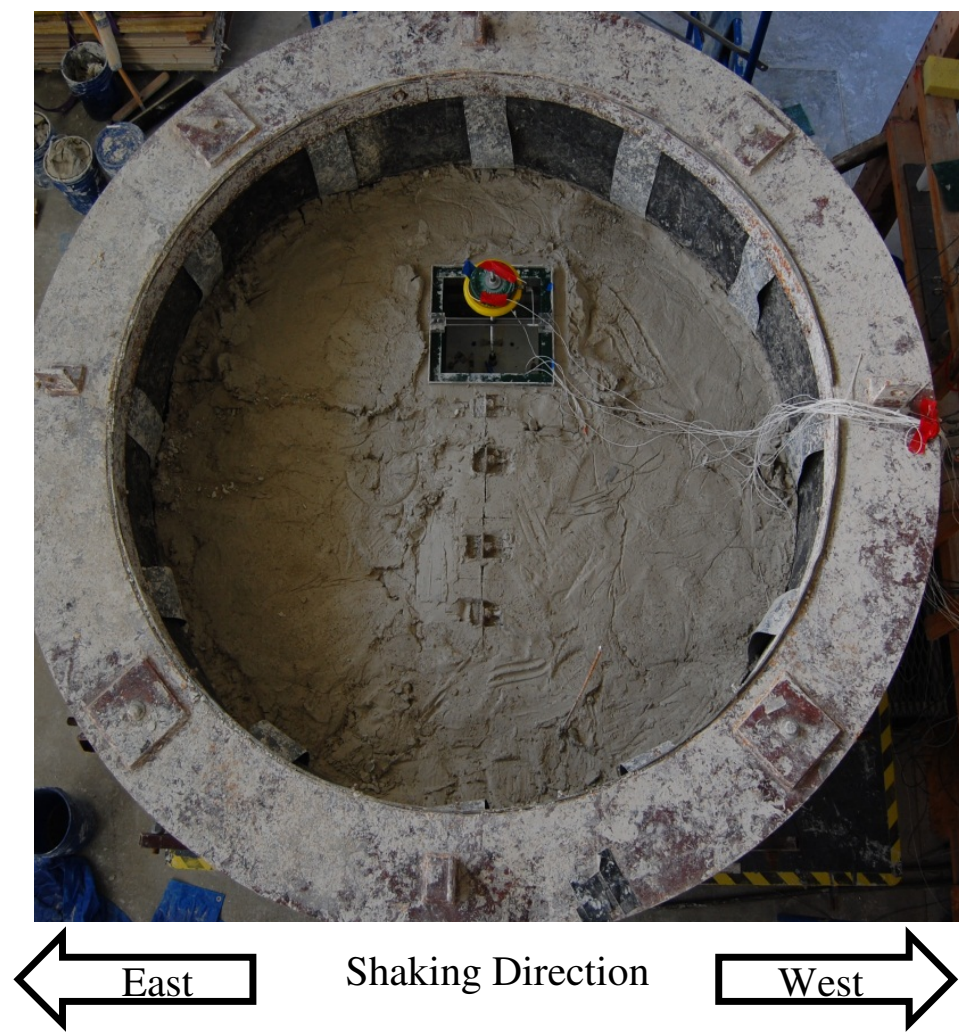

Figure 5-15 Plan view of the testing container with the scale model embedded and surface accelerometers in place 

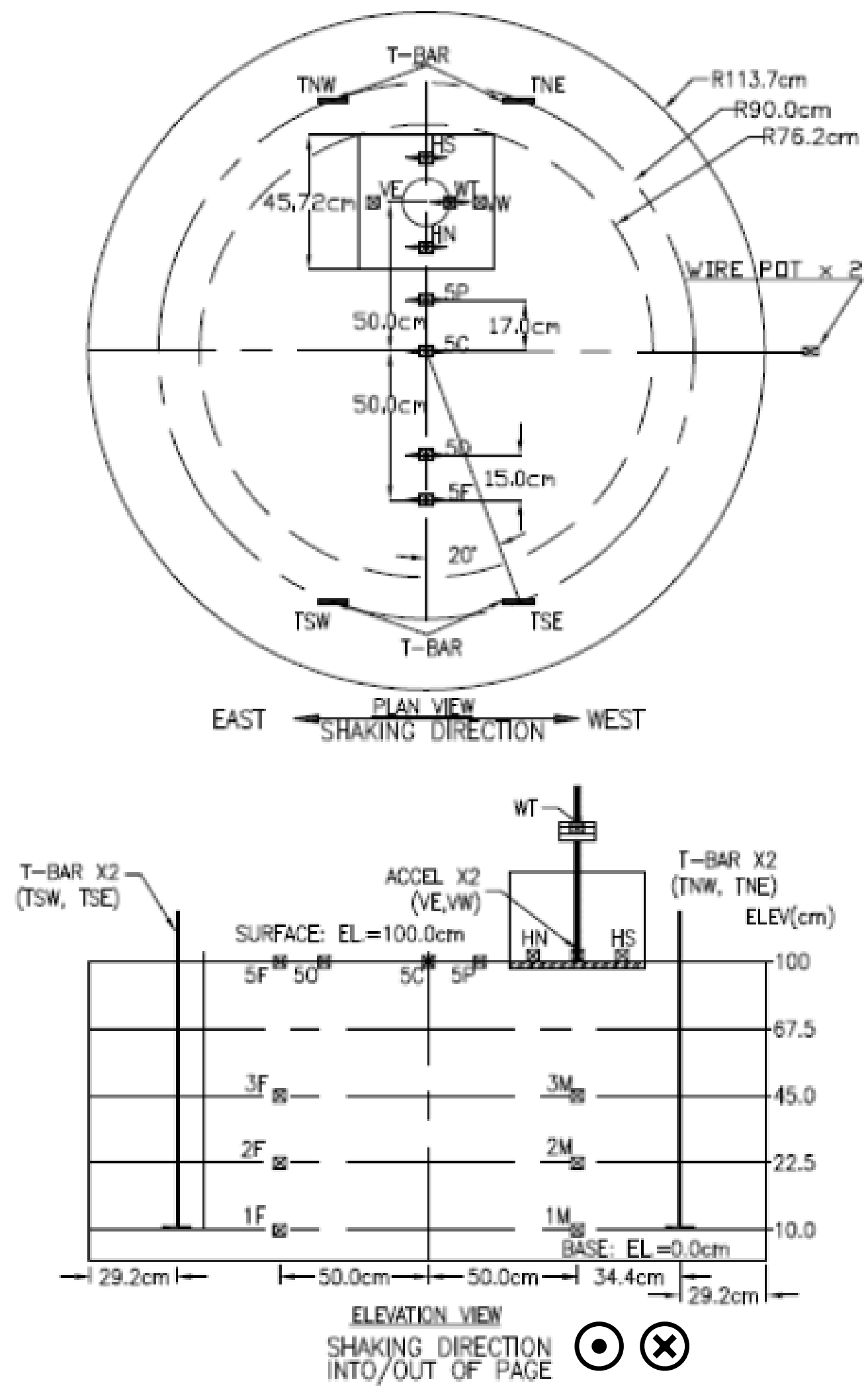

Figure 5-16 Plan and elevation view of the accelerometer placement in the testing container 
Table 5-1 Phase II instrumentation listing and nomenclature (after Crosariol 2010)

\begin{tabular}{|c|c|c|}
\hline Phase II & Instrumentation & Denoted \\
\hline \multirow{4}{*}{$\begin{array}{l}\text { Model Array } \\
\& \text { Foundation }\end{array}$} & $1 \mathrm{ACC}$ on the lumped mass & WT \\
\hline & 2 ACC's on the foundation in horizontal position & HN,HS \\
\hline & 2 ACC's on the foundation in vertical position & VW,VE \\
\hline & 3 ACC's in the model array: levels $1,3,5$ & $1 \mathrm{M}, 2 \mathrm{M}, 3 \mathrm{M}$ \\
\hline \multirow{4}{*}{$\begin{array}{l}\text { Free-field } \\
\text { Array }\end{array}$} & $4 \mathrm{ACC}$ 's in the free-field array: levels $1,2,3,4,5$ & $1 \mathrm{~F}, 2 \mathrm{~F}, 3 \mathrm{~F}, 5 \mathrm{~F}$ \\
\hline & 1 ACC in center position: level 5 & $5 \mathrm{C}$ \\
\hline & 1 ACC in offset position: level 5 & 50 \\
\hline & $1 \mathrm{ACC}$ in proximate position: level 5 & $5 \mathrm{P}$ \\
\hline \multirow[t]{2}{*}{ Soil Column } & 4 T-bars in NE, SE, SW: NW section & $\begin{array}{l}\text { TNE, TSE, } \\
\text { TSW, TNW }\end{array}$ \\
\hline & 2 wire pots (WP): low, high & WPL, WPH \\
\hline
\end{tabular}

\subsection{Test Parameters}

\subsubsection{T-Bar Tests}

Four T-bars were placed vertically in the container before model soil filling at four different corners (northeast, northwest, southeast, southwest). The pull-out test schedule is listed in Table 5-2. Timber cross beams with drilled holes were clamped to the top ring to guide the T-bars in place, and various clamps were used to hold and secure them during soil filling as seen in Figure 5-17. The T-bar pull-out tests were conducted at a one-month interval to measure soil strength gain over time and soil spatial variation.

The T-bars were pulled out of the soil using an overhead crane at a constant rate of $1.29 \mathrm{~cm} / \mathrm{s}$. A load cell hooked onto the crane was attached to the threaded end of the Tbar, and the testing data was recorded at a sample rate of $25 \mathrm{~Hz}$ for the full depth of embedment using the LabView program on the data acquisition module. Results of the Tbar tests are discussed in the Chapter 6. 
Table 5-2 T-bar pull out test for Phase II testing

\begin{tabular}{|c|c|c|c|}
\hline$\#$ & Date & Test_ID & Test Description \\
\hline \hline 1 & $9 / 1 / 2010$ & 0_Tbar-SE & T-Bar pull out test at the Southeast corner \\
\hline 2 & $10 / 1 / 2010$ & 1_Tbar-SW & T-Bar pull out test at the Southwest corner \\
\hline 3 & $11 / 4 / 2010$ & 2_Tbar-NW & T-bar pull out test at the Northwest corner \\
\hline 4 & $12 / 2 / 2010$ & 3_Tbar-NE & T-bar pull out test at the Northeast corner \\
\hline
\end{tabular}

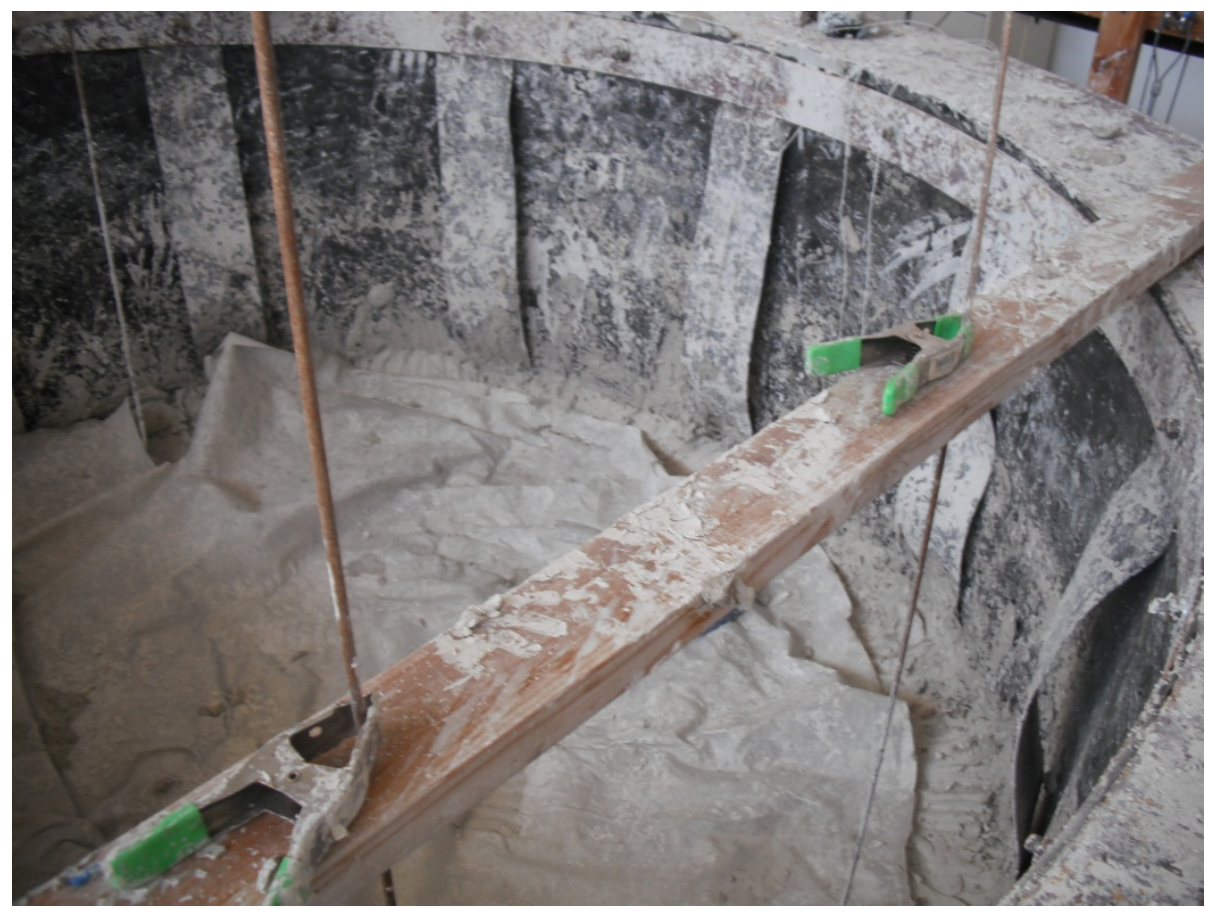

Figure 5-17 Two T-bars being held in place using a wooden beam and two clamps. Once the container is filled, the beam and clamps were removed

\subsubsection{Hammer Blow Tests}

A series of hammer blow tests were performed to estimate the in-situ shear wave velocity in the model soil column. Testing procedures were adopted after Meymand (1998) and Crosariol (2010). A top-down hammer blow approach measured the shear wave velocity of the lower soil column. The bottom-up method resulted in unwanted 
shear wave interference, which was difficult to analyze and therefore omitted. (Crosariol 2010).

A shear wave was induced by striking a framing hammer on a steel bar coupled to the soil surface. Shear wave velocity can be deduced by determining the distances between the accelerometers and the time interval between the wave arrivals at the accelerometers. Waveform interpretation is difficult since waves bounce and interfere with each other. A high sampling rate of $5000 \mathrm{~Hz}$ was necessary to capture high frequency wave forms from the hammer blow. Results of hammer blow testing and shear wave velocity interpretation are discussed in Chapter 6.

\subsubsection{Shake Table Testing Schedule}

A total of 104 shake table tests were conducted for Phase II testing. Excluding calibration and trial test runs, the results from 84 pertinent tests for the scope of this study were examined further. Table 5-3 describes details of Phase II testing schedule. The list includes testing dates, test identification numbers, test descriptions of the motion and intensity used, model setup used, and model embedment condition.

There were four stages of testing conducted. The first was the calibration and trial stage, and the number " 0 " was used to denote it in test ID's. These tests were important to ensure that the shake table was calibrated to run input motions with the testing container setup. Calibration modules from previous study for motions JOS000, JOS090, ELE180, ELE270, TCUW, and TCUN were used, and additional calibration runs were performed for the new motions used in this testing program. Details of all 14 input motions are described in Chapter 4. 
The main testing was performed after the calibration stage. Each series of shake table tests were conducted at a time interval of approximately four weeks to allow the soil to rest. T-bar tests were conducted prior to each stage to measure the model soil's consistency and strength.

The second testing stage was denoted by the number " 1 " in test ID's, and the scale model was placed on the surface of the soil column. The third stage was denoted by the number " 2 " in test ID's, and the model was embedded $15 \mathrm{~cm}$ to achieve an embedment ratio (e/r) of 0.58 . Similarly, the fourth stage was denoted by the number " 3 " in test ID's, and the model was fully embedded to a depth of $30 \mathrm{~cm}$ to reach e/r of 1.16 . In all three testing stages $(1,2$, and 3), 14 motions were used as table input for both model $(\mathrm{H})$ and model (L) setups; therefore, the total tests for each stage was 28 . The results of pertinent tests are analyzed and compared to investigate the influence of embedment on inertial interaction in the following chapter.

Table 5-3 Phase II testing schedule including all four stages

\begin{tabular}{|c|l|l|l|}
\hline$\#$ & \multicolumn{1}{|c|}{ Date } & \multicolumn{1}{c|}{ Test_ID } & \multicolumn{1}{c|}{ Test Description } \\
\hline \hline 1 & $9 / 9 / 2010$ & 0_JOS000 & Calibration for JOS000 At 60\% intensity \\
\hline 2 & $9 / 9 / 2010$ & 0_JOS090 & Calibration for JOS090 At 60\% intensity \\
\hline 3 & $9 / 9 / 2010$ & 0_ELE180 & Calibration for ELE180 At 60\% intensity \\
\hline 4 & $9 / 9 / 2010$ & 0_ELE270 & Calibration for ELE270 At 60\% intensity \\
\hline 5 & $9 / 9 / 2010$ & 0_TCU_W & Calibration for TCUW At 60\% intensity \\
\hline 6 & $9 / 9 / 2010$ & 0_TCU_N & Calibration for TCUN At 60\% intensity \\
\hline 7 & $9 / 14 / 2010$ & 0_JOS000_H & Test with JOS000 at 60\% intensity \\
\hline 8 & $9 / 14 / 2010$ & 0_JOS090_H & Test with JOS090 at 60\% intensity \\
\hline 9 & $9 / 14 / 2010$ & 0_ELE180_H & Test with ELE180 at 60\% intensity \\
\hline 10 & $9 / 14 / 2010$ & 0_ELE270_H & Test with ELE270 at 60\% intensity \\
\hline 11 & $9 / 14 / 2010$ & 0_TCU_W_H & Test with TCU_W at 60\% intensity \\
\hline 12 & $9 / 14 / 2010$ & 0_TCU_N_H & Test with TCU_N at 60\% intensity \\
\hline 13 & $9 / 30 / 2010$ & 1_JOS000_L & Model (L) at no embedment with JOS000 At 100\% intensity \\
\hline 14 & $9 / 30 / 2010$ & 1_JOS090_L & Model (L) at no embedment with JOS090 At 100\% intensity \\
\hline 15 & $9 / 30 / 2010$ & 1_ELE180_L & Model (L) at no embedment with ELE180 At 100\% intensity \\
\hline
\end{tabular}




\begin{tabular}{|c|c|c|c|}
\hline \# & Date & Test_ID & Test Description \\
\hline 16 & $9 / 30 / 2010$ & 1_ELE270_L & Model (L) at no embedment with ELE270 At $100 \%$ intensity \\
\hline 17 & $9 / 30 / 2010$ & 1_TCU_W_L & Model (L) at no embedment with TCUW At $100 \%$ intensity \\
\hline 18 & $9 / 30 / 2010$ & 1_TCU_N_L & Model (L) at no embedment with TCUN At $100 \%$ intensity \\
\hline 19 & $9 / 30 / 2010$ & 1_JOSO00_H & Model $(\mathrm{H})$ at no embedment with JOS000 At $100 \%$ intensity \\
\hline 20 & $9 / 30 / 2010$ & 1_JOS090_H & Model $(\mathrm{H})$ at no embedment with JOS090 At $100 \%$ intensity \\
\hline 21 & $9 / 30 / 2010$ & 1_ELE180_H & Model $(\mathrm{H})$ at no embedment with ELE180 At $100 \%$ intensity \\
\hline 22 & $9 / 30 / 2010$ & 1_ELE270_H & Model $(\mathrm{H})$ at no embedment with ELE270 At $100 \%$ intensity \\
\hline 23 & $9 / 30 / 2010$ & 1_TCU_W_H & Model $(\mathrm{H})$ at no embedment with TCUW At $100 \%$ intensity \\
\hline 24 & $9 / 30 / 2010$ & 1_TCU_N_H & Model $(\mathrm{H})$ at no embedment with TCUN At $100 \%$ intensity \\
\hline 25 & $10 / 1 / 2010$ & 0_CPM000 & Calibration for CPM000 At $60 \%$ intensity \\
\hline 26 & $10 / 1 / 2010$ & 0_CPM090 & Calibration for CPM090 At 60\% intensity \\
\hline 27 & $10 / 1 / 2010$ & 0_LGP000 & Calibration for LGP000 At $60 \%$ intensity \\
\hline 28 & $10 / 1 / 2010$ & 0_LGP090 & Calibration for LGP090 At 60\% intensity \\
\hline 29 & $10 / 1 / 2010$ & 0_L09000 & Calibration for L09000 At $60 \%$ intensity \\
\hline 30 & $10 / 1 / 2010$ & 0_L09090 & Calibration for L09090 At 60\% intensity \\
\hline 31 & $10 / 1 / 2010$ & 0_IPV045 & Calibration for IPV0 45 At $60 \%$ intensity \\
\hline 32 & $10 / 1 / 2010$ & 0_IPV134 & Calibration for IPV134 At $60 \%$ intensity \\
\hline 33 & $10 / 7 / 2010$ & 1_CPM000_L & Model (L) at no embedment with CPM000 At $100 \%$ intensity \\
\hline 34 & $10 / 7 / 2010$ & 1_CPM090_L & Model (L) at no embedment with CPM090 At 100\% intensity \\
\hline 35 & $10 / 7 / 2010$ & 1_LGP000_L & Model (L) at no embedment with LGP000 At $100 \%$ intensity \\
\hline 36 & $10 / 7 / 2010$ & 1_LGP090_L & Model (L) at no embedment with LGP090 At $100 \%$ intensity \\
\hline 37 & $10 / 7 / 2010$ & 1_L09000_L & Model (L) at no embedment with L09000 At $100 \%$ intensity \\
\hline 38 & $10 / 7 / 2010$ & 1_L09090_L & Model (L) at no embedment with L09090 At $100 \%$ intensity \\
\hline 39 & $10 / 7 / 2010$ & 1_IPV045_L & Model (L) at no embedment with IPV045 At $100 \%$ intensity \\
\hline 40 & $10 / 7 / 2010$ & 1_IPV134_L & Model (L) at no embedment with IPV134 At 100\% intensity \\
\hline 41 & $10 / 7 / 2010$ & 1_CPM000_H & Model $(\mathrm{H})$ at no embedment with CPM000 At $100 \%$ intensity \\
\hline 42 & $10 / 7 / 2010$ & 1_CPM090_H & Model $(\mathrm{H})$ at no embedment with CPM090 At 100\% intensity \\
\hline 43 & $10 / 7 / 2010$ & 1_LGP000_H & Model $(\mathrm{H})$ at no embedment with LGP000 At $100 \%$ intensity \\
\hline 44 & $10 / 7 / 2010$ & 1_LGP090_H & Model $(\mathrm{H})$ at no embedment with LGP090 At 100\% intensity \\
\hline 45 & $10 / 7 / 2010$ & 1_L09000_H & Model $(\mathrm{H})$ at no embedment with L09000 At $100 \%$ intensity \\
\hline 46 & $10 / 7 / 2010$ & 1_L09090_H & Model $(\mathrm{H})$ at no embedment with L09090 At $100 \%$ intensity \\
\hline 47 & $10 / 7 / 2010$ & 1_IPV045_H & Model $(\mathrm{H})$ at no embedment with IPV045 At $100 \%$ intensity \\
\hline 48 & $10 / 7 / 2010$ & 1_IPV134_H & Model $(\mathrm{H})$ at no embedment with IPV134 At $100 \%$ intensity \\
\hline 49 & $11 / 4 / 2010$ & 2_JOSO00_H & Model $(\mathrm{H})$ at $15 \mathrm{~cm}$ embedment with JOS000 At $100 \%$ intensity \\
\hline 50 & $11 / 4 / 2010$ & 2_JOS090_H & Model (H) at $15 \mathrm{~cm}$ embedment with JOS090 At $100 \%$ intensity \\
\hline 51 & $11 / 4 / 2010$ & 2_ELE180_H & Model (H) at $15 \mathrm{~cm}$ embedment with ELE180 At $100 \%$ intensity \\
\hline 52 & $11 / 4 / 2010$ & 2_ELE270_H & Model $(\mathrm{H})$ at $15 \mathrm{~cm}$ embedment with ELE270 At $100 \%$ intensity \\
\hline 53 & $11 / 4 / 2010$ & 2_TCU_W_H & Model (H) at $15 \mathrm{~cm}$ embedment with TCUW At $100 \%$ intensity \\
\hline 54 & $11 / 4 / 2010$ & 2_TCU_N_H & Model (H) at $15 \mathrm{~cm}$ embedment with TCUN At $100 \%$ intensity \\
\hline 55 & $11 / 4 / 2010$ & 2_CPM000_H & Model $(\mathrm{H})$ at $15 \mathrm{~cm}$ embedment with CPM000 At $100 \%$ intensity \\
\hline
\end{tabular}




\begin{tabular}{|c|c|c|c|}
\hline \# & Date & Test_ID & Test Description \\
\hline 56 & $11 / 4 / 2010$ & 2_CPM090_H & Model $(\mathrm{H})$ at $15 \mathrm{~cm}$ embedment with CPM090 At $100 \%$ intensity \\
\hline 57 & $11 / 4 / 2010$ & 2_LGP000_H & Model (H) at $15 \mathrm{~cm}$ embedment with LGP000 At $100 \%$ intensity \\
\hline 58 & $11 / 4 / 2010$ & 2_LGP090_H & Model (H) at $15 \mathrm{~cm}$ embedment with LGP090 At $100 \%$ intensity \\
\hline 59 & $11 / 4 / 2010$ & 2_L09000_H & Model $(\mathrm{H})$ at $15 \mathrm{~cm}$ embedment with L09000 At $100 \%$ intensity \\
\hline 60 & $11 / 4 / 2010$ & 2_L09090_H & Model (H) at $15 \mathrm{~cm}$ embedment with L09090 At 100\% intensity \\
\hline 61 & $11 / 4 / 2010$ & 2_IPV045_H & Model (H) at $15 \mathrm{~cm}$ embedment with IPV045 At 100\% intensity \\
\hline 62 & $11 / 4 / 2010$ & 2_IPV134_H & Model (H) at $15 \mathrm{~cm}$ embedment with IPV134 At 100\% intensity \\
\hline 63 & $11 / 4 / 2010$ & 2_JOS000_L & Model (L) at $15 \mathrm{~cm}$ embedment with JOS000 At $100 \%$ intensity \\
\hline 64 & $11 / 4 / 2010$ & 2_JOS090_L & Model (L) at $15 \mathrm{~cm}$ embedment with JOS090 At $100 \%$ intensity \\
\hline 65 & $11 / 4 / 2010$ & 2_ELE180_L & Model (L) at $15 \mathrm{~cm}$ embedment with ELE180 At $100 \%$ intensity \\
\hline 66 & $11 / 4 / 2010$ & 2_ELE270_L & Model (L) at $15 \mathrm{~cm}$ embedment with ELE270 At $100 \%$ intensity \\
\hline 67 & $11 / 4 / 2010$ & 2_TCU_W_L & Model (L) at $15 \mathrm{~cm}$ embedment with TCUW At $100 \%$ intensity \\
\hline 68 & $11 / 4 / 2010$ & 2_TCU_N_L & Model (L) at $15 \mathrm{~cm}$ embedment with TCUN At $100 \%$ intensity \\
\hline 69 & $11 / 4 / 2010$ & 2_CPM000_L & Model (L) at $15 \mathrm{~cm}$ embedment with CPM000 At $100 \%$ intensity \\
\hline 70 & $11 / 4 / 2010$ & 2_CPM090_L & Model (L) at $15 \mathrm{~cm}$ embedment with CPM090 At 100\% intensity \\
\hline 71 & $11 / 4 / 2010$ & 2_LGP000_L & Model (L) at $15 \mathrm{~cm}$ embedment with LGP000 At $100 \%$ intensity \\
\hline 72 & $11 / 4 / 2010$ & 2_LGP090_L & Model (L) at $15 \mathrm{~cm}$ embedment with LGP090 At $100 \%$ intensity \\
\hline 73 & $11 / 4 / 2010$ & 2_L09000_L & Model (L) at $15 \mathrm{~cm}$ embedment with L09000 At $100 \%$ intensity \\
\hline 74 & $11 / 4 / 2010$ & 2_L09090_L & Model (L) at $15 \mathrm{~cm}$ embedment with L09090 At $100 \%$ intensity \\
\hline 75 & $11 / 4 / 2010$ & 2_IPV045_L & Model (L) at $15 \mathrm{~cm}$ embedment with IPV045 At $100 \%$ intensity \\
\hline 76 & $11 / 4 / 2010$ & 2_IPV134_L & Model (L) at $15 \mathrm{~cm}$ embedment with IPV134 At $100 \%$ intensity \\
\hline 77 & $12 / 2 / 2010$ & 3_JOSO00_H & Model $(\mathrm{H})$ at $30 \mathrm{~cm}$ embedment with JOS000 At $100 \%$ intensity \\
\hline 78 & $12 / 2 / 2010$ & 3_JOS090_H & Model $(\mathrm{H})$ at $30 \mathrm{~cm}$ embedment with JOS090 At $100 \%$ intensity \\
\hline 79 & $12 / 2 / 2010$ & 3_ELE180_H & Model $(\mathrm{H})$ at $30 \mathrm{~cm}$ embedment with ELE180 At $100 \%$ intensity \\
\hline 80 & $12 / 2 / 2010$ & 3_ELE270_H & Model $(\mathrm{H})$ at $30 \mathrm{~cm}$ embedment with ELE270 At $100 \%$ intensity \\
\hline 81 & $12 / 2 / 2010$ & 3_TCU_W_H & Model $(\mathrm{H})$ at $30 \mathrm{~cm}$ embedment with TCUW At $100 \%$ intensity \\
\hline 82 & $12 / 2 / 2010$ & 3_TCU_N_H & Model (H) at $30 \mathrm{~cm}$ embedment with TCUN At $100 \%$ intensity \\
\hline 83 & $12 / 2 / 2010$ & 3_CPM000_H & Model (H) at $30 \mathrm{~cm}$ embedment with CPM000 At $100 \%$ intensity \\
\hline 84 & $12 / 2 / 2010$ & 3_CPM090_H & Model (H) at $30 \mathrm{~cm}$ embedment with CPM090 At $100 \%$ intensity \\
\hline 85 & $12 / 2 / 2010$ & 3_LGP000_H & Model $(\mathrm{H})$ at $30 \mathrm{~cm}$ embedment with LGP000 At $100 \%$ intensity \\
\hline 86 & $12 / 2 / 2010$ & 3_LGP090_H & Model (H) at $30 \mathrm{~cm}$ embedment with LGP090 At $100 \%$ intensity \\
\hline 87 & $12 / 2 / 2010$ & 3_L09000_H & Model (H) at $30 \mathrm{~cm}$ embedment with L09000 At 100\% intensity \\
\hline 88 & $12 / 2 / 2010$ & 3_L09090_H & Model (H) at $30 \mathrm{~cm}$ embedment with L09090 At 100\% intensity \\
\hline 89 & $12 / 2 / 2010$ & 3_IPV045_H & Model (H) at $30 \mathrm{~cm}$ embedment with IPV045 At 100\% intensity \\
\hline 90 & $12 / 2 / 2010$ & 3_IPV134_H & Model (H) at $30 \mathrm{~cm}$ embedment with IPV134 At 100\% intensity \\
\hline 91 & $12 / 2 / 2010$ & 3_JOS000_L & Model (L) at $30 \mathrm{~cm}$ embedment with JOS000 At $100 \%$ intensity \\
\hline 92 & $12 / 2 / 2010$ & 3_JOS090_L & Model (L) at $30 \mathrm{~cm}$ embedment with JOS090 At $100 \%$ intensity \\
\hline 93 & $12 / 2 / 2010$ & 3_ELE180_L & Model (L) at $30 \mathrm{~cm}$ embedment with ELE180 At $100 \%$ intensity \\
\hline 94 & $12 / 2 / 2010$ & 3_ELE270_L & Model (L) at $30 \mathrm{~cm}$ embedment with ELE270 At $100 \%$ intensity \\
\hline 95 & $12 / 2 / 2010$ & 3_TCU_W_L & Model (L) at $30 \mathrm{~cm}$ embedment with TCUW At $100 \%$ intensity \\
\hline
\end{tabular}




\begin{tabular}{|c|c|l|l|}
$\#$ & Date & Test_ID & \multicolumn{1}{c|}{ Test Description } \\
\hline 96 & $12 / 2 / 2010$ & 3_TCU_N_L & Model (L) at $30 \mathrm{~cm}$ embedment with TCUN At $100 \%$ intensity \\
\hline 97 & $12 / 2 / 2010$ & 3_CPM000_L & Model (L) at $30 \mathrm{~cm}$ embedment with CPM000 At $100 \%$ intensity \\
\hline 98 & $12 / 2 / 2010$ & 3_CPM090_L & Model (L) at $30 \mathrm{~cm}$ embedment with CPM090 At $100 \%$ intensity \\
\hline 99 & $12 / 2 / 2010$ & 3_LGP000_L & Model (L) at $30 \mathrm{~cm}$ embedment with LGP000 At 100\% intensity \\
\hline 100 & $12 / 2 / 2010$ & 3_LGP090_L & Model (L) at $30 \mathrm{~cm}$ embedment with LGP090 At $100 \%$ intensity \\
\hline 101 & $12 / 2 / 2010$ & 3_L09000_L & Model (L) at $30 \mathrm{~cm}$ embedment with L09000 At $100 \%$ intensity \\
\hline 102 & $12 / 2 / 2010$ & 3_L09090_L & Model (L) at $30 \mathrm{~cm}$ embedment with L09090 At $100 \%$ intensity \\
\hline 103 & $12 / 2 / 2010$ & 3_IPV045_L & Model (L) at $30 \mathrm{~cm}$ embedment with IPV045 At $100 \%$ intensity \\
\hline 104 & $12 / 2 / 2010$ & 3_IPV134_L & Model (L) at $30 \mathrm{~cm}$ embedment with IPV134 At $100 \%$ intensity \\
\hline
\end{tabular}




\section{CHAPTER 6-EXPERIMENTAL TESTING RESULTS}

\subsection{Introduction}

This chapter includes test results from Phase I small shake table tests and Phase II T-bar tests, hammer blow tests, and shake table testing. In addition, empirical results are presented to augment Stewart et al (1999b)'s observation on inertial interaction effects for foundation embedment during soil-foundation-structure-interaction.

\subsection{Phase I Results: Fixed Based Performance}

The scale model was fixed on the small shake table and tested to determine the appropriate natural period of vibration. Based on the initial assumption of $5 \%$ overall system damping and the equations presented in Chapter 4, an initial height and weight setup was used to determine the SDOF natural period analytically. For each shake table test, acceleration data from the lumped mass was recorded and plotted against the driving frequency of the shake table input. The natural period of vibration was inferred from the plot at the corresponding peak acceleration as seen in Figure 6-1. In this case, a sample test using a $9.07 \mathrm{~kg}$ lumped mass and a $50 \mathrm{~cm}$ height was used.

A $9.07 \mathrm{~kg}$ lumped mass setup using three weight plates, which consists of one 10 lb. and two 5 lb. steel weights, was selected after trial testing. Series of tests with different height setups were conducted to approximate the target prototype natural periods of vibration of $1.5 \mathrm{~s}$ and $2.5 \mathrm{~s}$. The first mode natural period of vibration from these tests was converted into prototype period by applying similitude analysis with a geometric factor of 10 , and the results were plotted against the corresponding height 
setup in Figure 6-2. Using this plot as a guideline, additional shake table tests were performed to confirm the final lumped mass setups.

As mentioned previously, Model $(\mathrm{H})$ denotes the setup with $72 \mathrm{~cm}$ height that yields a corresponding $2.5 \mathrm{~s}$ natural period for the prototype, and Model (L) symbolizes the setup with $52 \mathrm{~cm}$ height that results in a $1.5 \mathrm{~s}$ natural period for the prototype. The final scale model setups are presented in Table $6-1$.

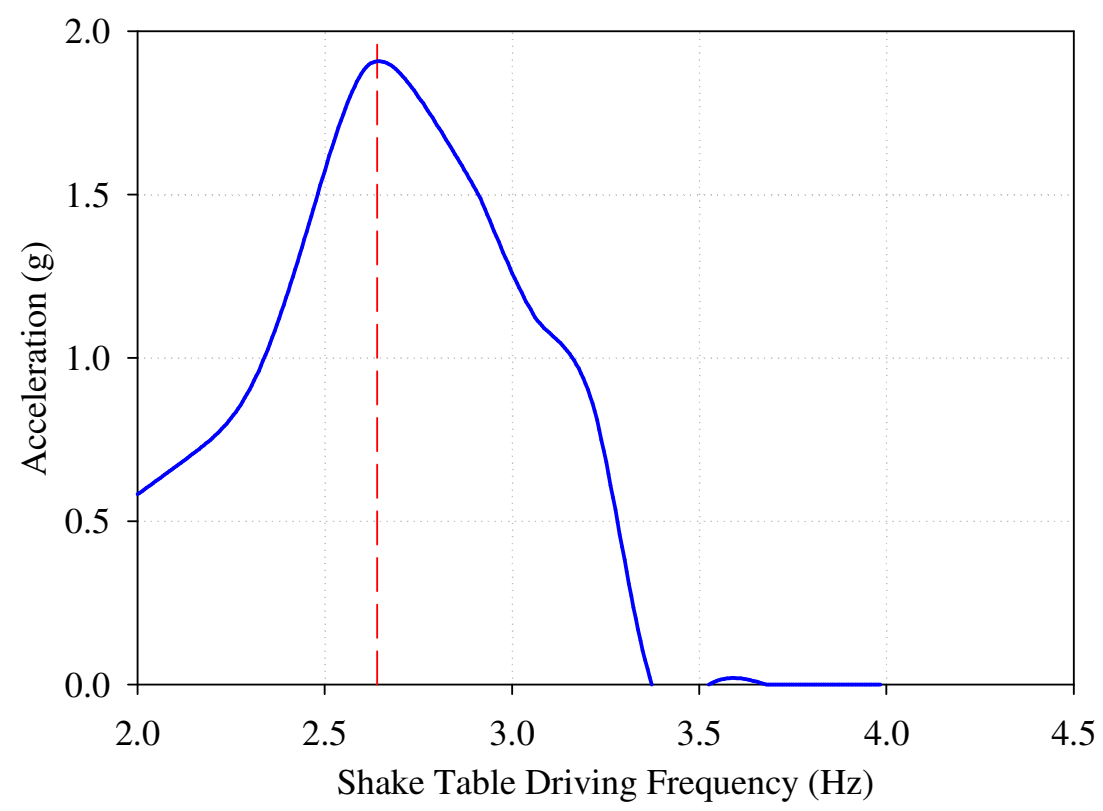

Figure 6-1 Acceleration vs. driving frequency plot of the fixed base small shake table test to determine the first natural mode of vibration of the SDOF model. The model has a fundamental frequency of $2.6 \mathrm{~Hz}$, which also represents a natural period of vibration of $0.38 \mathrm{~s}$ for the model and $1.2 \mathrm{~s}$ for the prototype after similitude analysis 


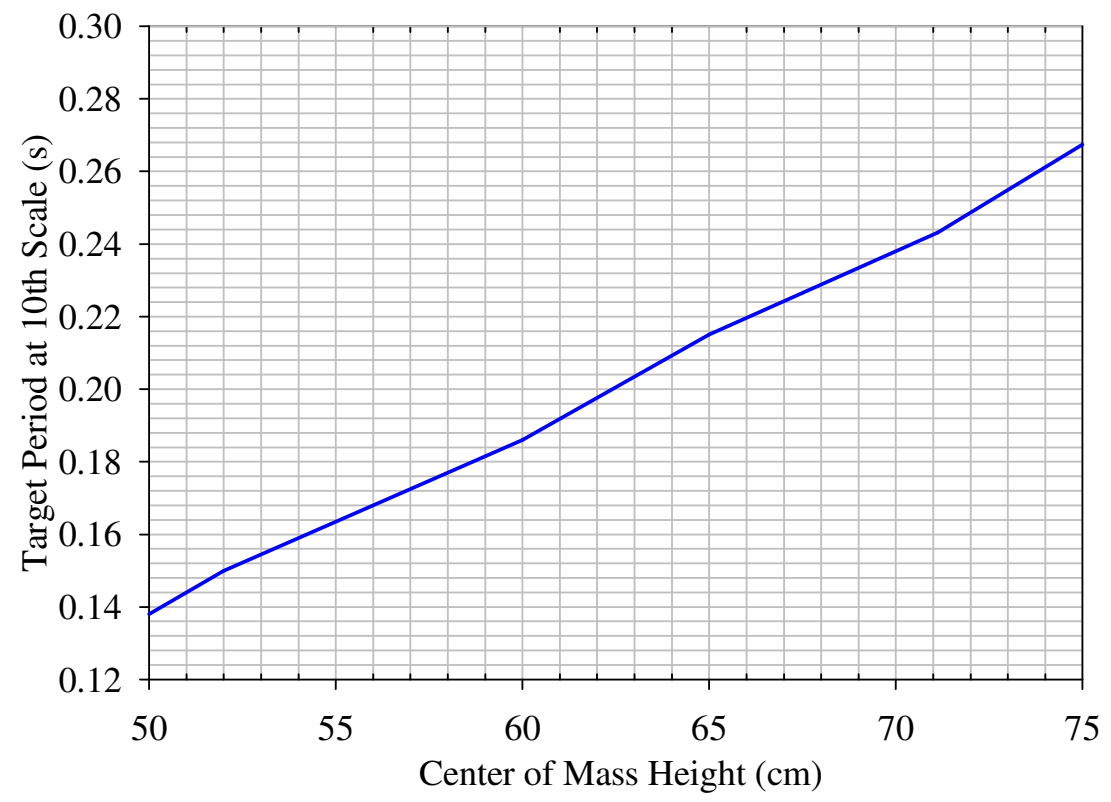

Figure 6-2 Converted prototype period versus the model lumped mass height

Table 6-1 Final setups for the SDOF lollipop of the two target prototypes

\begin{tabular}{|l|c|c|}
\hline ID & Model $(\mathrm{H})$ & Model (L) \\
\hline \hline Lumped Mass (Kg) & 9.07 & 9.07 \\
\hline Height $(\mathrm{cm})$ & 72 & 52 \\
\hline Prototype Period (s) & 2.5 & 1.5 \\
\hline Scale Model Frequency at Peak Acceleration (Hz) & 1.26 & 2.11 \\
\hline
\end{tabular}

Empirical results of scale model natural periods of vibration from shake table testing were compared to results obtained from analytical methods using equations presented in Chapter 3, but no significant relations were observed, possibly due to the unaccounted system damping. The exact damping ratio in the scale model could not be determined analytically due to several different factors. Although the foundation was fixed to the table, there were many structural materials in the model that contributed to the overall damping. Energy could dissipate through the connections between the threaded rod to the foundation, to the lumped mass, or to the accelerometer, in addition to 
the intangible internal friction and viscous damping. There could have been elastic hysteric loss of energy in all of the connections. The rail guides could have also added external friction damping as the coupling nut slides against the lubricated rods during testing.

\subsection{Model Soil Testing Results}

\subsubsection{T-Bar Tests}

Four T-bar pullout tests were performed to approximate the undrained shear strength of the model soil column during Phase II testing. Tests were conducted at one month intervals to observe the strength gain over time. A test was conducted prior to each stage of testing at one of the four locations. Test results are shown in Figure 6-3.

The results show that the soil shear strength increases with depth. The few spikes at the bottom of the soil column were caused by the initial acceleration drive from the overhead acceleration; a similar observation was made in Crosariol (2010). The significant decrease of shear strength near the soil surface at about $10 \mathrm{~cm}$ depth was caused by the lack of overburden pressure as the T-bar was being pulled out of the soil.. The four T-bar tests were taken at different locations, and they experienced random spatial variation in the resulting strength. Results show a $16 \%$ strength gain from Stage 1 to 2 , and an $8 \%$ gain from Stage 2 to 3 , but overall the soil strength increases steadily with depth throughout the soil column.

Since the model soil column was composed of reconstituted soil from Crosariol (2010), his soil testing results served as a baseline. A comparison of the averaged T-bar results and the Crosariol Phase II average is shown in Figure 6-4. Based on the results 
from depth $20 \mathrm{~cm}$ to $90 \mathrm{~cm}$, the average strength gain is $30.5 \%$ with a maximum and minimum increase of $45.2 \%$ and $12.3 \%$, respectively. Both averaged T-bar results show a similar trend strength gain with depth. The model soil column had a higher overall shear strength because of lowered water content and higher unit weight.

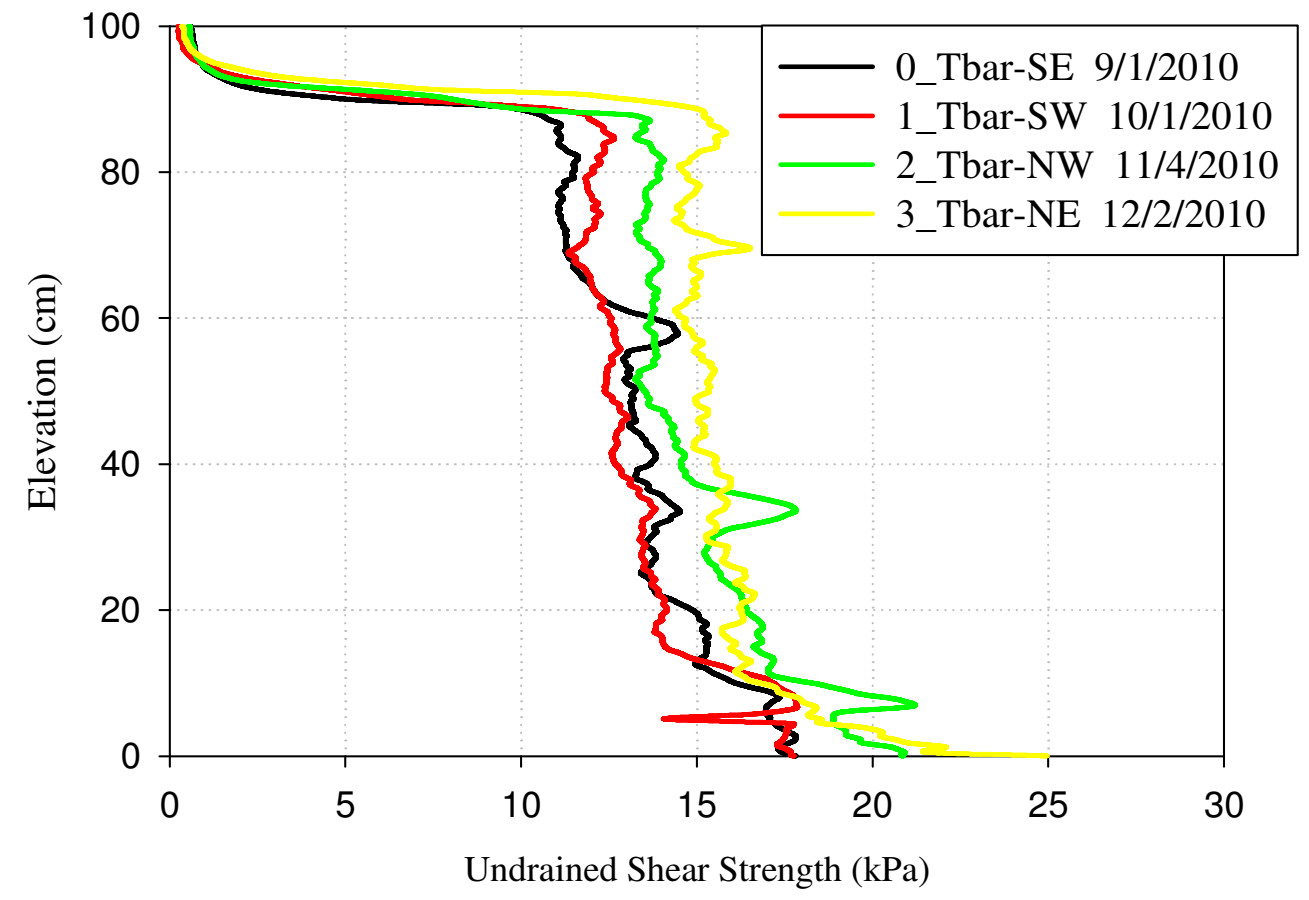

Figure 6-3 T-bar undrained shear strength results comparison of the four trials in Phase II 


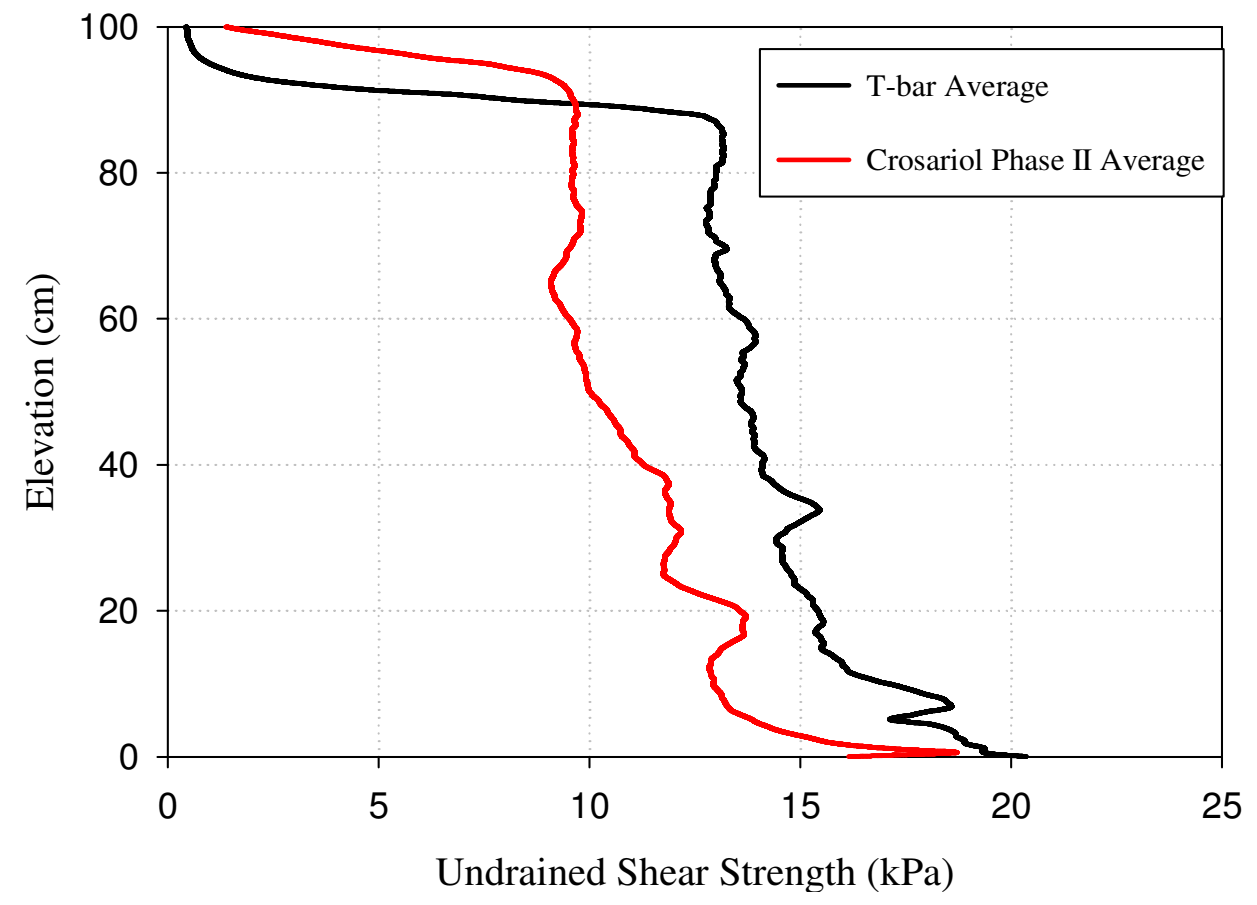

Figure 6-4 Comparison between the average undrained shear strength from the study within and the Crosariol Phase II soil column (after Crosariol 2010)

By using the overhead crane to perform the T-bar pullout test, the overall

undrained shear strength may be overestimated due to the shearing rate effects (Meymand 1998). The overhead crane pulled the T-bar at a rate of $1.29 \mathrm{~cm} / \mathrm{s}$, which could have result in an increase of 30\% to 50\% of peak strength. The T-bar undrained shear strength profiles are coupled with results from the hammer blow tests to approximate the soil shear wave velocity profile in the model soil column.

\subsubsection{Hammer Blow Tests}

Several hammer blow tests, using the top-down approach adopted from Crosariol (2010), were used to approximate the shear wave velocity of the bottom soil column. The tests were conducted for both the free-field and model arrays to check for spatial variation. A total of 20 trials was conducted with 10 for each array with a combination of both east and west directions. The induced wave from the hammer blow propagated down 
the soil column, and the results were recorded from accelerometers in the lower three levels for both arrays.

Figure 6-5 illustrates a typical hammer blow time history plot that is used to approximate the shear wave velocity for the model array. Only the lower three layers of accelerometers (1M, 2M and $3 \mathrm{M})$ were used because of the more distinguishable wave forms as opposed to the high frequency waveform of the upper soil column. The first significant peak in the waveform represents the first arrival of the wave. Knowing the difference in time of first wave arrival and the distance between the subsequent accelerometers, shear wave velocity can be approximated.

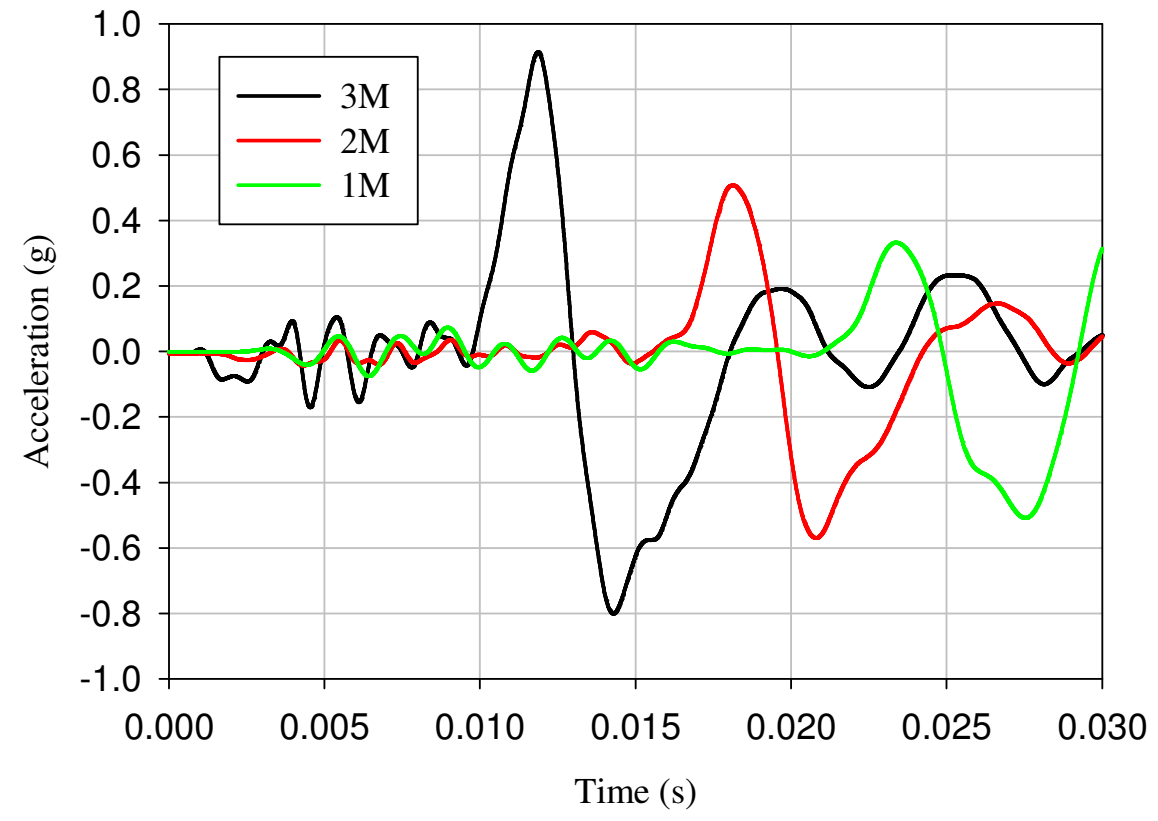

Figure 6-5 Typical wave forms from a top-down hammer blow test for the bottom soil column. Results from MAE5 is shown here

Table 6-2 contains the summary of 18 hammer blow test results. Two of the tests (FFE2 \& MAE4) were defective due to instrumentation signaling and human error, such 
as inaccurate data collection timing and inconsistent blow strength during the testing process. The shear wave velocity results were consistent with a range of 33.38 to 36.29 $\mathrm{m} / \mathrm{s}$ between layers 3 and 2. For the layer between 2 and 1, the overall shear wave velocity increased about $17 \%$ to reach a range of 40.91 to $44.12 \mathrm{~m} / \mathrm{s}$. The average shear wave velocity for layer 3 to 2 and 2 to 1 was 35.1 and $42.3 \mathrm{~m} / \mathrm{s}$ respectively, and the overall average shear wave velocity for the lower soil column was $38.7 \mathrm{~m} / \mathrm{s}$. The hammer blow test resulted in adequate results for the model soil column.

Table 6-2 Summary of hammer blow testing for both arrays including the test ID, shear wave velocity between layer 3 to 2,2 to 1 , and 3 to 1

\begin{tabular}{|c|c|c|c|c|}
\hline & & \multicolumn{3}{|c|}{ Vs (m/s) } \\
Trial \# & Test_ID & 3 to 2 & 2 to 1 & 3 to 1 \\
\hline \hline 1 & FFE1 & 33.58 & 42.45 & 38.02 \\
\hline 2 & FFE3 & 35.71 & 41.67 & 38.69 \\
\hline 3 & FFE4 & 32.61 & 42.45 & 37.53 \\
\hline 4 & FFE5 & 35.71 & 40.91 & 38.31 \\
\hline 5 & FFW1 & 35.16 & 42.45 & 38.80 \\
\hline 6 & FFW2 & 34.09 & 41.67 & 37.88 \\
\hline 7 & FFW3 & 35.71 & 40.91 & 38.31 \\
\hline 8 & FFW4 & 35.16 & 41.67 & 38.41 \\
\hline 9 & FFW5 & 34.77 & 41.85 & 38.31 \\
\hline 10 & MAE1 & 36.29 & 42.45 & 39.37 \\
\hline 11 & MAE2 & 35.71 & 44.12 & 39.92 \\
\hline 12 & MAE3 & 34.62 & 43.27 & 38.94 \\
\hline 13 & MAE5 & 36.29 & 42.45 & 39.37 \\
\hline 14 & MAW1 & 35.71 & 43.27 & 39.49 \\
\hline 15 & MAW2 & 34.62 & 43.27 & 38.94 \\
\hline 16 & MAW3 & 35.71 & 42.45 & 39.08 \\
\hline 17 & MAW4 & 35.16 & 41.67 & 38.41 \\
\hline 18 & MAW5 & 35.16 & 42.45 & 38.80 \\
\hline Average & & $\mathbf{3 5 . 1 0}$ & $\mathbf{4 2 . 3 0}$ & $\mathbf{3 8 . 7 0}$ \\
\hline
\end{tabular}




\subsubsection{Development of Shear Wave Velocity Profile}

Shear wave velocity profile of the soil column is important for seismic soilstructure interaction analysis. By using the soil undrained shear strength profile established from the T-bar tests in conjunction with the shear wave velocity data for the lower soil column, a full shear wave velocity profile of the soil column was developed. Using the relationship shown in Figure 6-6 developed by Dickenson (1994), shear wave velocity was estimated from undrained shear strength. Once shear wave velocity profile was approximated, all the values were scaled and calibrated based on the shear wave velocity obtained from the hammer blow tests for the lower soil column.

Figure 6-7 presents the best estimate shear wave velocity profile superimposes on the scaled and converted shear wave velocity profile from the T-bar pull out and the hammer blow test results. The scaled shear wave velocity values were about $50 \%$ less than the converted undrained shear strength value using the Dickenson relation. Note that the combined calibration method to obtain the shear wave velocity using T-bar pull-out tests and the Dickenson relation tends to overestimate true shear wave velocity by $50 \%$ to 80\% (Crosariol 2010).

Figure 6-8 compares the best estimated shear wave velocity profile of this study

The T-bar pullout tests suggest a higher undrained shear strength in the soil, but that does not guarantee a higher shear wave velocity. The Dickenson relation primarily applies for soil with higher shear strength, so the accuracy of the direct conversion from shear strength to shear wave velocity for an extremely soft soil, such as the model soil used, might not be accurate, and more research is needed to justify the direct conversion method. 


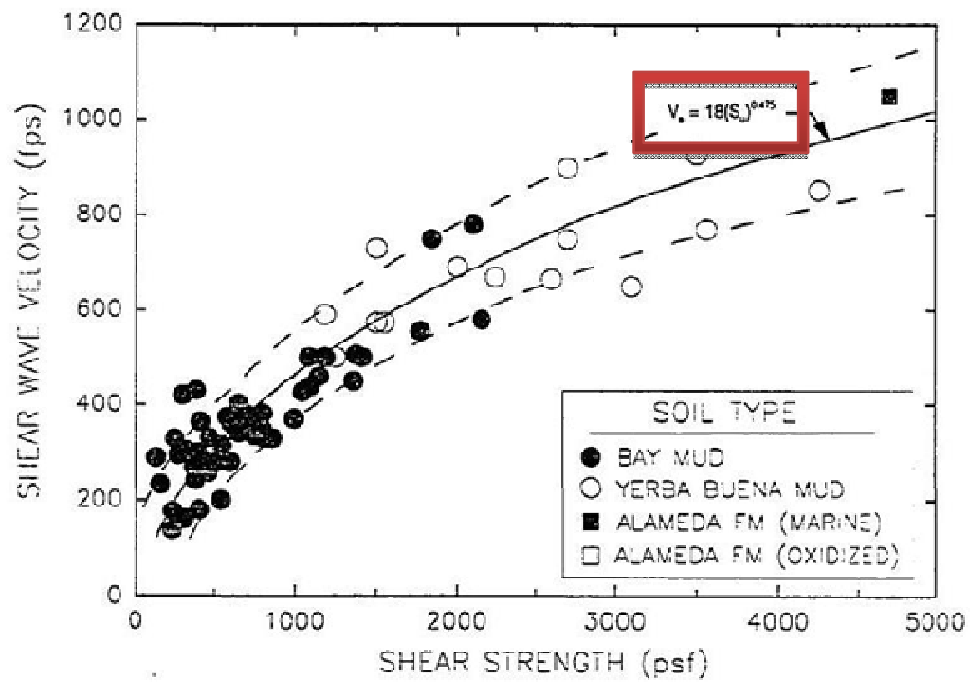

Figure 6-6 Dickenson (1994) relationship that correlates soil shear strength to shear wave velocity for clay soil

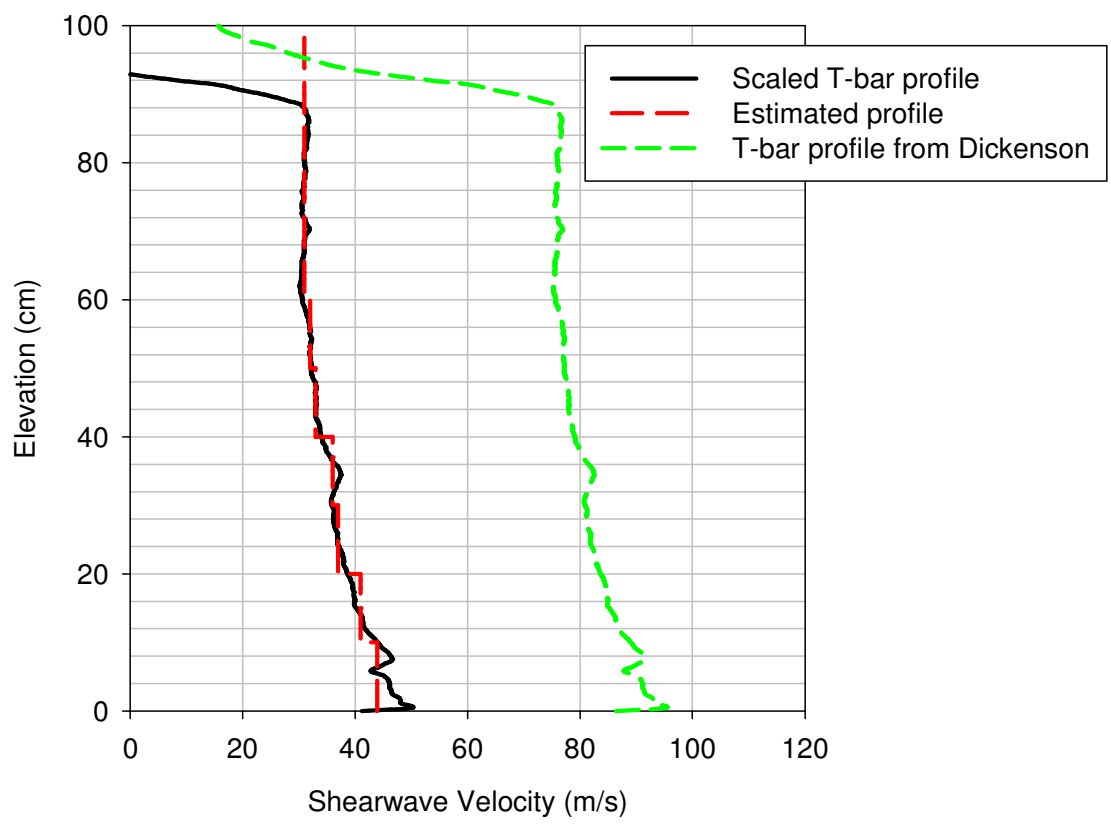

Figure 6-7 Soil shear wave velocity profile interpretation progression: From T-bar profile from undrained shear strength, and scaled shear wave velocity profile using shear wave velocity data from hammer blow test, and the best estimated soil shear wave velocity profile. 


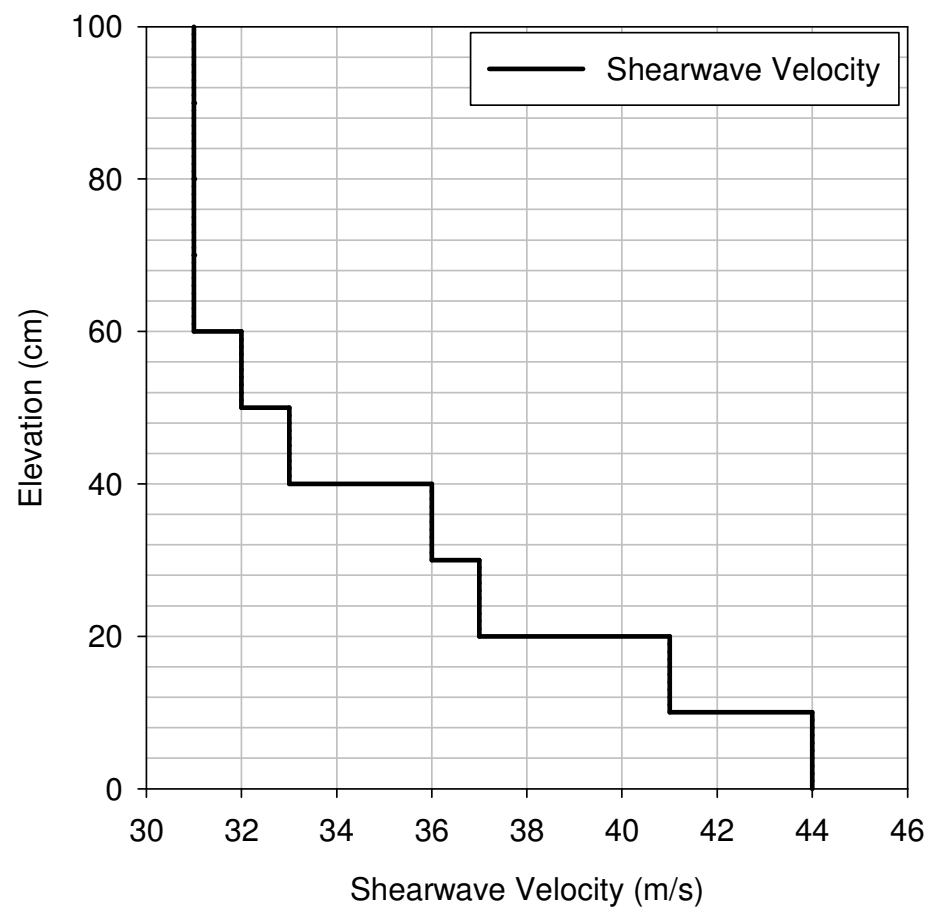

Figure 6-8 best estimated shear wave velocity profile of this study

\subsection{Phase II: Testing Performance}

\subsubsection{Comparison to Recent Tests}

In Phase II of this study, surface free-field array results from accelerometer 5F were compared to Crosariol Phase I free-field study to provide a baseline comparison among the shake table performance, instrumentation calibration, and most importantly, the soil column seismic response. The surface free-field results from the six identical motions used in Crosariol Phase I study and the study within were analyzed using MATLAB code to generate acceleration time histories and 5\% damped response spectra in prototype scale. Times steps from the recorded model scale data were scaled to the prototype scale. The response spectra for the six comparable motions are plotted in Figure 6-9 through Figure 6-11. 
From the spectral response comparison, the predominant resonate period of the model soil column decreased $31 \%$ from an average of 0.51 seconds to an average of 0.35 seconds from Crosariol's results. The decrease of resonate period was caused by the increased model soil stiffness and an associated higher shear wave velocity in the lower column. The stiffer soil amplifies ground motion through the soil column, thus resulting in higher peak spectral acceleration at lower resonate periods at the soil surface for most motions. Although the input motions are identical for both free-field testing phases, the magnitudes of amplification varied due to the nonlinear effects in such soft soil. The surface Chi Chi West motion was conducted at 90\% intensity for Crosariol Phase I testing, therefore, the spectral acceleration was much lower than the study within, and the resonate period remain higher from the less stiff soil column. 

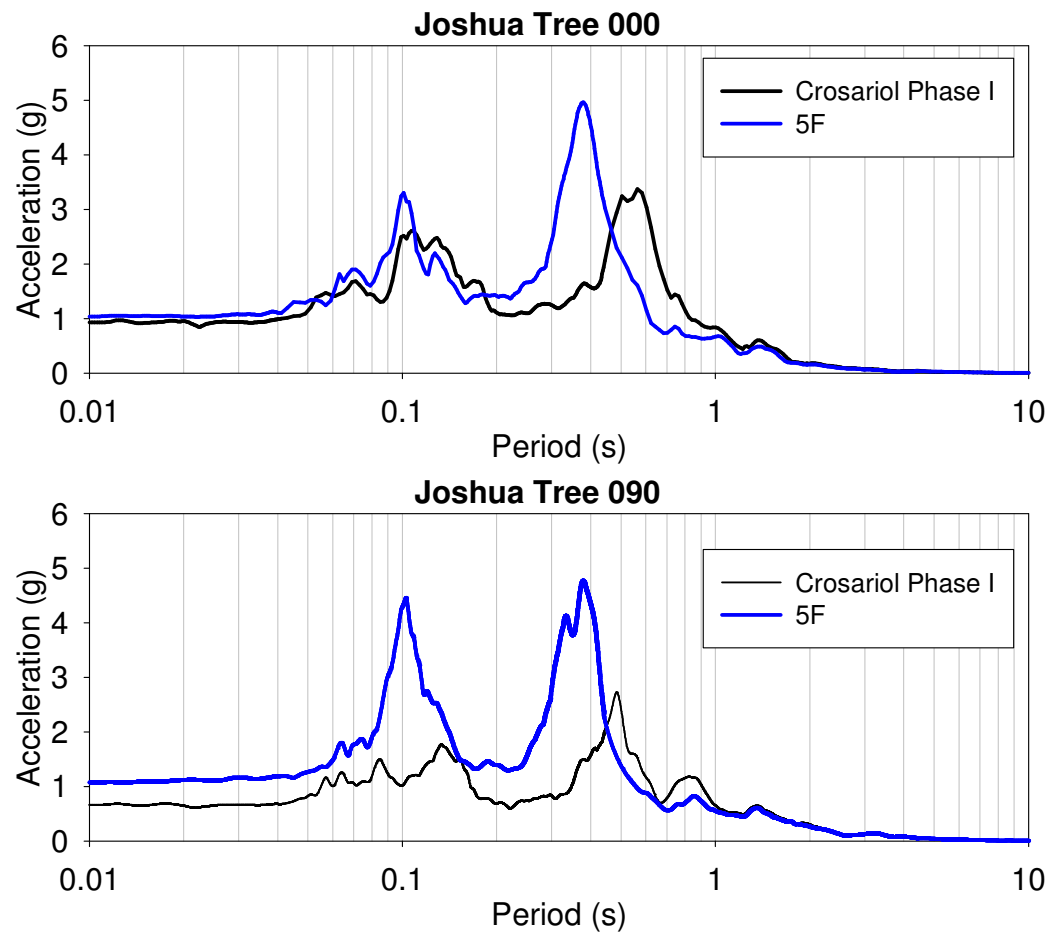

Figure 6-9 5\% damped surface response spectra comparison for the Joshua Tree motions
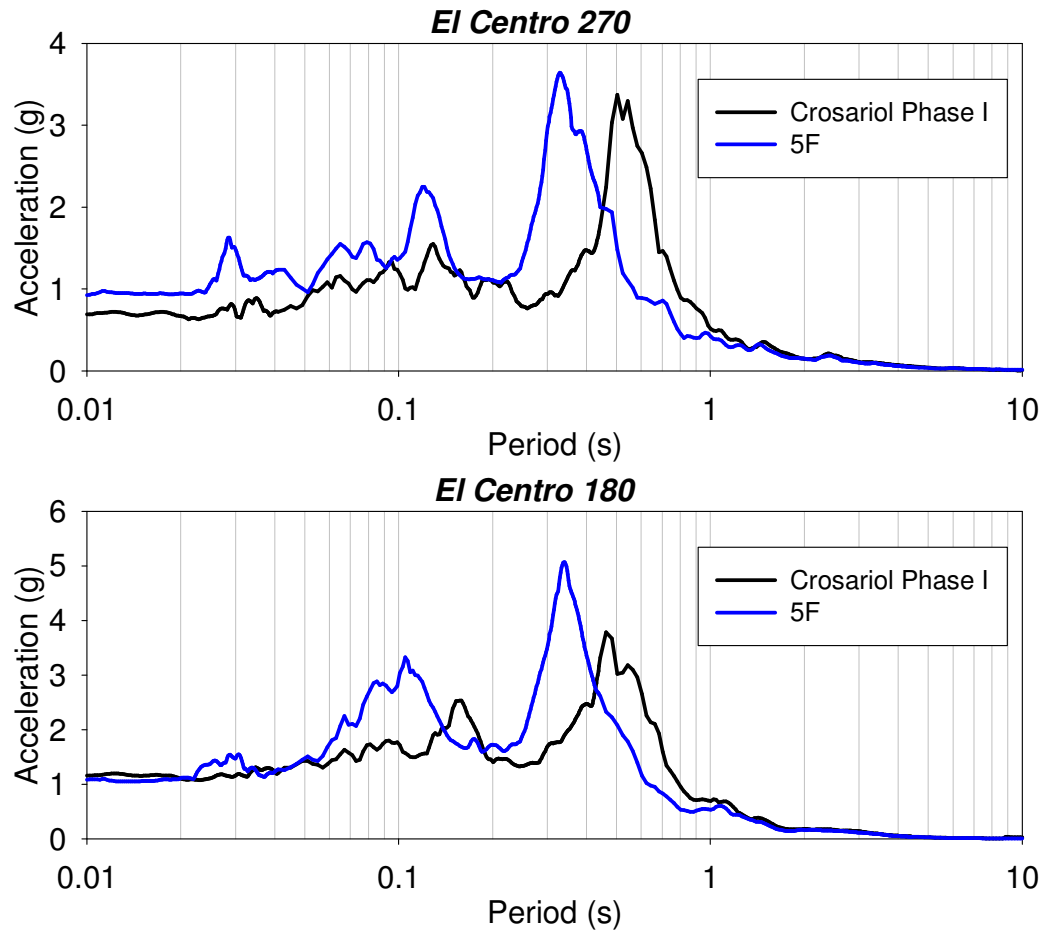

Figure 6-10 5\% damped surface response spectra comparison for the El Centro motions 

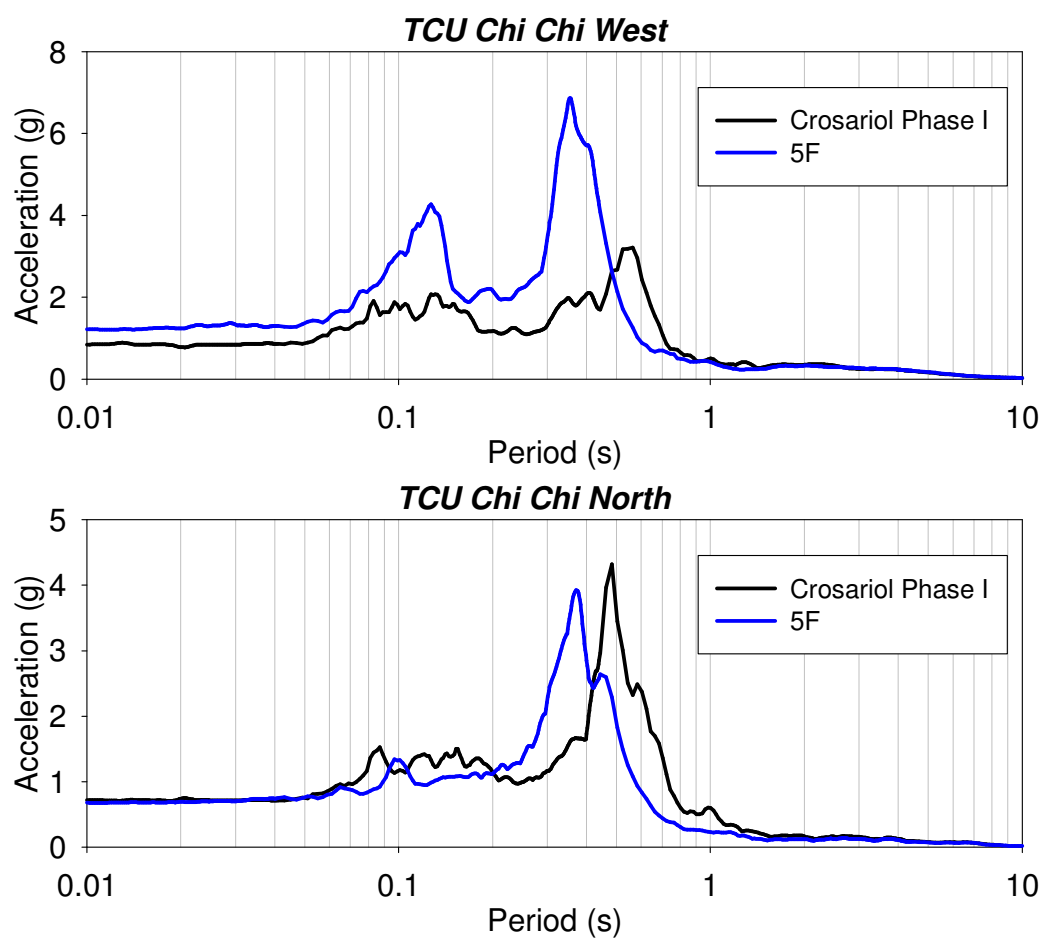

Figure 6-11 5\% damped surface response spectra comparison for the TCU Chi Chi motions

\subsubsection{Displacement Comparison}

Soil column surface and shake table level displacements were recorded during shake table testing to monitor performance coherence in the shake table and testing container. Figure 6-12 compares the displacement data collected from the displacement string pots from the El Centro 180 test. Results show the table and testing container performed in a consistent manner. The relative displacement between the table and the test container was minimal throughout dynamic loading. The same coherent behavior was observed for the other 13 motions used, and corresponding comparison plots are placed in Appendix I. 


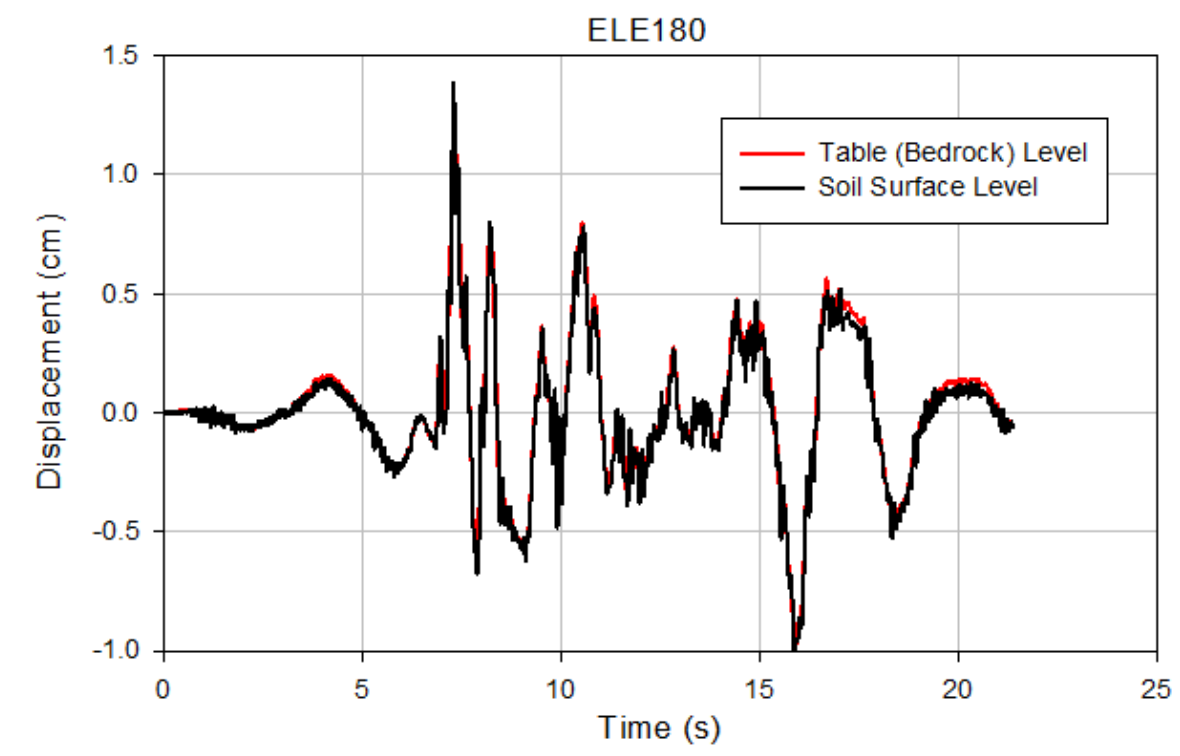

Figure 6-12 Comparison of displacement data from the string pots between the soil surface level and the shake table level for the El Centro 180 motion

\subsection{Soil Column Accelerometer Performance}

The 16 acceleration data array obtained from each test were converted into $5 \%$ damped spectral values at the prototype scale using MATLAB. A total of 1296 arrays of acceleration data were transformed from the 81 serviceable tests. To streamline the result presentation, only data from the El Centro 180 motion, which has the most representative data results, are presented in figures throughout this section for consistency. A table summary containing the pertinent results from each test is presented in each section when necessary.

Figure 6-13 shows all the 5\% damped spectral acceleration time history from the 3_ELC180_H test. The results show the peak spectral accelerations occurred approximately at $0.33 \mathrm{~s}$, with the exception of the lumped mass accelerometer result. There is a consistent trend of peak spectral value amplification with an increase of 
elevation. Similar trend is observed in other tests. A table comprises of pertinent peak spectral acceleration values is included in the summary Section 6.9.

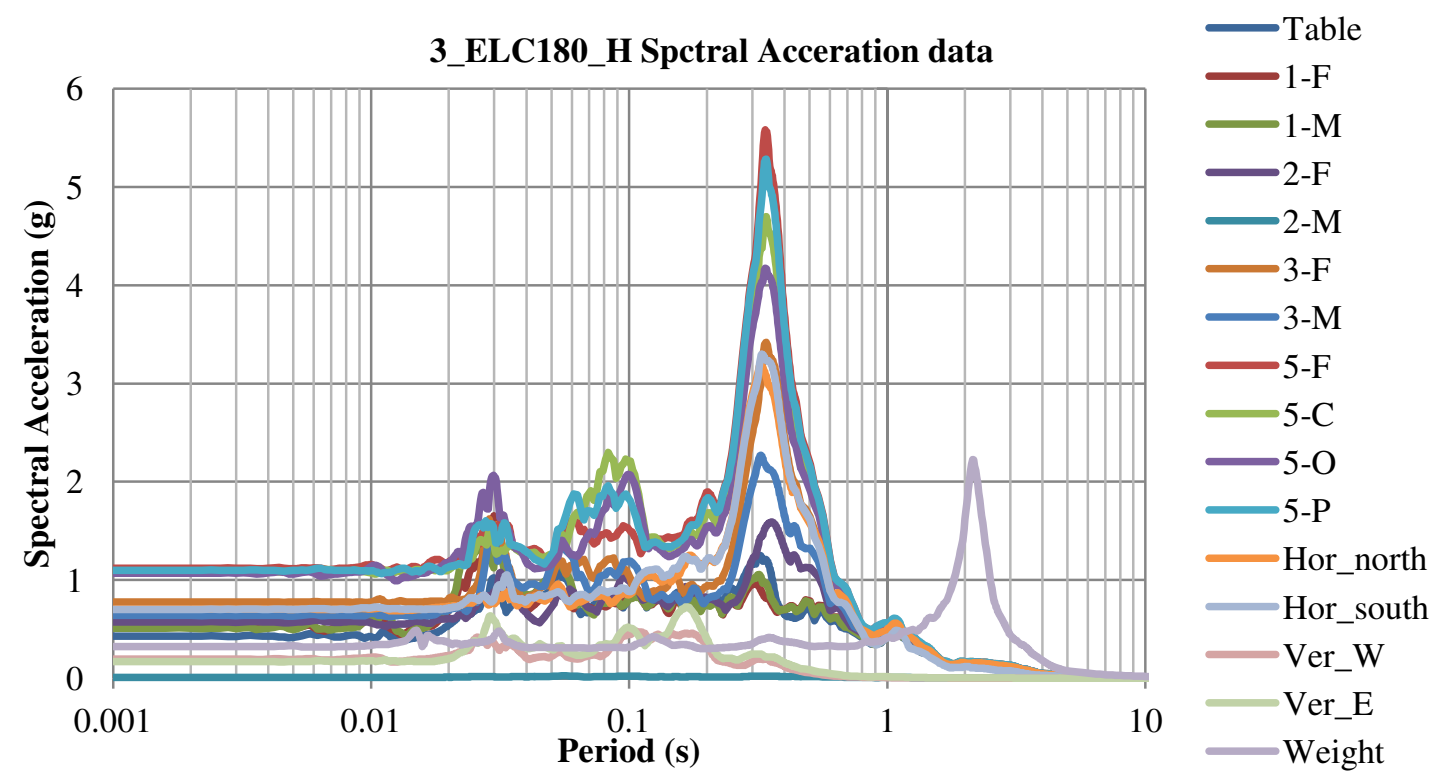

Figure 6-13 5\% damped spectral acceleration time history for all the acceleration data from the 3_ELC180_H test

\subsubsection{Free-field Array vs. Model Array}

Spectral acceleration data for the table, level 1, level 2, and level 3 accelerometers are compared in Figure 6-14. A slight amplification in peak spectral values occur at level 1 and level 2 free-field and model array recordings. However, at level 3, the model array acceleration amplitude at the predominant period was on average $36 \%$ higher than the free-field array for test 1_ElC180_H. Similar spikes in peak spectral acceleration were observed in other testing results. The increase of spectral amplitude at 3F model array accelerometer was caused by the additional boundary effect from the vertical load exerted by the scale model, which resulted in higher shear wave velocity and greater ground motion amplification from the stiffer soil column.

Table 6-3 summarizes the amplification of spectral amplitude in the model array compared to the free-field array at level 3. The Stage 1 results, which were collected 
when the scale model was located $40 \mathrm{~cm}$ above the $3 \mathrm{~F}$ accelerometer, had a $24.5 \%$ difference between 3M and 3F. At Stage 2, where the scale model was embedded by 15 $\mathrm{cm}$, the percent different increase to $27.7 \%$. At Stage 3, where the foundation was only $10 \mathrm{~cm}$ above $3 \mathrm{M}$, the difference increased to $33.2 \%$. This trend shows an increase in $3 \mathrm{M}$ peak spectral amplitude as the foundation embedment increases.

Table 6-4 shows the average percentage difference among the three stages. As expected, the closer the foundation gets to the $3 \mathrm{M}$ accelerometer, the more the differences between $3 \mathrm{~F}$ and $3 \mathrm{M}$ increase. At Stage 3, the $3 \mathrm{M}$ and $3 \mathrm{~F}$ difference increases by $35.7 \%$ when compared to the no-embedment setup at Stage 1 . The accelerometer results show variation in the ground motion in the free-field and model arrays. Although natural variation in the soil can contribute to the differences in amplitude, the predominant influence is from the overbearing pressure from the scale model.

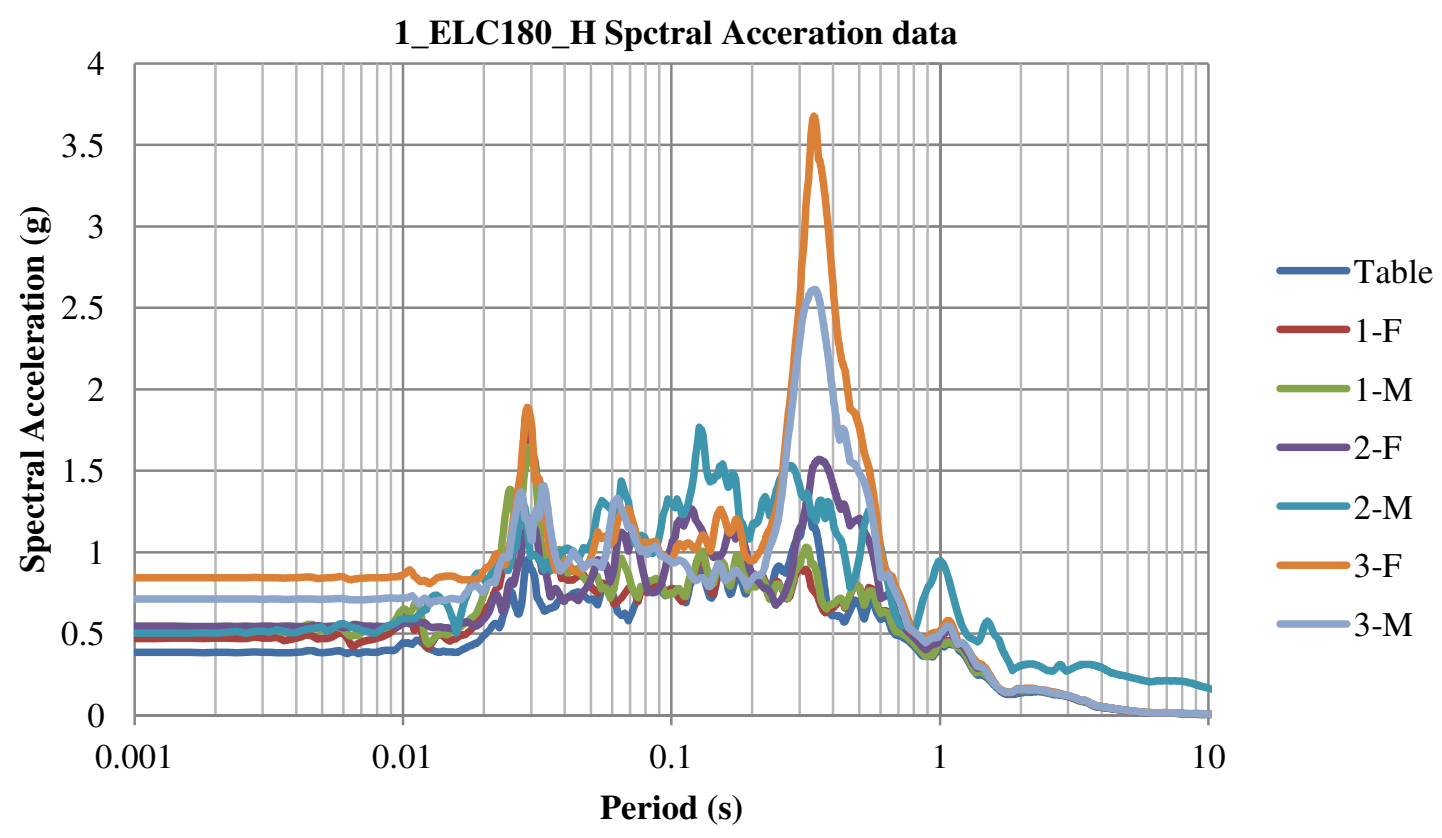

Figure 6-14 5\% damped spectral acceleration time history for the table, level 1, level 2, and level 3 acceleration data from the 1_ELC180_H test 
Table 6-3 Summary of percentage increase in spectral amplitude at the predominant period at the model array compared to the free-field array

\begin{tabular}{clccc}
\hline & & \multicolumn{3}{c}{ Difference for Stage (level 3) } \\
$\#$ & Motion & 1 & 2 & 3 \\
\hline \hline 1 & ELC180 & 36.6 & 42.5 & 50.9 \\
2 & ELC270 & 29.9 & 22.7 & 47.2 \\
3 & JOS000 & 20.7 & 20.2 & 29.0 \\
4 & JOS090 & 27.1 & 27.3 & 34.0 \\
5 & TCU_N & 23.1 & 27.6 & 35.6 \\
6 & TCU_W & 36.1 & 36.4 & 43.4 \\
7 & CPM000 & 11.3 & 27.5 & 9.9 \\
8 & CPM090 & 20.9 & 25.7 & 10.3 \\
9 & LGP000 & 14.4 & 25.0 & 10.8 \\
10 & LGP090 & 22.0 & 17.8 & 23.7 \\
11 & L09000 & 19.0 & 24.0 & 36.4 \\
12 & L09090 & 13.2 & 15.3 & 41.6 \\
13 & IPV045 & 28.9 & 32.2 & 42.1 \\
14 & IPV135 & 39.5 & 43.1 & 50.4 \\
\hline Average & & $\mathbf{2 4 . 5}$ & $\mathbf{2 7 . 7}$ & $\mathbf{3 3 . 2}$ \\
\hline \hline
\end{tabular}

Table 6-4 Average percentage increase of peak spectral value from $3 \mathrm{~F}$ to $3 \mathrm{M}$ between each testing stage

\begin{tabular}{cc}
\hline Stage & \% increase from stage to stage \\
\hline \hline 1 to 2 & 13.0 \\
2 to 3 & 20.1 \\
1 to 3 & 35.7 \\
\hline \hline
\end{tabular}

\subsubsection{Accelerometer Failure}

Accelerometer 2M has irregular data recording for many of the tests. Results from the 2_ELC180_H test are shown in Figure 6-15, which highlights accelerometer 2M's with irregular results. This unwarranted behavior was observed in many other tests, thus making the results from $2 \mathrm{M}$ impractical. However, accelerometer $2 \mathrm{M}$ results were serviceable during the top-down hammer blow test to help identify the shear wave velocity in the lower soil column. 


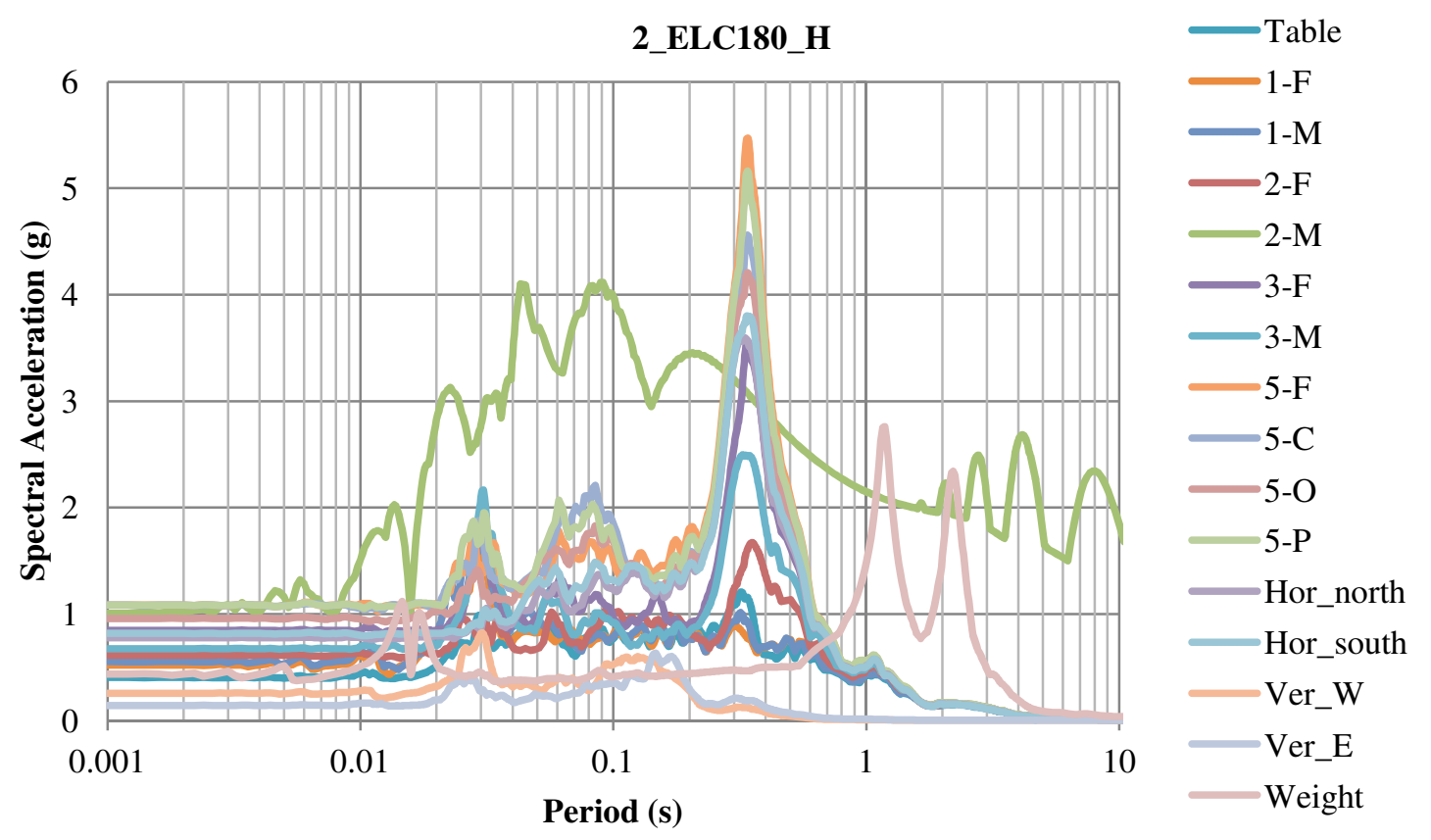

Figure 6-15 5\% damped spectral acceleration time history for all the acceleration data from the 2_ELC180_H test

\subsection{Scale model Accelerometer Performance}

Accelerometer data from LGP000 were used to showcase the representative results in this section. Results for foundation motion coherence, or twisting, foundation rocking, and SDOF setup performance are presented in the following subsections.

\subsubsection{Foundation Horizontal Recording}

Two accelerometers, Hor_south and Hor_north, were mounted equidistance away from the SDOF setup onto the foundation to measure the horizontal acceleration. Results from the two accelerometers were compared to verify motion coherence on the foundation for possible foundation twisting during shake table testing. Figure 6-16 compares the time history results from the two accelerometers, and the results show adequate consistency between the two. In Figure 6-17, the data were converted to 5\% damped spectral response, and the results show similar coherence with an average of 
$4.5 \%$, difference, which is negligible. No reasonable judgment regarding foundation twisting during seismic loading can be made based on empirical observation and data.

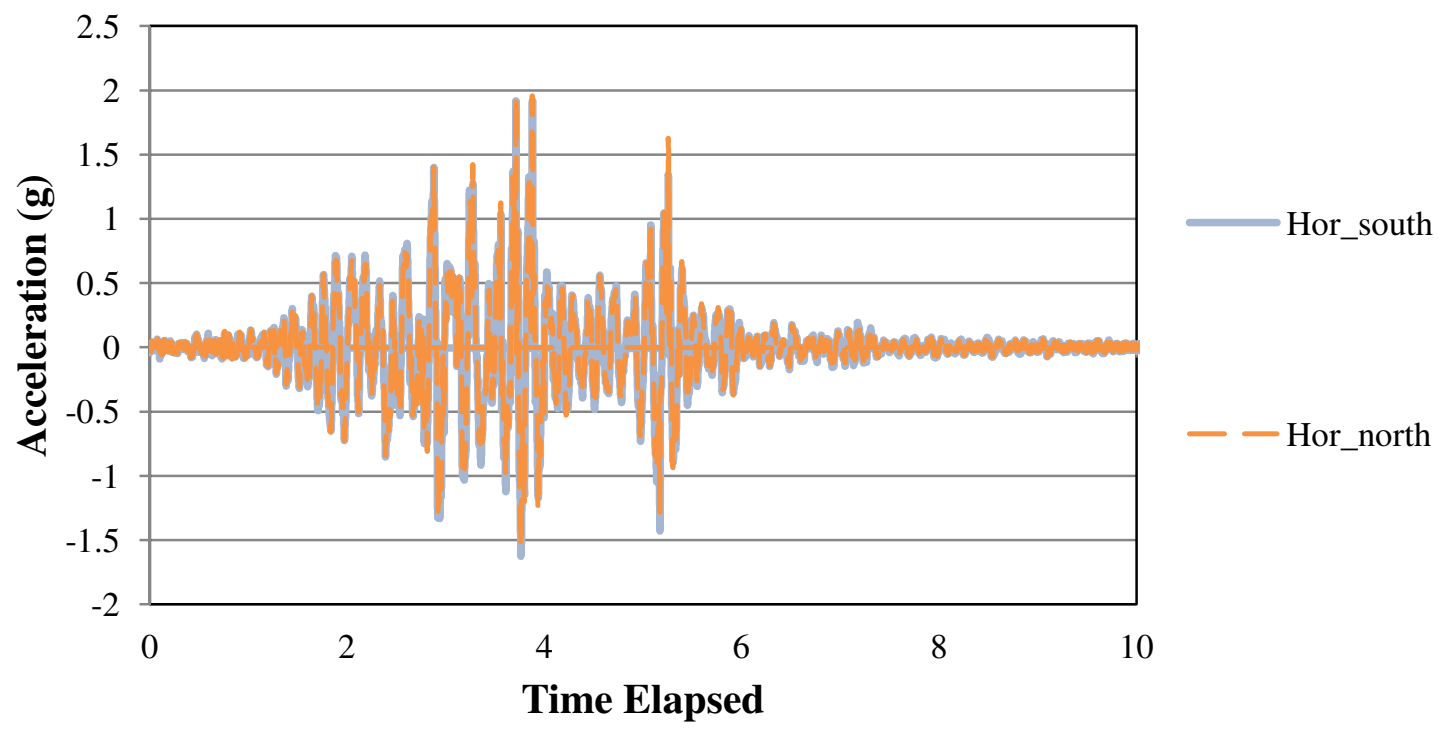

Figure 6-16 Time history comparison for the horizontal accelerometers on the foundation for test 1_LGP000_L

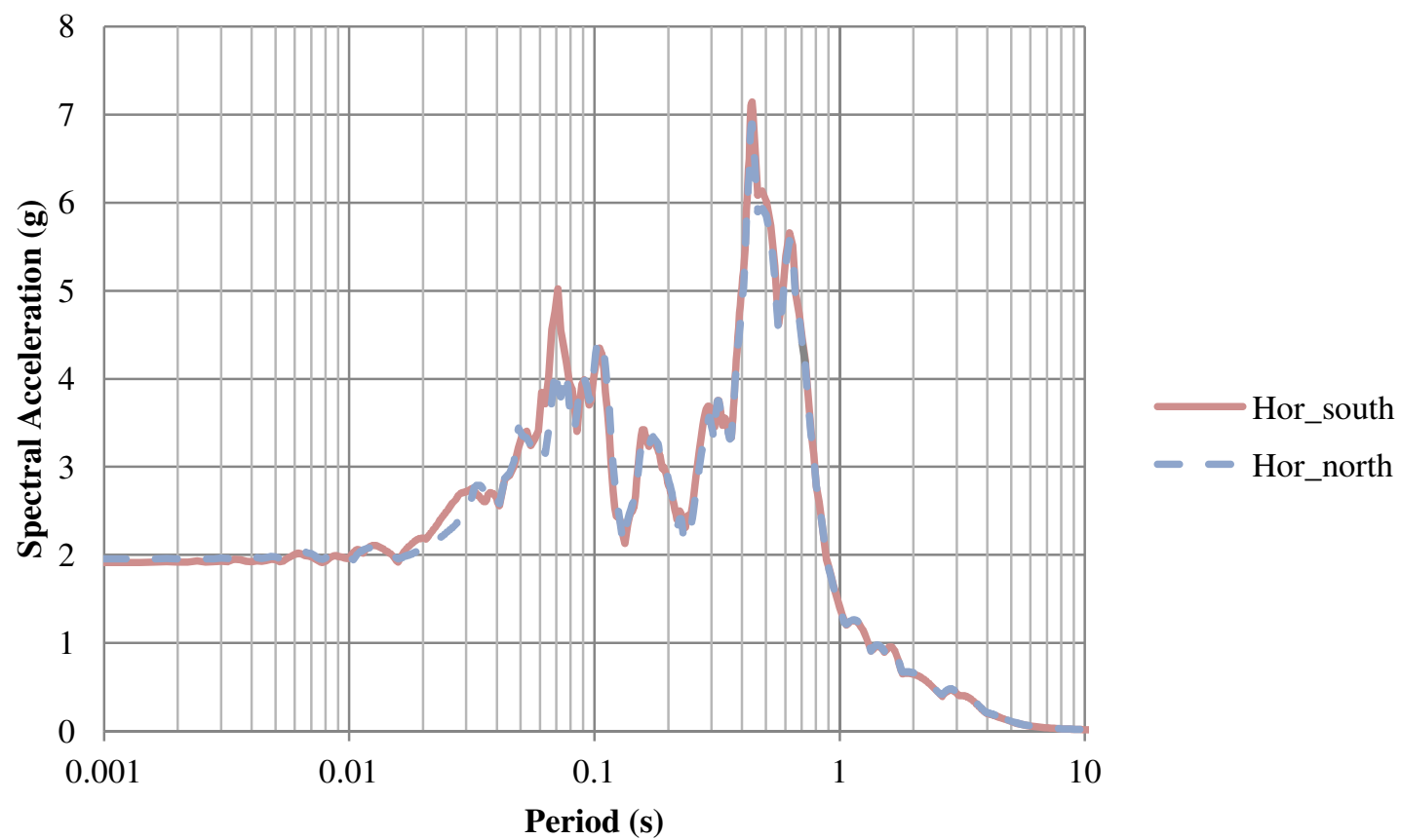

Figure 6-17 5\% damped spectral response on the horizontal accelerometers on the foundation for test 1_LGP000_L 


\subsubsection{Foundation Rocking}

Foundation rocking during seismic loading can influence overall dynamic behavior. Two accelerometers (Ver_East and Ver_West) were installed on the opposite sides of the lumped mass parallel to the shacking direction to collect vertical acceleration data for possibly foundation rocking. The results were compared to determine if there were any "butterfly" effects, which could justify the rocking behavior when the wave forms are recorded in the opposite amplitude at the same time. In Figure 6-18 and 6-19, the spectral results and time history results of the two vertical accelerometers are compared, and no significant sign indicates a definite foundation rocking behavior. Coupled with the displacement results shown earlier, the overall testing container and model soil system behave relatively uniform during shake table testing. No significant foundation rocking behavior was observed, thus eliminating the concern rocking.

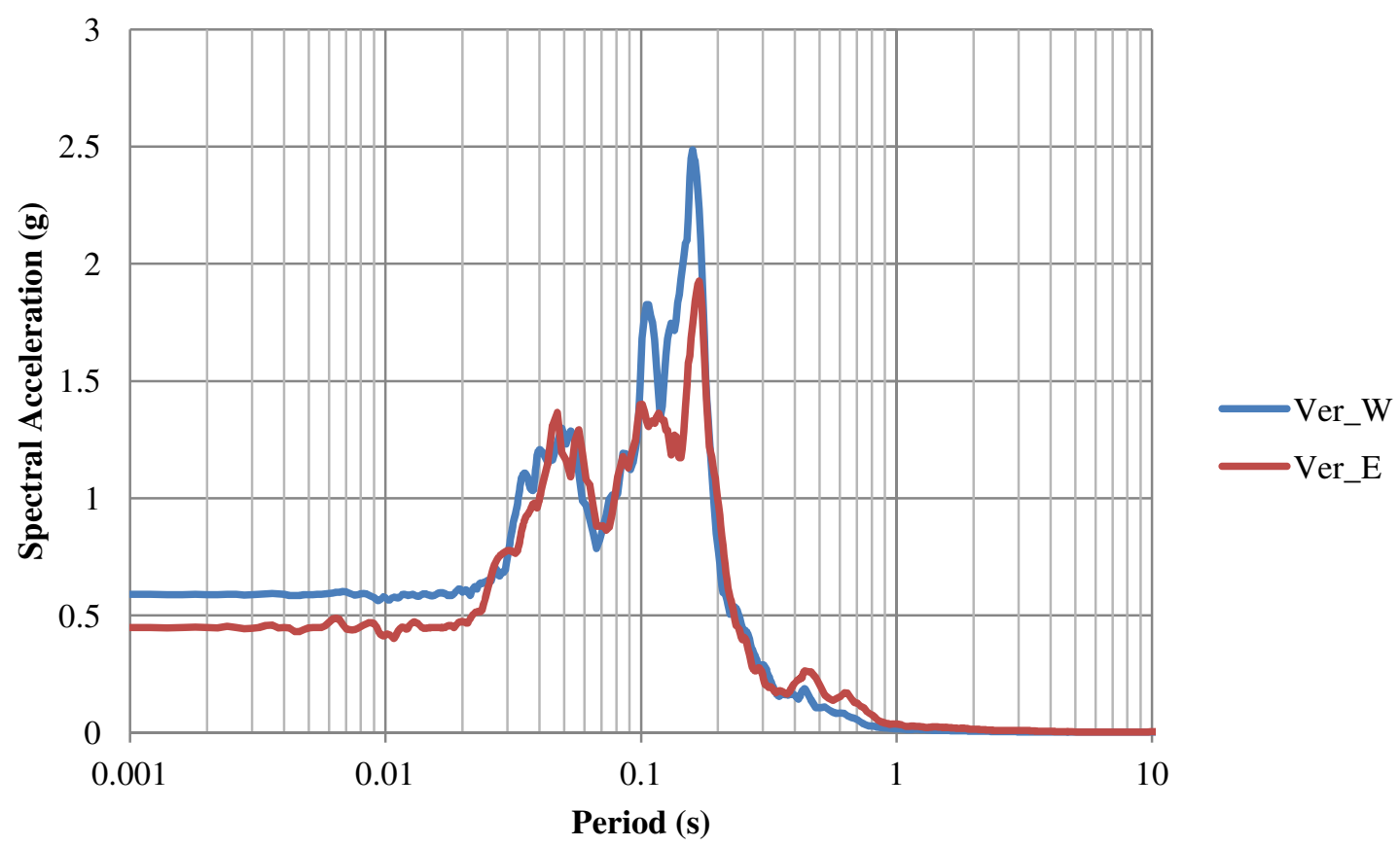

Figure 6-18 5\% damped spectral response on the vertical accelerometers on the foundation for test 3_LGP000_L 


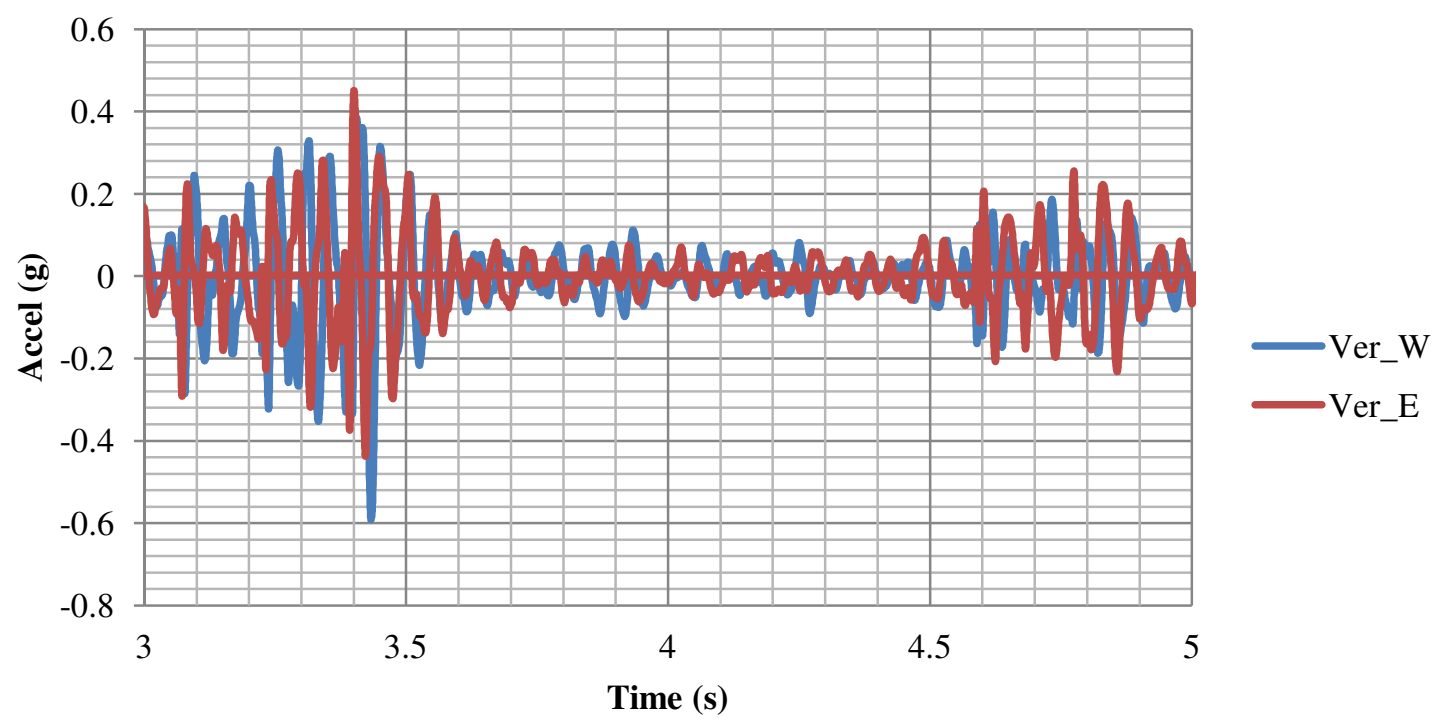

Figure 6-19 Time history comparison for the vertical accelerometers on the foundation for test 3_LGP000_L

\subsubsection{Lumped mass}

Results from the lumped mass accelerometer were converted into 5\% damped spectral response to compare peak spectral amplitudes and corresponding periods. The lumped mass setups, Model High and Model Low, show consistent results in terms of peak spectral periods, but the peak amplitudes varied. No defining correlation between the peak spectral amplitude and the embedment depth of the model foundation was observed as seen in Figure 6-20, which shows six spectral results from lumped mass accelerometer data.

For the other 13 motions, the corresponding periods at the peak spectral accelerations for both the High and Low lumped mass model setups were consistent despite natural random variation, but the peak amplitudes were less uniform. 


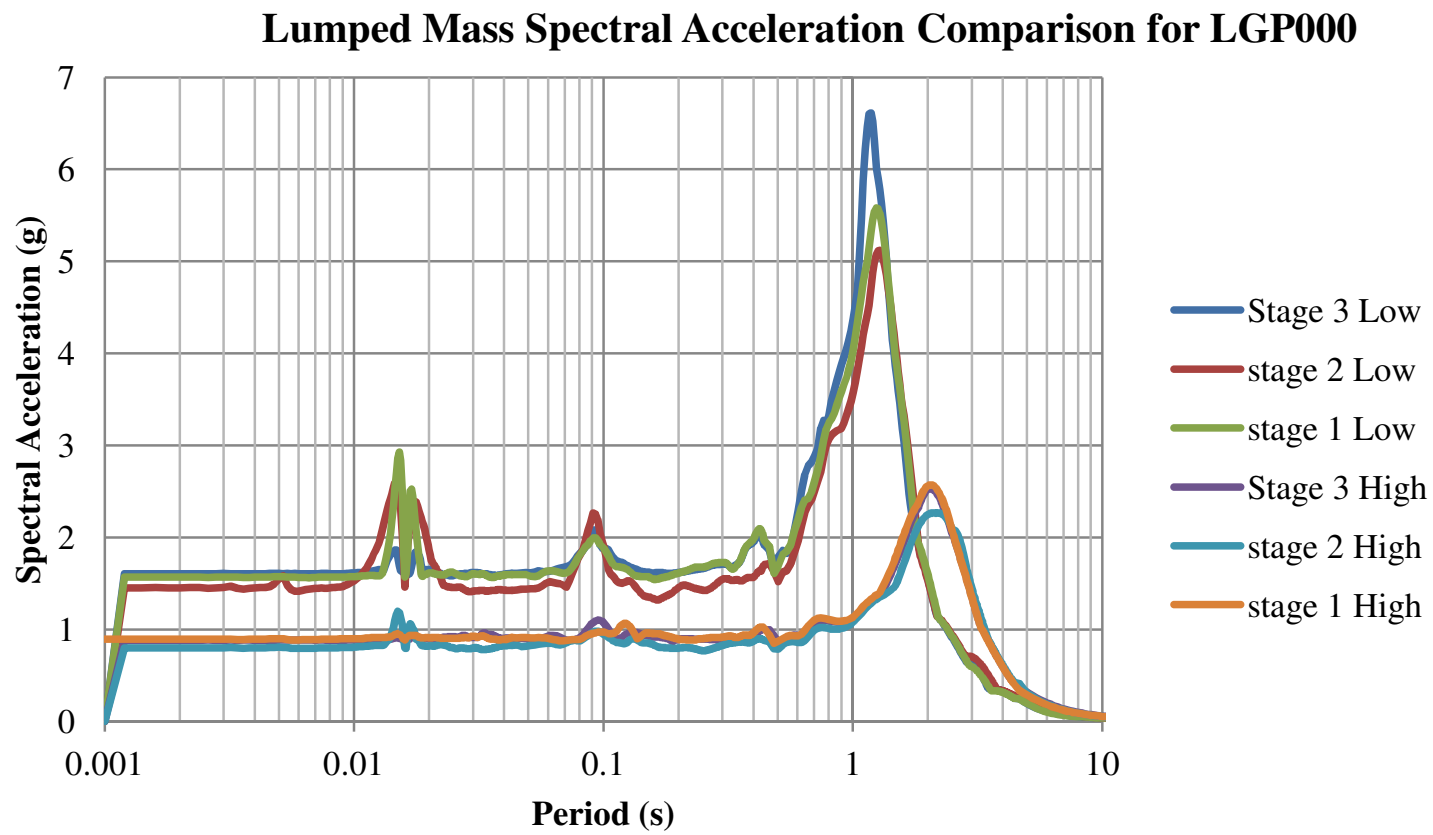

Figure 6-20 5\% damped spectral response comparison for the lumped mass accelerometer for motion LGP000

\subsection{Peak Acceleration Results and Comparison}

\subsubsection{Overview}

Peak acceleration data from the free-field surface accelerometer and the foundation accelerometer are compared in Figure 6-21. A total of 81 data points were plotted. Results are presented in three different stages of testing and separated into the Model High and Model Low forms.

As expected, the foundation level accelerations tended to be less than the freefield surface accelerations. The percentage difference for stage 1 was $19 \%$, for stage 2 was $18.1 \%$, and stage 3 was significantly higher at $31.7 \%$. Stage 2 had an embedment ratio of 0.58 , and the results of percentage differences between the free-field and foundation level were similar to stage 1, which had no embedment. 
Comparisons to Stewart (1998) empirical observation results are shown in the following section. Additional discussions of embedment depth and foundation period influence on the peak ground acceleration are also presented in following sections.

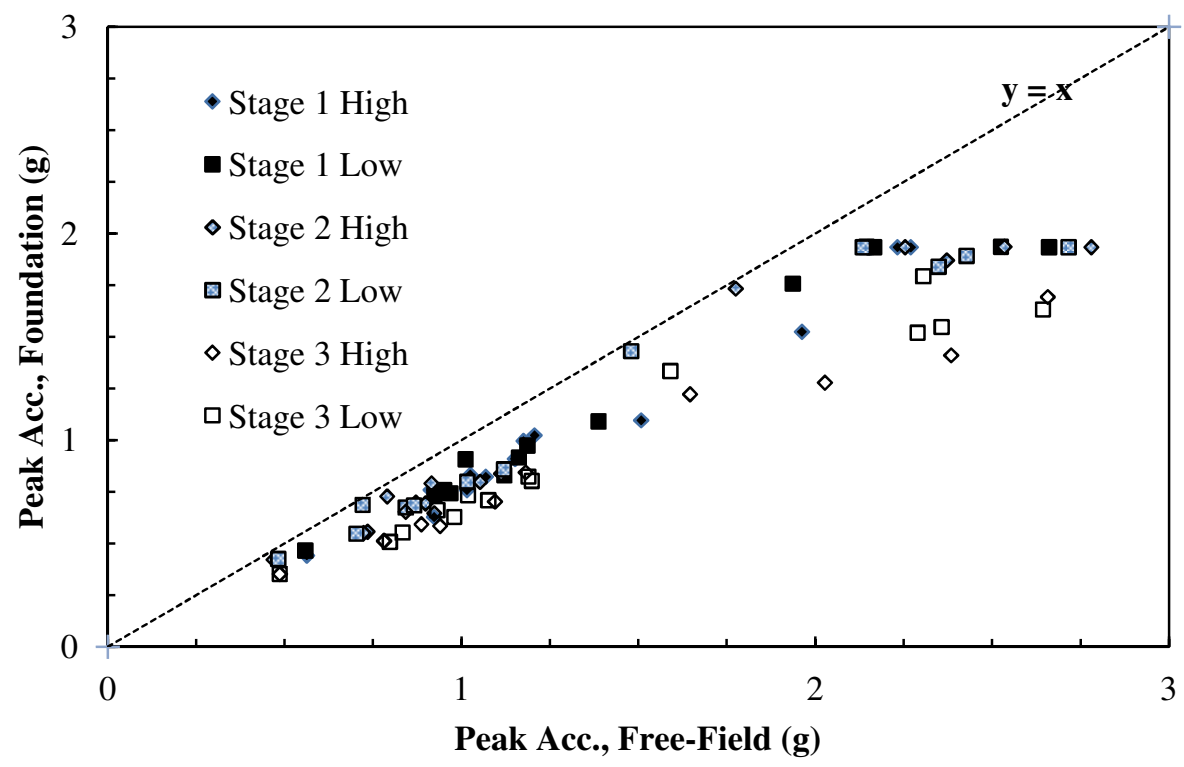

Figure 6-21 Free-field versus foundation level peak acceleration for all the empirical tests

\subsubsection{Data Comparison to Previous Research}

One of the main purposes of this study is to provide empirical results on free-field versus foundation level ground motion recordings to compare to those presented in Stewart (1999b). A compilation of all peak acceleration data is shown in Figure 6-22a. Linear regression trend lines are superimposed for comparison for Stages 1, 2, and 3 data from the study within.

Figure 6-22b compares the linear trend lines from Stewart's results and Stage 1 and 3 of this study. Stage 2 trend line is omitted due to its similarity to Stage 1 results and data ambiguity. The linear trend lines indicate a similar linear relationship between Stewart's surface results to non-embedded Stage 1 data of this study. For surface level comparison, peak accelerations are deamplified at the foundation level relative to free- 
field level during an earthquake. The acceleration deamplification in the foundation level is mostly due to the kinematic interaction effects, as the rigid foundation behaves differently relative to the surrounding softer clay soil during seismic loading.

The results from Stewart-embedded (1998) data and the full-embedment Stage 3 present a similar linear relationship. However, the embedded foundations experienced a stronger motion deamplification. Stage 3 had an embedment ratio $>1$. The deeper embedment resulted in a higher basement wall contact area with the surrounding soil, thus increasing the overall radiation damping that increases deamplification of the ground motion. In addition, the embedded foundation has a deamplified input motion compared to the surface free field motion.

The overall correlation of peak acceleration between the surface level and the embedded foundation level from Stewart's results and the study within is similar, but direct correlation remains difficult to establish as the data have different sample sizes and ranges as shown in Figure 6-22b.

As the amplitude of the peak motion increased, the higher the differences became between the free-field and foundation level peak accelerations; however, the percentage difference between the free-field and foundation level peak acceleration amplitudes remained consistent throughout the testing data. 

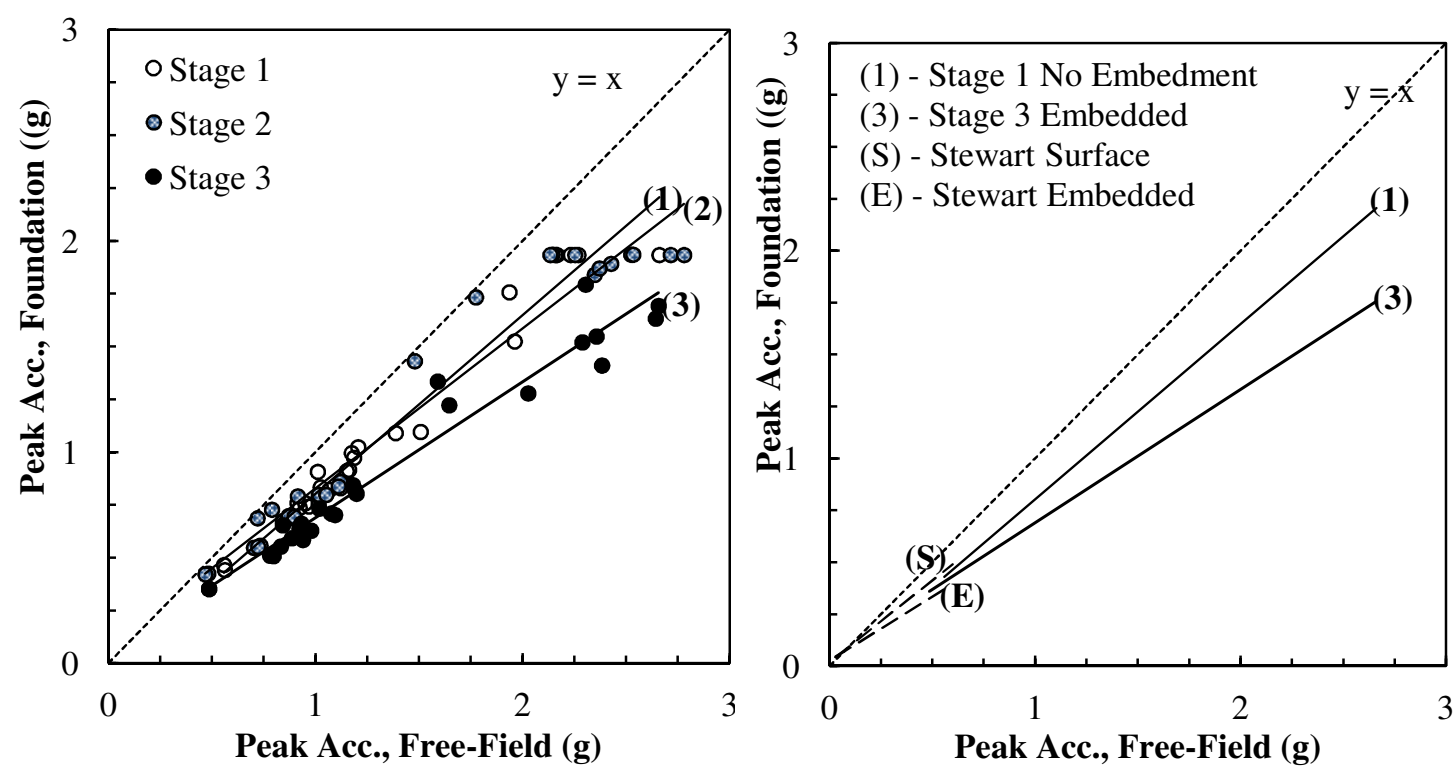

Figure 6-22 a) Linear trend lines for each testing Stage and the original data points b) Comparison of Stewart's results and the study within using linear trendlines (Stewart 1998)

\subsubsection{Effects of Embedment}

The three stages of testing consisted of no embedment, half embedment (at 15 $\mathrm{cm}$ ) of the model foundation, and full embedment (at $30 \mathrm{~cm}$ ). The effects of embedment on peak foundation level acceleration deamplification are represented visually in Figure 6-22a. Results were further quantified by comparing the peak foundation acceleration at each stage for every input motion to observe any motion deamplification.

At Stage 2, where the foundation embedment ratio was 0.58 , the results were similar to those of the non-embedded foundation in Stage 1. By comparing Stage 1 and Stage 2 foundation level peak acceleration, there was an average of $11.4 \%$ deamplification, which was caused by the increased damping of the overall structure due to embedment and different foundation input motion. Furthermore, when comparing the half embedment from Stage 2 to the full embedment of Stage 3, the foundation level 
motion deamplified by an average of $14.8 \%$. Although the embedment increment remained consistent at $15 \mathrm{~cm}$, the overall deamplification from Stage 2 to Stage 3 results was higher than Stage 1 to Stage 2 results by $3.4 \%$.

For the purpose of determining the effects of embedment, results from Stage 1(no embedment) and Stage 3(full embedment with embedment ratio $>1$ ), are plotted in Figure 6-23. Linear trendlines are fitted to each data set. The embedded data show a notable deamplification in the foundation level peak acceleration relative to free-field peak acceleration. The trendlines show that the deamplification effects increase as the model is embedded to as much as $35 \%$ with an average of $30.9 \%$. This observation agrees with Stewart's (1999) finding that foundation embedment increases motion deamplification. The embedded foundation acceleration also deamplified by an average of $27.5 \%$ when compared to the surface acceleration.

The deamplification was caused by a combination of kinematic and inertial interaction effects. Further study is needed to fully understand the degree of influence on motion deamplification from embedment. The notion that free-field and foundation level motions are comparable in amplitude during traditional engineering design needs to be reevaluated as supported by the quantified empirical results. 


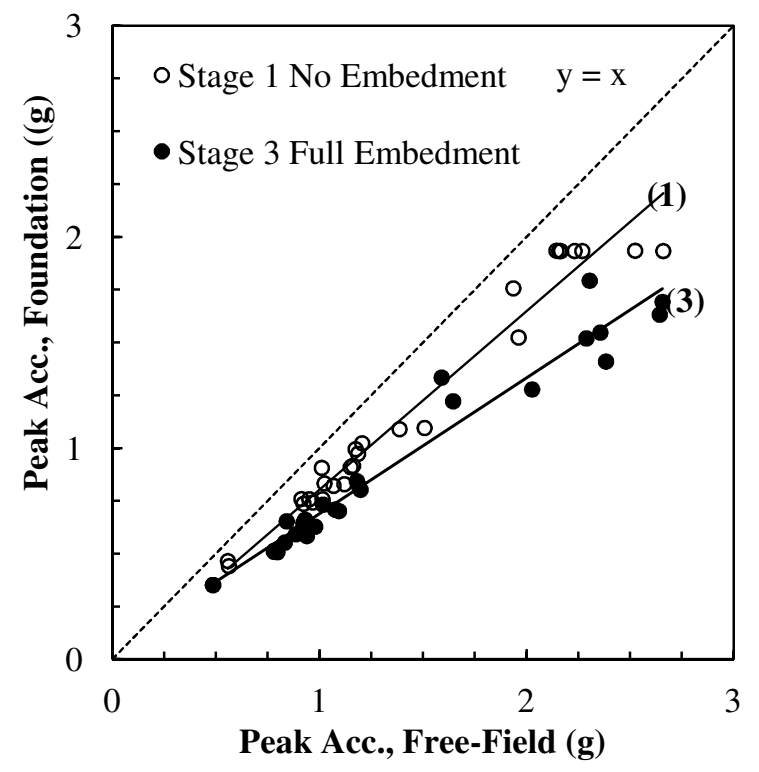

Figure 6-23 Comparison between no-embedment and full-embedment results of peak acceleration

\subsubsection{Effects of Period}

The two model lump mass SDOF setups are Model High and Model low, with a prototype fundamental period of $2.5 \mathrm{~s}$ and $1.5 \mathrm{~s}$, respectively. The purpose is to determine if different setups of the fundamental period would influence the peak foundation acceleration. The result comparison for Model $\mathrm{H}$ and model L is plotted in Figures 6-24, 6-25, and 6-26 for each stage. Linear trendlines are applied to each data set for comparison.

Results from comparing the peak foundation acceleration at each stage between the two model setup do not conclude a consistent correlation. At Stage 1, Model H peak values are on average 6\% higher than Model L. At Stage 2, Model H data are on average $2 \%$ less than Model L. Finally, from Stage 3, Model H results are 7\% higher than Model $\mathrm{L}$ results. The correlations are not consistent with only less than $10 \%$ differences, which could mostly be attributed to natural random variation or data sampling error in empirical 
testing. No significant relationship between the selected high fundamental period, at 1.5 and 2.5 prototype scales, and the peak acceleration values is established from this study.

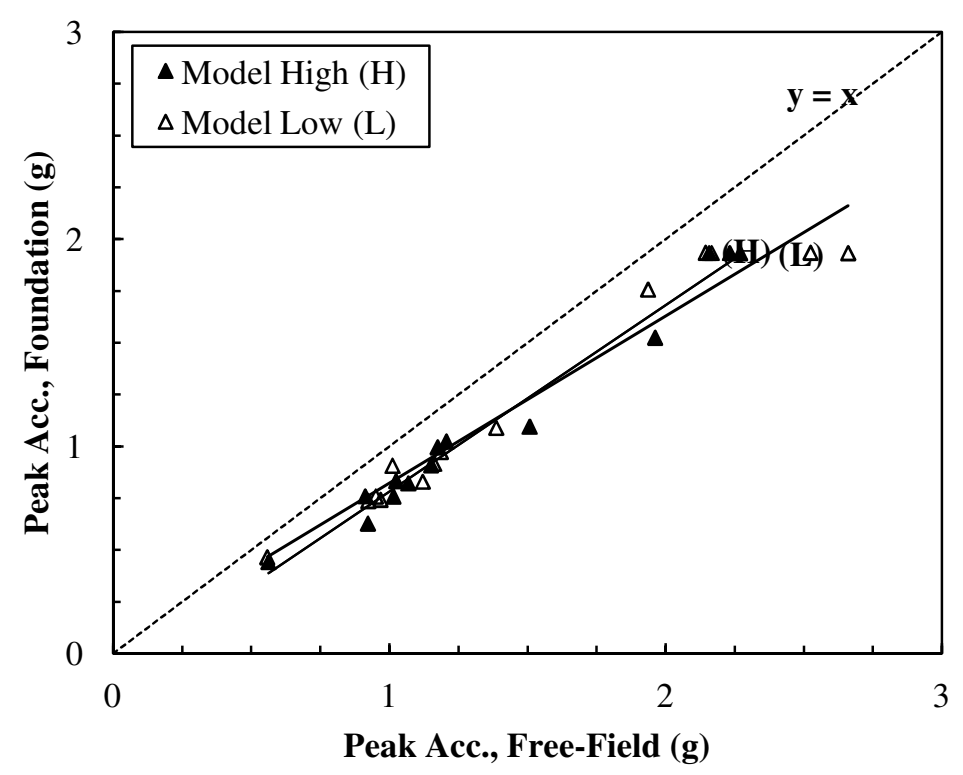

Figure 6-24 Comparison of peak acceleration results between Model High and Model Low for Stage 1

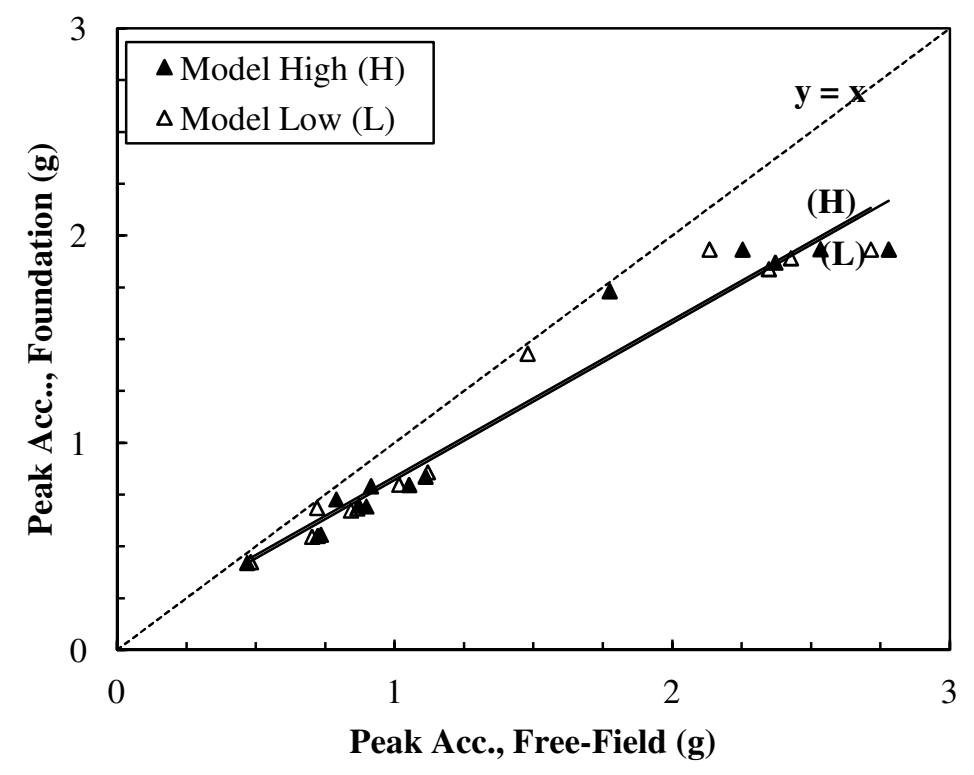

Figure 6-25 Comparison of peak acceleration results between Model High and Model Low for Stage 2 


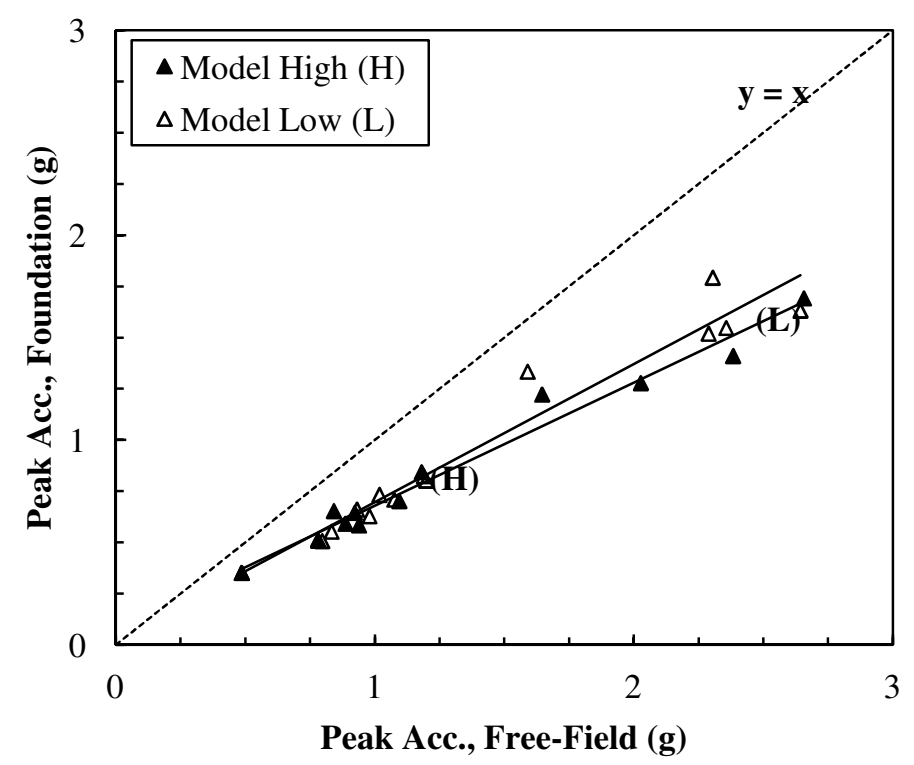

Figure 6-26 Comparison of peak acceleration results between Model High and Model Low for Stage 3

\subsection{Spectral Acceleration Results and Comparison}

\subsubsection{Overview}

Acceleration data from free-field surface and lumped mass accelerometers are generated into 5\% damping spectral values by using MATLAB scripts. The acceleration time step was also adjusted during data transformation to present the spectral results in the prototype scale. The flexible-base period of the foundation $(\square)$ was captured by locating the peak spectral values and the corresponding period using Excel functions. Spectral acceleration at $\square$ results are compared between the free-field and foundation level in Figure 6-27 for each testing Stage.

A general correlation exists between the free-field and the foundation level spectral acceleration at $\square$ as the foundation level spectral accelerations tends to be less than the free-field spectral accelerations. The average percentage reduction in spectral 
acceleration in the foundation level for was 12.6\%, 22.7\%, and 33.4\% for Stage 1, 2 and 3 respectively as shown in Table 6-5.

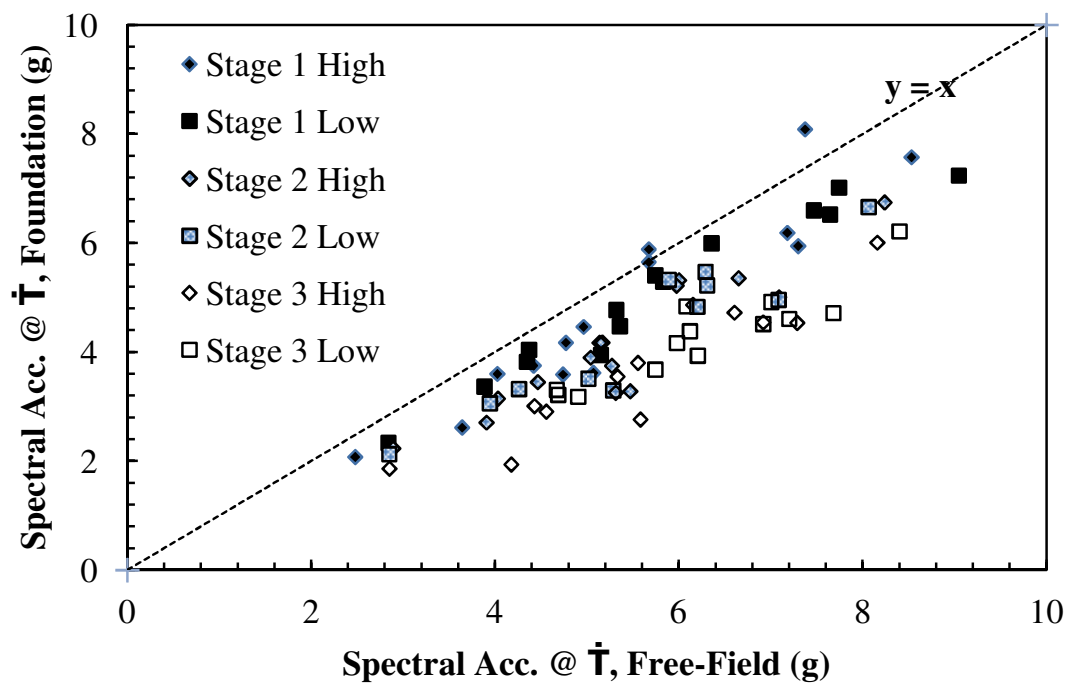

Figure 6-27 Spectral acceleration at the predominant flexible-base period comparison between free-field and foundation level

Table 6-5 Average percent reduction in spectral acceleration at $\square$ of the foundation level when compared to free-field level

\begin{tabular}{rccc} 
Stage\# & Embedment Depth $(\mathrm{cm})$ & $\mathrm{e} / \mathrm{r}$ & Avg. Deamplification (\%) \\
\hline 1 & 0 & 0 & $\mathbf{1 2 . 6}$ \\
2 & 15 & 0.58 & $\mathbf{2 2 . 7}$ \\
3 & 30 & 1.16 & $\mathbf{3 3 . 4}$ \\
\hline
\end{tabular}

\subsubsection{Comparison to Stewart Results}

A compilation of all 5\% damped spectral acceleration data is shown in Figure 628a. Linear regression trend lines are superimposed on Stage 1, 2, and 3 data. Figure 628b compares the linear trend lines generated from Stewart's 5\% damped spectral results to this study.

The linear trend lines indicate a similar linear relationship between Stewart's surface results to the non-embedded Stage 1 data of this study. Likewise, Stewart's 
embedded data and the full-embedment Stage 3 data also present a similar linear relationship. Stewart found an average $20 \%$ reduction, with significant reductions on the order of $40 \%$, for embedded foundation. The average reduction of spectral acceleration at in this study was about $33 \%$ for fully embedded Stage 3 data.

The spectral acceleration at $\square$ best represents the ground motion controlling structural response during seismic loading. The analyzed results from this study agree with Stewart's finding the on effects of embedment on the spectral values deamplification. The ground motion deamplification on the foundation level is directly affected by the depth of embedment. Further analysis is presented in the following section.
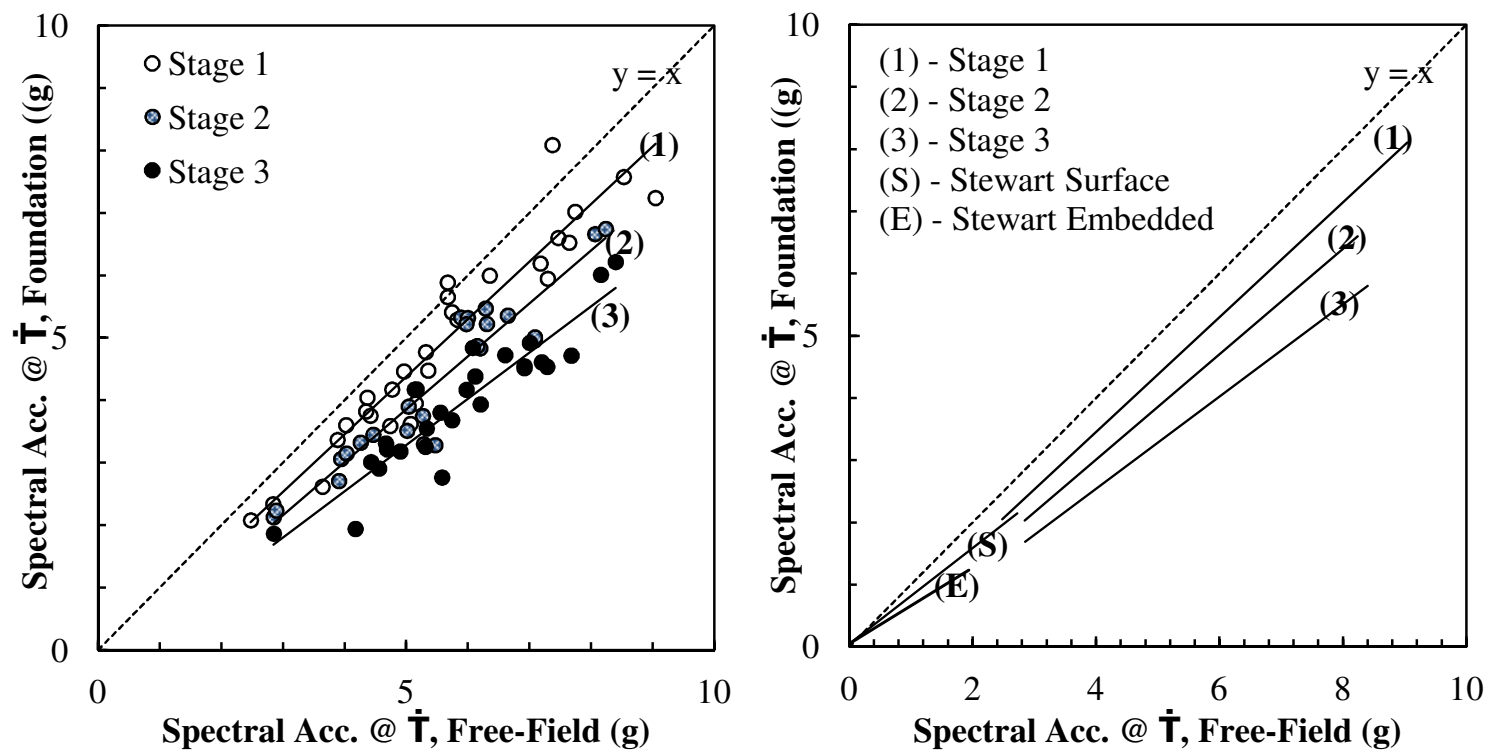

Figure 6-28 a) Comparison of free-field and foundation level 5\% damped spectral acceleration @ ; b) Comparison of Stewart's results and the study within using linear trendlines (Stewart 1998) 


\subsubsection{Embedment Effect}

The effects of embedment on the deamplification of spectral foundation level acceleration at $\square$ are presented as linear trendlines in Figure 6-28. With no embedment at Stage 1, the foundation spectral acceleration was deamplified by an average of $12.6 \%$. When the foundation was embedded for $15 \mathrm{~cm}(\mathrm{e} / \mathrm{r}=0.58)$, the foundation level spectral acceleration deamplification increased to $23.3 \%$. Conversely, there was a $33.4 \%$ reduction in acceleration at Stage 3, which had an embedment ratio of 1.16.

Figure 6-29 shows the positive linear correlation between embedment ratio (e/r) and the average deamplification of spectral acceleration at $\square$ from the study within. The additional foundation embedment depth increased the foundation level spectral acceleration at $\square$. The difference motion amplitudes observed at the foundation level and the free-field level is contributed from multiple reflections and refractions that occurred at the soil layer interface as the seismic waves prorogate through the soil column. The embedded foundation level seismic motion, or the foundation input motion, is considered to be less amplified when compared to the motion observed at the free-field surface. In addition, it is well documented that foundation embedment can increase system damping due to kinematic interaction effects as incidental waves scattering and diffraction from the building foundation during SSI( Bielak 1975; Todorovska 1992; Aviles and PerezRocha 1996). This correlation graph illustrates that kinematic interaction effects on the structure increase as the foundation is embedded deeper into the soil.

The deamplification effects on the foundation input motion are also caused by the increase of hysteretic damping during seismic excitation due to the additional lateral foundation basement wall-to-soil contact area (Aviles and Perez-Rocha 1996). As the 
foundation is embedded deeper into the soil, the physical interaction between the basement walls and the surrounding cohesive soil can reduce the seismic response in the structure, thus leading to reduced amplitude at the embedded foundation level. The higher embedment ratio results in a more pronounced system motion deamplification as the wall-to-soil contact area increase, which produces higher hysteretic damping; therefore, the foundation input motion is noticeably lower than the free-field motion, where no superstructure is altering the waveform. For the surface level foundation in this study, there is a significant motion deamplification relative to free-field motion. However, only a slight reduction in surface foundation level spectral acceleration was observed according to Stewart (1999b)'s study. Since the testing setup of this experiment was confined to soft soil condition with a rigid scale steel foundation only, the structure-tosoil-stiffness ratio $(1 / \sigma)$ remains constant and is assumed to be reasonably high. Hence, the results from this study cannot be viewed as directly parallel to actual prototype observation results, such as those collected by Stewart.

The correlation shown in Figure 6-29 only represents results for the empirical testing setup used in this study, which had a high structure to soil stiffness ratio, and is not applicable for other site conditions without further examination. By using a controlled laboratory, scale model setup, and with limited factors, the results served to investigate an unexplored condition. The relationship between the embedment ratio and motion deamplification isn't application to other conditions. In addition, the structure-to-soil stiffness, which can affect the influence of soil-structure-interaction considerably as suggested by Stewart at el. (1998), remains significant and constant in this study. Although the comparison is presented as a function of embedment ratio, the equivalent 
radius of the foundation stayed the same through the test. The linear relationship, as suggested here, needs addition empirical and analytical studies with a larger testing sample group by isolating the different degrees of influence on factors, such as foundation structure stiffness, soil style, soil uniformity, and foundation size and shape to be valid. Further investigation in this matter is necessary before establishing any credible correlation or trend on this regard.

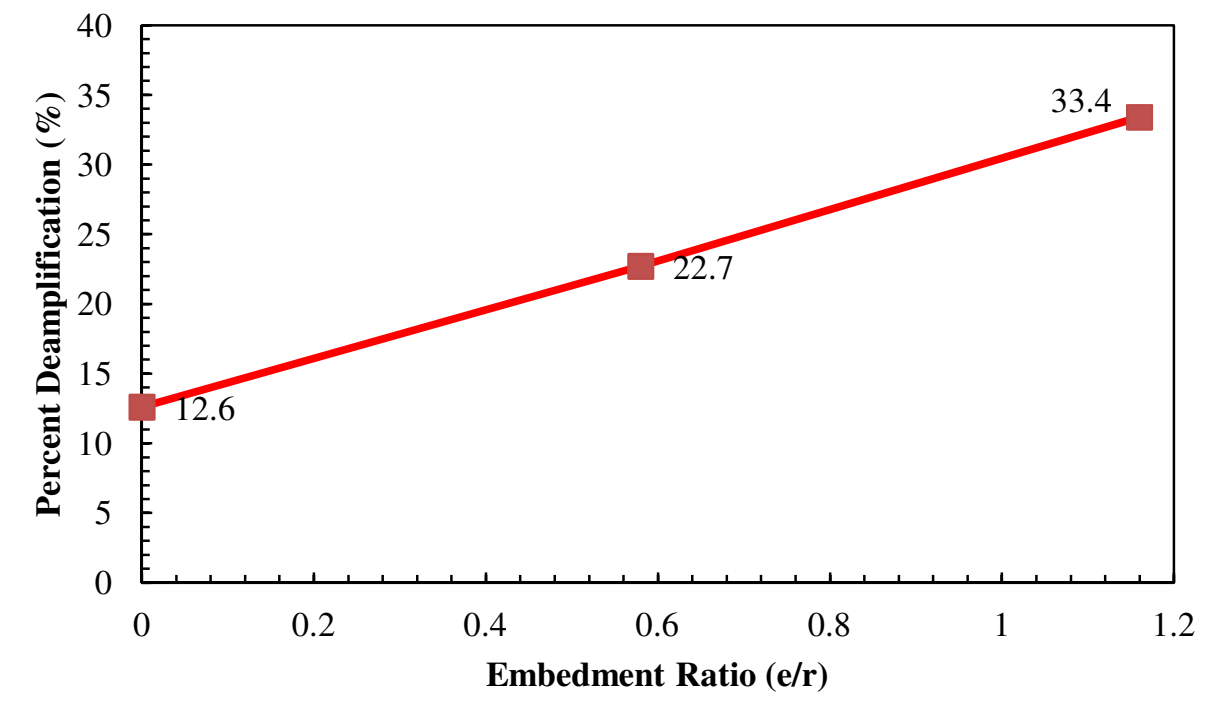

Figure 6-29 Correlation between embedment ratio (e/r) and the average deamplification of spectral acceleration at

\subsubsection{Period Change Effect}

Spectral acceleration at $\square$ comparison between free-field and foundation level for Model $\mathrm{H}$ and model L are plotted in Figures 6-30, 6-31, and 6-32 for each stage. Linear trendlines are applied to each data set for comparison. Similar to the results from peak acceleration analysis, comparisons of spectral acceleration at $\square$ at each stage between the two model setup did not have pronouncing correlations. At Stage 1, Model H peak values were, on average, 6\% higher than Model L. At Stage 2, Model H data were on average 
2\% less than Model L. Finally, at Stage 3, Model H results were 10\% higher than Model $\mathrm{L}$ results. The prototype fundamental period was much higher than the motion spectral period range, usually at much lower period $(\mathrm{T}<1)$; therefore, no significant deamplification between the spectral acceleration at free-field and the model at the selected high fundamental periods at 1.5 and 2.5 prototype scale was established from this study.

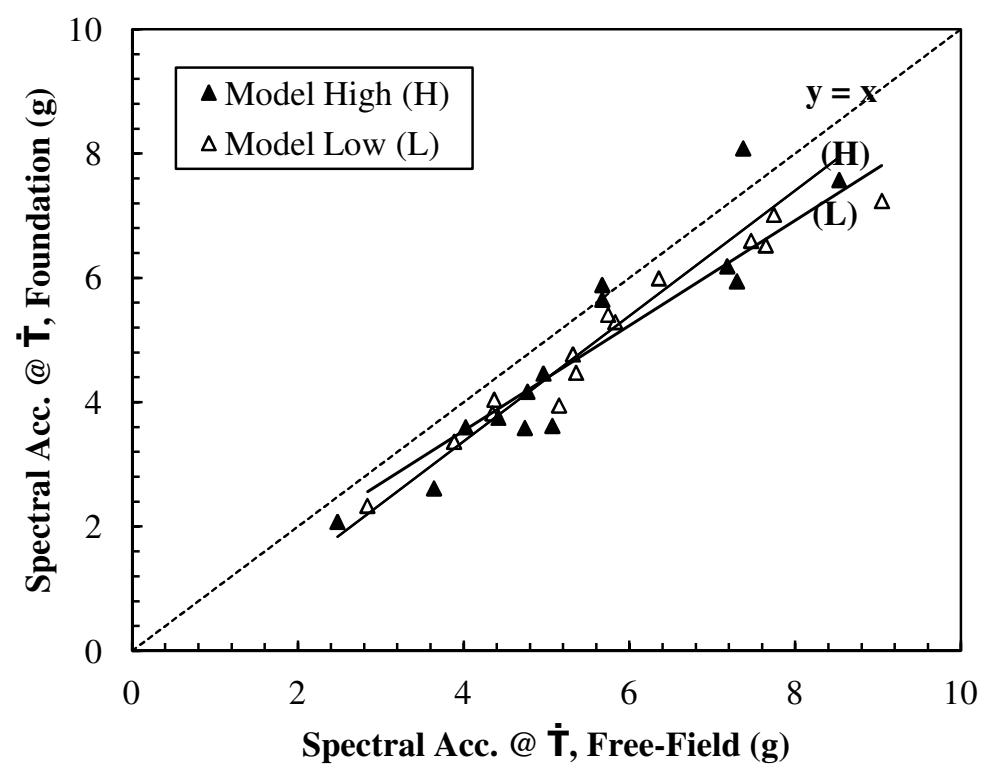

Figure 6-30 Comparison of spectral acceleration at $\square$ between Model High and Model Low for Stage 1 


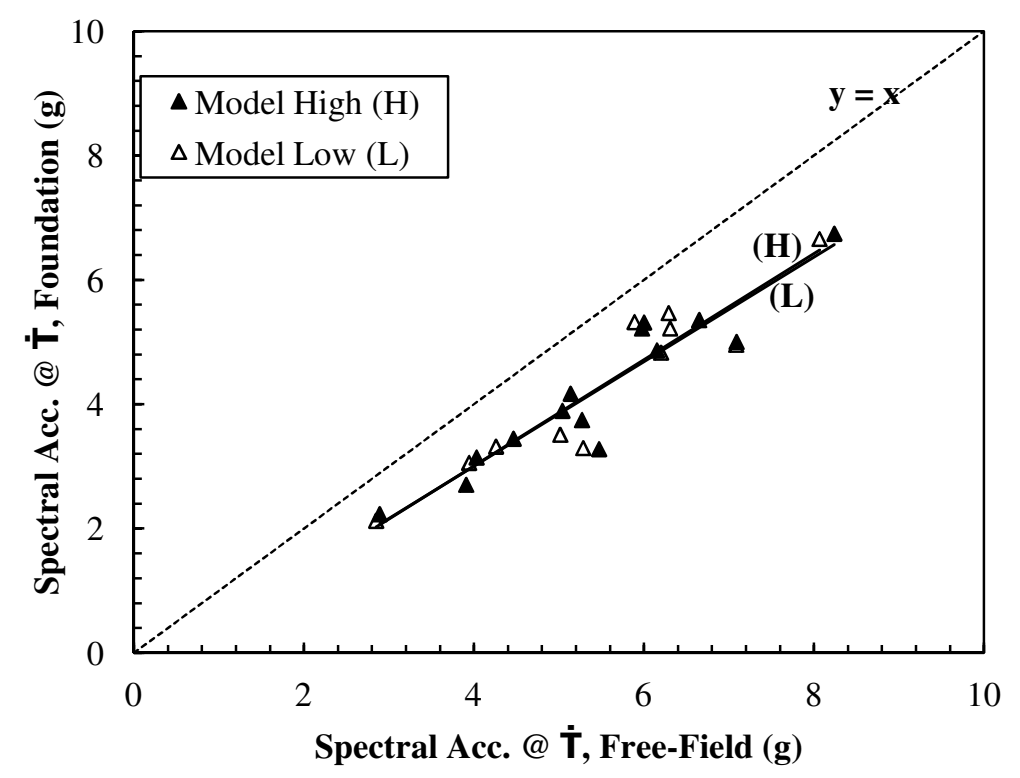

Figure 6-31 Comparison of spectral acceleration at $\square$ between Model High and Model Low for Stage 2

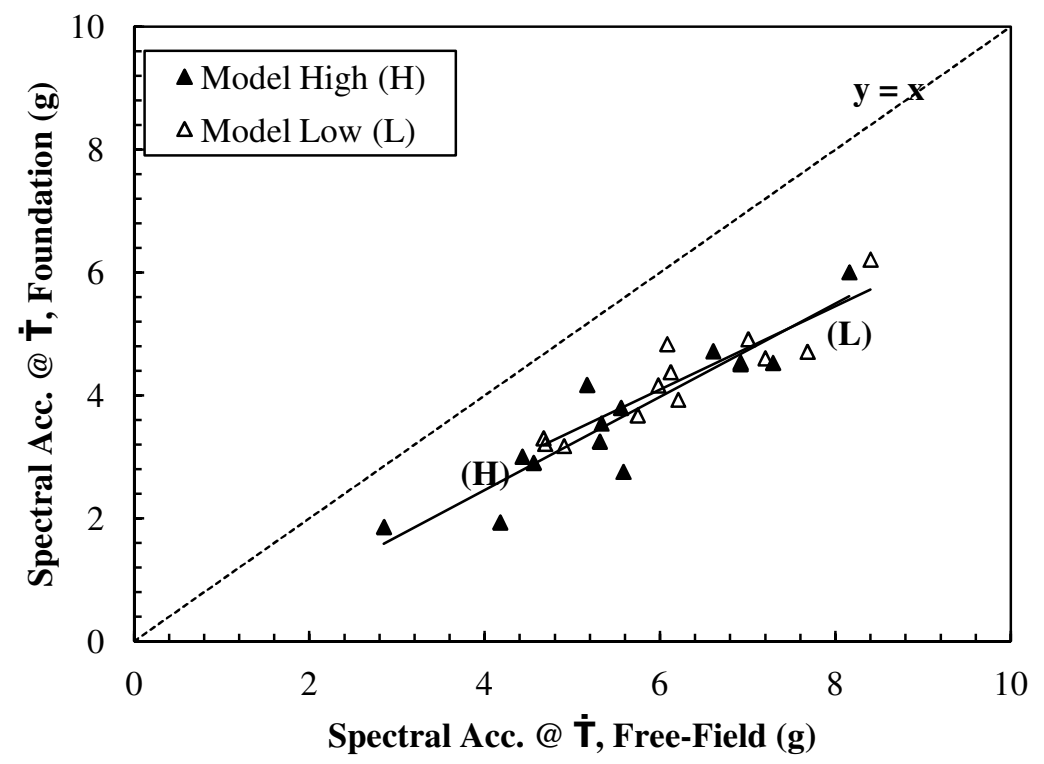

Figure 6-32 Comparison of spectral acceleration at $\square$ between Model High and Model Low for Stage 3 


\subsubsection{Period Lengthening}

Stewart justifies inertial SSI effects in terms of period lengthening ratio and foundation damping factors that are based on simple analytical equations and assumptions. In the study within, the empirical data obtained were used to determine the period lengthening ratio. Similar to other experimental testing programs, erroneous results can occur due to human error, equipment failure, and natural random data variation. Data processing is necessary to eliminate such errors as frequently as possible. From the experimental data, the SDOF lumped mass fundamental period were confirmed, and ambiguous data were omitted.

Using the lumped mass fundamental period data at Stage 1 and Stage 3, the nonembedded foundation results were compared to the fully embedded foundation results to compute the period lengthening ratio ( $\square 3 / \square 1$ ). The average period lengthening ratio for Model High and Low were, 0.99 and 0.93, respectively. Results are presented in Table 6 6. Results from motions L09090, IPV045, and IPV135 were ignored because multiple mode responses were observed in the spectral results, and the $1^{\text {st }}$ mode period was difficult to establish.

There was no indication of any significant period lengthening effects in the SDOF lumped mass in this study. Stewart (1999b) states that period lengthening for long-period $(T>2 \mathrm{~s})$ structures is negligible and can be neglected. Inertia interaction effects are usually more pronounced in lower period structures. The selected model setups in this study had fundamental periods that were too high for the seismic loading to induce any significant effect. However, kinematic interaction effects can increase the foundation damping and decrease the system period from foundation embedment (Aviles and Perez- 
Rocha 1996). For the particular experimental setup within, kinematic interaction effects had a greater influence on the overall system than inertial interaction effects as the system periods experienced a slight decrease when comparing the full embedment to nonembedment.

Table 6-6 Average period lengthening ratio from this study

\begin{tabular}{clcc}
$\#$ & Motion & Model (H) $\square 3 / \square 1$ & Model (L) $\square 3 / \square 1$ \\
\hline 1 & ELC180 & 0.99 & 0.88 \\
2 & ELC270 & 1.04 & 0.89 \\
3 & JOS000 & 0.99 & 0.91 \\
4 & JOS090 & 0.99 & 0.92 \\
5 & TCU_N & 0.92 & 0.91 \\
6 & TCU_W & 0.98 & 0.88 \\
7 & CPM000 & 0.98 & 1.00 \\
8 & CPM090 & 1.00 & $\mathrm{n} / \mathrm{a}$ \\
9 & LGP000 & 0.98 & 0.95 \\
10 & LGP090 & n/a & 0.98 \\
11 & L09000 & 1.07 & 0.98 \\
\hline \hline & Average & $\mathbf{0 . 9 9}$ & $\mathbf{0 . 9 3}$
\end{tabular}

\subsection{Summary}

Phase I testing wais used to confirm the SDOF first mode period at the current lumped mass configuration. A $20 \mathrm{lb}$. weight was chosen and fixed at $52 \mathrm{~cm}$ and $72 \mathrm{~cm}$ appropriate lumped mass SDOF setup on the foundation to achieve prototype periods of 1.5 and $2.5 \mathrm{~s}$ of tall buildings, respectively.

Phase II testing compares the model soil column results and accelerometer performance with Crosariol (2010) results. In addition, scale model behavior during seismic loading was studied. Highlights of the results and analysis are listed below:

- From the T-bar results, the average soil strength increased with depth. 
- By comparing different T-bar results taken at one-month interval, the average soil strength gain for the first month was $13 \%$, and $8 \%$ for the second month.

- From the hammer blow test, the average shear wave velocity for layer 3 to 2 and 2 to 1 were 35.1 and $42.3 \mathrm{~m} / \mathrm{s}$ respectively, and the overall average shear wave velocity for the lower soil column was $38.7 \mathrm{~m} / \mathrm{s}$.

- From the spectral response comparison, the predominant resonate period of the model soil column decreased $31 \%$ from an average of $0.51 \mathrm{~s}$ to an average of $0.35 \mathrm{~s}$ from Crosariol's results. A stiffer soil column had a lower predominant period.

- From the displacement wire pot data, the relative displacement between the table and the soil surface was minimal, as the soil column adhered to the shake table movement closely during shake table testing.

- Foundation horizontal accelerometers data were analyzed, and no foundation twisting behavior was observed. In addition, foundation vertical accelerometer data showed no noticible foundation rocking effects during shake table testing. Summary of peak acceleration and spectral acceleration at $\square$ data are presented in Table 6-7 and 6-8. Some of the observations from comparing the empirical results of this study are summarized as follow:

- The correlation between free-field and foundation level peak accelerations within this study agree with the trends observed in Stewart (1999b). Embedded foundations experienced higher motion deamplifcation than non-embeded foundations. Similarly, the spectral acceleration at $\square$ comparison shows a similar result. 
- Embedment increases the overall motion deamplifcation on the foundation level relative to the free-field level. The degree of deamplifcaition in motion increases with deeper foundation embedment.

- For spectral acceleration at $\square$ embedment effect, there is a strong correlation between the embedment ratio and motion deamplification percentage. The higher the embedment ratio, the higher the motion deamplification of spectral acceleration on the foundation. This behavior is caused by the additional soil-tofoundation wall contact surface that results in a greater overall system damping that lowers the motion amplitude.

- There was little to no effect on the motion amplitutde between the two lumpedmass setup chosen for this study. That includes both peak acceleration data and spectral acceleration at $\square$.

- No pronounced period lengthening effect was found from the empirical results. The selected prototype model with high periods $(\mathrm{T}=1.5 \mathrm{~s}$ and $2.5 \mathrm{~s})$ did not experience any inertial interaction effects from the period lengthening effect due to foundation embedment.

- As foundation is embedded, kinematic interactione effects can increase the system damping and decrease the system period. Both behaviors were observed from the data collected.

- A bigger data sample pool is needed to establish reliable correlations. 
Table 6-7 Summary of peak acceleration data

\begin{tabular}{|c|c|c|c|c|c|c|c|c|c|c|c|c|}
\hline \multirow[b]{3}{*}{ Motion } & \multicolumn{4}{|c|}{ Stage 1} & \multicolumn{4}{|c|}{ Stage 2} & \multicolumn{4}{|c|}{ Stage 3} \\
\hline & \multicolumn{2}{|c|}{ Model High } & \multicolumn{2}{|c|}{ Model Low } & \multicolumn{2}{|c|}{ Model High } & \multicolumn{2}{|c|}{ Model Low } & \multicolumn{2}{|c|}{ Model High } & \multicolumn{2}{|c|}{ Model Low } \\
\hline & $5 \mathrm{~F}(\mathrm{~g})$ & Model $(\mathrm{g})$ & $5 \mathrm{~F}(\mathrm{~g})$ & Model (g) & $5 \mathrm{~F}(\mathrm{~g})$ & Model (g) & $5 \mathrm{~F}(\mathrm{~g})$ & Model (g) & $5 \mathrm{~F}(\mathrm{~g})$ & Model (g) & $5 \mathrm{~F}(\mathrm{~g})$ & Model (g) \\
\hline ELCEN180 & 1.15 & 0.91 & 1.16 & 0.92 & 1.05 & 0.80 & 1.02 & 0.80 & 1.09 & 0.70 & 1.20 & 0.80 \\
\hline ELCEN270 & 0.91 & 0.76 & 0.95 & 0.76 & 0.73 & 0.56 & $\mathrm{n} / \mathrm{a}$ & $\mathrm{n} / \mathrm{a}$ & 0.78 & 0.51 & 0.83 & 0.55 \\
\hline JOSH000 & 1.02 & 0.83 & 1.19 & 0.97 & 0.91 & 0.79 & $\mathrm{n} / \mathrm{a}$ & $\mathrm{n} / \mathrm{a}$ & 0.92 & 0.65 & 1.02 & 0.73 \\
\hline JOSH090 & 1.07 & 0.82 & 1.12 & 0.83 & 0.87 & 0.70 & 0.84 & 0.67 & 0.89 & 0.59 & 1.08 & 0.71 \\
\hline CHICHI-N & 0.92 & 0.63 & 0.97 & 0.74 & 0.72 & 0.55 & 0.70 & 0.55 & 0.78 & 0.51 & 0.80 & 0.51 \\
\hline CHICHI-W & 1.17 & 1.00 & 1.39 & 1.09 & 1.11 & 0.84 & 1.12 & 0.86 & 1.18 & 0.84 & 1.19 & 0.82 \\
\hline СРМ000 & 2.23 & 1.93 & 2.66 & 1.93 & 2.78 & 1.93 & 2.72 & 1.93 & 2.66 & 1.69 & 2.64 & 1.63 \\
\hline СРМ090 & 2.27 & 1.93 & 2.17 & 1.93 & 2.25 & 1.93 & 2.13 & 1.93 & $\mathrm{n} / \mathrm{a}$ & $\mathrm{n} / \mathrm{a}$ & 2.29 & 1.52 \\
\hline HSUP045 & 0.56 & 0.44 & 0.56 & 0.47 & 0.47 & 0.42 & 0.48 & 0.43 & 0.49 & 0.35 & 0.49 & 0.35 \\
\hline HSUP135 & 1.21 & 1.02 & 1.01 & 0.91 & 0.79 & 0.73 & 0.72 & 0.69 & 0.84 & 0.65 & 0.93 & 0.66 \\
\hline L09000 & 1.01 & 0.76 & 0.92 & 0.74 & 0.90 & 0.69 & 0.87 & 0.68 & 0.94 & 0.58 & 0.98 & 0.63 \\
\hline L09090 & 2.16 & 1.93 & 1.94 & 1.76 & 1.77 & 1.73 & 1.48 & 1.43 & 1.65 & 1.22 & 1.59 & 1.33 \\
\hline LGP000 & 1.96 & 1.52 & 2.52 & 1.94 & 2.53 & 1.94 & 2.43 & 1.89 & 2.03 & 1.28 & 2.30 & 1.79 \\
\hline LGP090 & 1.51 & 1.10 & 2.14 & 1.94 & 2.37 & 1.87 & 2.35 & 1.84 & 2.38 & 1.41 & 2.36 & 1.55 \\
\hline
\end{tabular}

Table 6-8 Summery of spectral acceleration at $\square$ data

\begin{tabular}{|c|c|c|c|c|c|c|c|c|c|c|c|c|}
\hline \multirow[b]{3}{*}{ Motion } & \multicolumn{4}{|c|}{ Stage 1} & \multicolumn{4}{|c|}{ Stage 2} & \multicolumn{4}{|c|}{ Stage 3} \\
\hline & \multicolumn{2}{|c|}{ Model High } & \multicolumn{2}{|c|}{ Model Low } & \multicolumn{2}{|c|}{ Model High } & \multicolumn{2}{|c|}{ Model Low } & \multicolumn{2}{|c|}{ Model High } & \multicolumn{2}{|c|}{ Model Low } \\
\hline & $5 \mathrm{~F}(\mathrm{~g})$ & Model (g) & $5 \mathrm{~F}(\mathrm{~g})$ & $\operatorname{Model}(\mathrm{g})$ & $5 \mathrm{~F}(\mathrm{~g})$ & Model (g) & $5 \mathrm{~F}(\mathrm{~g})$ & Model (g) & $5 \mathrm{~F}(\mathrm{~g})$ & Model (g) & $5 \mathrm{~F}(\mathrm{~g})$ & Model (g) \\
\hline ELCEN180 & 5.07 & 3.62 & 5.36 & 4.48 & 5.47 & 3.28 & 5.28 & 3.30 & 5.58 & 2.76 & 6.21 & 3.93 \\
\hline ELCEN270 & 3.64 & 2.62 & 3.89 & 3.36 & 3.91 & 2.71 & $\mathrm{n} / \mathrm{a}$ & $\mathrm{n} / \mathrm{a}$ & 4.18 & 2.94 & 4.67 & 3.31 \\
\hline JOSH000 & 4.96 & 4.46 & 5.74 & 5.41 & 5.14 & 4.17 & $\mathrm{n} / \mathrm{a}$ & $\mathrm{n} / \mathrm{a}$ & 5.55 & 3.80 & 6.12 & 4.38 \\
\hline JOSH090 & 4.77 & 4.17 & 5.32 & 4.77 & 5.04 & 3.90 & $\mathrm{n} / \mathrm{a}$ & $\mathrm{n} / \mathrm{a}$ & 5.33 & 3.55 & 5.98 & 4.17 \\
\hline CHICHI-N & 4.02 & 3.60 & 4.37 & 4.04 & 4.03 & 3.15 & 3.94 & 3.06 & 4.43 & 3.01 & 4.69 & 3.22 \\
\hline CHICHI-W & 7.30 & 5.95 & 7.64 & 6.52 & 7.09 & 5.01 & 7.08 & 4.96 & 7.29 & 4.53 & 7.20 & 4.61 \\
\hline СРМ000 & 7.37 & 8.09 & 7.47 & 6.60 & 6.00 & 5.32 & 5.89 & 5.32 & 6.61 & 4.72 & 7.00 & 4.92 \\
\hline СРM090 & 7.18 & 6.19 & 5.83 & 5.29 & 6.15 & 4.87 & 6.20 & 4.83 & $\mathrm{n} / \mathrm{a}$ & $\mathrm{n} / \mathrm{a}$ & 6.92 & 4.51 \\
\hline HSUP045 & 8.53 & 7.57 & 7.74 & 7.02 & 5.98 & 5.22 & 6.29 & 5.47 & 5.17 & 4.17 & 6.08 & 4.84 \\
\hline HSUP135 & 5.67 & 5.65 & 9.05 & 7.24 & 8.24 & 6.74 & 8.06 & 6.66 & 8.16 & 6.01 & 8.40 & 6.21 \\
\hline L09000 & 4.42 & 3.75 & 4.35 & 3.82 & 4.47 & 3.45 & 4.26 & 3.32 & 4.56 & 2.91 & 4.90 & 3.18 \\
\hline L09090 & 5.67 & 5.89 & 6.35 & 5.99 & 6.65 & 5.36 & 6.31 & 5.22 & 6.92 & 4.54 & 7.68 & 4.71 \\
\hline LGP000 & 2.48 & 2.08 & 2.84 & 2.34 & 2.89 & 2.23 & 2.85 & 2.13 & 2.85 & 1.86 & $\mathrm{n} / \mathrm{a}$ & $\mathrm{n} / \mathrm{a}$ \\
\hline LGP090 & 4.74 & 3.59 & 5.15 & 3.95 & 5.27 & 3.75 & 5.01 & 3.51 & 5.31 & 3.25 & 5.74 & 3.68 \\
\hline
\end{tabular}




\section{CHAPTER 7 - SUMMARY AND CONCLUSIONS}

\subsection{Scope of Research}

Seismic soil-structure-interaction (SSI) is known to influence building dynamic behavior. Stewart et al. (1998) compares data of the free-field motion and the foundation level motion, which is often deamplified due to kinematic and inertial interaction effects. Stewart's results suggest peak ground accelerations and spectral accelerations deamplification at the foundation-level when compared to the free-field data. Overall, empirical data regarding SSI effects is limited for shallow foundations with embedment, especially in soft cohesive soils. More data is needed to establish the effects of SSI due to foundation embedment.

Using similitude scaling relations, a scale model was developed with prototype fundamental periods of $1.5 \mathrm{~s}$ and $2.5 \mathrm{~s}$ Shake table tests were conducted at three stages with foundation embedment ratios $(\mathrm{e} / \mathrm{r})$ of $0,0.58$, and 1.16 . Empirical lab testing data from a controlled environment were collected and compared to Stewart's results. The testing platform to explore SSI effects in soft cohesive soil in a 1-g testing environment was first developed by Crosariol (2010) at Cal Poly. From the experimental shake table testing, accelerations from the scale model and soil column were measured.

This chapter summarizes the notable research findings from empirical shake table testing and data comparison to Stewart's results. Recommendations for improvement of the testing platform and potential future research topics are also included in the following sections. 


\subsection{Research Findings}

Model soil testing results suggested that the recycled and reconstituted soil from previous experiments has a slightly higher soil strength due to a lower average water content. The natural period of the soil column decreased from an average of $0.51 \mathrm{sec}$ to $0.35 \mathrm{sec}$ due to a stiffer soil colum. The results indicate that the model soil allows for satisfactory test repeatability and can be reused as long as the soil is checked and monitored for soil properties.

The shake table and soil column moved in unison during seismic excitation as evidenced by the displacement wire pot data, which measured the shake table level and soil surface level displacements. The displacement results were reasonably similar and warranted the testing platform to provide valid free-field responses imperative for this study.

Althought no data were recorded for soil column twisting and rocking behavior, twisting and rocking were not observed in the scale model foundation as suggested by the acceleration data, which were collected from two horizontal and two vertical accelerometers on the foundation. This limited the potential of foundation twisting or rocking behaviors that influence the foundation level motion amplitute.

Empirical results from this study were compared to results from Stewart et al. (1999b). The correlation between free-field and foundation level peak accelerations within this study agree with the trends as observed in Stewart et al. (1999b). Embedded foundations experienced higher motion deamplifcation than non-embeded foundations. Similarly, the spectral acceleration at $\square$ comparison showed reasonable motion deamplification. Despite having higher acceleration amplitude, the empirical data 
obtained from this study provide reasonable outcomes, similar to the correlations established by Stewart et al. (1999b).

The degree of motion deamplifcaition at the foundation level increased with deeper embedment depth. Likewise, spectral acceleration at $\square$, there was a strong linear correlation between the motion deamplification and foundation embedment ratio. High embedment ratio resulted in stronger deamplification of spectral acceleration on the foundation due to spatial variation of foundation input motion as opposed to the free-field motion and the additional damping associated with the increase of soil to foundation wall contact area.

It is clear from the data that period lengthening effects were not pronounced in this study. The inertial interaction effects, which are more common in lower periods sturctures, were not a dominating factor during SSI for longer period structures. The lack of a short period building scale model in this study limited the direct analysis on how short and long period buildings behave differently as a result of period lengthening effects. A slight period decrease from foundation embedment is observed. Such behavior can be contributed from kinematic interaction effects.

Another inertial interaction effect concern, the foundation damping factor, cannot be quantified in this study. Therefore, no actual values were determined to conclude if foundation damping increased. Based on prior study (Stewart et al. 1998), the deeper the embedment depth, the more exterior foundation walls come in contact with the surrounding soft cohesive soil, and radiation damping is inevitably increased. Foundation radiation damping during seismic loading is another important issue, along with kinematic and inertial interaction effects in SSI, but is beyond the scope of this study. 


\subsection{Recommendations for Improvement and Future Research}

Outlined below are lessons learned from this study that can be applied for future research, as well as recommendations for future studies using Cal Poly's shake table testing platform:

- Increase the moment of inertia on the SDOF rod in place of the rail guides to ensure one directional oscillation.

- Select a lower fundamental period of a prototype model to study the period lengthening effects from seismic motions.

- Include embedment ratio (e/r) less than 0.5 to further study the effects of shallow embedment.

- Measure the foundation displacement during seismic loading to monitor foundation drift and establish a load-displacement curve.

- Investigate the effects of foundation shape on SSI by using different foundation dimensions.

- Investigate SSI effects on individual footing, such as individual spread footing.

- Investigate radiation damping effects as a result of embedment.

- Use pressure transducers on the embedded exterior wall to measure dynamic earth pressure load.

- Investigate SSI effects on other types of structures such as retaining walls, embankment, footings, and deep foundations.

- Use a modeling of models approach to exam the different scale relations validity.

- Experiment with varying soil type layering. Perhaps use a real life site soil profile as the prototype for the soil column. 


\section{REFERENCES}

Applied Technology Council (1978). "Tentative provisions for the development of seismic regulations for buildings: a cooperative effort with the design profession, building code interests, and the research community," Rpt. No. ATC 3-06, U.S. Dept. of Commerce, National Bur. Of Standards, Washington DC.

Apsel, R.J. and Luco, J.E. (1987). "Impedance functions for foundations embedded in a layered medium: an integral equation approach," J. Earthquake Engrg. Struct. Dynamics, 15(2), 213-231.

Aubry, D, and Clouteau,D.(1992)" Asubdomain approach to dynamic Soil-structure interaction. In:Recent advances in earthquake engineering and structural dynamics." Ouest Editions/AFPS, Nantes, France, pp 251-272

Aviles, J. and Perez-Rocha, L.E. (1996). "Evaluation of interaction effects on the system period and the system damping due to foundation embedment and layer depth," Soil Dynamics and Earthquake Engrg., 15(1), 11-27.

Avilés, J., \& Pérez-Rocha, L. E. (1998). "Effects of foundation embedment during building-soil interaction." Earthquake Engineering \& Structural Dynamics, 27(12), $1523-1540$.

Bazzurro, P., and C. A. Cornell (2004). "Ground-motion amplification in nonlinear soil sites with uncertain properties," Bull. Seism. Soc. no. 6, 2090-2109

Bielak, J. (1975). "Dynamic behavior of structures with embedded foundations," $J$. Earthquake Engrg. Struct. Dynamics, 3(3), 259-274.

Borja, R.I., Chao, H.Y., Montáns, F.J., and Lin, C.H.(1999) "SSI effects on ground motion at Lotung LSST site," Journal of Geotechnical and Geoenvironmental Engineering, ASCE, Vol. 125, No. 9, pp. 760-770.

Buehler, M., Wienbroer, H., \& Rebstock, D. (2006). "A full seismic soil-foundationstructure interaction approach." 1st European Conference on Earthquake Engineering and Engineering Seismology Geneva.

Building Seismic Safety Council, BSSC (1997). "NEHRP Recommended provisions for seismic regulations for new buildings, Part 1, Provisions and Part 2, Commentary" Rpt. No. FEMA 302 \& 303, Federal Emergency Management Agency, Washington D.C.

Chopra, A.K. (2001). Dynamics of Structures: Theory and Applications to Earthquake Engineering. 2nd Edition, New Jersey: Prentice Hall.

Clouteau D (2005) MISS 6.4: manuel utilisateur: version 2.3.. Chatenay-Malabry, France 
Clouteau, D. and Aubry, D. (2003) "Computational soil-structure interaction. In:

Boundary element methods for soil-structure interaction," Kluwer Academic Publishers, chap. 2, pp 61-126

Crosariol, V. (2010) "Scaled 1g Testing of Soil-Structure-Interaction Models" Masters Thesis, Cal Poly San Luis Obispo.

Day, S.M. (1977). "Finite element analysis of seismic scattering problems," Ph.D.

Dissertation, Univ. of California, San Diego.

Dickenson, S. E. (1994). Dynamic response of soft and deep cohesive soils during the Loma Prieta Earthquake of October 17, 1989. University of California, Berkeley. Gruber, A. (1996). "Stress-Strain Testing of a Model Soil for Earthquake Simulation." Rpt. to the Dept. of Civil Eng., University of California, Berkeley.

Elsabee, F. and Morray, J.P. (1977). "Dynamic behavior of embedded foundations," Rpt. No. R77-33, Dept. of Civil Engrg., MIT, Cambridge, Mass.

Gajan, S., Hutchinson, T. C., Kutter, B. L., Raychowdhury, P., Ugalde, J. A., and Stewart, J. P. (2008) "Numerical models for the analysis and performance-based design of shallow foundations subjected to seismic loading ", PEER Report No. 2007/04, Pacific Earthquake Engineering Research Center (PEER), University of California, Berkeley, pp 166

Gajan, S. and Kutter, B. L. (2009), "Contact interface model for shallow foundations subjected to combined cyclic loading", Journal of Geotechnical and Geoenvironmental Engineering, ASCE, Vol. 135 (3), pp 407-419

Gazetas, G. and Mylonakis, G. (1998). "Seismic Soil-Structure Interaction: New Evidence and Emerging Issues" State of the Art Paper, Geotechnical Earthquake Engineering and Soil dynamics Geo-Institute ASCE Conference, Seattle, August 3-6, 1998, Vol. II, pp. 1116-1174

Hosseinzadeh, N.A. and Nateghi, F. A. (2003), " Shake Table Study on Soil-FoundationStructure Interaction Effects", International Institute of Earthquake Engineering and Seismology , Tehran, Iran

Iai, S. (1989). "Similitude for Shaking Table Tests on Soil-Structure-Fluid Model in $1 \mathrm{~g}$ Gravitational Field," Soils and Foundations, JSSMFE, 29(1), 105-118.

Iai, S. (1999). "Soil-structure interaction studies through shaking table tests." Proceedings of the Second International Conference, 927-940.

Itasca Consulting Group, Inc. (Itasca). (2005). FLAC3D: "Fast Lagrangian analysis of continua in 3 dimensions, version 3.0." User's manual, Minneapolis. 
Khalil, L., \& Sadek, M. (2007). "Influence of the soil - structure interaction on the fundamental period of buildings." Earthquake Engineering and Structural Dynamics, (36), 2445-2453.

Kim, S., and Stewart, J. P., (2003). "Kinematic soil-structure interaction from strong motion recordings," J. Geotech. Geoenviron. Eng. 129 (4), 323-335.

Kline, S. (1965). Similitude and Approximation Theory, McGraw-Hill, New York.

Kokusyo, T. and Iwatate, T. (1979), "Scaled Model Vibration Tests and Numerical Analysis on Nonlinear Dynamic Response of Soft Ground", Journal of Structural Mechanics and Earthquake Engineering, pp285

Kramer, S. (1996). “Geotechnical Earthquake Engineering.” Prentice Hall.

Langhaar, H. (1951). Dimensional Analysis and Theory of Models, John Wiley and Sons, New York.

Lin, A.N. and Jennings, P.C. (1984). "Effect of embedment on foundation-soil impedances," J. Engrg. Mech., ASCE, 110(7), 1060-1075.

Luco, J.E. (1980). "Linear soil-structure interaction," in Soil-structure interaction: The status of current analysis methods and research, J.J. Johnson, ed., Rpt. No. NUREG/CR1780 and UCRL-53011, U.S. Nuclear Regulatory Com., Washington DC and Lawrence Livermore Lab., Livermore, CA

Luco, J.E. and Wong, H.L. (1986). "Response of a rigid foundation to a spatially random ground motion,” J. Earthquake Engrg. Struct. Dynamics, 14(6), 891-908.

Luco, J.E. and Mita, A. (1987). "Response of circular foundation to spatially random ground motions," J. Engrg. Mech., ASCE, 113(1), 1-15.

Lysmer, J., Udaka, T., Tsai, C. F., and Seed, H. B. (1975). "FLUSH: a computer program for approximate 3-D analysis of soil-structure interaction problems."

Lysmer, J.m Tabatabie-Raisi, M., Tajirian, F., Ostadan, F. (1981) SASSI - a system for analysis of soil-structure interaction, Report No. UCB/GT/81-02, University of California Berkeley, CA

Mazzoni, S., Mckenna, F., Scott, M.H., and Fenves, G.L. (2000), “OpenSees User Manual," Version 2, Pacific Earthquake Engineering Research Centre, University of California, Berkeley.

Meymand, P. J. (1998). "Shaking table scale model tests of nonlinear soil-pilesuperstructure interaction in soft clay." Ph.D. Dissertation, Univ. of California, Berkeley. 
Mita, A. and Luco, J.E. (1989). "Dynamic response of a square foundation embedded in an elastichalfspace," Soil Dynamics and Earthquake Engrg., 8(2), 54-67.

Moncarz, P. and Krawinkler, H. (1981). "Theory and Application of Experimental Model Analysis in Earthquake Engineering,” Rpt. No. 50, John Blume Earthquake Eng. Ctr., Stanford Univ.

Moss, R. E. S., Crosariol, V., and Kuo, S. (2010). "Shake table testing to quantify seismic soil-structure-interaction of underground structures." 5th International Conference on Recent Advances in Geotechnical Earthquake Engineering and Soil Dynamics, San Diego, May 24-29

Mylonakis, G, and Gazetas, G. (2000). "Seismic soil-structure interaction: Beneficial or detrimental?" Journal of Earthquake Engineering 4(3): 277-301.

Paolucci, R., Shirato, M., and Yilmaz, T.M.. (2007). "Seismic behaviour of shallow foundations $\square$ : Shaking table experiments vs numerical modelling." Earthquake (November 2007): 577-595.

Pitilakis, D., Dietz, M., Wood, D. M., Clouteau, D., \& Modaressi, A. (2008). "Numerical simulation of dynamic soil-structure interaction in shaking table testing." Soil Dynamics and Earthquake Engineering, 28(6), 453-467. doi:10.1016/j.soildyn.2007.07.011

Pitilakis, D., \& Clouteau, D. (2009). "Equivalent linear substructure approximation of soil-foundation-structure interaction: model presentation and validation." Bulletin of Earthquake Engineering, 8(2), 257-282.

Preisig, M. and Jeremic, B. (2005) "Nonlinear Finite Element Analysis of Dynamic SoilFoundationStructure Interaction" Department of Civil and Environmental Engineering, University of California, Davis, Ver.16.

Randolph, M. F., and Houlsby, G. T. (1984). "Limiting pressure on a circular pile loaded laterally in cohesive soil." Geotechnique, 34(4), 613-623.

Rayhani, M. H. T., and M. H. El Naggar. (2008). "Numerical Modeling of Seismic Response of Rigid Foundation on Soft Soil." International Journal of Geomechanics 8(6): 336.

Resendiz, D. and Roesset, J.M. (1987). "Soil-Structure Interaction in Mexico City During the 1985 Earthquake", The Mexico Earthquakes -1985, ASCE special publication

Rocha, M. (1957). "The Possibility of Solving Soil Mechanics Problems by Use of Models," Proc. 4th Intl. Conf. Soil Mech. Fdn. Eng., London, Vol. 1, 183-188. 
Roesset, J.M. (1980). "A review of soil-structure interaction," in Soil-structure interaction: The status of current analysis methods and research, J.J. Johnson, ed., Rpt. No. NUREG/CR-1780 and UCRL-53011, U.S. Nuclear Regulatory Com., Washington DC, and Lawrence Livermore Lab., Livermore, CA.

Schnabel, P.B., Lysmer, J., and Seed, H.B. (1972). "SHAKE: A Computer Program for Earthquake Response Analysis of Horizontally Layered Soil Deposits", Rpt. No. UCB/EERC-72/12,Earthquake Engrg. Research Ctr., Univ. of California; 1991 modifications by Idriss, I.M. and Sun,J.I.

Seed, H.B. and Lysmer, J. (1980). "The seismic soil-structure interaction problem for nuclear facilities," in Soil-structure interaction: the status of current analysis methods and research, J.J. Johnson, ed., Rpt. No. NUREG/CR-1780 and UCRL-53011, U.S. Nuclear Regulatory Com., Washington DC and Lawrence Livermore Lab., Livermore, CA.

Seed, R.B., Dickenson, S.E., Riemer, M.F., Bray, J.D., Sitar, N., Mitchell, J.K., Idriss, I.M., Kayen,R.E., Kropp, A.K., Harder, L.F. Jr., and Power, M.S. (1990). "Preliminary report on the principal geotechnical aspects of the October 17, 1989 Loma Prieta Earthquake", Rpt. No. UCB/EERC-90/05, Earthquake Engrg. Research Ctr., Univ. of California, Berkeley.

Shirato M, Kouno T, Asai R, Nakani S, Fukui J, Paolucci R. (2008). “Large-scale experiments on nonlinear behavior of shallow foundations subjected to large earthquakes." Soils and Foundations, Vol 48-5-5-48, pg. 673-692

Stewart, D. P., and Randolph, M. F. (1991). "A new site investigation tool for the centrifuge." Centrifuge 91 (eds. Ko and McLean), Balkema, Rotterdam, 531-538.

Stewart, J. P., and Stewart, A. F. (1997). "Analysis of soil-structure interaction effects on building response from earthquake strong motion recordings at 58 sites.' Rep. No. UCB/EERC 97/01, Earthquake Engineering Research Center, Univ. of California, Berkeley, Calif., 742.

Stewart, J.P., Seed, R.B. and Fenves, G.L. (1998). "Empirical evaluation of inertial soilstructure interaction effects," Rpt. No. PEER-98/07, Pacific Earthquake Engineering Research Center, University of California, Berkeley, 205 pgs.

Stewart, J. P., Fenves, G. L., and Seed, R. B. (1999a). "'Seismic soil-structure-interaction in buildings. I: Analytical methods.'” J. Geotech.Geoenviron. Eng., 125(1), 26-37.

Stewart, J. P., Seed, R. B., and Fenves, G. L. (1999b). "Seismic soil-structure-interaction in buildings. II: Empirical findings.”' J. Geotech.Geoenviron. Eng., 125(1), 38-48.

Takewaki, I, Takeda, N. and Uetani, K.,(2003). "Fast practical evaluation of soilstructure interaction of embedded structures." Soil Dynamics and Earthquake Engineering 23(3): 13-20 
Todorovska, M.I. (1992)." Effects of the depth of the embedment on the system response during building-soil interaction," Soil Dynamics and Earthquake Engineering, Volume 11 , Issue 2

Veletsos, A.S. and Meek, J.W. (1974). "Dynamic behavior of building-foundation systems," J.Earthquake Engrg. Struct. Dynamics, 3(2), 121-138.

Veletsos, A.S. and Wei, Y.T. (1971). "Lateral and rocking vibrations of footings," J. Soil Mech. and Foundations Div., ASCE, 97(9), 1227-1248.

Veletsos, A.S. and Verbic, B. (1973). "Vibration of viscoelastic foundations," J. Earthquake Engrg. Struct. Dynamics, 2(1), 87-102.

Veletsos, A.S. and Nair V.V. (1975). "Seismic interaction of structures on hysteretic foundations," J. Struct. Engrg., ASCE 101(1), 109-129.

Veletsos, A.S. and Prasad, A.M. (1989). "Seismic interaction of structures and soils: stochastic approach,” J. Struct. Engrg., ASCE, 115(4), 935-956.

Veletsos, A.S., Prasad, A.M., and Wu, W.H. (1997). “Transfer functions for rigid rectangular foundations,” J. Earthquake Engrg. Struct. Dynamics, 26 (1), 5-17.

Wolf, John P. (1985). “Response 1-16 Dynamic soil-structure interaction.” Prentice-Hall. 


\section{APPENDIX A - DISPLACEMENT DATA FROM THE WIRE POTS}

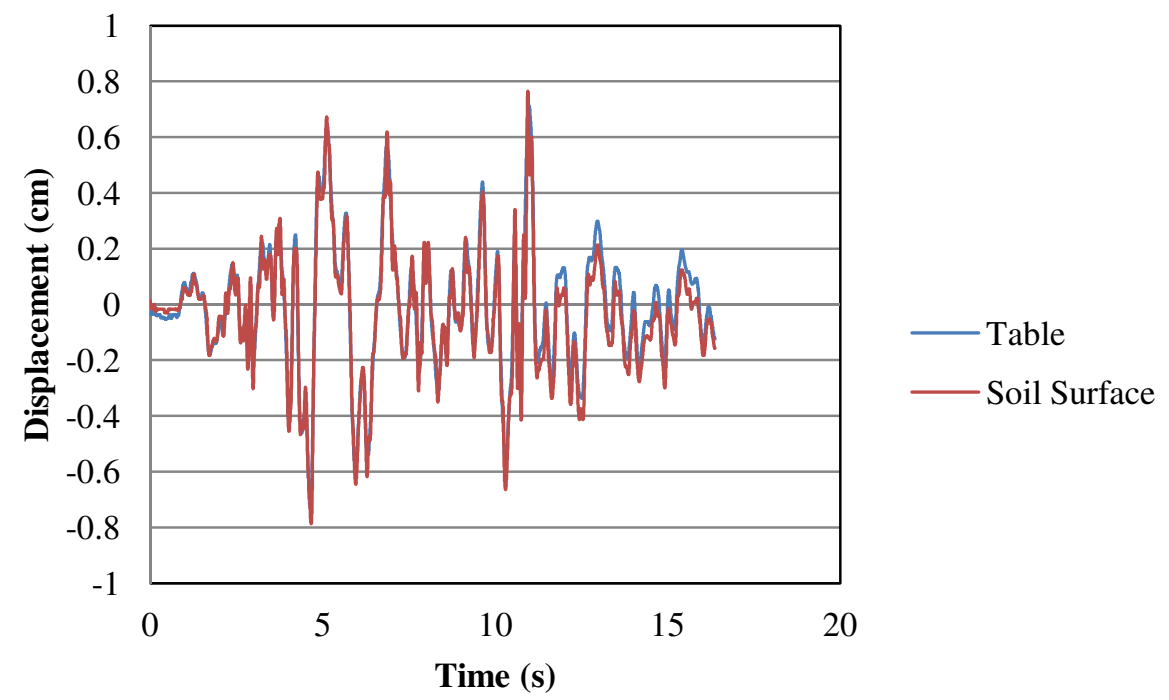

Figure A- 1 Comparison of displacement data from the string pots between the soil surface level and the shake table level for the Joshua Tree 000 motion

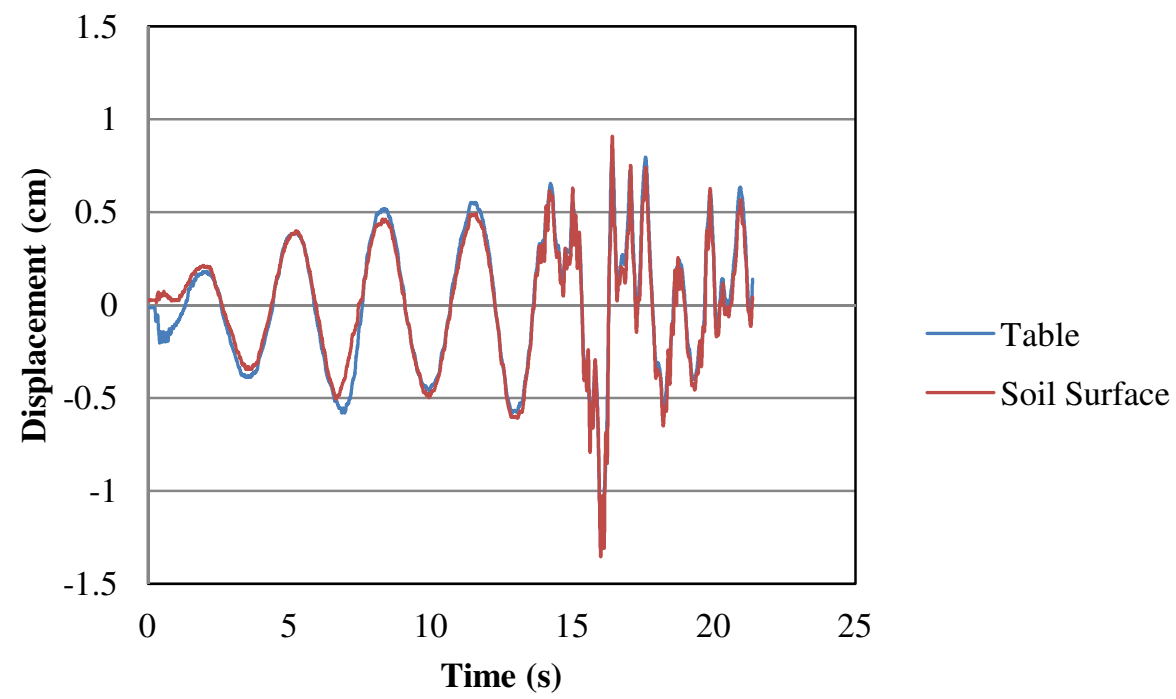

Figure A- 2 Comparison of displacement data from the string pots between the soil surface level and the shake table level for the Joshua Tree 090 motion 


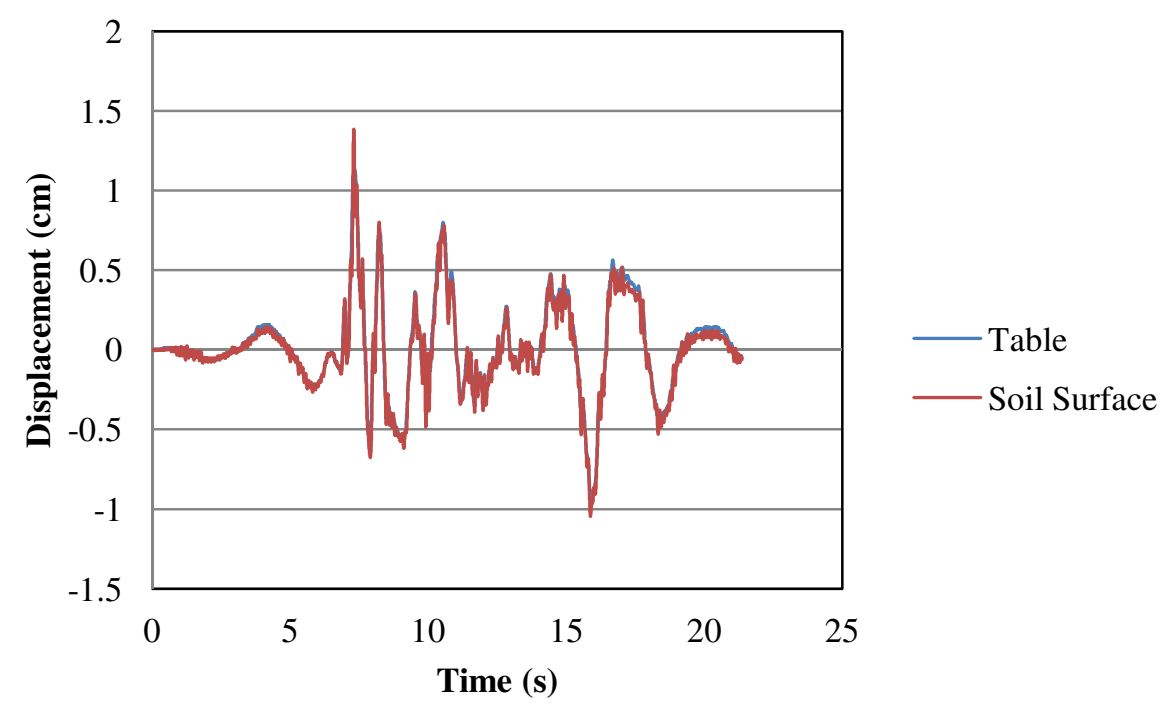

Figure A- 3 Comparison of displacement data from the string pots between the soil surface level and the shake table level for the El Centro 180 motion

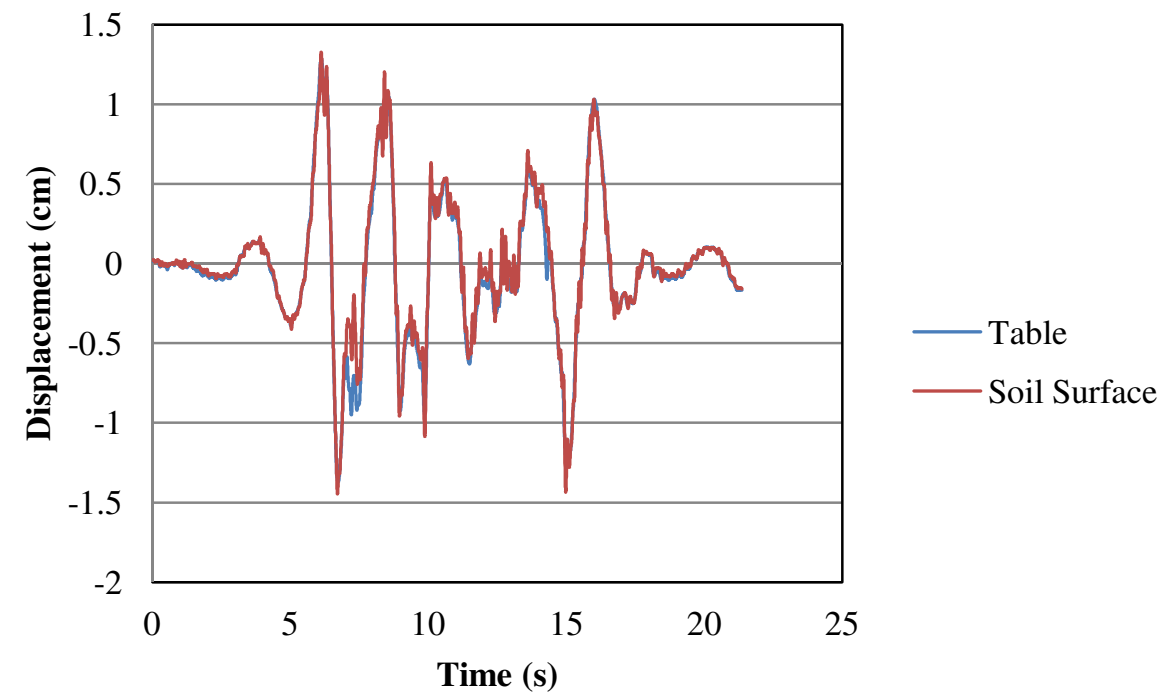

Figure A- 4 Comparison of displacement data from the string pots between the soil surface level and the shake table level for the El Centro 270 motion 


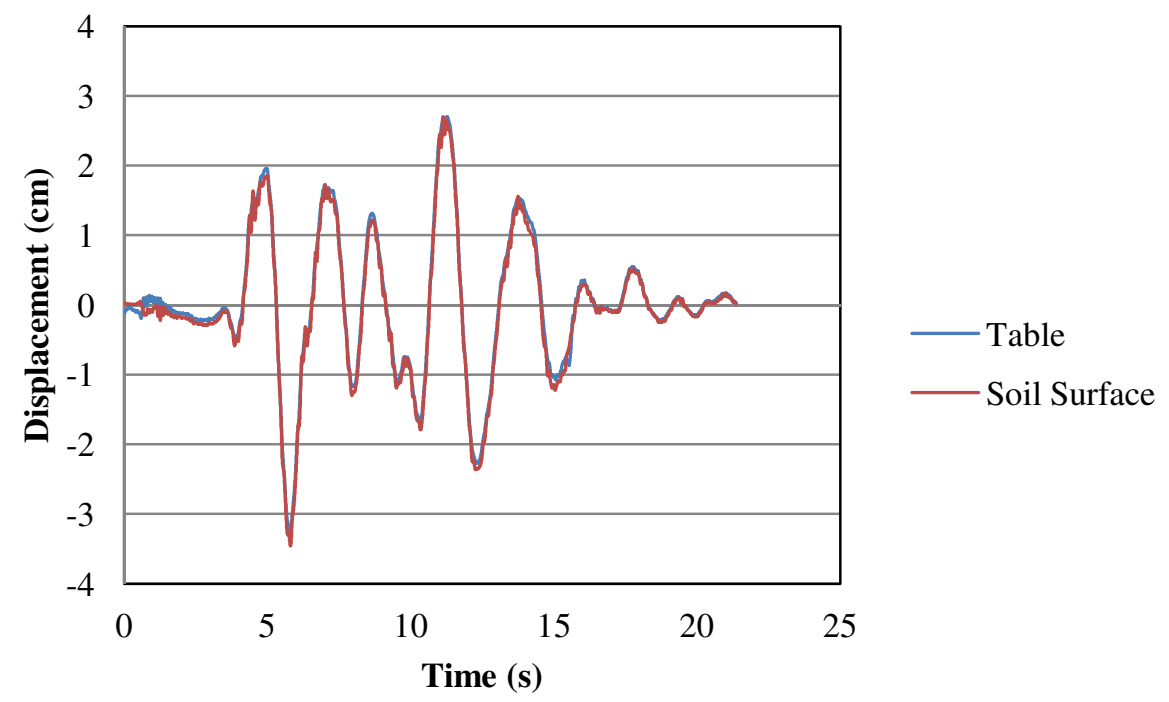

Figure A- 5 Comparison of displacement data from the string pots between the soil surface level and the shake table level for the TCU Chi Chi North motion

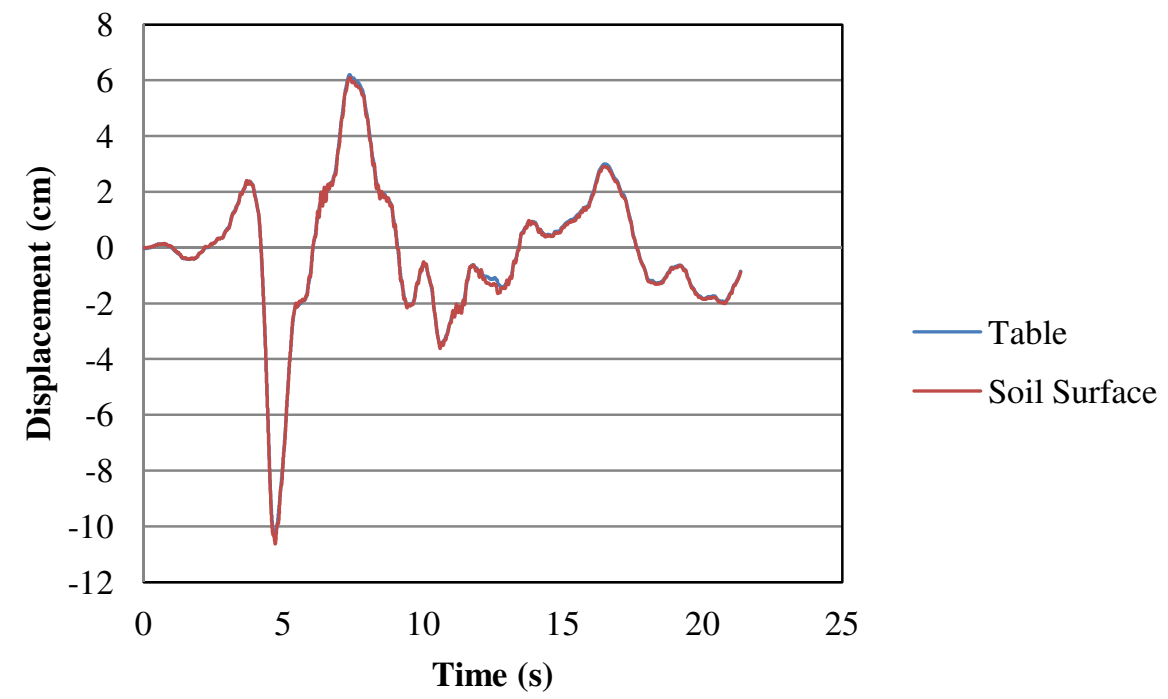

Figure A- 6 Comparison of displacement data from the string pots between the soil surface level and the shake table level for the TCU Chi Chi West motion 


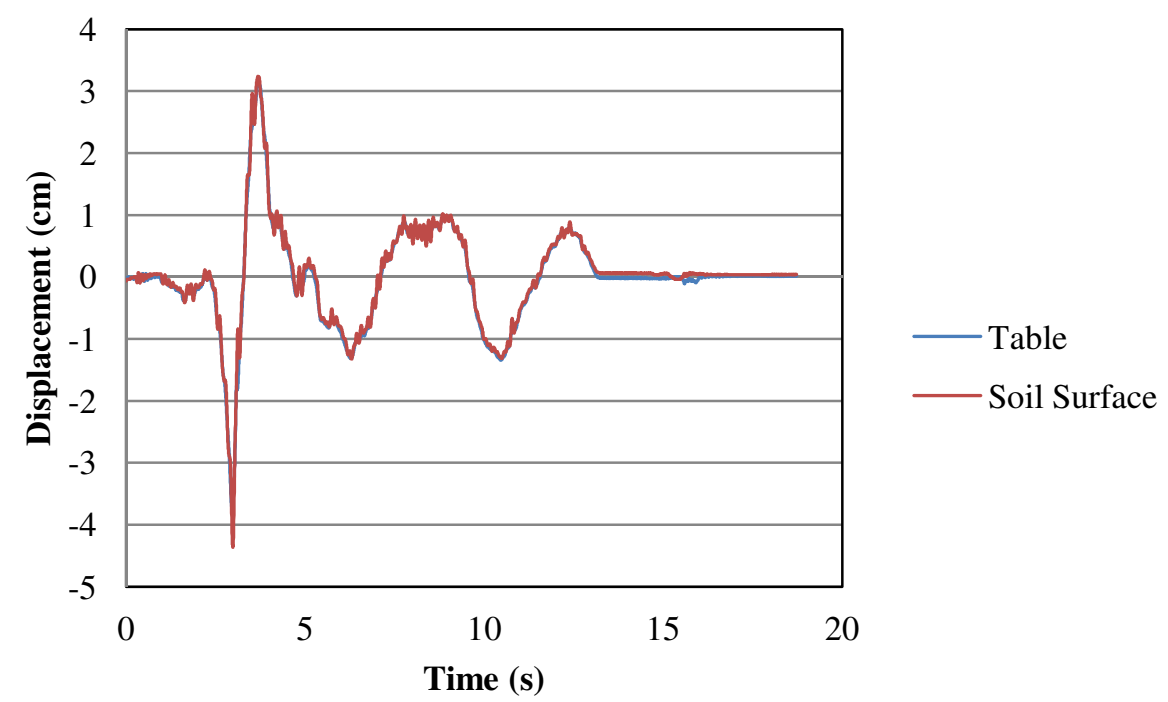

Figure A- 7 Comparison of displacement data from the string pots between the soil surface level and the shake table level for the Cape Mendocino 000 motion

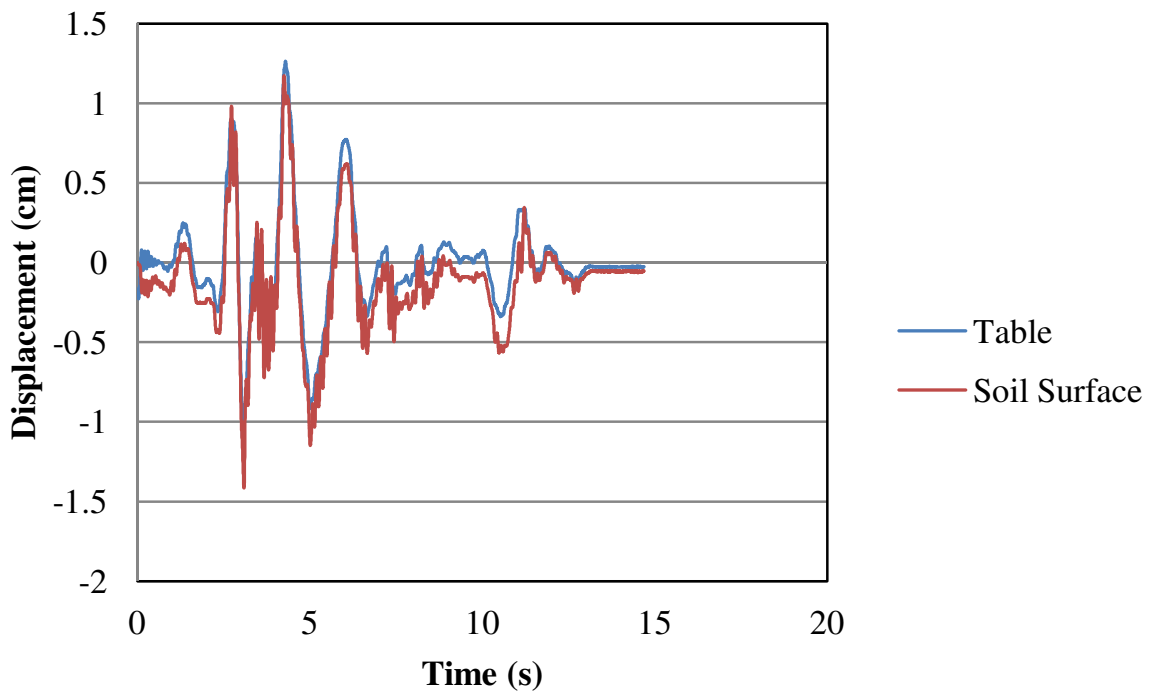

Figure A- 8 Comparison of displacement data from the string pots between the soil surface level and the shake table level for the Cape Mendocino 090 motion 


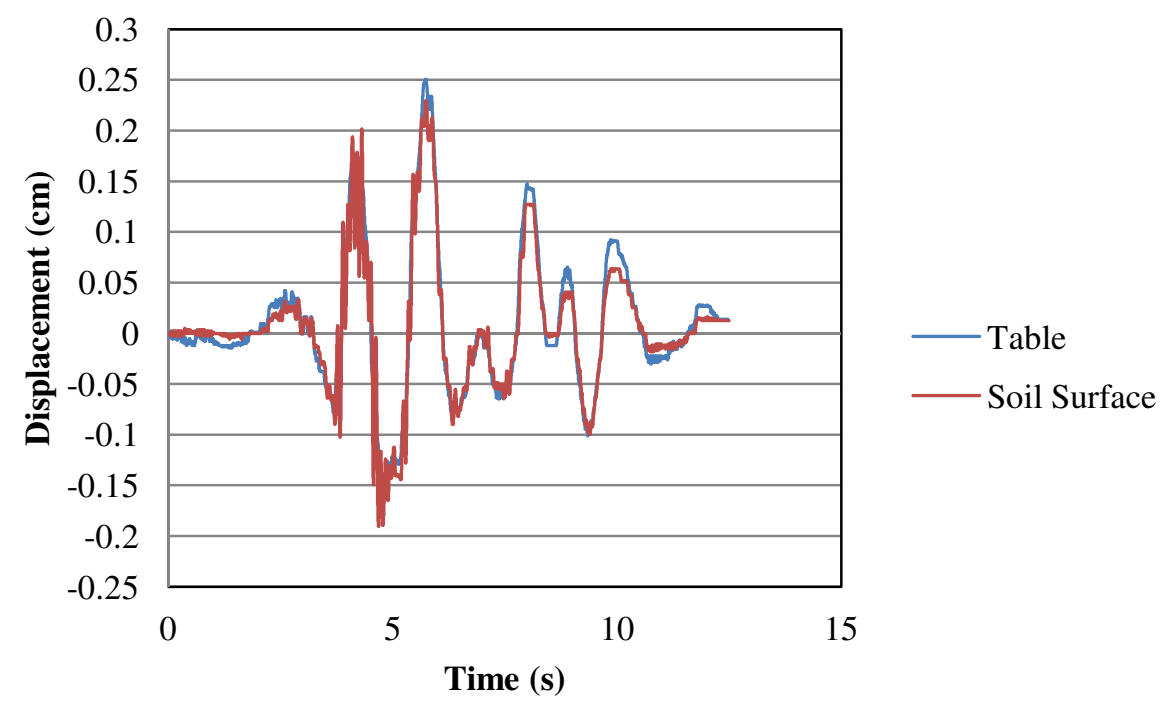

Figure A- 9 Comparison of displacement data from the string pots between the soil surface level and the shake table level for the HSUP 045 motion

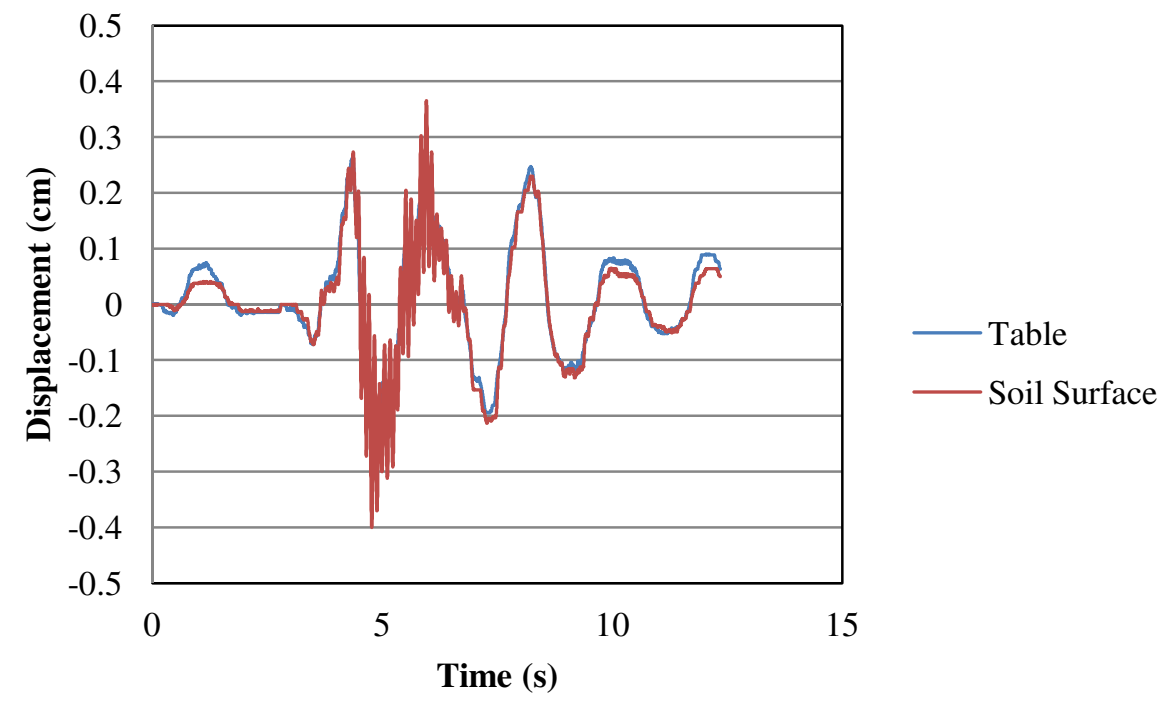

Figure A- 10 Comparison of displacement data from the string pots between the soil surface level and the shake table level for the HSUP 135 motion 


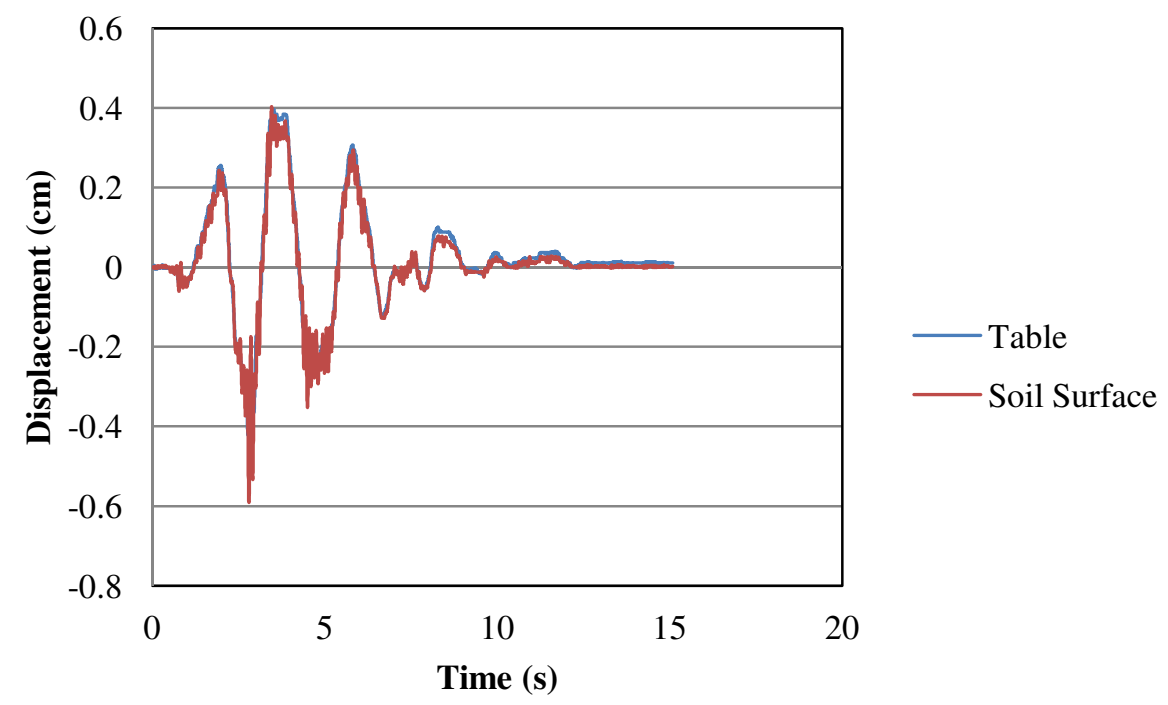

Figure A- 11 Comparison of displacement data from the string pots between the soil surface level and the shake table level for the L09 000 motion

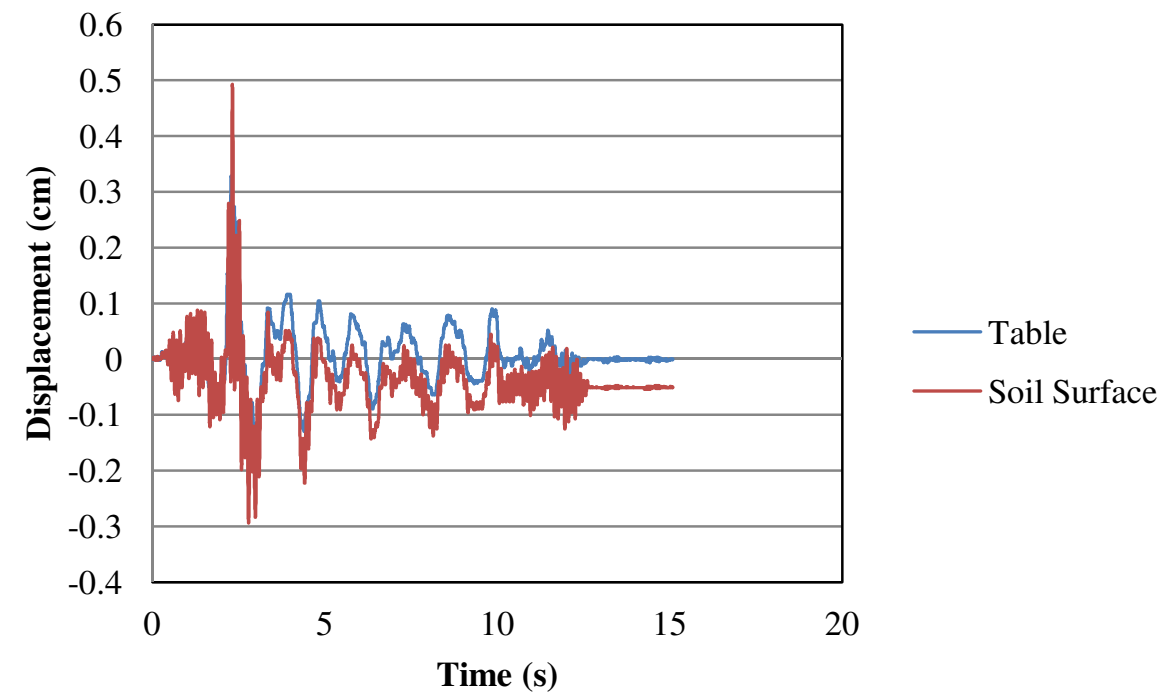

Figure A- 12 Comparison of displacement data from the string pots between the soil surface level and the shake table level for the L09 090 motion 


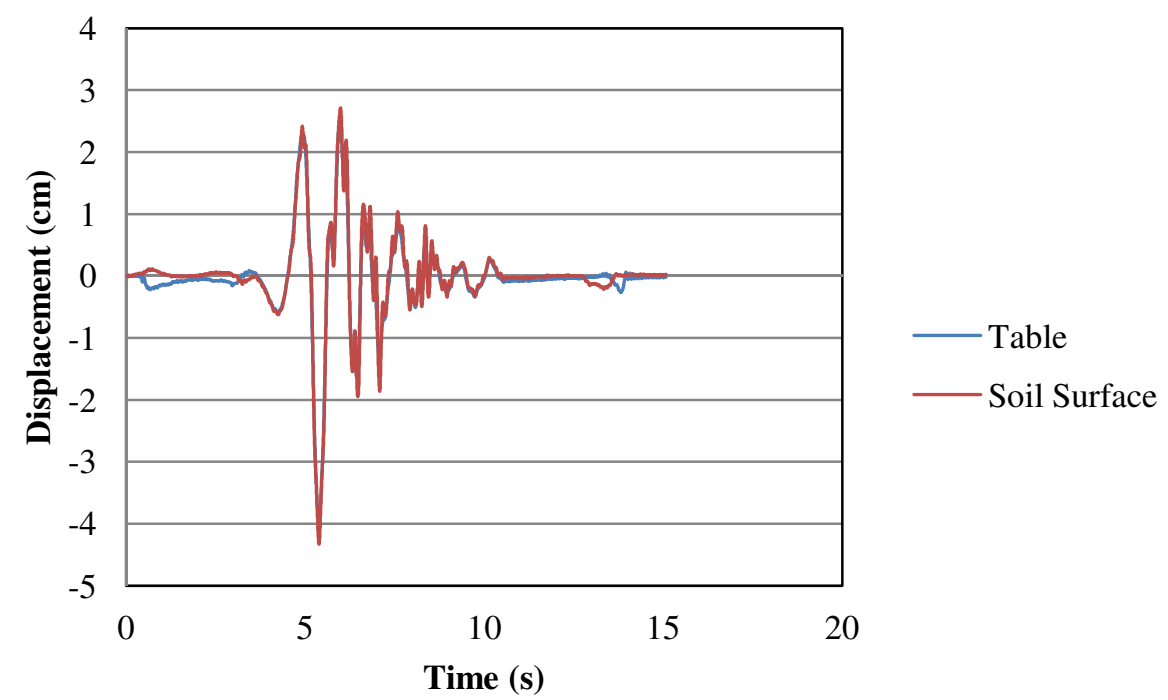

Figure A- 13 Comparison of displacement data from the string pots between the soil surface level and the shake table level for the Los Gatos 000 motion

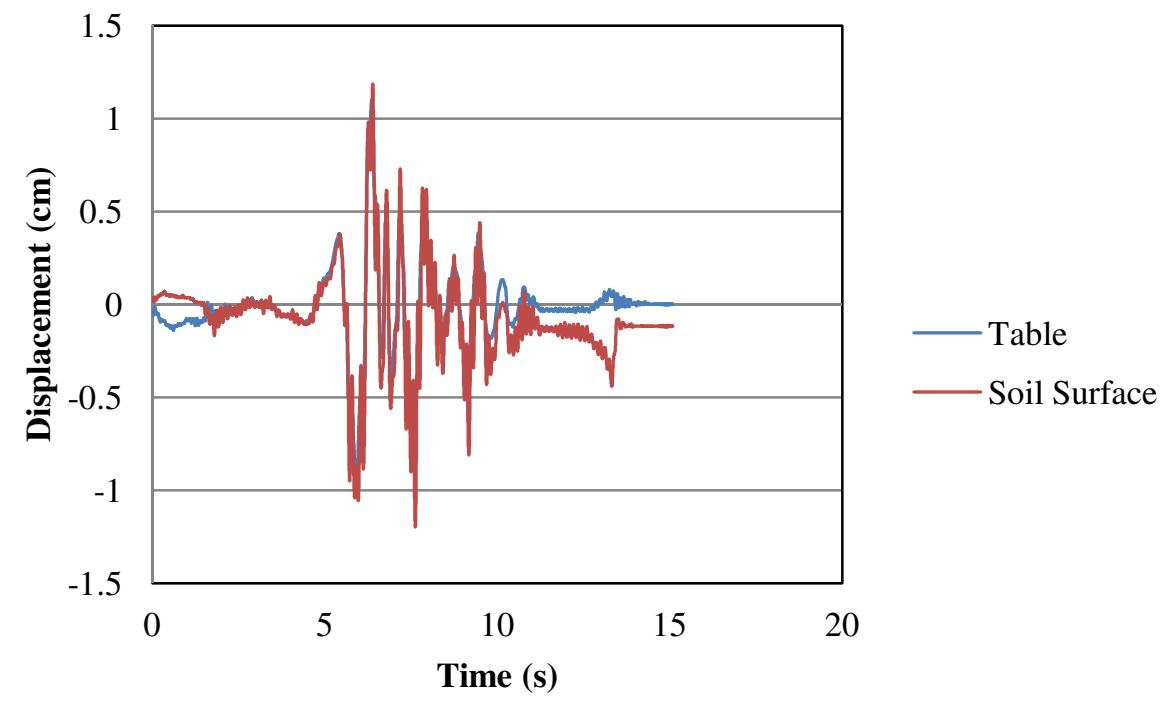

Figure A- 14 Comparison of displacement data from the string pots between the soil surface level and the shake table level for the Los Gatos 090 motion 
APPENDIX B - SPECCTRAL ACCELERATION RESULT GRAPHS 

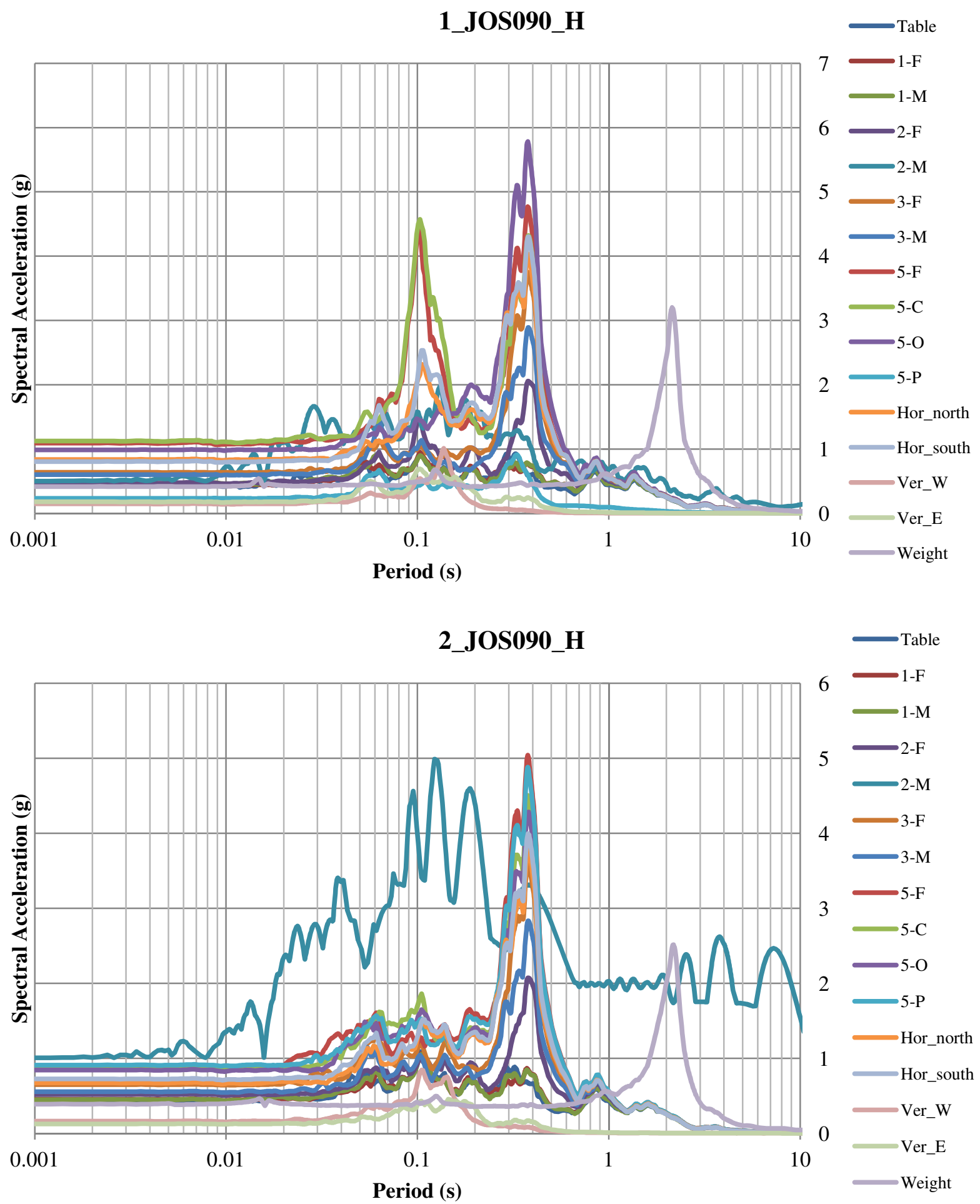

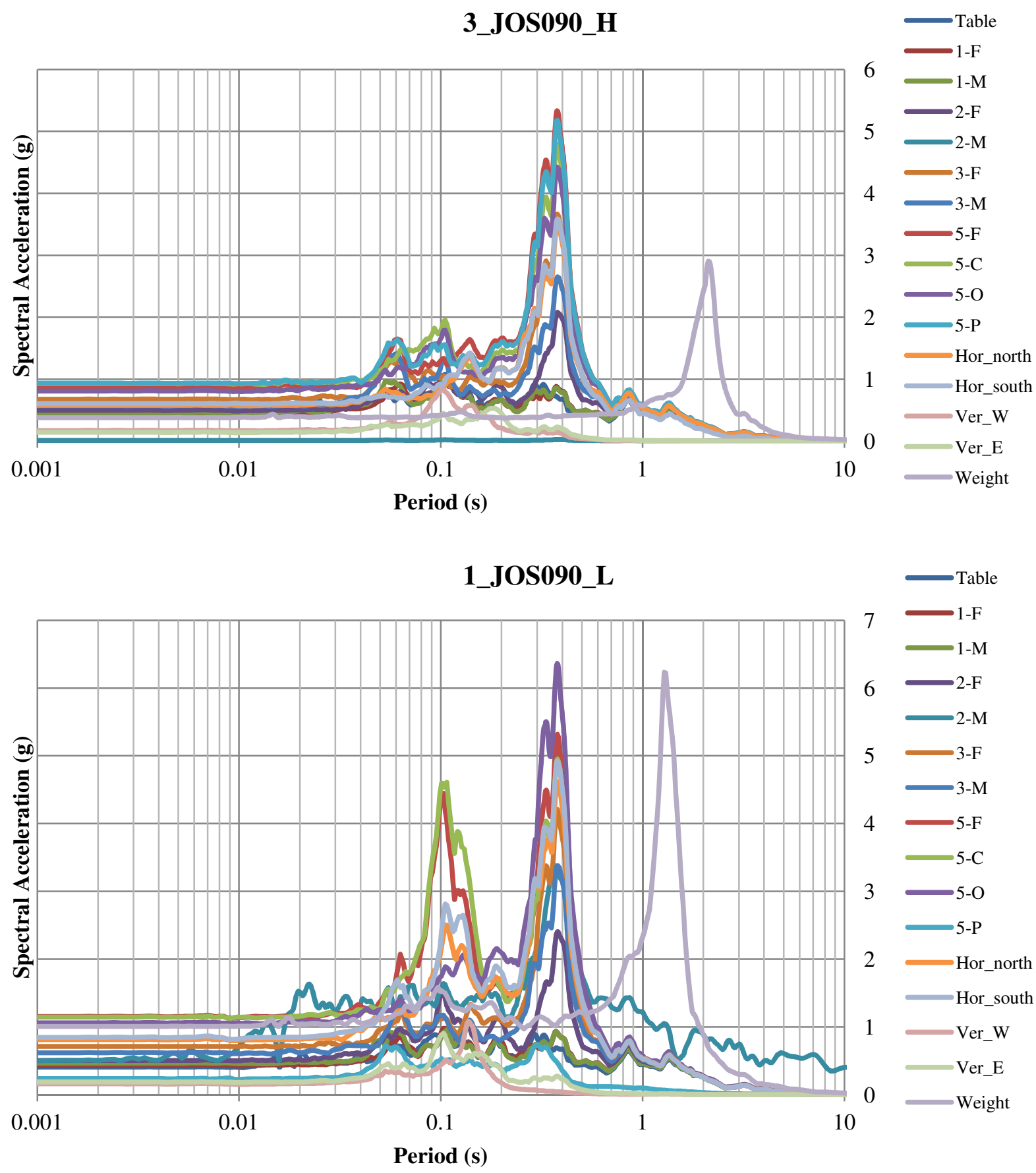


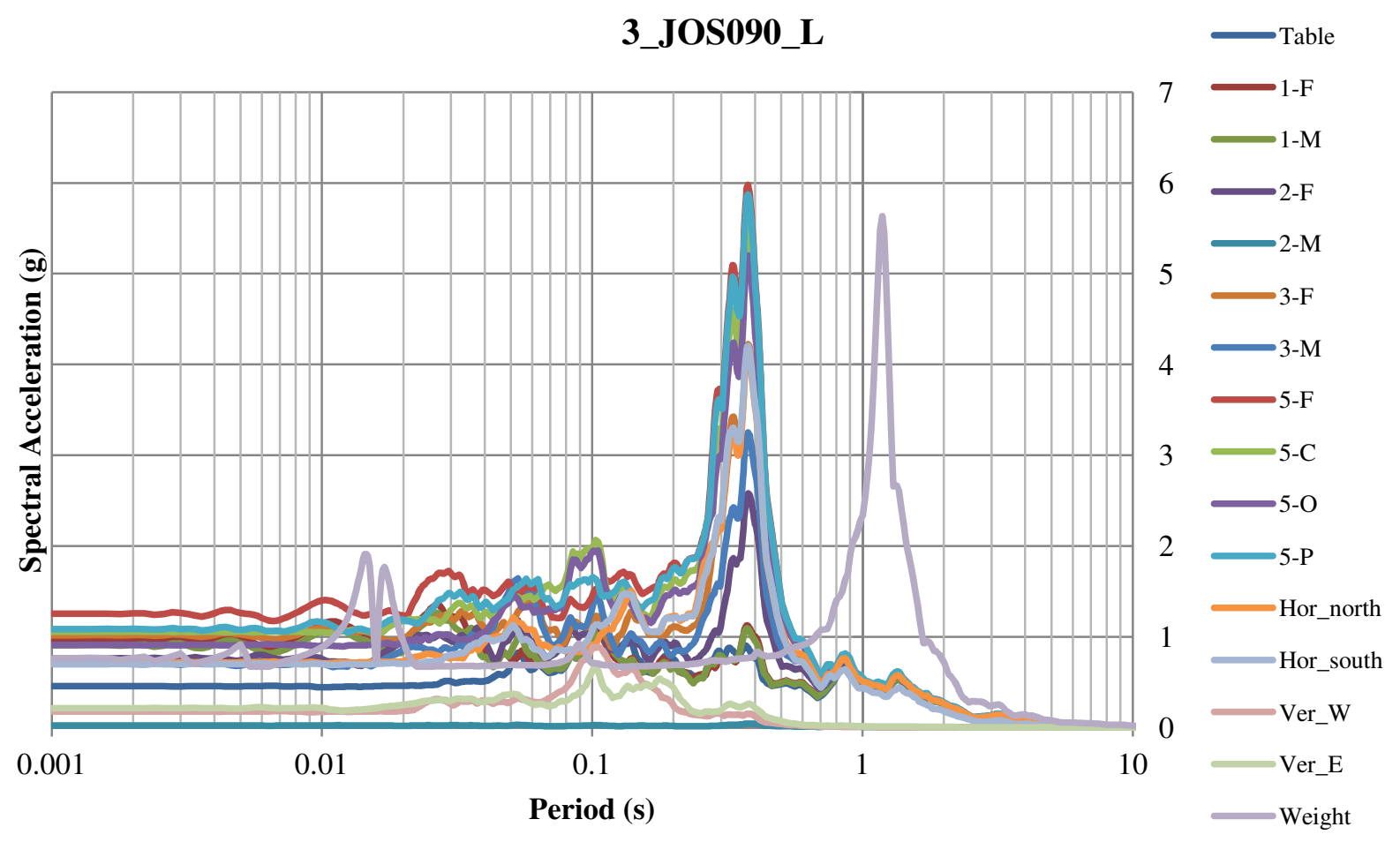



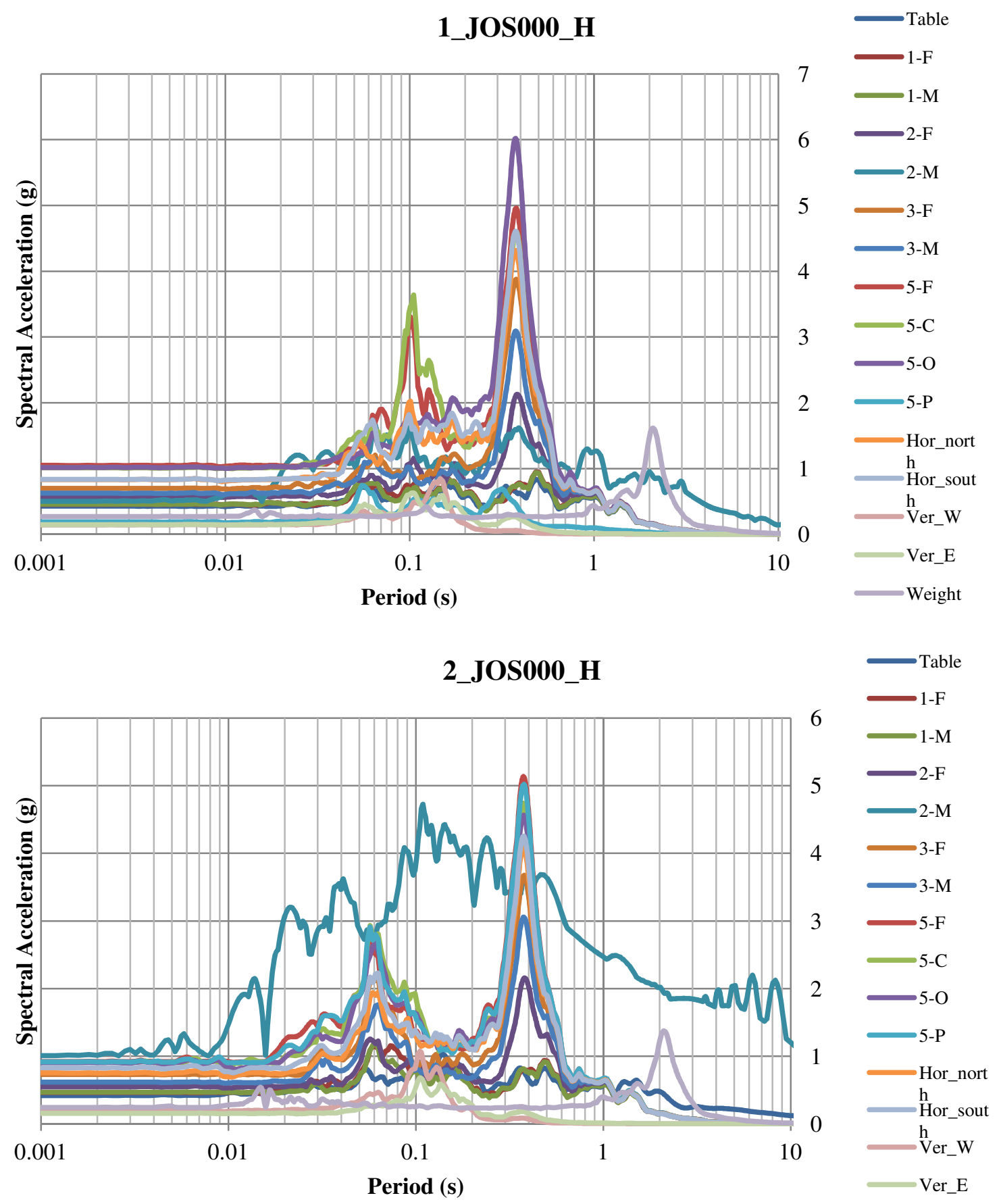

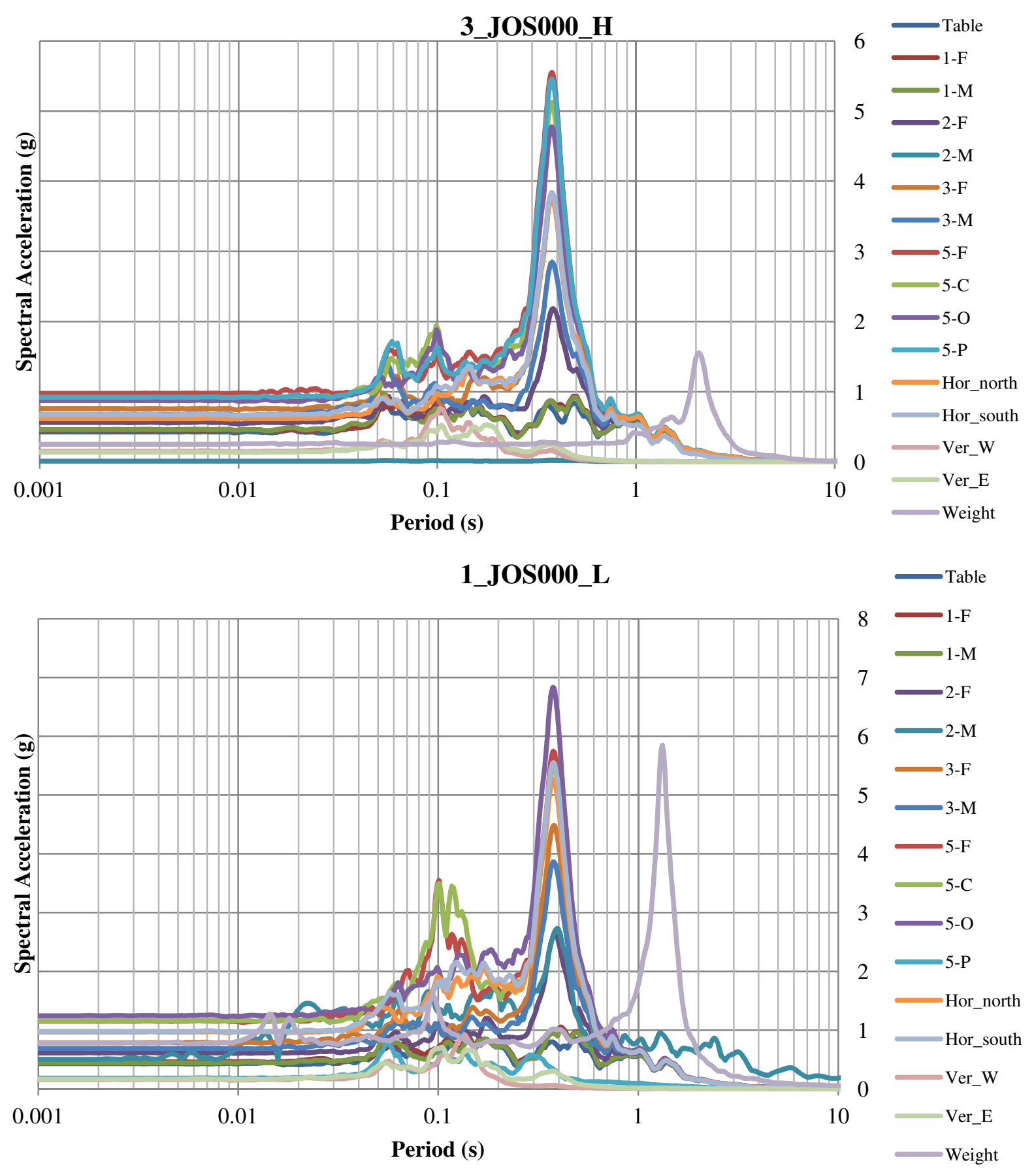
2_JOS000_L
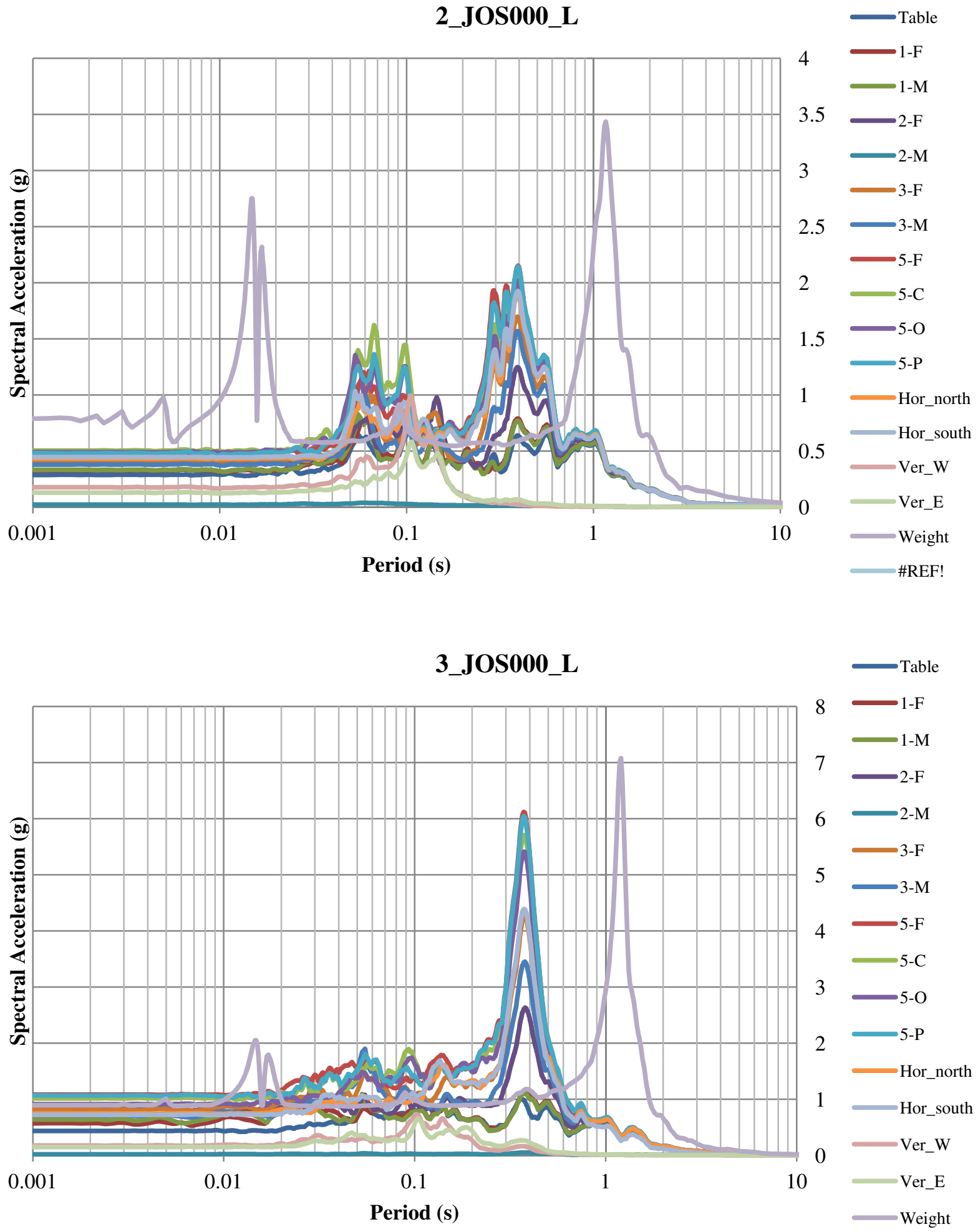

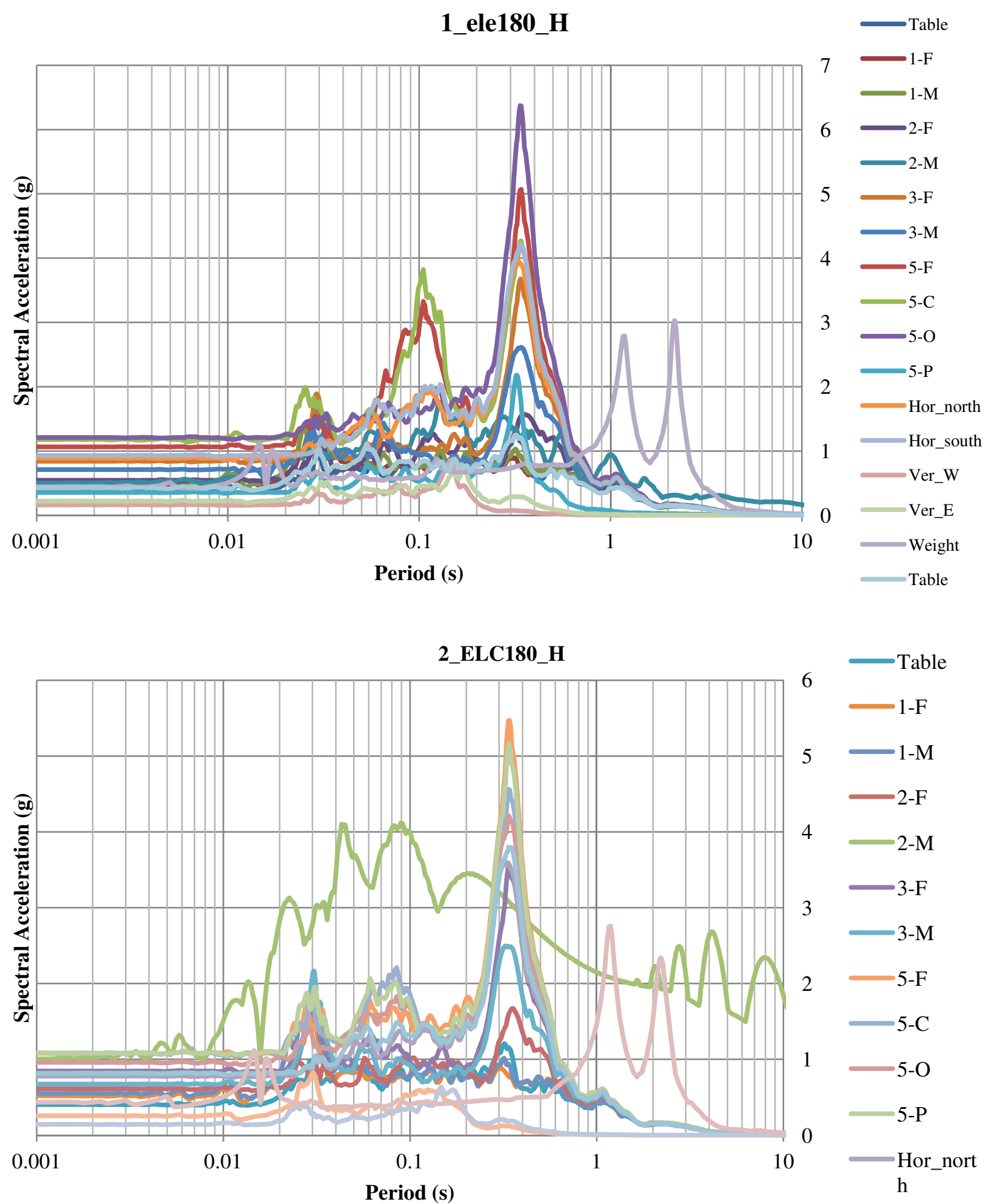

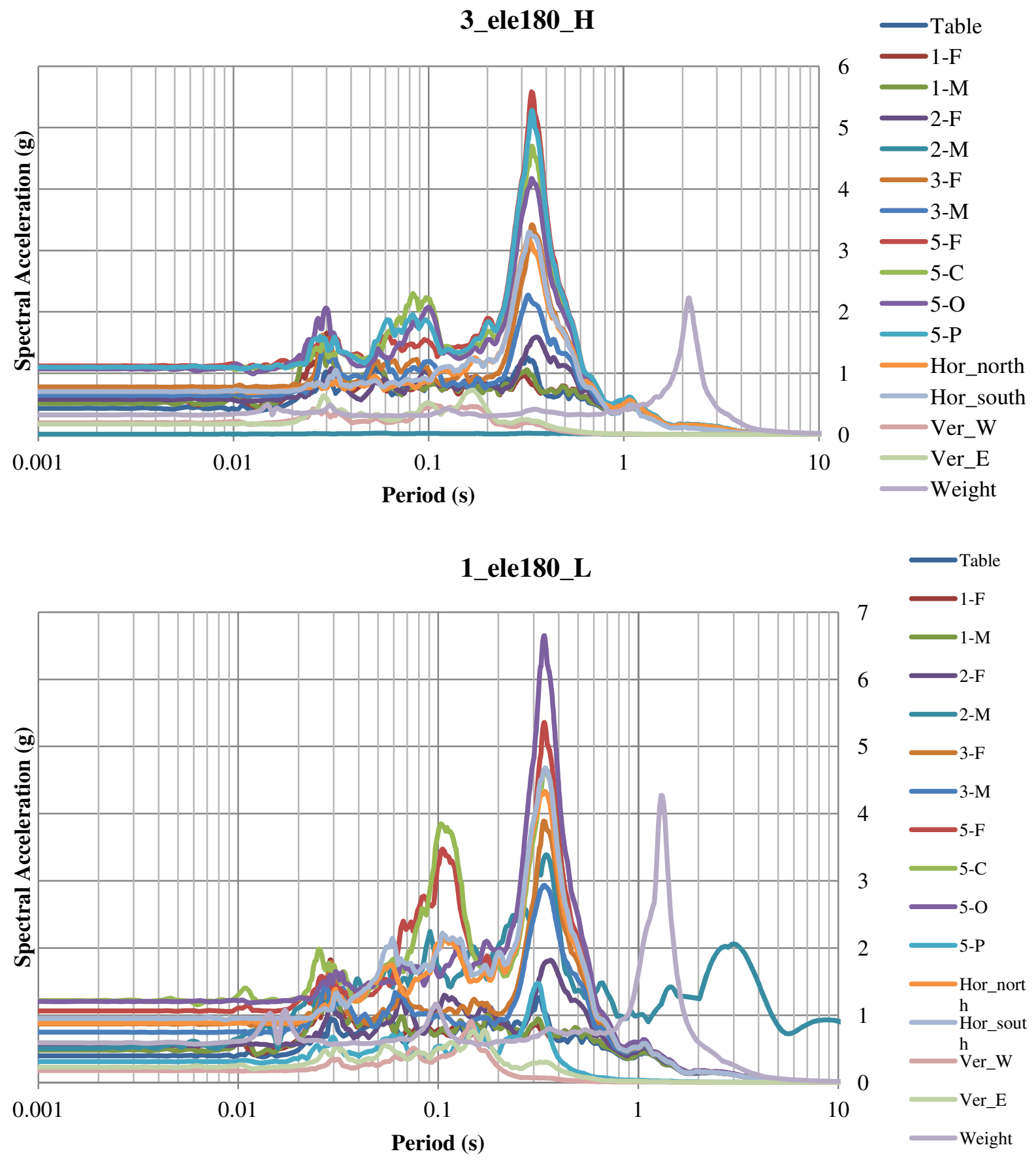

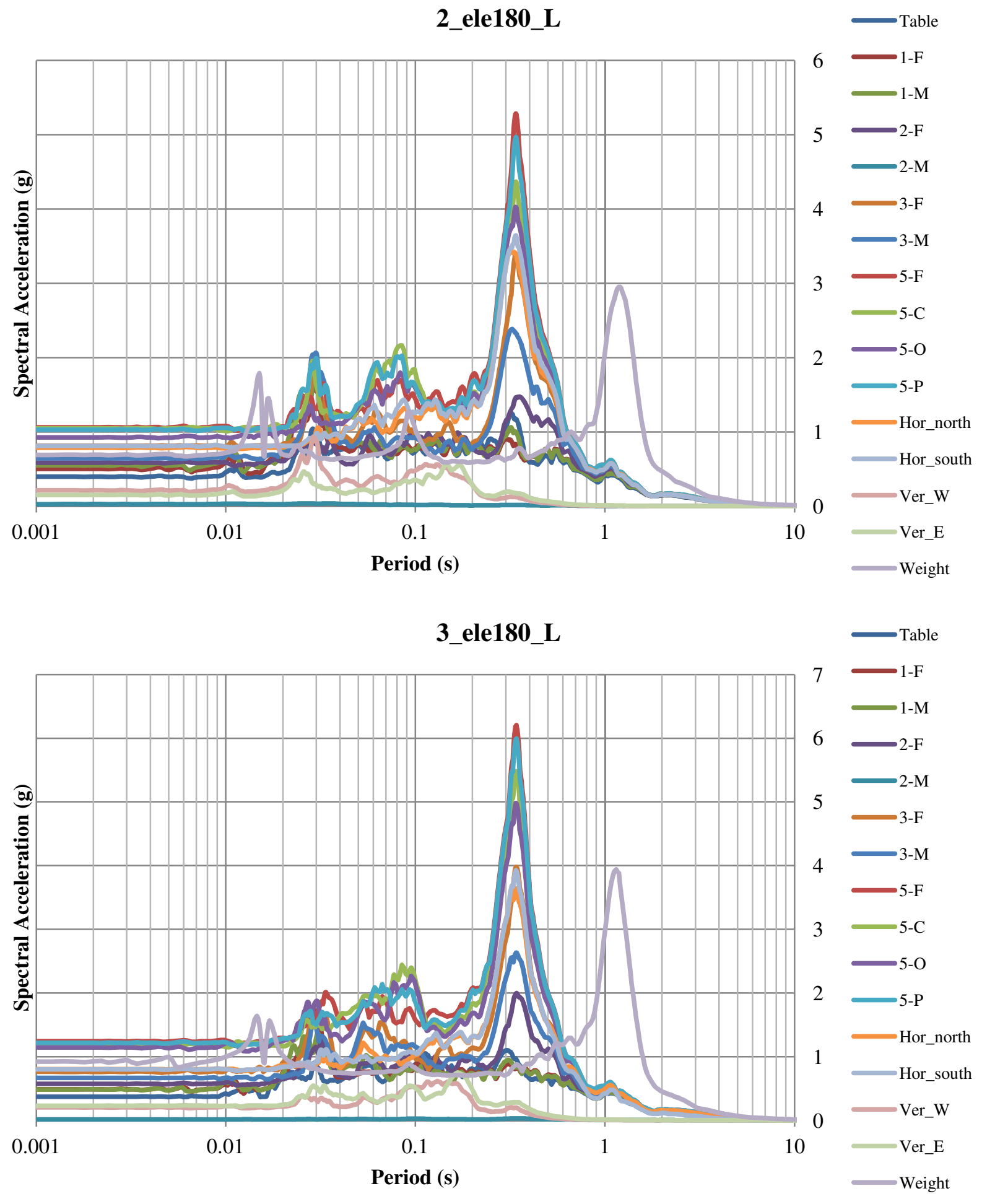

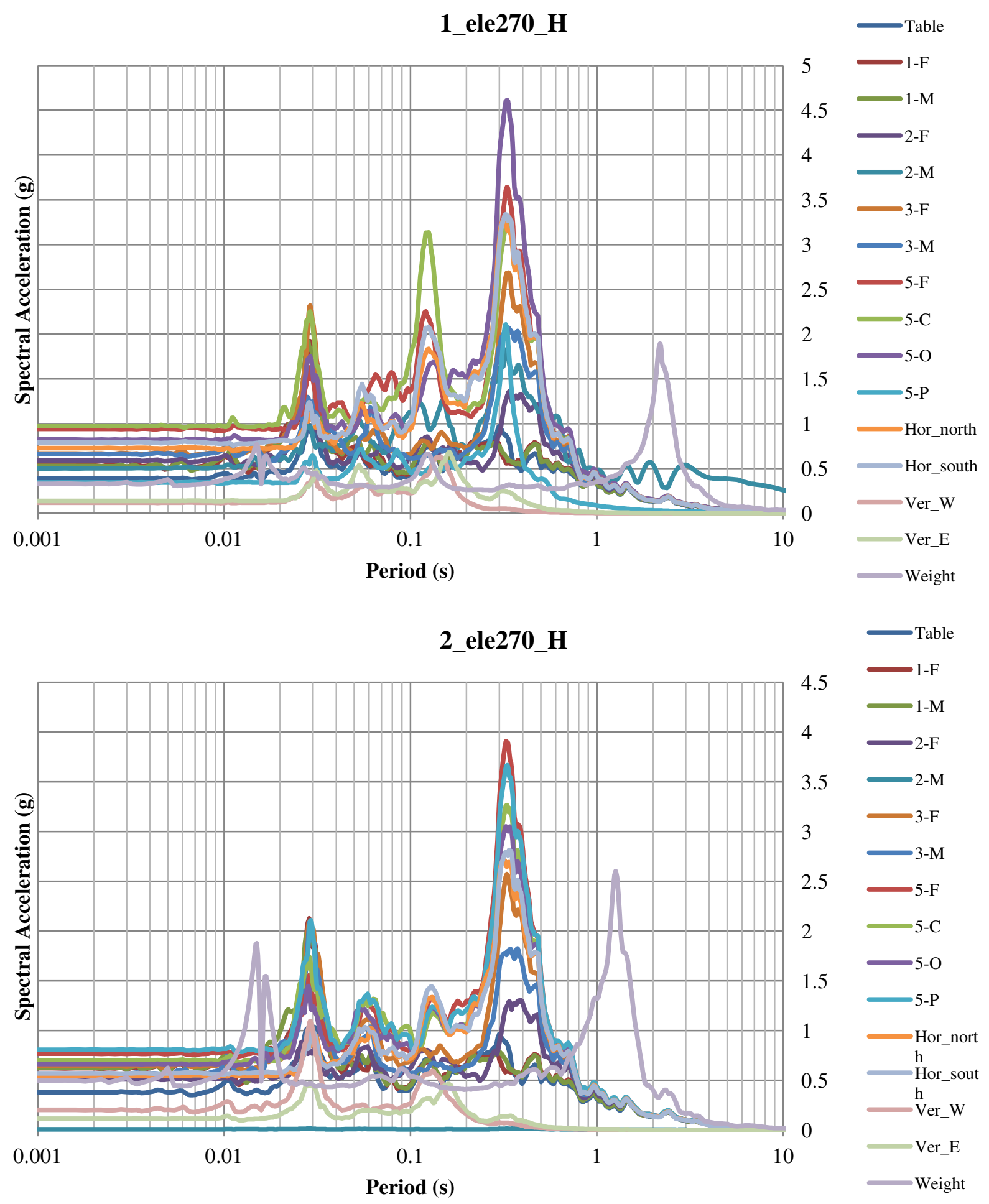

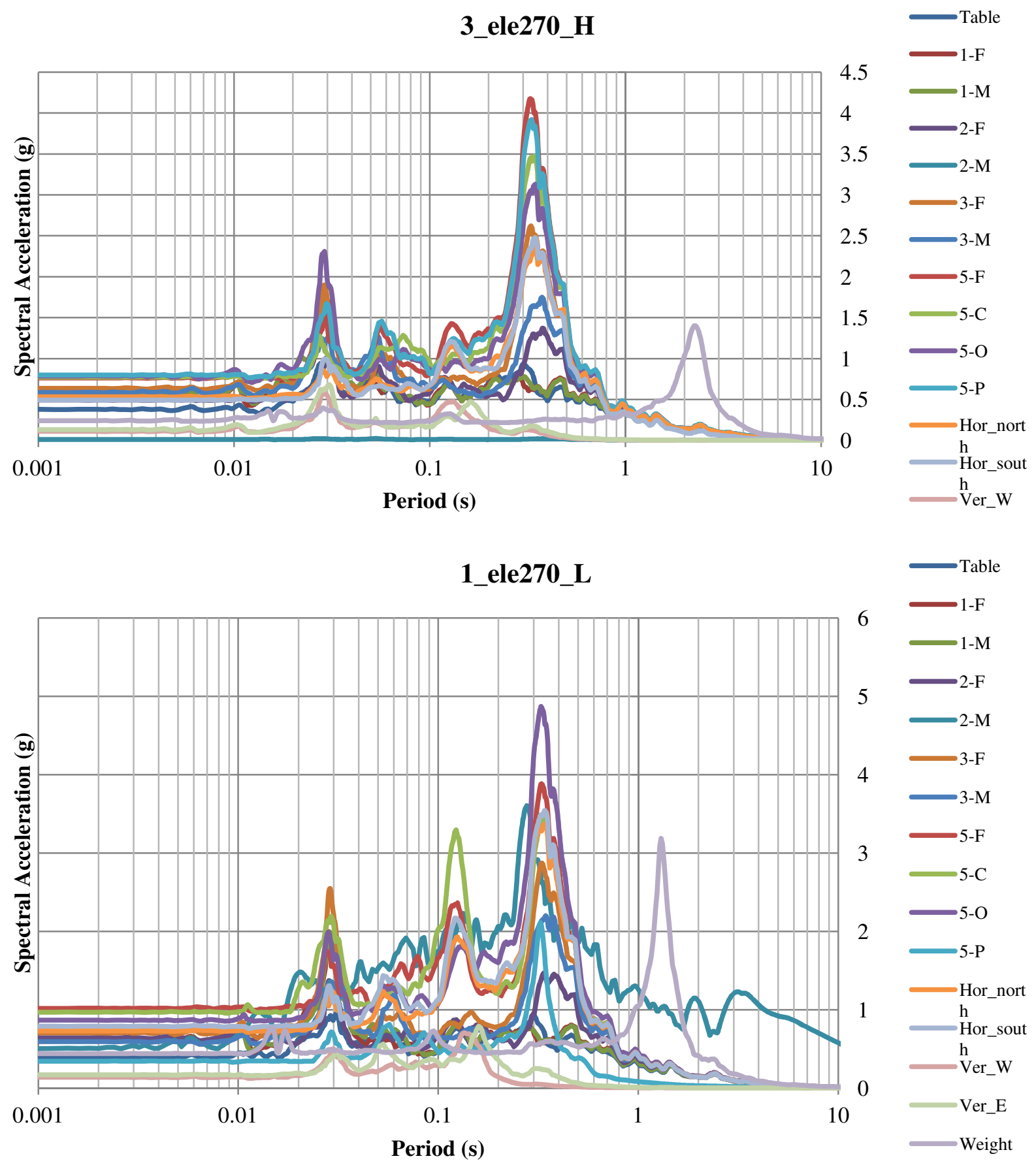


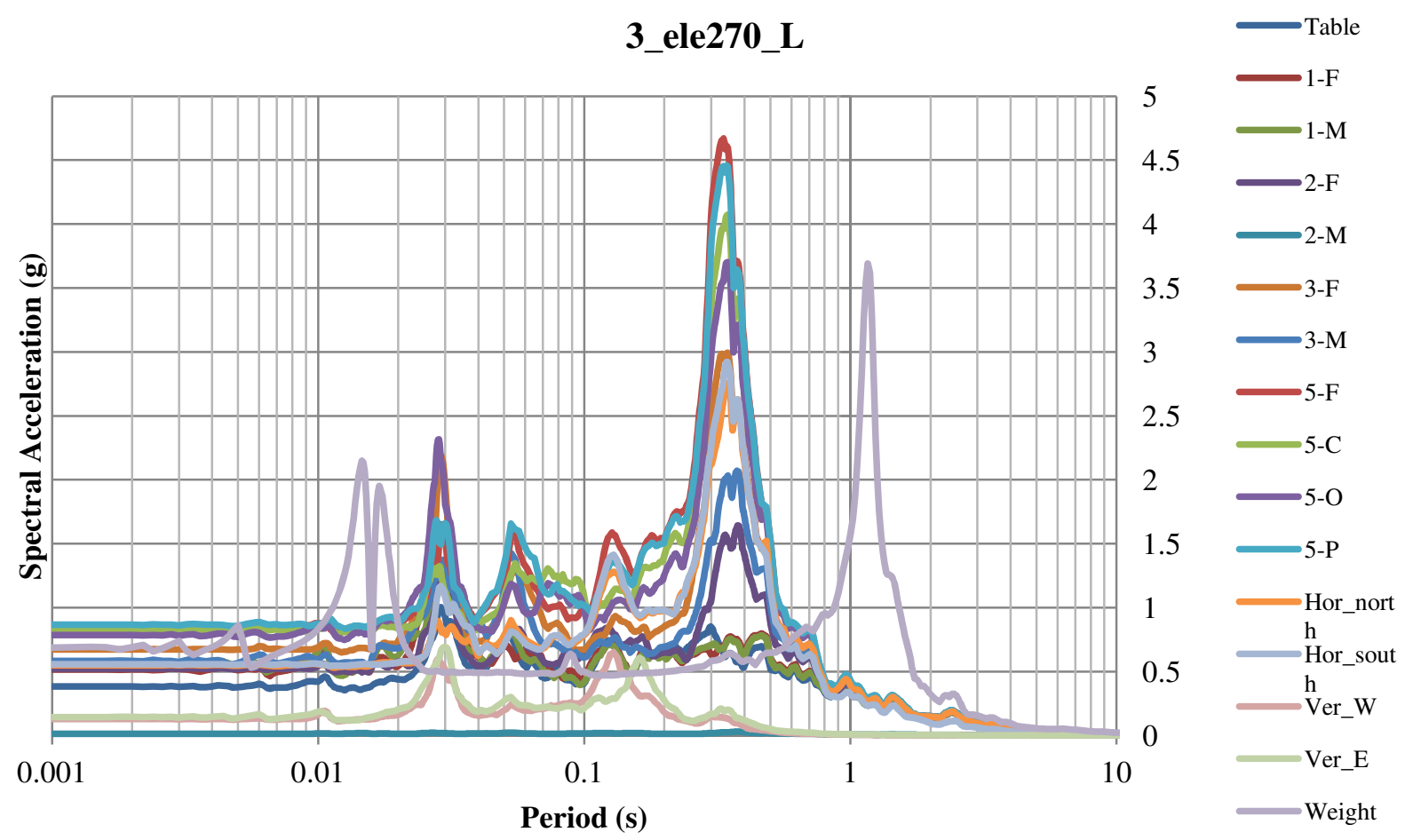



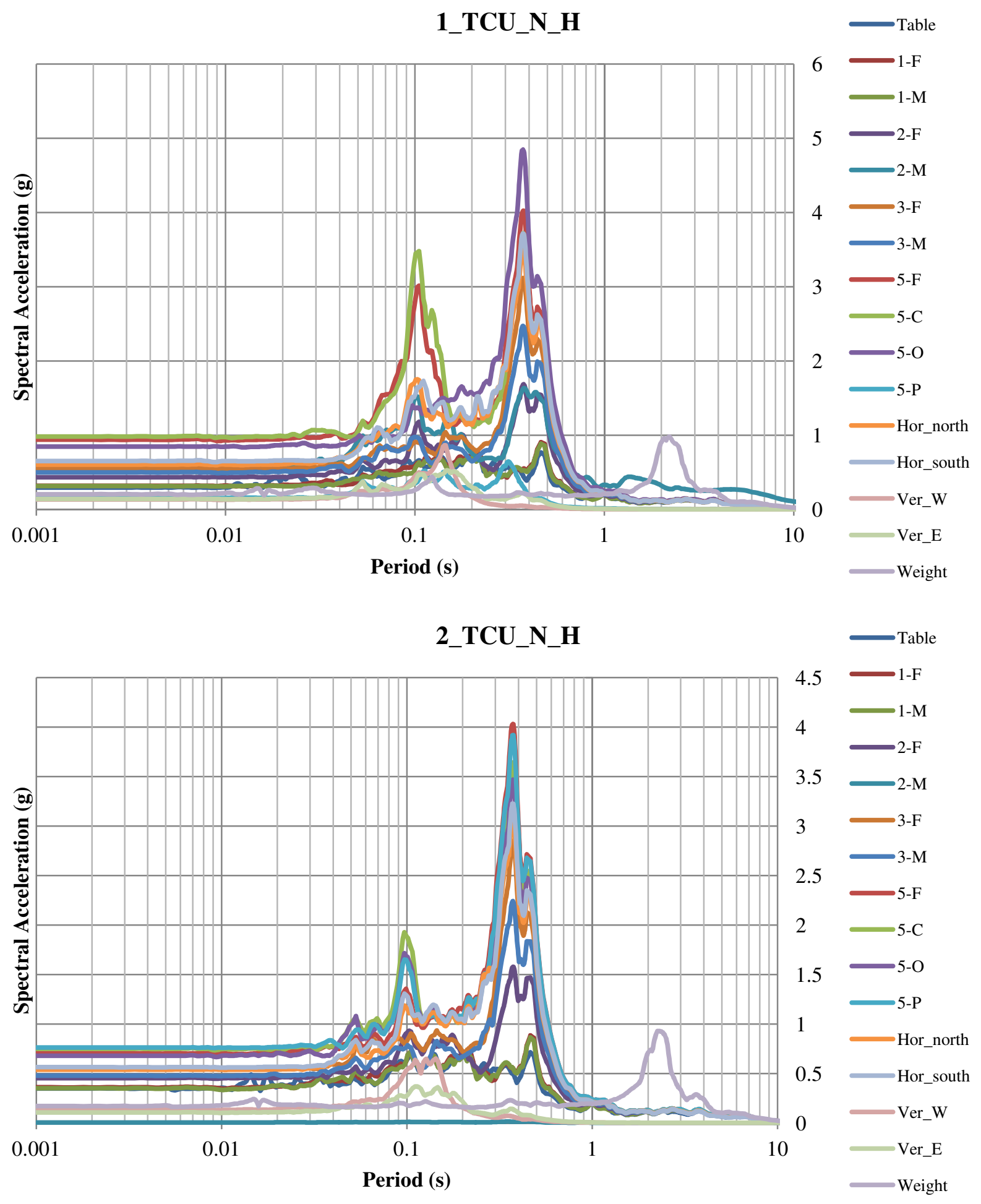

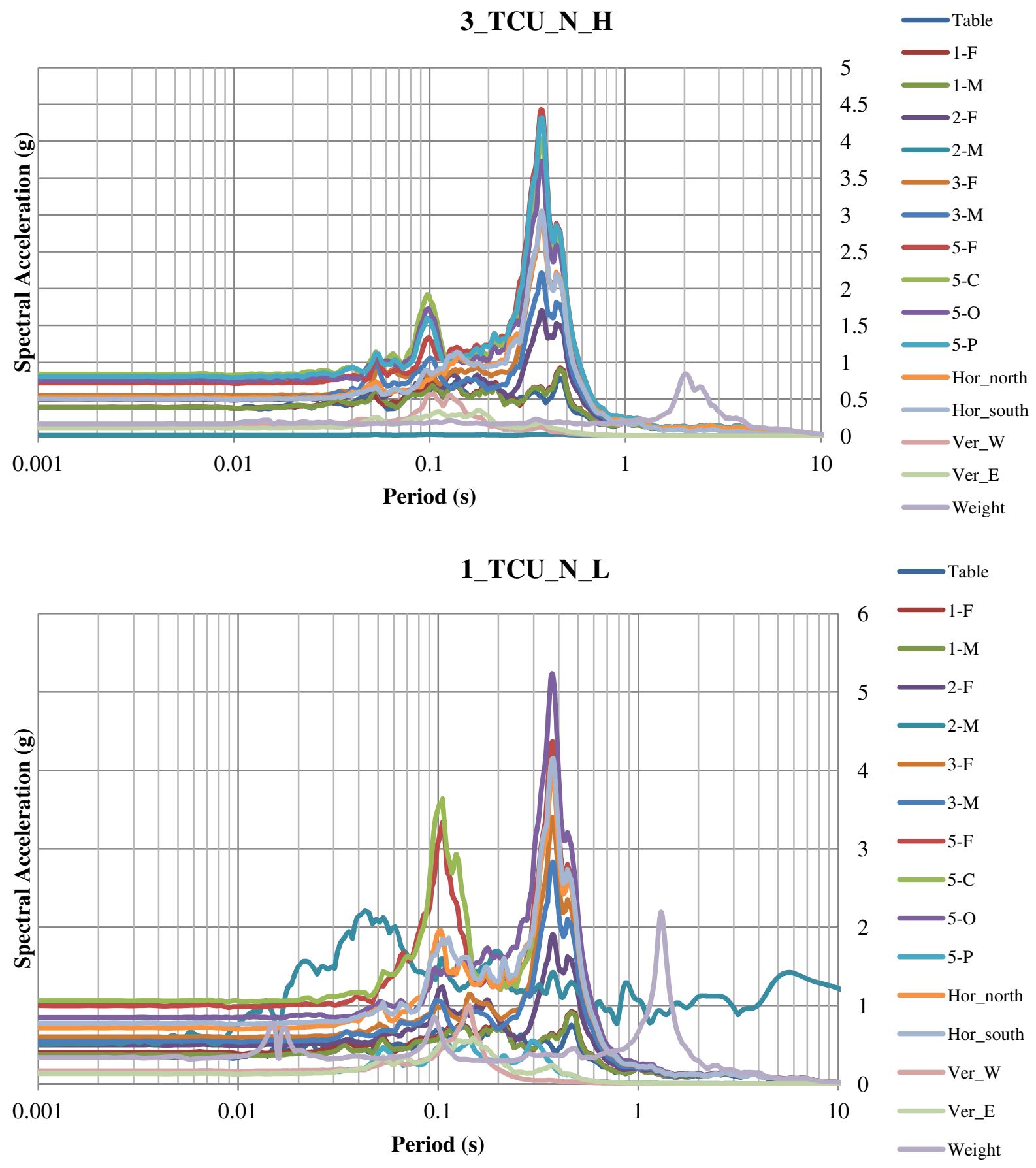

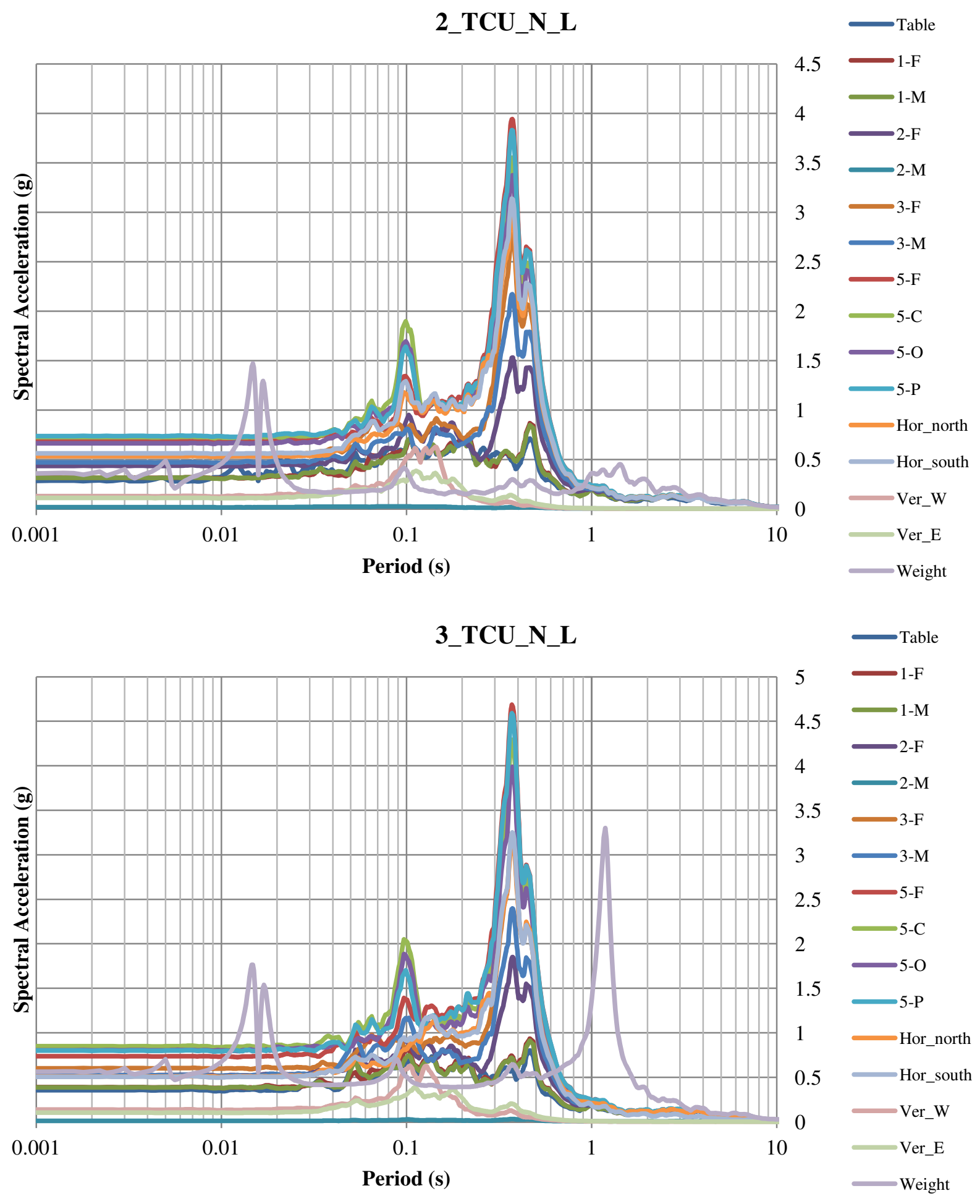


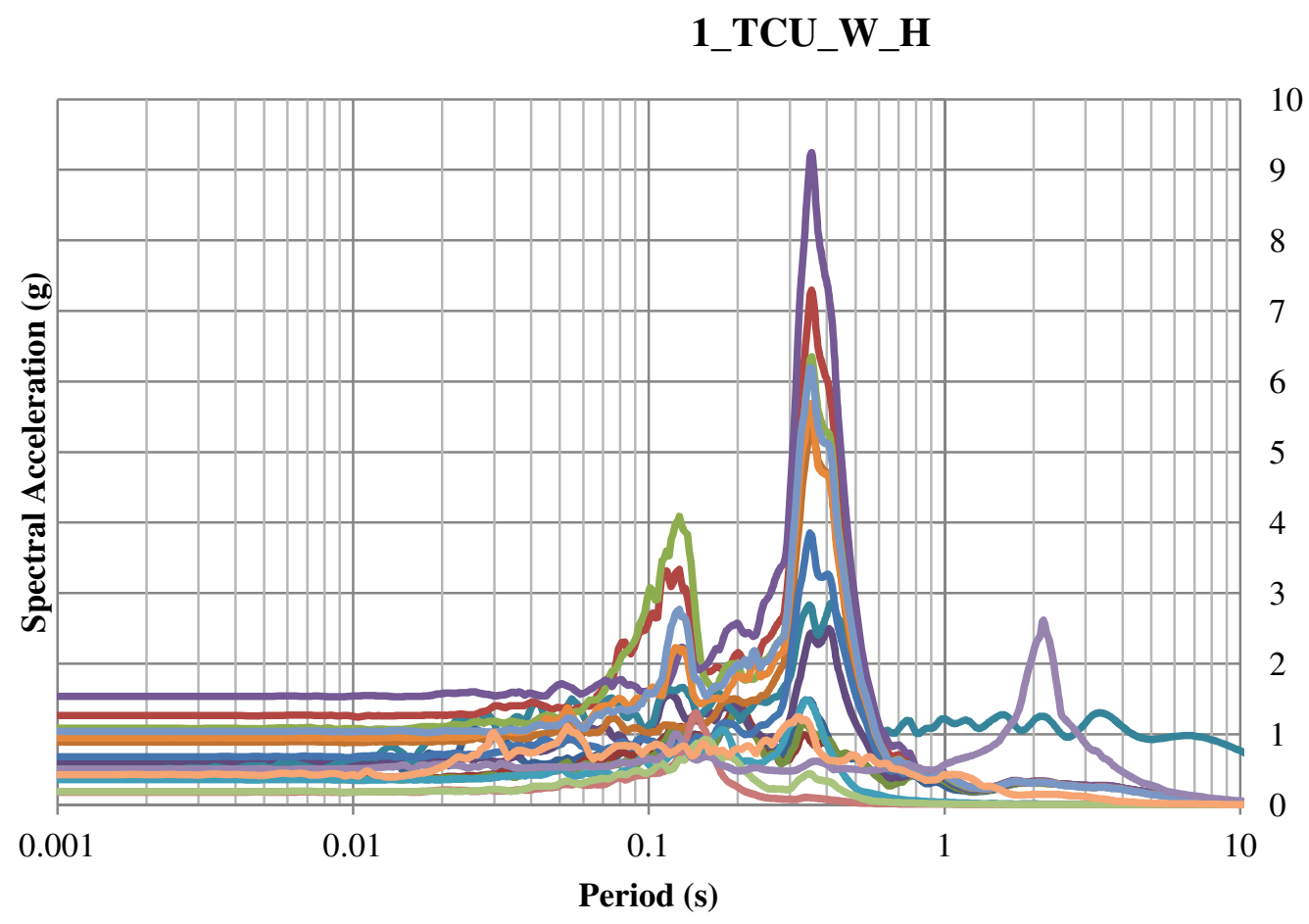

Table

$10 \longrightarrow 1-\mathrm{F}$

$9 \longrightarrow 1-\mathrm{M}$

$8 \longrightarrow 2-\mathrm{M}$

7 - $3-\mathrm{F}$

$6 \longrightarrow 3-\mathrm{M}$

$5 \begin{array}{r}5-\mathrm{F} \\ -5-\mathrm{C}\end{array}$

$4 \longrightarrow 5-\mathrm{O}$

$3 \longrightarrow 5-\mathrm{P}$

Hor_north
Hor_south

$\longrightarrow$ Ver_W

$\longrightarrow$ Ver_E

Weight

$\longrightarrow$ Table

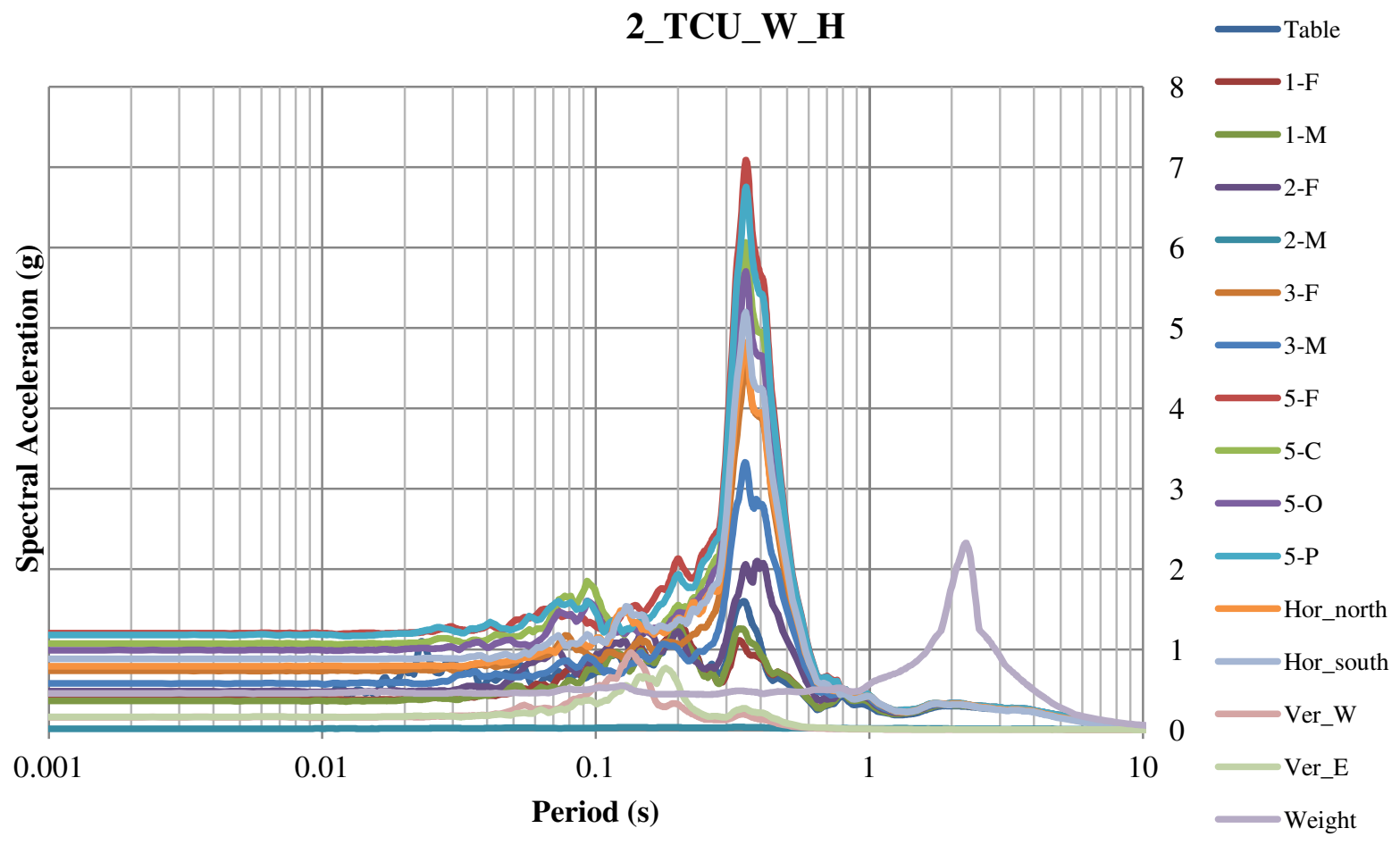



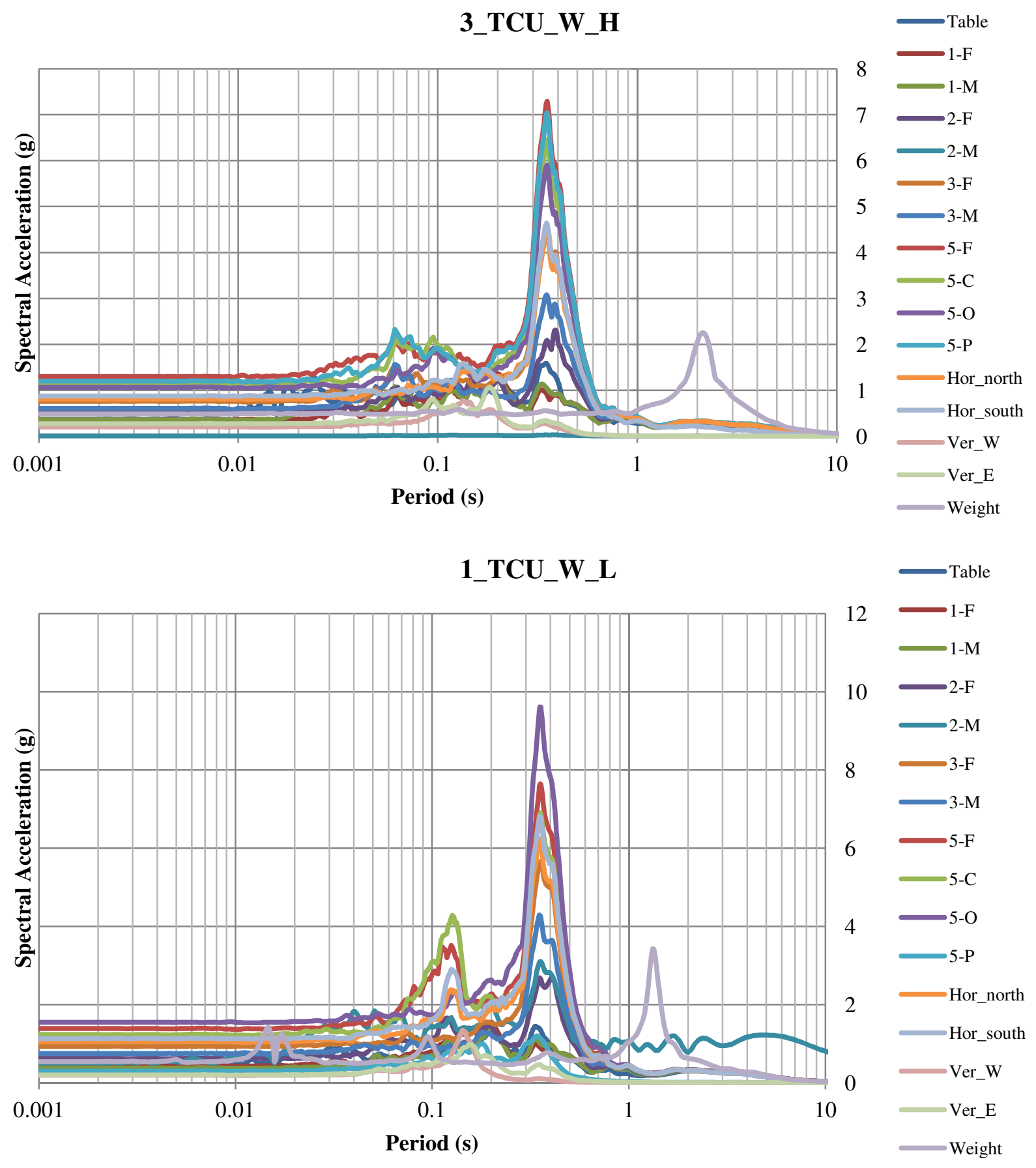

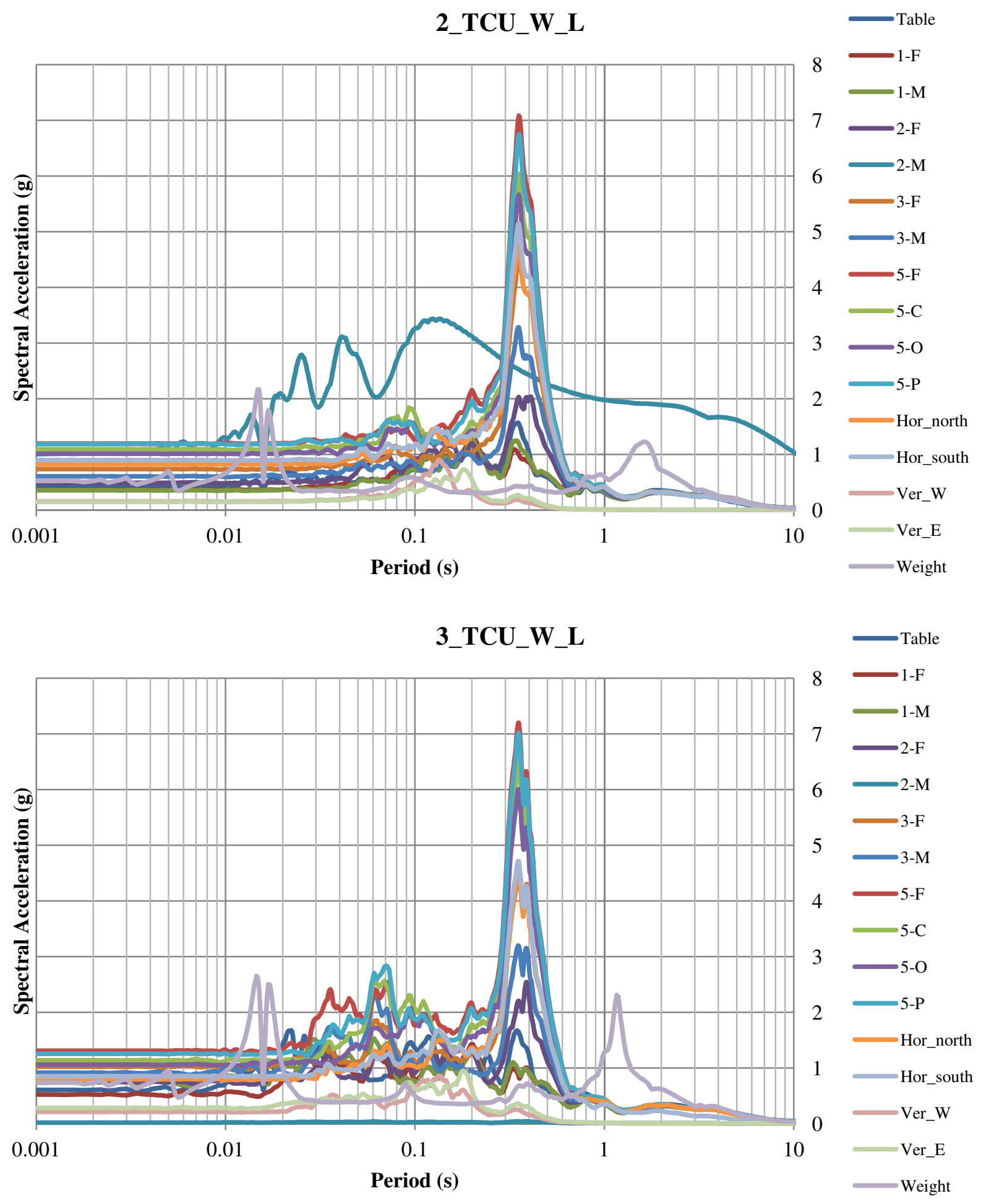

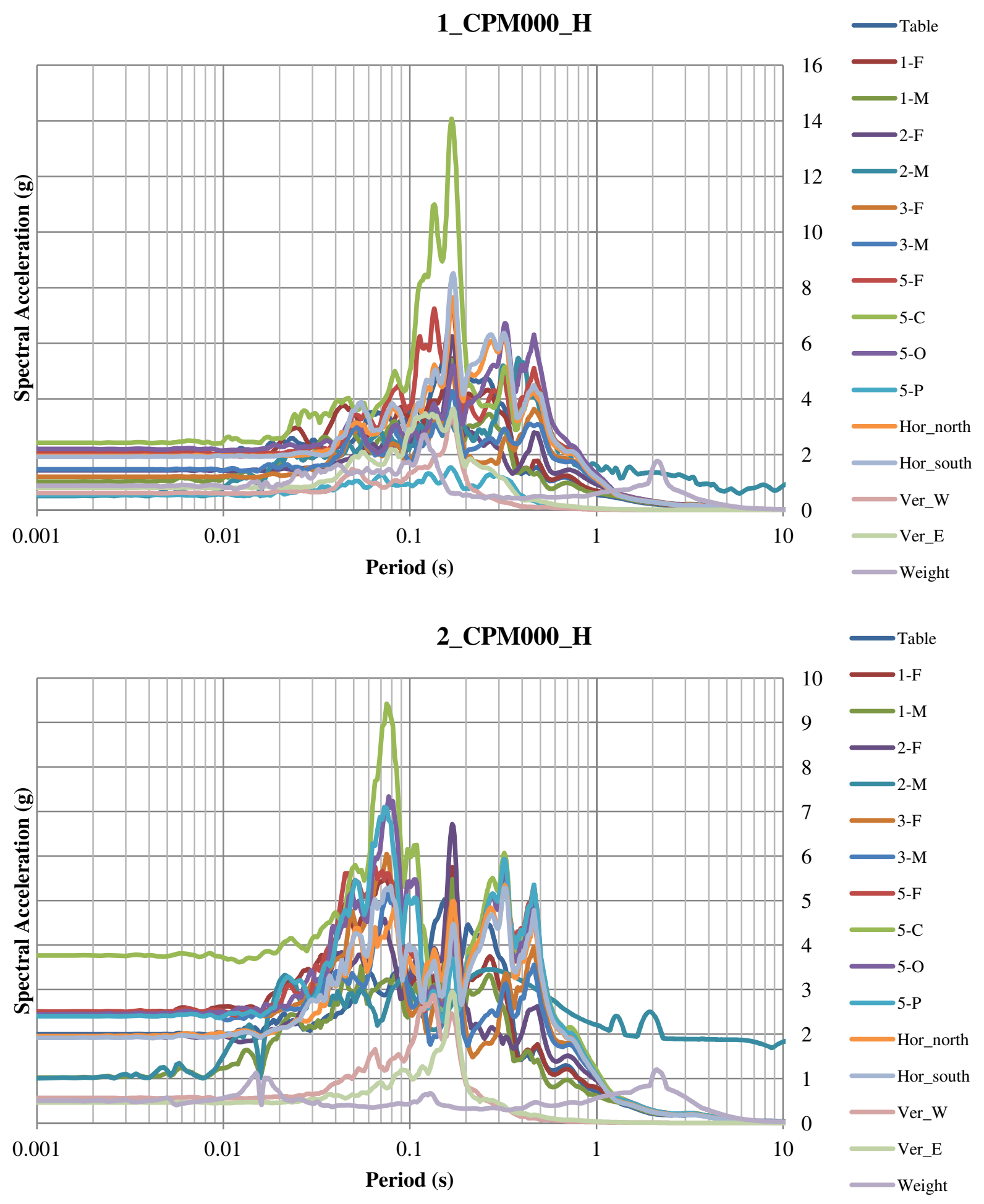

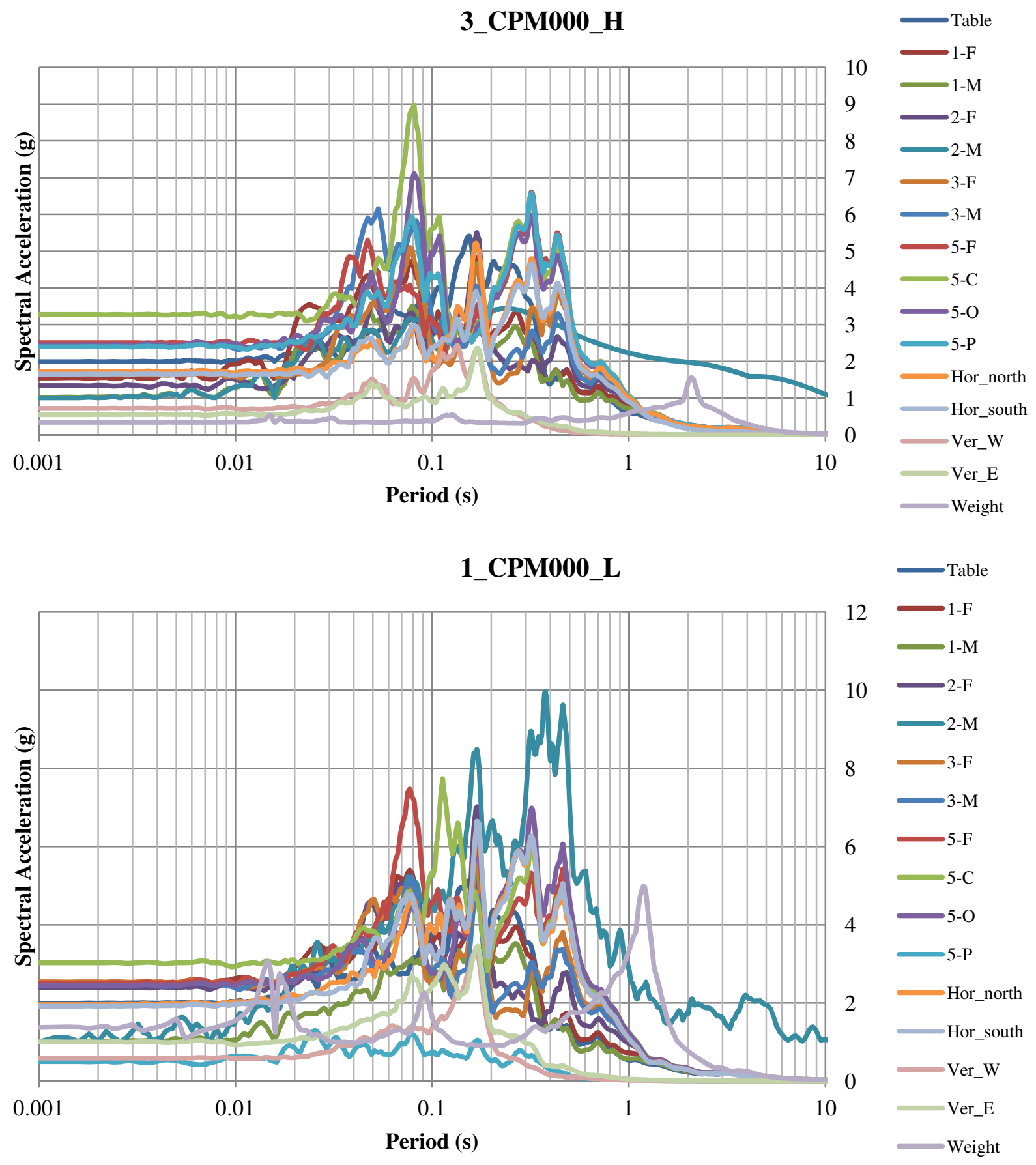

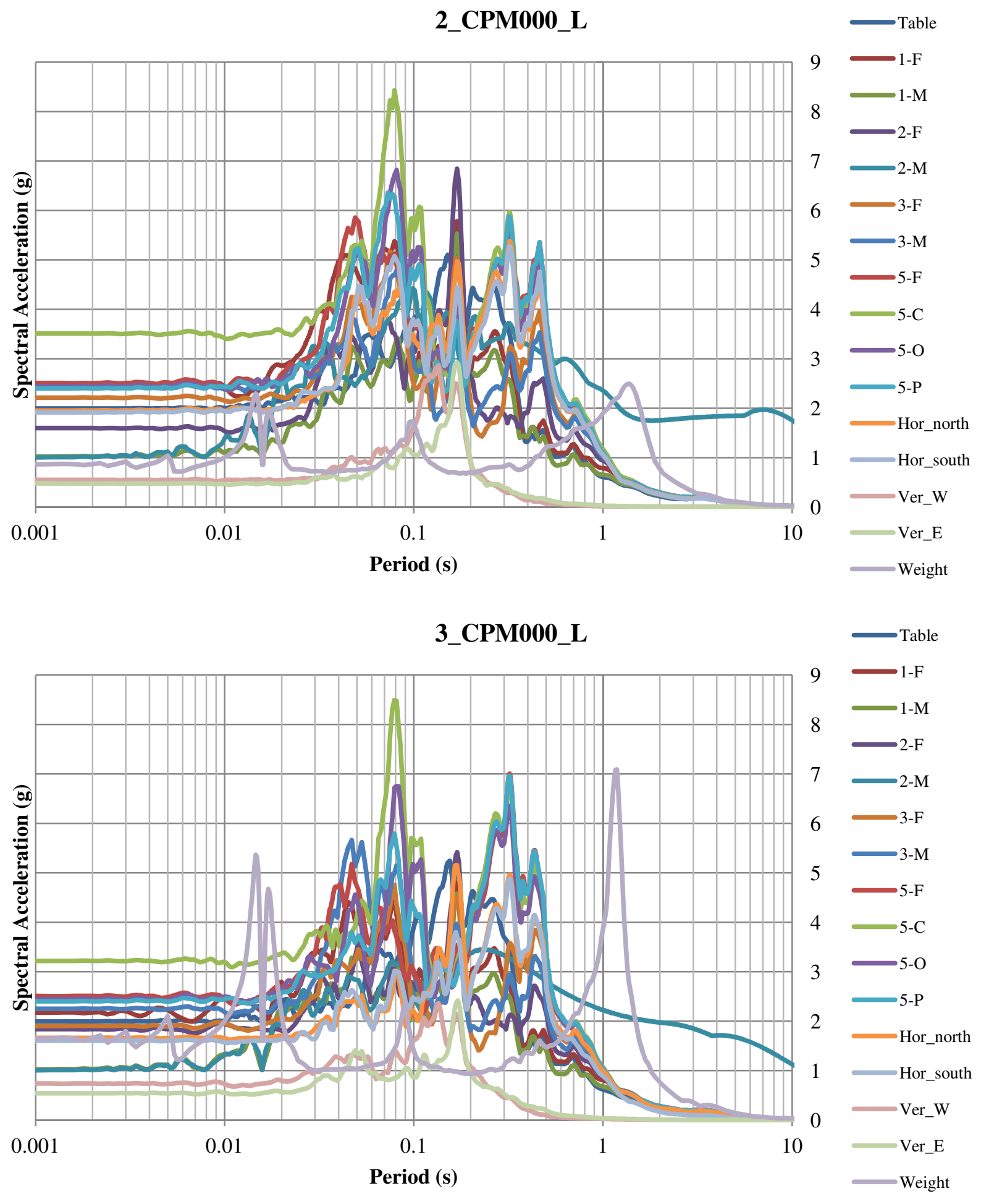

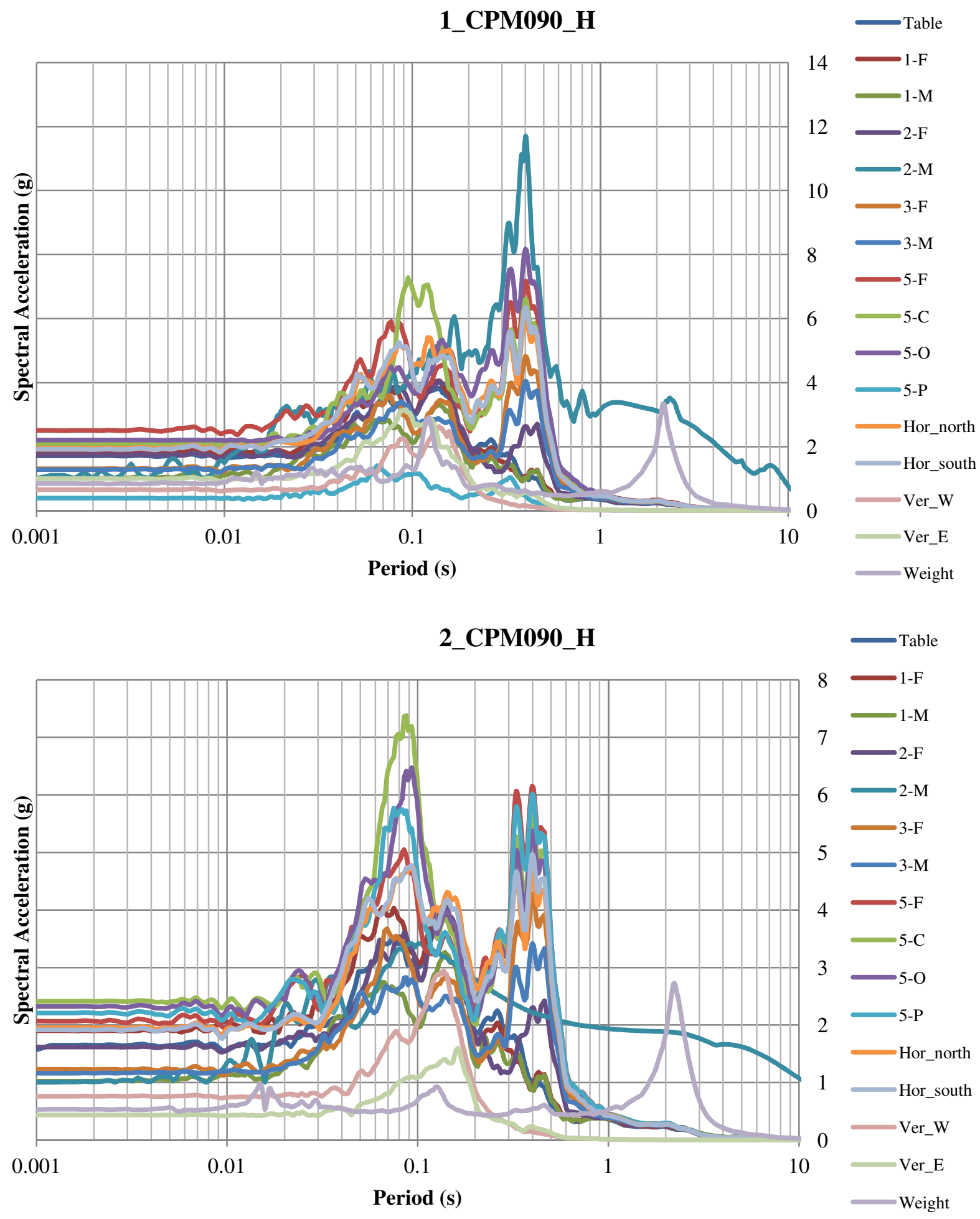

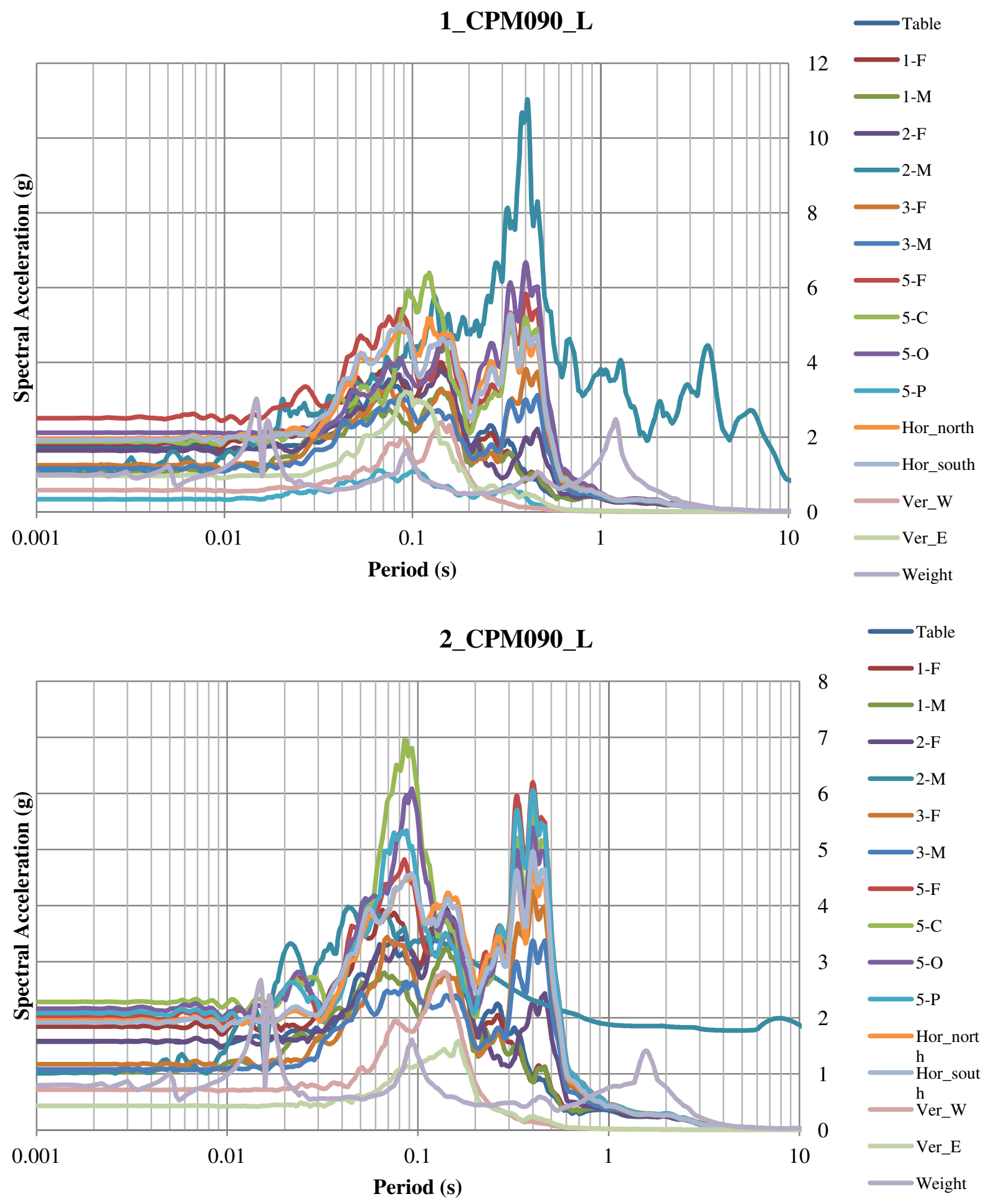


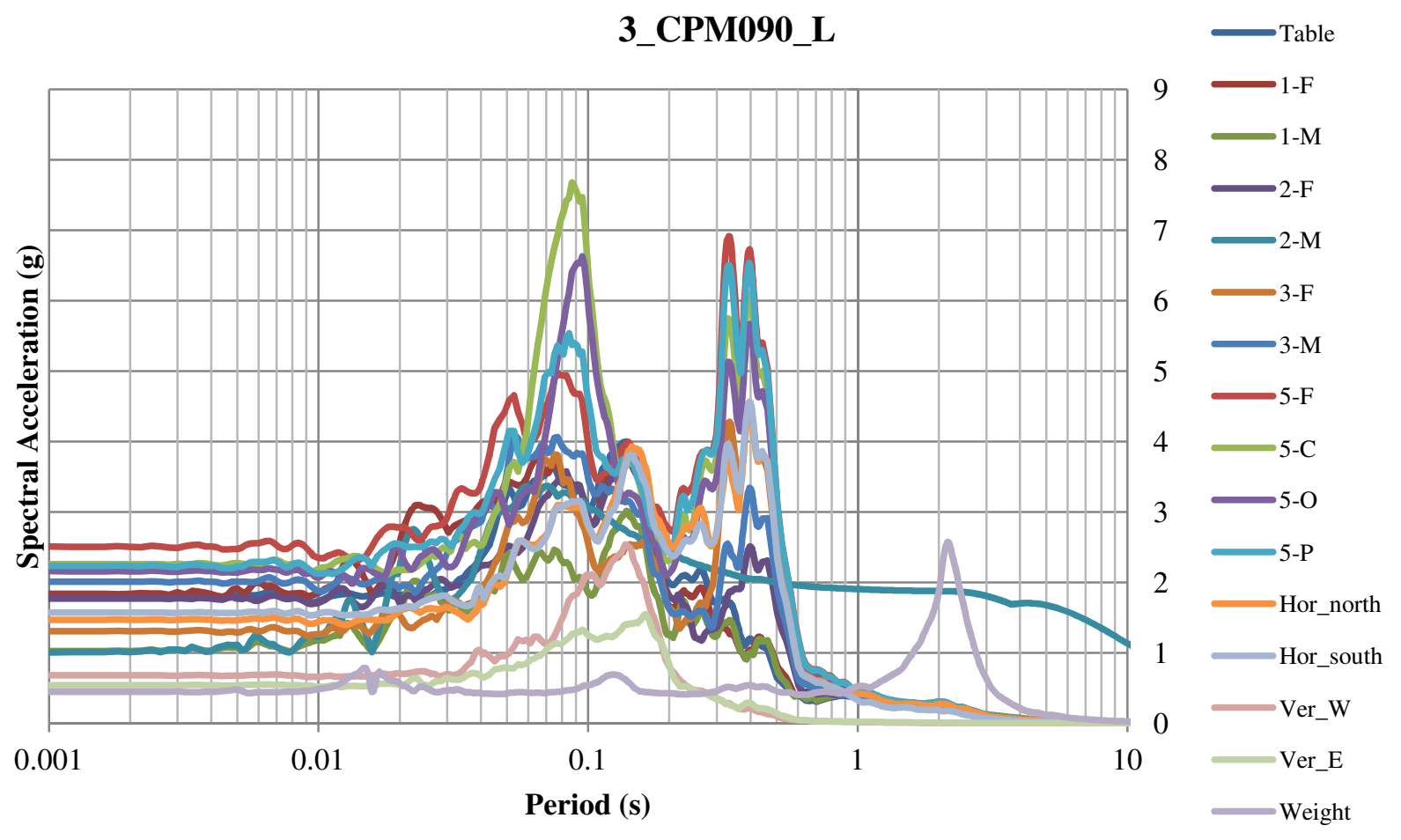



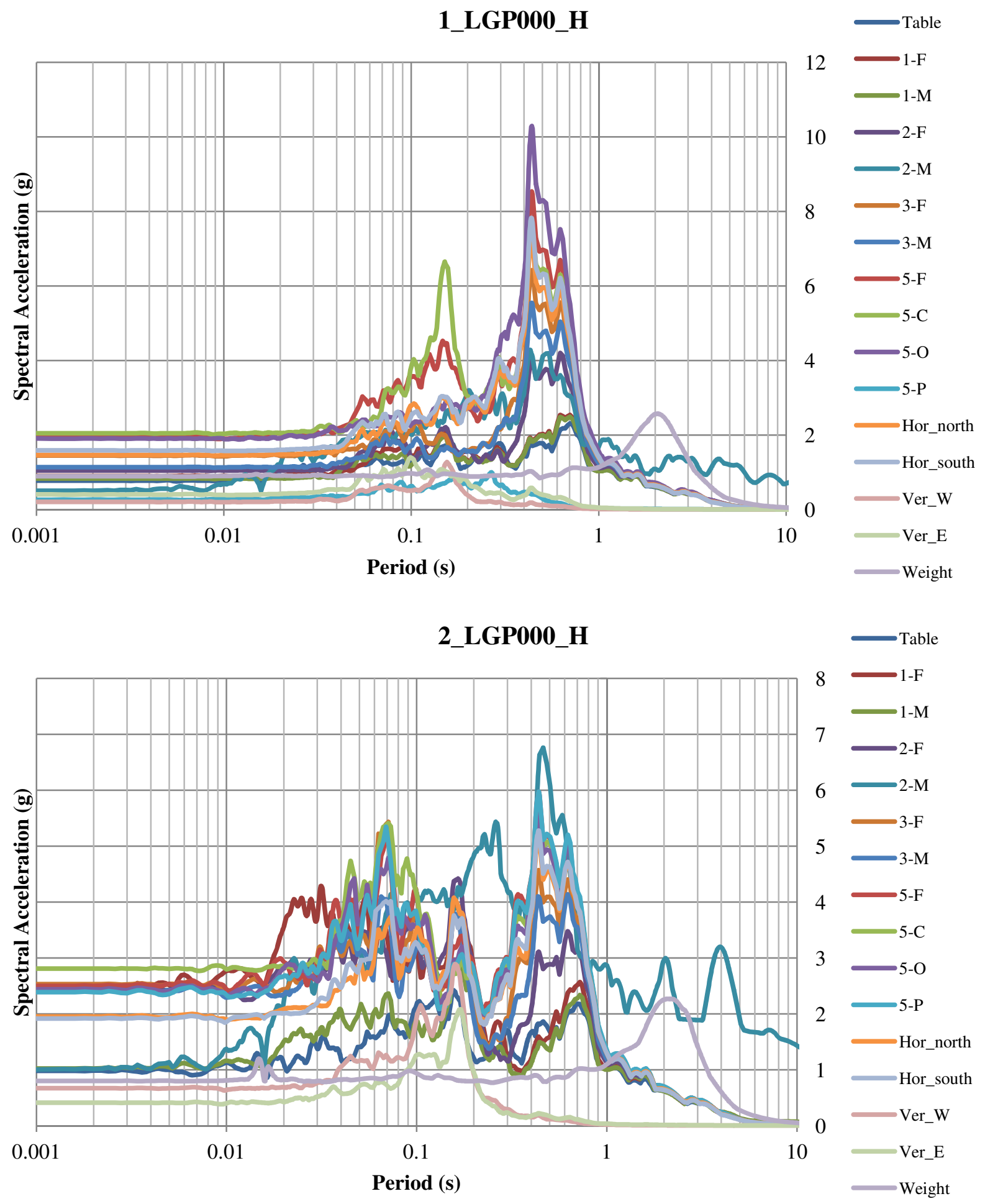

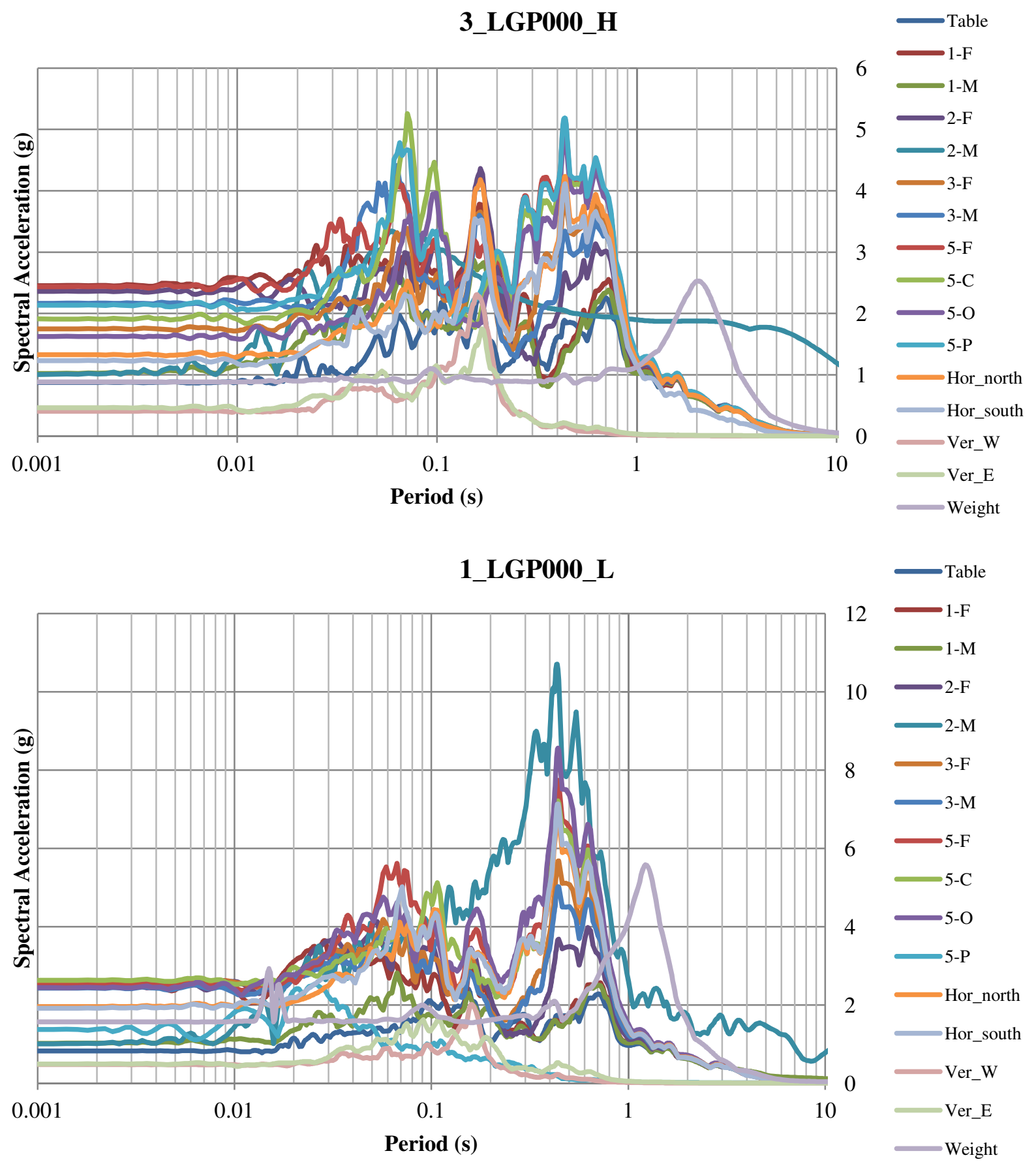

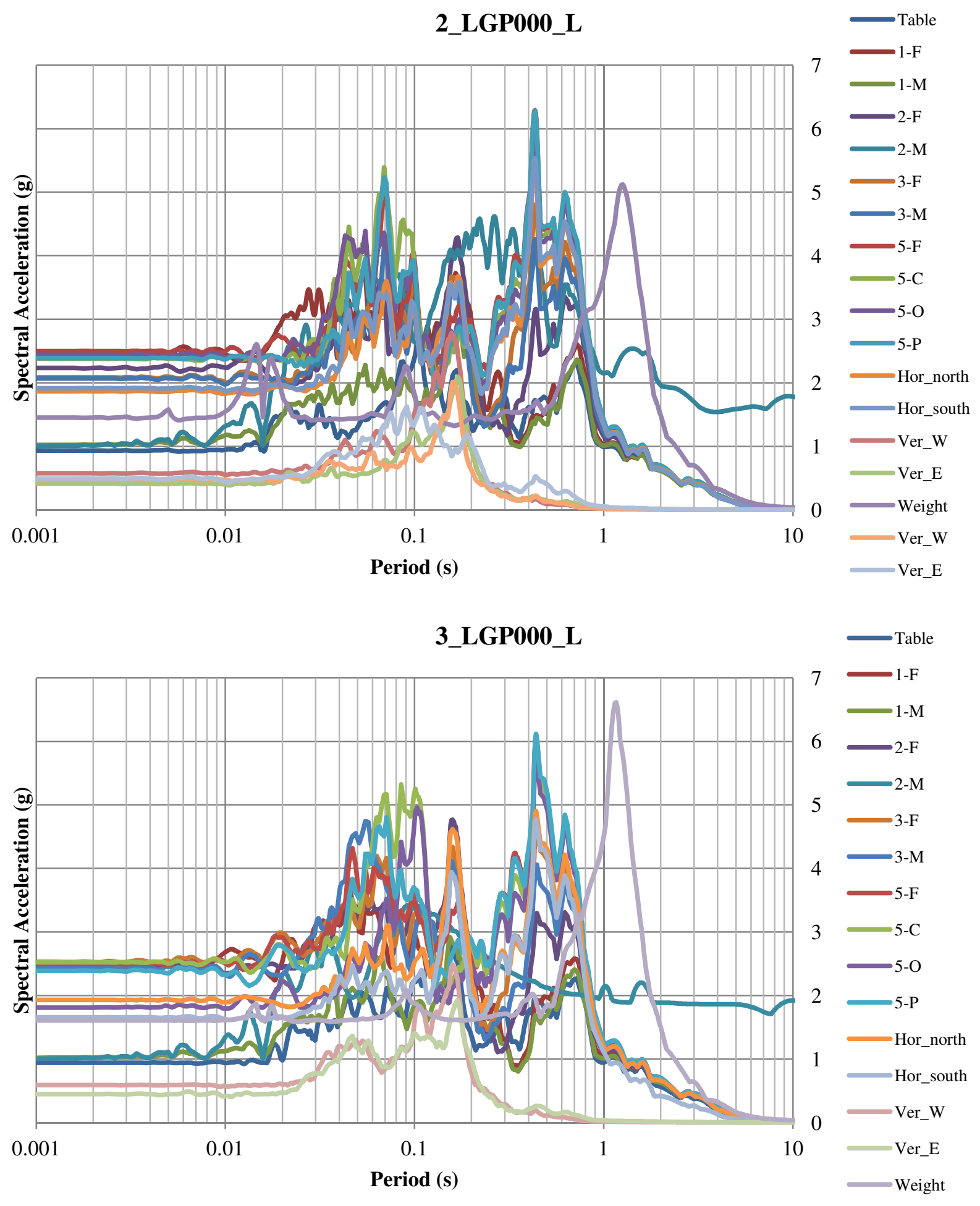

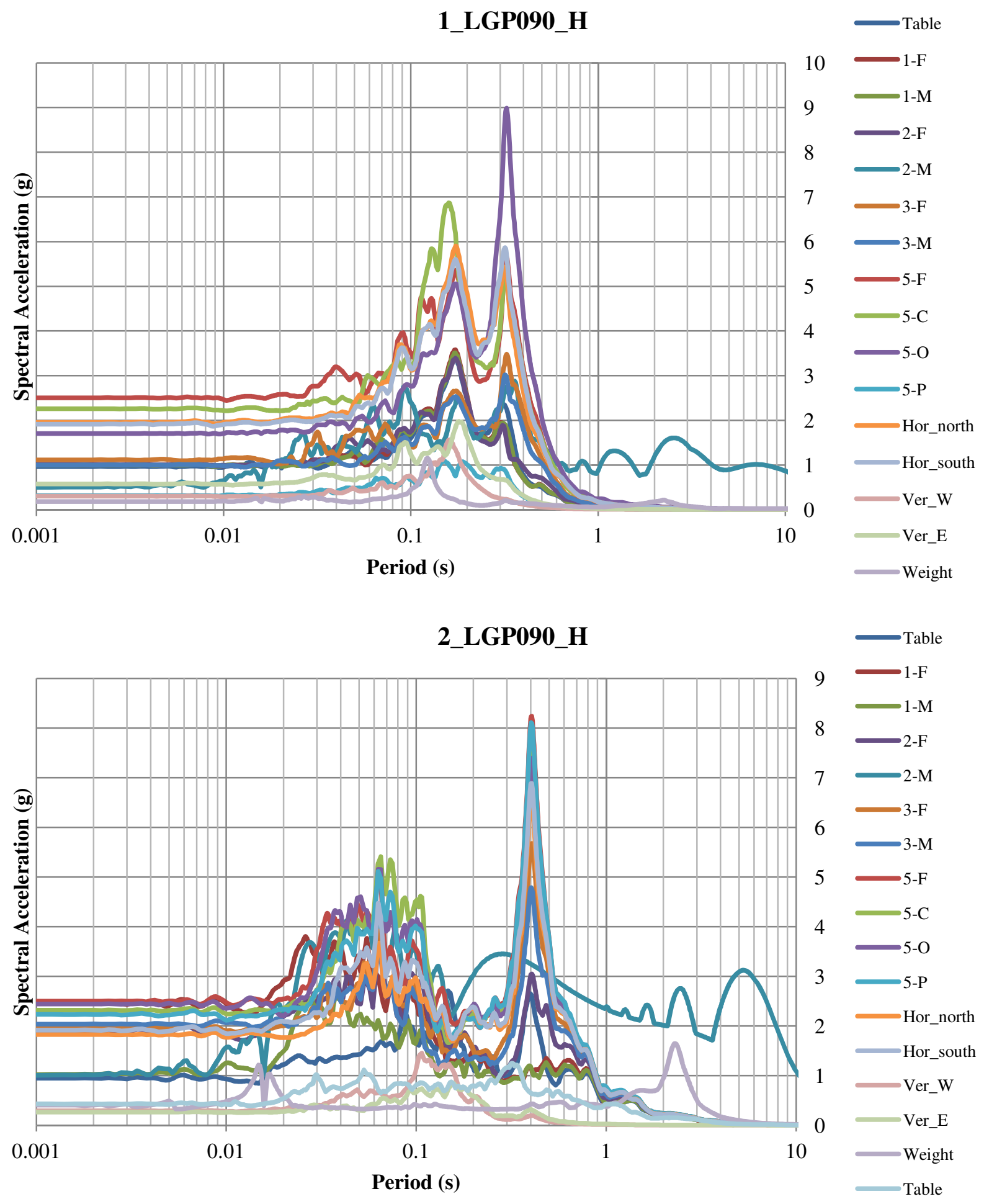

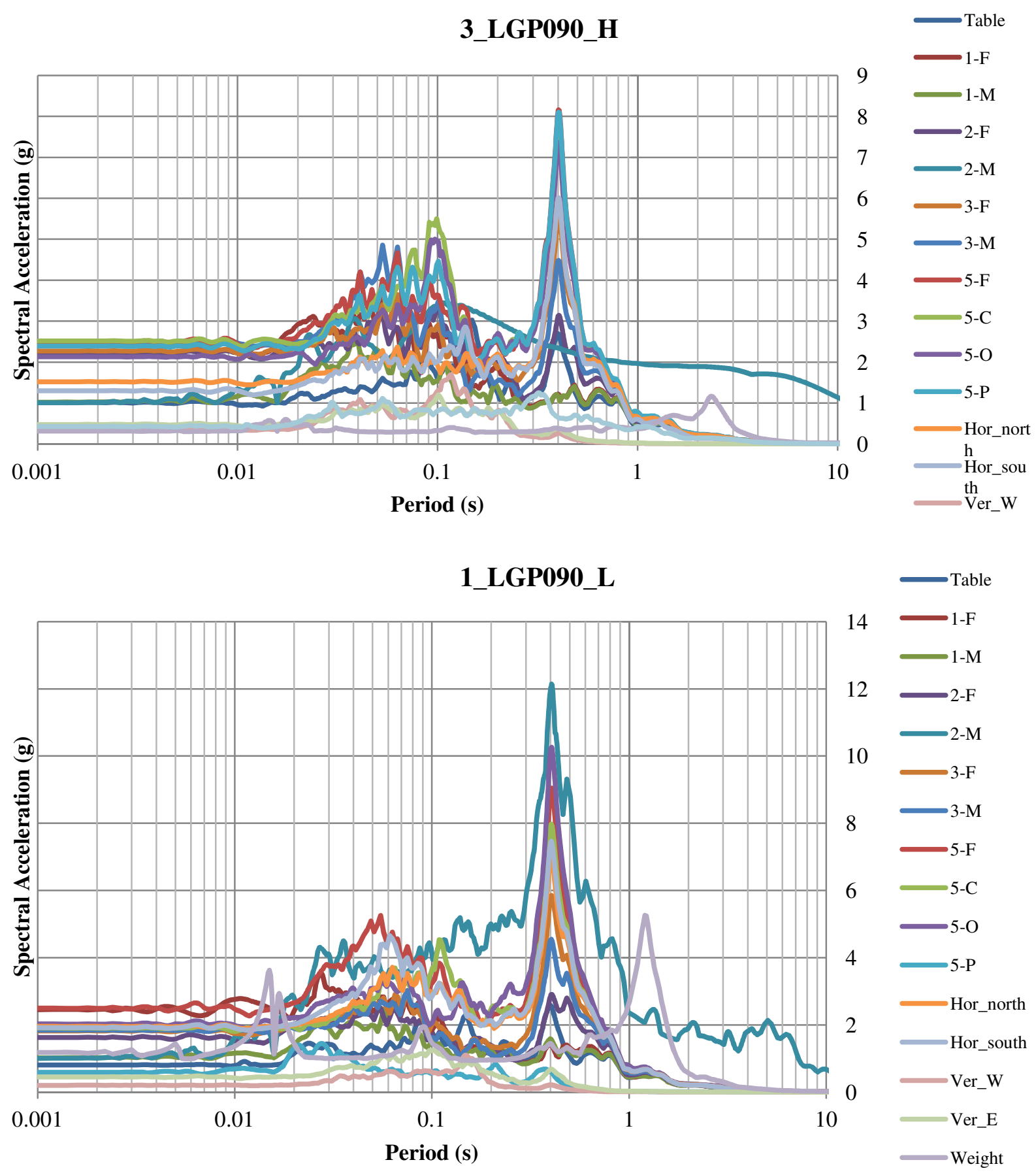

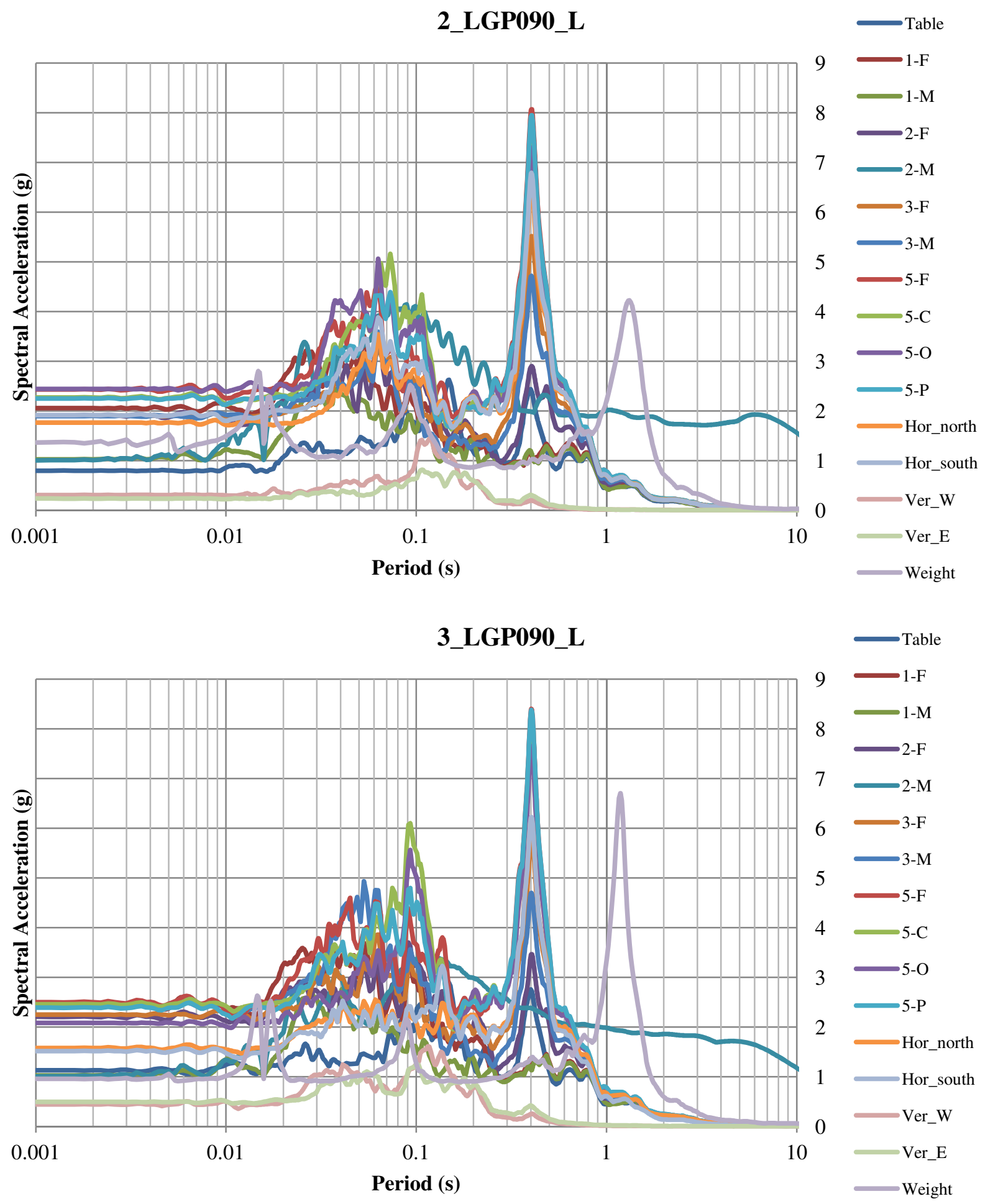

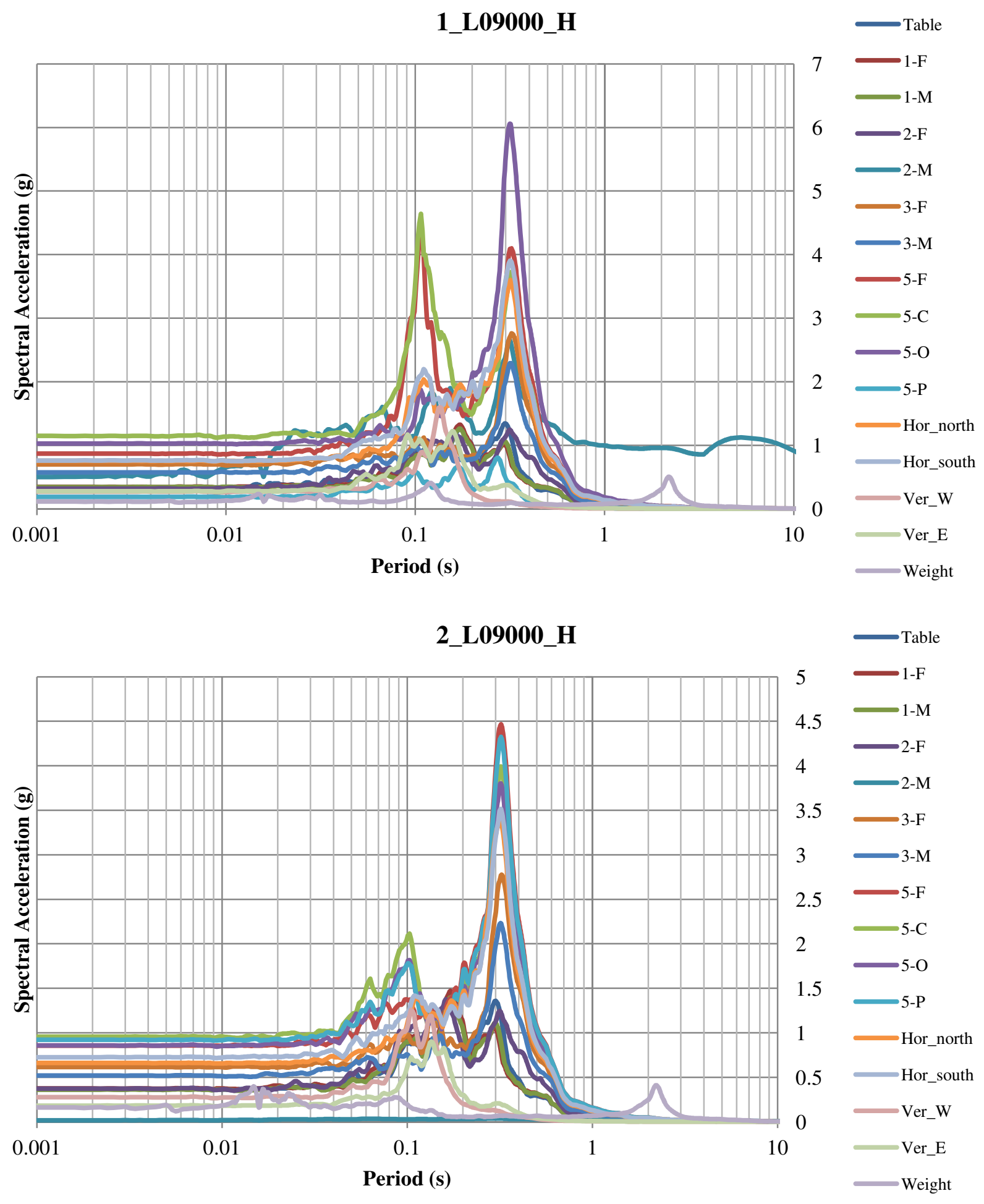

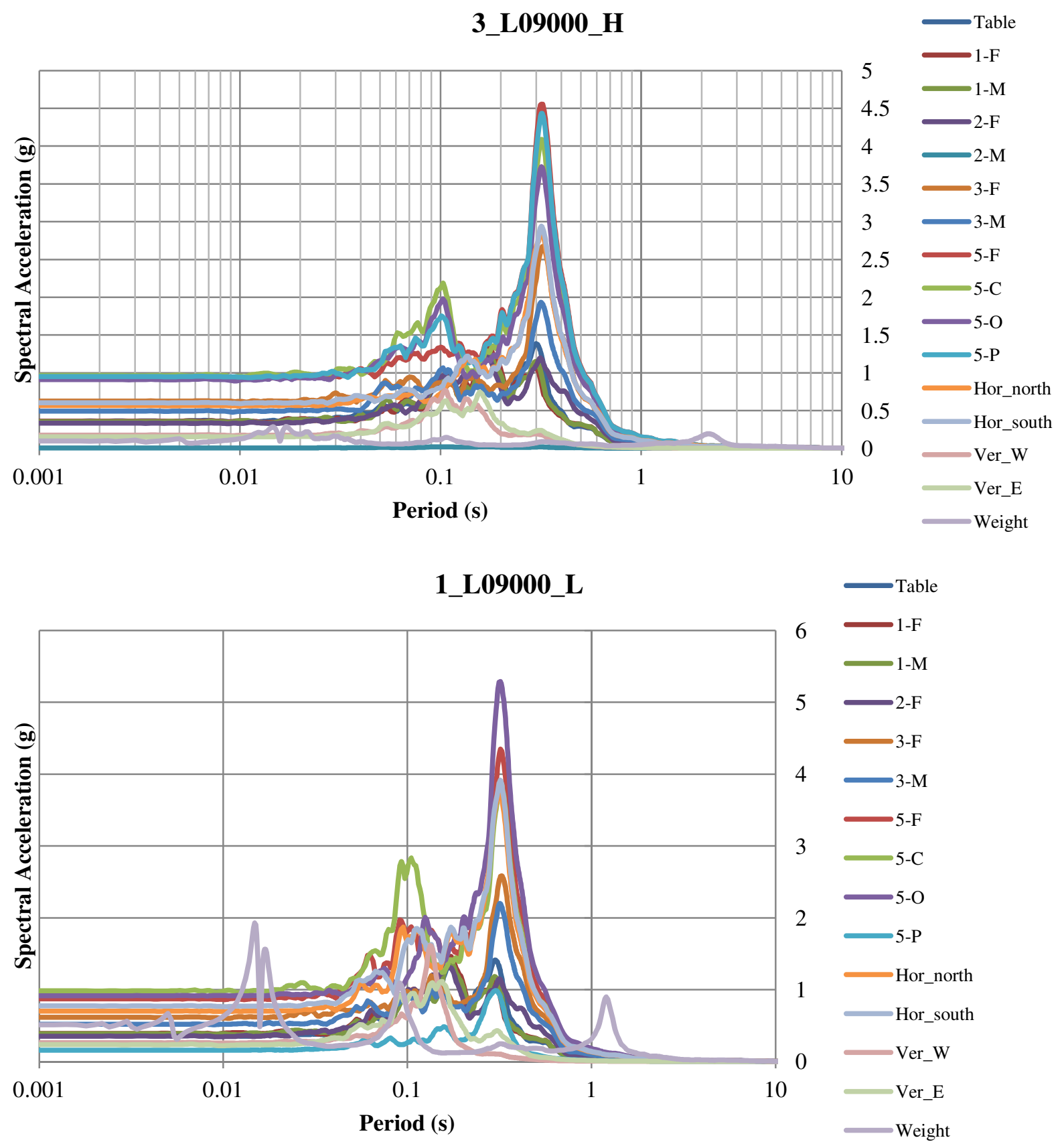
2_L09000_L
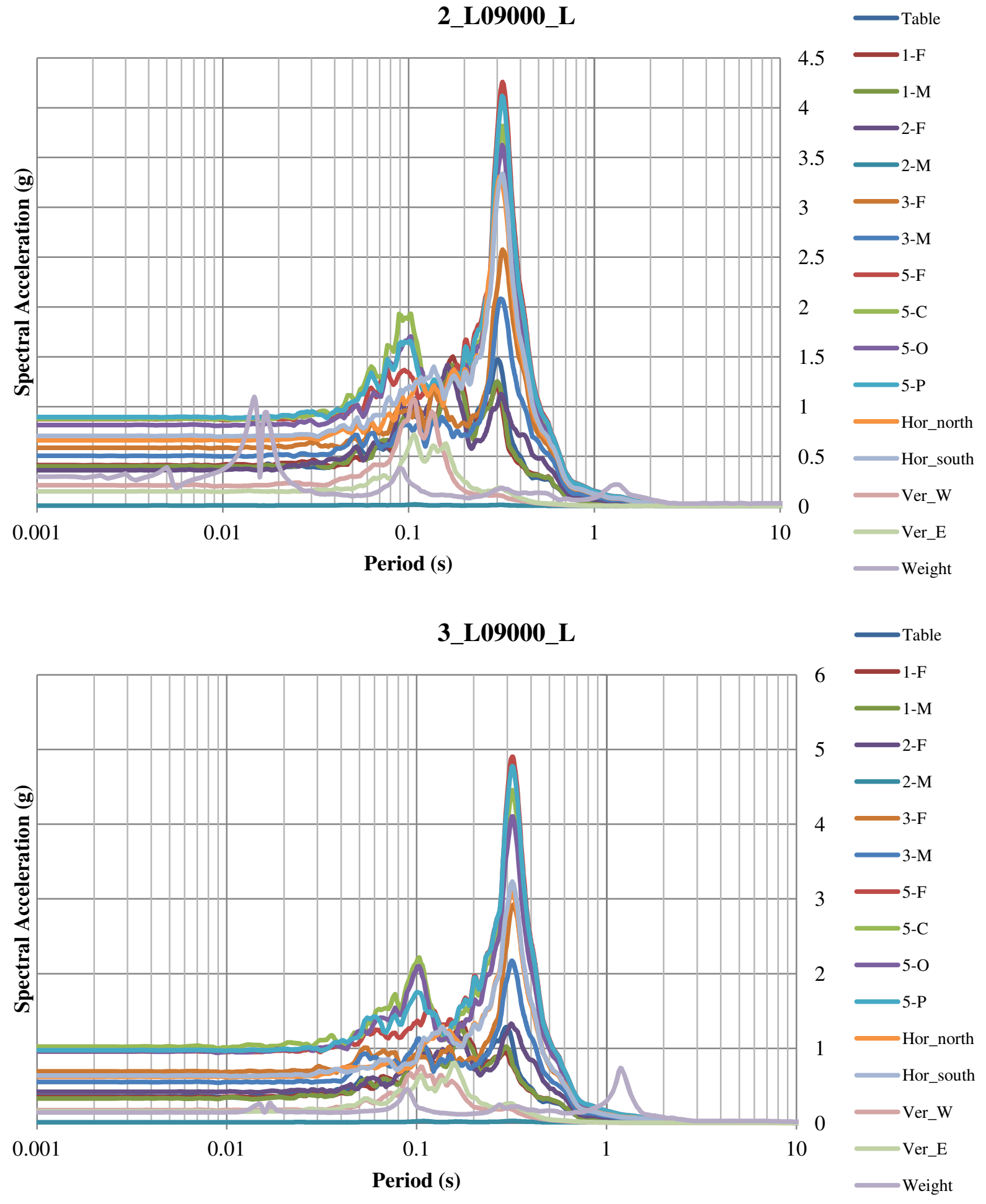

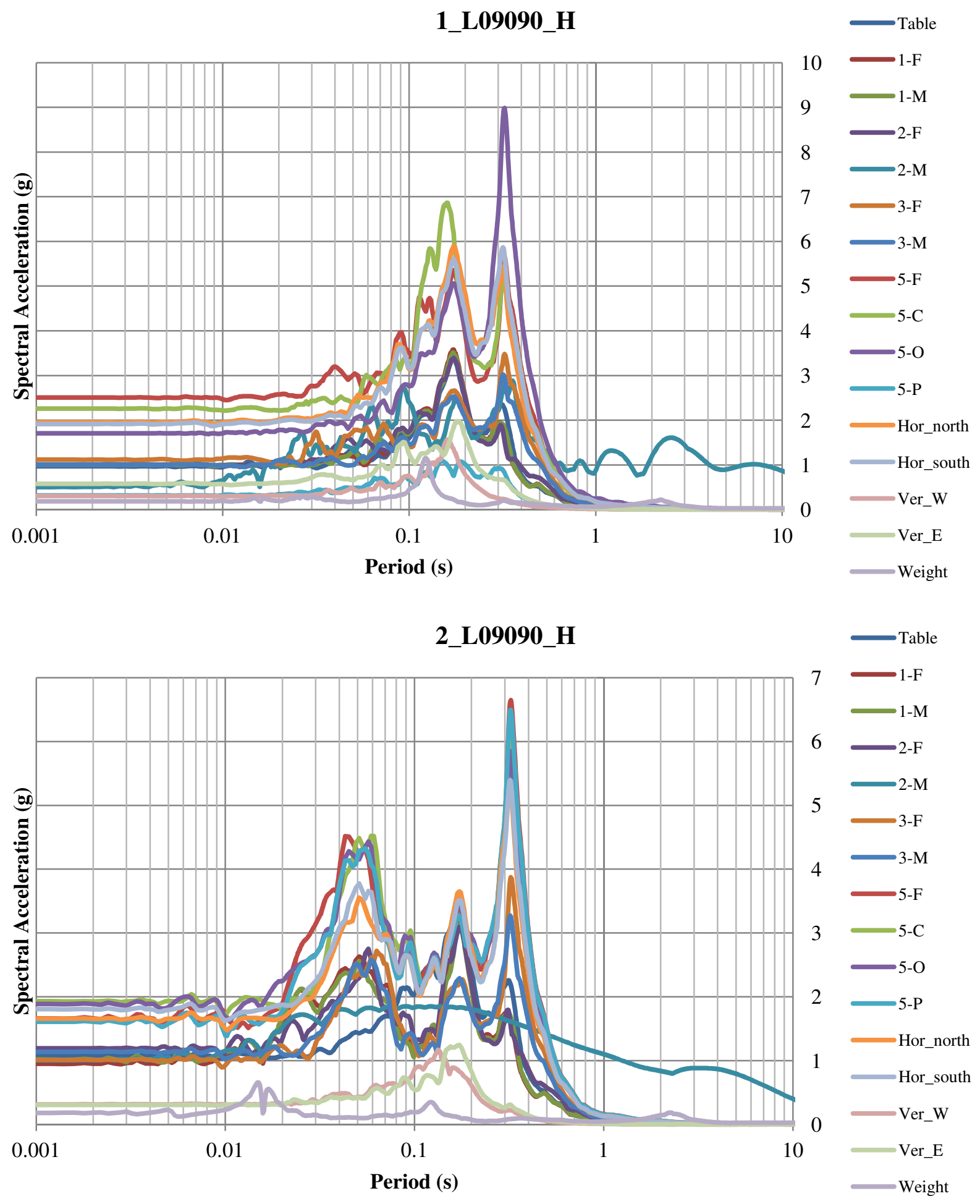

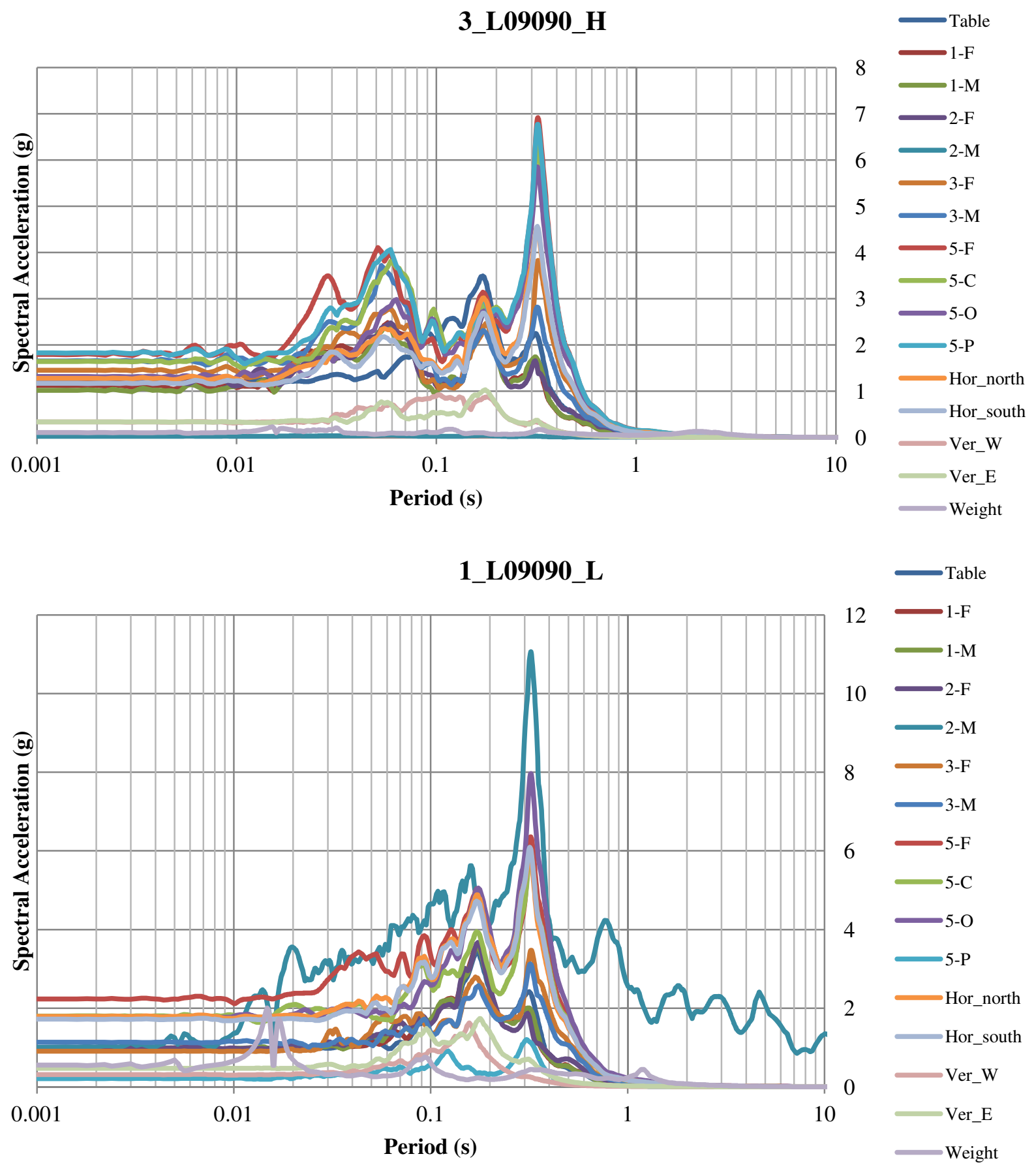


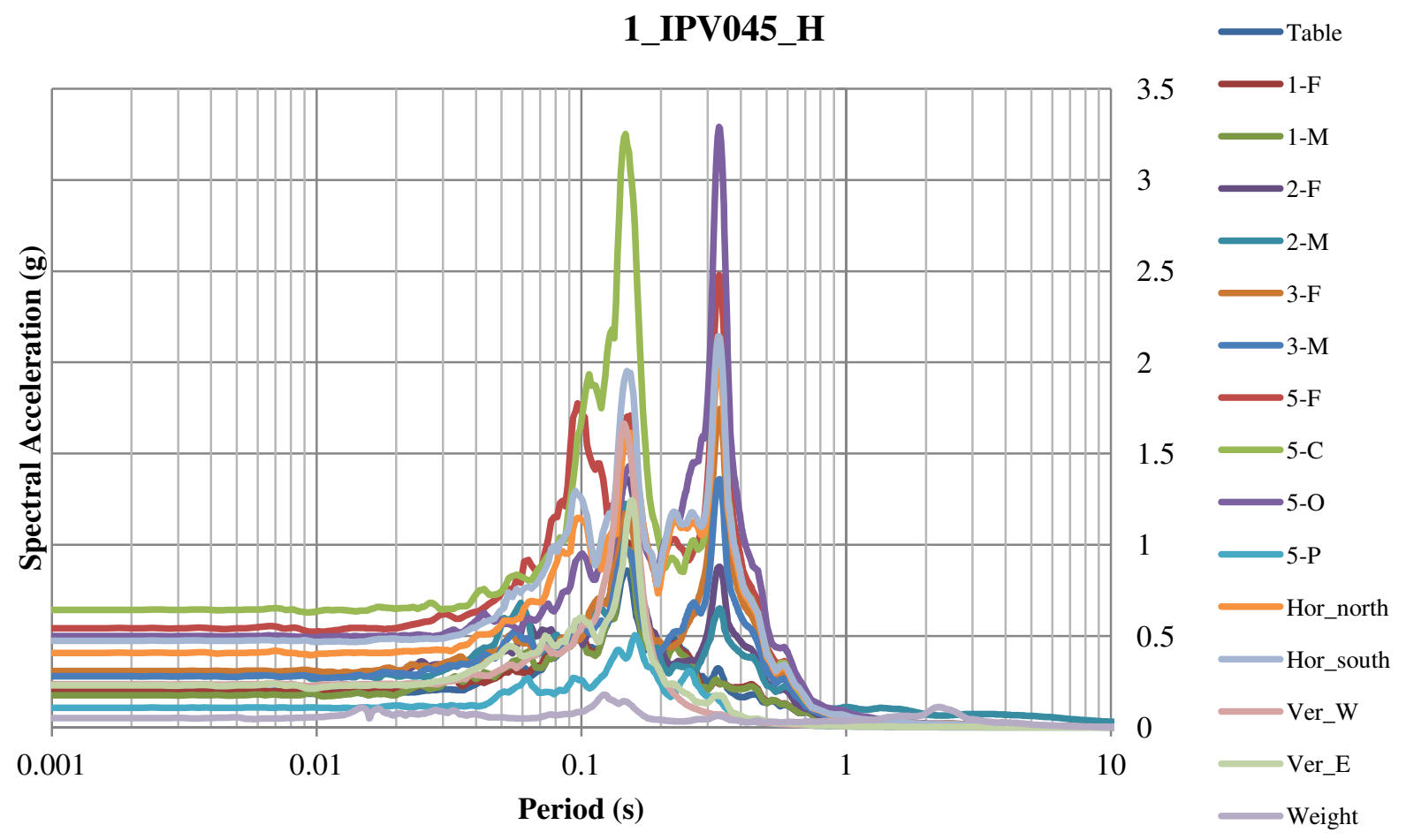




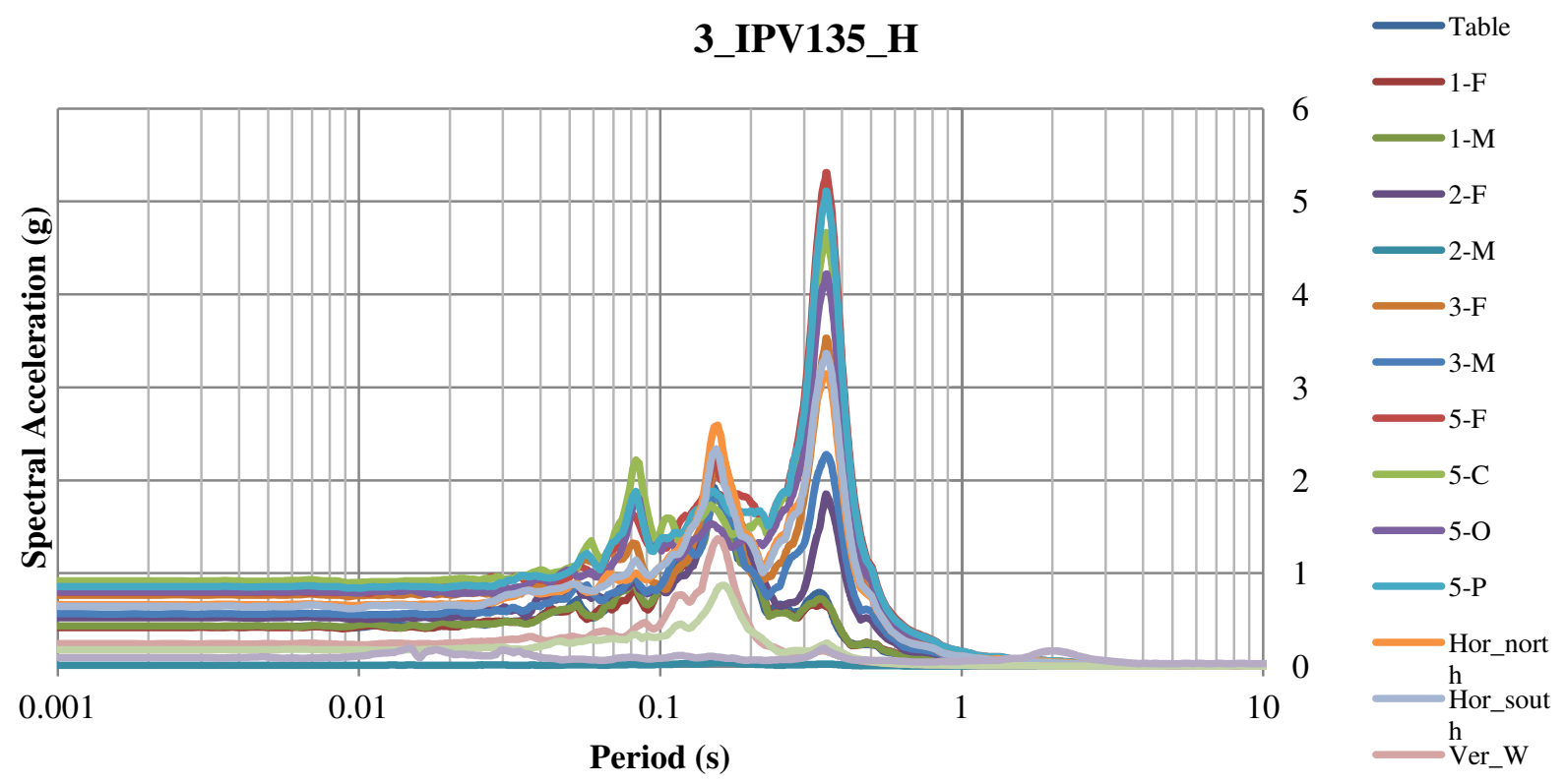




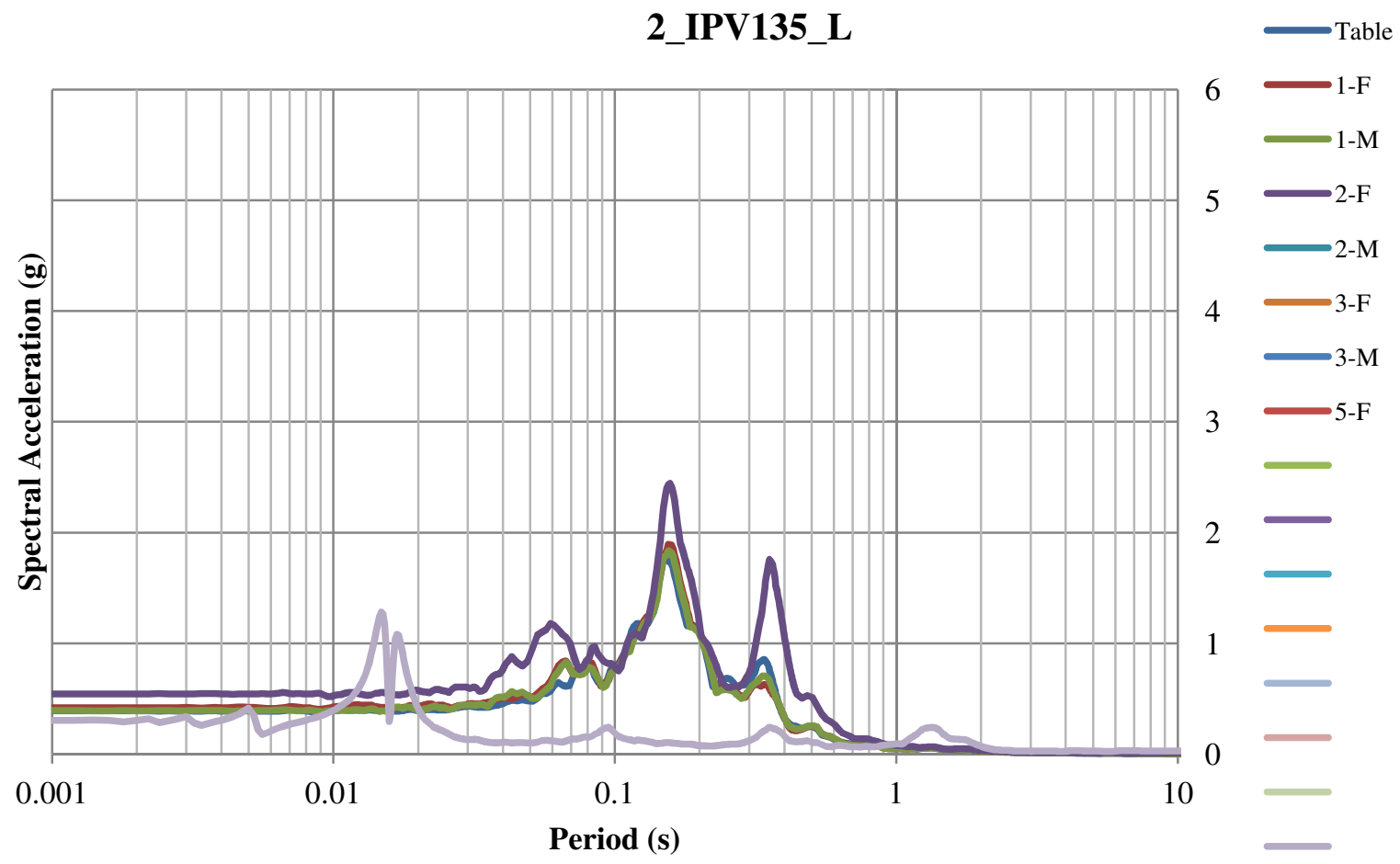

